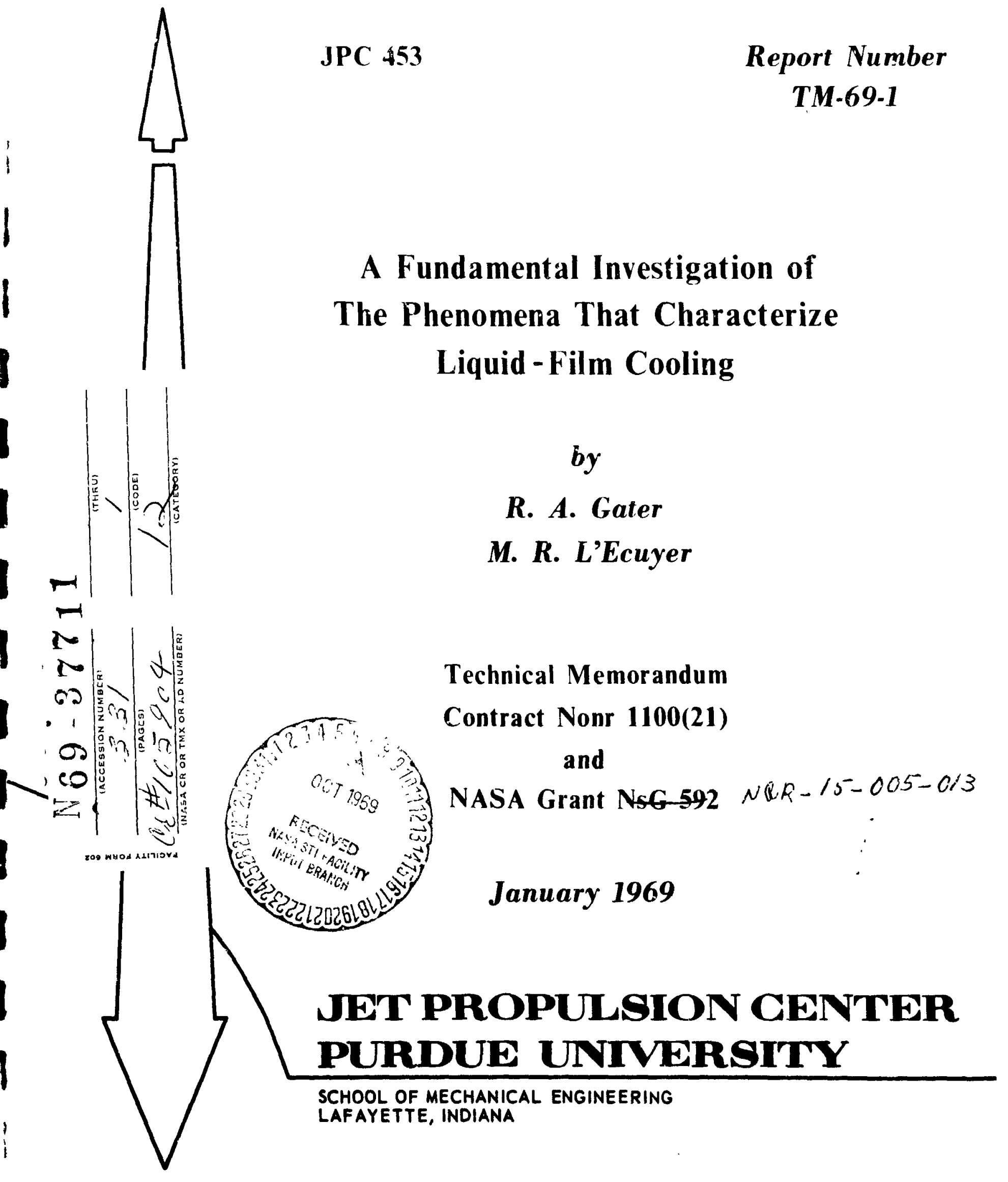




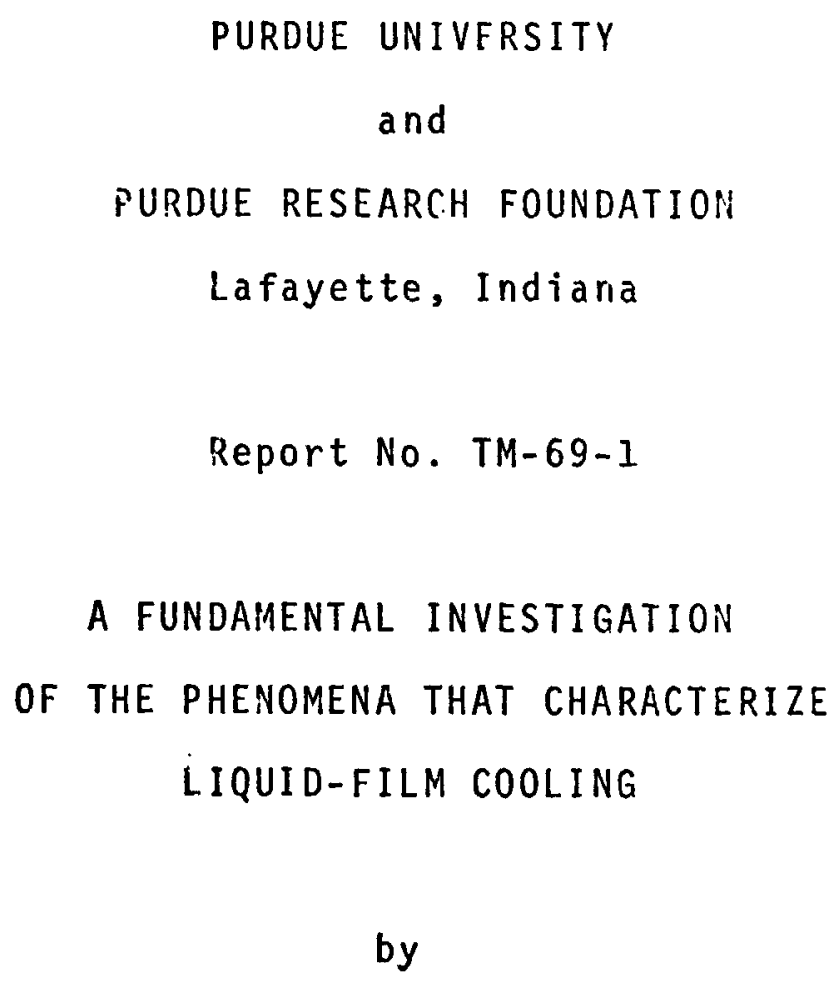




\section{i i i}

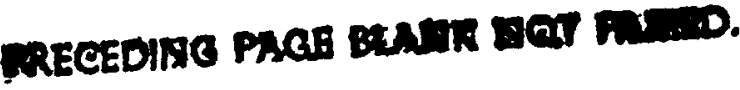

\section{ACKNOWLEDGMENTS}

The investigation reported herein was initiated under the sponsorship of the Office of Naval Research, Power Branch, under Contract Nonr $1100(21)$. The intermediate phases of the investigation were sponsored by the National Aeronautics and Space Administration under Grant NsG 592.

The authors wish to express their appreciation for the support provided by the aforementioned agencies.

The authors are also indebted to Dr. B. A. Reese, Director, Jet Propulsion Center, Purdue University, for his unwavering support of the research program and for his many helpful suggestions.

The help of Messrs. W. Timmons, G. Hurst, C. Merkel, T. Miller and T. Virgin in the fabrication of the research apparatus is gratefully acknowledged.

The authors also wish to thank Mrs. Joyce Gater for her typing and repeated retyping of the original manuscript, and Mrs. Virginia Hawks for her expert typing and invaluabie assistance in the preparation of the final manuscript.

Finally, the aucuurs wish to express their appreciation to Dr. R. B. Gaither, Chairman, Department of Mechanical Engineering, University of Florida, for his cooperation and assistance in the publication of this report. 
Page

LIST OF TABLES . . . . . . . . . . . . . . . . . . ix

LIST OF FIGURES . . . . . . . . . . . . . . $x$

ABSTRACT . . . . . . . . . . . . . . . . . xvi

1. INTRODUCTION . . . . . . . . . . . . . . 1

1.1 General Discussion . . . . . . . . . . . 1

1.2 A Statement of the Problem . . . . . . . 2

1.2.I The Liquid-Film Cooled Region . . . . 4

1.2.2 The Gas-Vapor Cooled Region . . . . . 6

1.3 A General Review of the Literature . . . . 8

1.4 The Scope of the Investigation . . . . . . . 15

2. THE EXPERIMENTAL INVESTIGATION . . . . . . . . . 18

2.1 The Basic Experimental Apparatus . . . . . 18

2.2 The Experimental Model. . . . . . . . . 25

2.3 The Experimental Parameters......... 30

2.4 The Photographic Data for the Film Surface
Characteristics................. 33

2.4.1 The Influence of the Liquid Parameters

on the Film Surface Characteristics

2.4.2 The Influence of the Gas Stream

Parameters on the film Surface

2.4.3 Summary

2.5 The Data for Mass Transfer for a Constant

Velocity Gas Stream............ 
Page

2.5.1 The Influence of the Surface Tension and the Liquid Viscosity on the Data for Mass Transfer. ... . . 57

2.5.2 The Influence of the Gas Stream Parameters on the Data for Mass

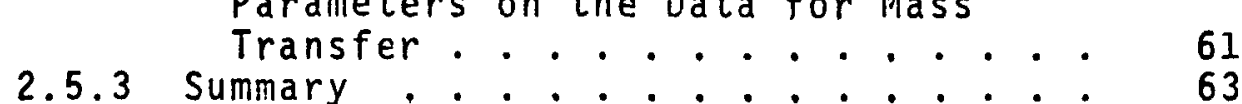

2.6 The Data for Mass Transfer for an Accelerating Gas Stream........ . 64

2.7 The Data for the Maximum Liquid Film

Temperature............... 67

3. CORRELATION OF THE EXPERIMENTAL DATA . . . . . . 71

3.1 Correlation of the Data for Mass Transfer for a Constant Velocity Gas Stream ..... 72

3.1.1 General Discussion......... 72

3.1.2 The Evaluation of the Rate of Simple Mass Transfer......... 79

3.1.3 The Correlation of the Entrainment

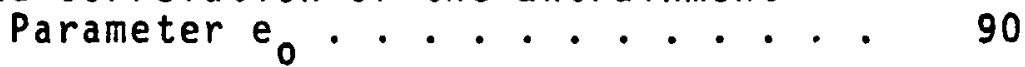

3.1.4 The Physical Significance of the Correlation for $e_{0} . . .+. . .998$

3.1.5 The Correlation of the Roughness

3.1.6 The Comparison of the Predicted and the Measured Rates of Mass Transfer 108

3.2 The Modification of the Basic Correlation for Mass Transfer to Account for an Arbitrary Film Cooled Length . . . . . 117

3.2.1 Model and Analysis......... 118

3.2.2 An Empirical Approximation to the
Analytical Result ......... 124

3.3 Correlation of the Data for Mass Transfer for an Accelerating Gas Stream . . . . . . 129

3.3.I The Evaluation of the Simple Rate of Mass Transfor for an Accelerating Gas Flow.......... 129

3.3.2 The Model and Analysis....... 133

3.3.3 The Comparison of the Predicted and the Measured Rates of Mass Transfer 
3.4 Correlation of the Experimental Data for the Maximum Liquid Temperature... . .

3.4.1 A Method for Predicting the Maximum

3.4.2 Comparison of the Experimental and the Predicted Results for the Maximum Film Temperature . . . . .

4. CORRELATION OF THE DATA OF THE PRIMARY

4.1 Introduction .............. 150

4.2 An Approximate Method for Evaluating the Surface Tension .............

4.3 The Data Due to Kinney, Abramson, and Sloop..................... 160

4.3.1 Introductory Remarks ........ 160

4.3.2 The Basic Apparatus .... . . . . 160

4.3.3 The Assumptions. 162

4.3.4 Comparison of the Present Theory With the Experimental Data.......

4.3.5 A Comment on the Presentation of Data for Liquid-Film Cooling in Terms of Dimensionless parameters

4.4 The Data Due to Emmons........... 176

4.4.1 Introductory Remarks . . . . . . 176

4.4.2 The Basic Apparatus........ . 177

4.4.3 The Assumptions .......... 177

4.4.4 Comparison of the Present Theory With

4.4.5 An Additional Comment on the Research

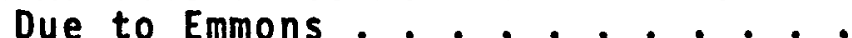

4.5 The Data Due to Hermann, Leitinger, and

Melnik.............. 189

4.5.1 Introductory Remarks : : : : 189

4.5.2 The Basic Apparatus.:. : : : : 191

4.5.3 The Assumptions .......... 193

4.5.4 Comparison of the Present Theory With the Experimental Data....... 194

4.5.5 An Illustrative Example for

$$
T_{g}^{0}=4000 R \text { and } F^{0}=500 \text { psia... }
$$


vi i

Page

5. CONCLUSIONS . . . . . . . . . . . . . . . 202

BIBLIOGRAPHY . . . . . . . . . . . . . . 206

NOTATION . . . . . . . . . . . . . . . . . . . 212

APPENDIX A-AN ANALYSIS FOR THE GAS-VAPOR COOLED

REGION....................... 217

A.l Introduction . . . . . . . . . . . 217

A.2 The Case Where the Wall is Adiabatic . . . 218

A.2.1 The Flow Model............ 218

A.2.2 The Energy Balance......... 220

A.2.3 The Evaluation of $\mathrm{m}^{\prime \prime}$. . . . . . . . 225

A.2.4 A Special Case... . . . 229

A.2.5 Experimental Data for the Adiabatic-

Hall Temperature From Reference

(19)............... 232

A.3 The Case Where the Wall is Cooled

Externally............... 234

A.4 An Observation on the Data of Emmons for
Gas-Vapor Cooling............ . . 237

APPENDIX B--DESCRIPTION OF APPARATUS . . . . . . . . 241

B.l General Description . . . . . . . . . . 241

B.2 The Gas Generator . . . . . . . . . 247

B.3 The Test Section and Test Plate....... 260

B.4 The Separator . . . . . . . . . . . 266

APPENDIX C--EXPERIMENTAL PROCEDURE . . . . . . . . . 270

C.1 Pre-Run Preparations . . . . . . . . . 270

C. 2 In-Rur Procedure . . . . . . . . . . . 272

C.3 Post-Run Procedure . . . . . . . . . . . 273

APPENDIX D--PHYSICAL PROPERTIES OF FILM COOLANTS • • • 274

D.1 Subject Investigation . . . . . . . . . . 274

D.2 Investigation Due to Emmons . . . . . . . 279 


\section{yi i}

Page

APPENDIX E--A SAMPLE CALCULATION FOR THE MAXIMUM

LIQUID TEMPERATURE . . . . . . . . . . . . . . . 283

APPENDIX Fa-DATA REDUCTION . . . . . . . . . . . 286

F.l General Data Reduction.......... 286

F.2 Gas Stream Parameters . . . . . . . . 288

F.3 Mass Transfer Data. . . . . . . . . . 291

APPENDIX G--EXPERIMENTAL DATA . . . . . . . . . 292 
Table

Page

1. Nominal Flow Parameters--Constant Velocity

Gas Stream.............

2. Nominal Flow Parameters-Accelerating Gas

Stream..................

3. Comparison of the Nominal Flow Parameters

for the Primary References to Those for

the Present Investigation--Constant

Velority Gas Stream ..........

4. Comparison of the Nominal Flow Parameters for the Primary Reference (24) to Those

for the Present Investigation--

Accelerating Gas Stream . . . . . . . 154

5. Estimation of the Surface Tension for the

Analysis of the Data Due to Emmons....

Appendix

Table

B.1. Components of the Gas Generator . . . . . 250

B.2. Output Characteristics of the Gas Generator 251

6.1. Average Values of the Pertinent Flow

Parameters During the Experimental Tests

With a Constant Velocity Gas Stream...

6.2. Average Values of the Pertinent Flow

Parameters During the Experimental Tests

With all ficelerating Gas Streali... 
1. Illustration of the Liquid-Film Conling Process . . . . . . . . . . . . 3

2. Illustration of Gas-Vapor Cooling . . . . . 7

3. Comparison of Liquid-Film Cooling and SimpleMass-Transfer Cooling .......... 12

4. Schematic Diagram of Experimental Apparatus . 19

5. Schematic Diagram of Exnerimental Test Section . . . . . . . . . . . . . 21

6. The Experimental Model . . . . . . . . . 26

7. Influence of $m_{1}$ on Film Surface Characteristics--Low Gas Stream Velocity. . 38

8. Influence of $m_{1}$ on Film Surface Characteristics--High Gas Strean Velocity . 39

9. Influence of Surfaca Tension on Film Surface Characteristics .............

10. Influence of $G, u_{g}$, and $M_{0}$ on Film Surface Characteristics--G Constant . . . . . . . 44

11. Influence of $G, u_{g}$, and $M_{0}$ in Film Surface Characteristics--ug Constant.. . . . . . 46

12. Influence of $G, u_{g}$, and $M_{0}$ on Film Surface Characteristics--Mo Constant . . . . . .

13. Rate of Liquid Withdrawal as a Function of Vent Flow Rate for Separator........ 
Figure

Page

14. Example of Experimental Data for Mass

Transfer. Constant $u_{q}$. Hethanol Film

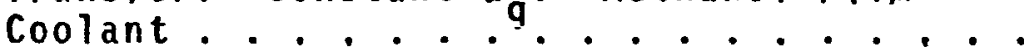

15. Influence of Surface Terision and Liquid Viscosity on Experimental Data for

Mass Transfer

16. Influence of Gas Stream Parameters on Experimental Data for Mass Transfer....

17. Experimental Data for Mass Transfer.

Accelerating Gas Stream. Metháncl and

Hater Film Coolant ...........

18. Comparison of Muximum Liquid Temperature and Boiling Temperatire . . . . . . . .

19. Schematic Illustration of Separate Contributions to Net Rate of Mass Transfer

20. Control Volume for Interfacial Energy Balance................

21. Entrainment Parameter $e_{0}$ as a Function of the Entrainment Group $x_{e}$. . . . . . .

22. Roughness iarameter $r$ as a Function of the Roughness Group $X_{r}$.............

23. Comparison of Simple Theory and Present Theory With Data for Mass Transfer. Constant Velocity Hot Gas Stream. Methanol

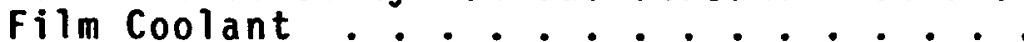

24. Comparison of Simple Theory and Present Theory With Data for Mass Transfer. Constant Velocity Hot Gas Stream. Butanol Film Coolant ..............

25. Comparison of Simple Theory and Present Theory With Data for Mass Transfer. Constant yelucity liut Gas Stream. Hater Film Coolant.............

26. Comparison of Simple Theory and Present Theory With Data for Mass Transfer. Constant Velocity Hot Gas Stream. RP-1 Film Coolani 
21. Comparison of Present Theory With Data fur Mass Transfer. Constant Velocity Cold Gas Stream.............

28. Model for Analysis of Arbitrary FilmCooled Length . . . . . . . . . . . .

29. Matching of Empirical Equation to Analytical Result. $m_{s}^{\prime \prime}=c x^{-1 / 5}$

30. Matching of Empirical Equation to Analyticai Result. $m_{s}^{\prime \prime}=c x . . . . . . . . . .$.

31. Pertinelit Gas Stream Parameters as a Function of the Disiance From the Point of Liquid Injection. Accelerating Gas Stream. Accelerating Gas

32. Model for Analysis of Experimental Data for Mass Transfer. Accelerating Gas Stream.

33. Comparisun of Simple Theory and Present Theory With Data for Mass Transfer. Accelerating Gas Stream................

34. Computed Results for Maximum Liquid

Temperature as a Function of $\mathrm{T}_{g}$ and $p$ for an Air-Water System ...........

35. Comparison of Present Theory With Data for Maximum Liquid Temperature.... . . . . 148

36. Schematic Diagram of Apparatus of Kinney, Abramson, and Sloop ............

37. Comparison of Simple Theory and Present Theory With Data for Mass Transfer Due to. Kinney, Abramson, ard Sloop. 4-inch Diameter Smooth-Surface Tube. Water Film Coolant...............

38. Comparison of Simple Theory and Present Theory $1: 1:$ th Data for Mass Transfe- $n_{1: .:}$ - to Kinney, Abramson, and Sloop. 2-inch Diameter Smooth-Surface Tube. Water Film Coolani 
39. Comiparison of Simple Theory and Preserit

Theory With Data for Mass Transfer Due to

Kinney, Abramson, and Sloop. 4-inch

Diameter Rough-Surface Tube. Water Film

Coolant

40. Nusselt-Reynolds Number Plot of Data Due to Kinney, Abramson, and Sloop. Smooth-

Surface Tubes ...............

41. Nusselt-Reynolds Number Plot of Data Due to

Kinney, Abramson, and Sloop. Rough-

Surface Tube... . . . . . . . . . 173

42. Schematic Diagram of Apparatus of Emmons . . 178

43. Comparison of Simple Theory and Present

Theory With Data for Mass Transfer Due to

Emmons. Various Gas Stream Reynolds

Numbers . . . . . . . . . . . . . .

44. Comparison of Simple Theory and Present

Theory With Data for Mass Transfer Due to

Emmons. Various Film Coolants......

45. Schematic Diagram of Apparatus of Hermann, Leitinger, and Melnik.........

46. Coolant Flow Rate as a Function of the FilmCooled Length and the Surface Tension . .

47. Local Mass Flow Rate as a Function of the

Axial Position in the Nozzle and the Coolant Flow Rate

Appendix

Figure

A.1. Flow Model for Analys is of Gas-Vapor Cooling. Adiabatic Wall............ . . 221

A.2. Comparison of the Present Theory With the Data for Acilabatic-Wall Temperature Due to Kinney, Abramson, and Sloop ......

B.1. Photograph of Experimental Apparatus. Configuration for Tests with Constant Velocity Gas Stream ........... 
Appendix

Figure

B.2. Photograph of Experimental Apparatus.

Configuration for Tests With

Accelerating Gas Stream......... 243

B.3. Photograph of Control Room........ 24?

B.4. Schematic Diagram of Gas Generator..... 249

B.5. Schematic Flow Diagram.......... 256

B.6. Sectional Drawing of Test Section ..... 261

B.7. Cross Sectional Drawing of Test Section . . 262

B.8. Photographs of Test Section ........ . 265

B.9. Sectional Drawing of Separator . . . . . . 267

B.10. Photograph of Separator .......... 268

D.1. Surface Tension as a Furiction of Liquid

Temperature for Liquids of Present

Investigation ............. 275

D.2. Liquid Viscosity as a Function of Liquid

Temperature for Liquids of Present

Investigation .... 276

D.3. Heat of Vaporization as a Function of Liquid

Temperature for Liquids of Present

Investigation .............. 277

D.4. Vapor Pressure as a Function of Liquid

Temperature for Liquids of Present

Investigation ..............

D.5. Surface Tension as a Function of Liquid

Temperature foi th: Liquids Employed by

Emmons.

D.6. Heat of Vaporization as a Function of Liquid

Temperature for the Liquids Employed by

Emmons.................

D.7. Vapor Pressure as a function of Liquid

Temperature for the Liquids Employed by

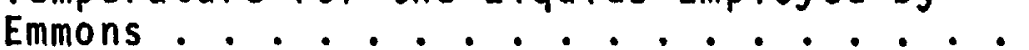


Appendix

Figure

G.1. Data for Mass Transfer. $T_{g}=600 \mathrm{~F}, p=75$ psia. Water Film Coolant . . . . . . 298

G.2. Data for Mass Transfer. $T_{g}=600 \mathrm{~F}, p=150$ psia. Water Film Coolant . . . . . . 299

G.3. Data for Mass Transfer. $T_{g}=400 F, p=75$ psia. Methanol Film Coolant...... 300

G.4. Data for Mass Transfer. $T_{g}=600 \mathrm{~F}, p=75$ psia. Methanol Film Coolant....... 301

G.5. Data for Mass Transfer. $T_{g}=400 \mathrm{~F}, p=150$ psia. Methanol Film Coolant. . . . . 302

G.6. Data for Mass Transfer. $T_{g}=400 F, p=75$ psia. Butanol Film Coolant . . . . . 303

G.7. Data for Mass Transfer. $T_{g}=400 F, p=150$ psia. Butanol Film Coolant ....... 304

G.8. Data for Mass Transfer. $T_{g}=600 \mathrm{~F}, p=75$ psia. Butanol Film Coolant . . . . . 305

G.9. Data for Mass Transfer. $T_{g}=400 \mathrm{~F}, p=75$ psia. RP-1 Film Coolant... . . . . 306

G.10. Data for Mass Transfer, $T_{g}=400 \mathrm{~F}, p=150$ psia. RP-l Film Coolant . . . . . . . 307

G.11. Data for Mass Transfer. $T_{g}=600 \mathrm{~F}, p=75$ psia. RP-1 Film Coolant........ . 308

G.12. Data for Mass Transfer. Cold Flow, $p=75$ psia. Methanol Film Coolant.......... 309

G.13. Data for Mass Transfer. Cold Flow, $p=75$ psia. Water and Butanol Film Coolant...... 310

G.14. Data for Mass Transfer. Accelerating Gas Stream. Water and Methanol Film Coolant. 
Gater, Roger Allen. Ph.D., Purdue University, January 1969. A Fundamental Investigation of the Phenomena That Characterize Liquid-Film Cooling. Major Professor:

Dr. Mel R. L'Ecuyer.

An experimental investigation was conducted to determine the net rate of mass transfer (evaporation plus entrainment) from a thin liquid film to a proximate gas stream. Three different flow situations were investigated: (a) constant velocity, cold gas flow; (b) constant velocity, heated gas flow; and (c) strongly accelerated, heated gas flow. The experiments were conducted in a horizontal test section by passing an air stream over a liquid film formed on the lower surface. The liquid film was $2-i n$. wide and was terminated at a length of $10 \mathrm{in.}$ by a knife-edge capture slot. The flow parameters for the experiments with a constant velocity gas stream were: gas stream temperatures of $40 \mathrm{~F}$, $400 \mathrm{~F}$, and 600F; gas stream static pressures of 75 and 150 psia; gas stream velocities of 40 to $400 \mathrm{fps}$; ard liquid coolants of methanol, butanol, water and RP-I (a hydrocarion fuel). The flow parameters for the experiments with an accelerating gas stream were: stagnation temperature of $400 \mathrm{~F}$; stagnation pressures of $50,75,100,125$ and 150 psia; 
$x v i i$

variation in gas stream velocity of 200 to $660 \mathrm{fps}$ over the liquid film; and liquid coolants of methanol and water.

The measured rate of mass transfer with a heated gas stream was found to be as much as 10 times greater than the rate of mass transfer predicted from the simple (or classical) theory for mass transfer. In most cases, the mass transfer due to the entrainment of buik liquid into the gas stream was the most significant contribution to the overall mass transfer. In those cases where evaporative mass transfer was important, the "effective" roughness of the surface of the liquid film was found to increase the rate of evaporation by as much as 80 percent over that predicted by the simple theory.

All of the data for mass transfer (approximately 600 datum points) was correlated in terms of relatively simple dimensional parameters, with one parameter characterizing the phenomenon of entrainment and a second parameter characterizing the phenomenon of film surface roughness. Of particular significance is the fact that a fifteenfold variation in the liquid viscosity was not found to have any noticeable effect on the iate of entrainment.

An analysis was made to extend the results of the present investigation to the more general case of an arbitrary film coolea length. The utility of the analysis was investigated by analyzing the data reported in three primary references. The agreement between the reported data and the 
xviji

results predicted from the present theory was good, with the agreement being to within the accuracy of the experiniental data in most cases.

Photographs were taken of the surface of the liquid film so that the gross characteristics of the film surface could be studied as a function of the pertinent flow variables for the liquid and gas phases.

Measurements were made of the maximum temperature that a liquid filn obtains for the subject flow situation. A theory was developed for the prediction of the maximum liquid temperature that was in good agreement with the experimental data.

An analysis was made of the heat transfer characteristics for the gas-vapor cooled region immediately downstream of the liquid film. The analysis allowed for compressible gas flow in the presence of a favorable pressure gradient. Consideration of the limited experimental data that are reported in the literature for the subject problem indicated that the analysis was promising. 
1. INTRODUCTION

\subsection{General Discussion}

Liquid-film cooling refers to the introduction of a thin continuous liquid film onto a given surface for the purpose of protecting that wetted surface from thermal damage by a proximate hot gas strean. This technique provides a means of protecting, for example, the internal surfaces of a rocket motor from the injurious effects of the hot propellant gases. The motivation for developing liquid-film cooling technology for rocket motors is due to the use of higher energy chemical propellants, the trend toward higher combustion chamber pressures, and the development of nuclear rocket engines. These newer developments are characterized by a large increase in the heat flux from the working fluid to the walls of the combustion chamber and exhaust nozzle, such that the regenerative cooling of those walls becomes marginal $(1,2,3)$.

In the design of a liquid-film cooled system for a rocket motor, the two principal problems to be considered are (a) that of determining the rate of coolani consumption that will be required to wet, and thus thermally protect, a

* Numbers in parentheses refer to REFERENCES. 
specified internal surface area, and (b) that of determining the effect that the cooling process has on the performance of the system. In the subject investigation, atieniion was concentrated on the first problem only; specifically, with the investigation of the phenomena that characterize the convective exchange of heat and mass between a hot gas stream and a thin liquid film. Although the principal motivation for this research was the interest in liquid-film cooling, the investigation is of a much broader interest because it relates to the basic problem of the interaction of a high velocity gas stream and a thin 1 iquid film, a situation that is frequently encountered in engineering.

\subsection{A Statement of the Problem}

Figure 1 illustrates the process termed liquid-iilm cooling. Presented in the figure is a hot gas stream flowing along a surface $A-B$. A liquid coolant is introduced at station $x=x_{1}$ and is swept downstream over the surface by the gas, thereby forming a continuous protective liquid film. As a result of the evaporation of liquid in the film, together with the possible entrainment of liquid droplets into the gas stream, the liquid film will terminate a some point, say, $x=x_{2}$. The wall region wetted by the liquid will here$\therefore$ be termed the liquid-film cooled region.

Since the gas and vapor mixture which is convected downstream is relatively cool, the wall region imniediately downstream of the liquid film will be insulated to some degree 


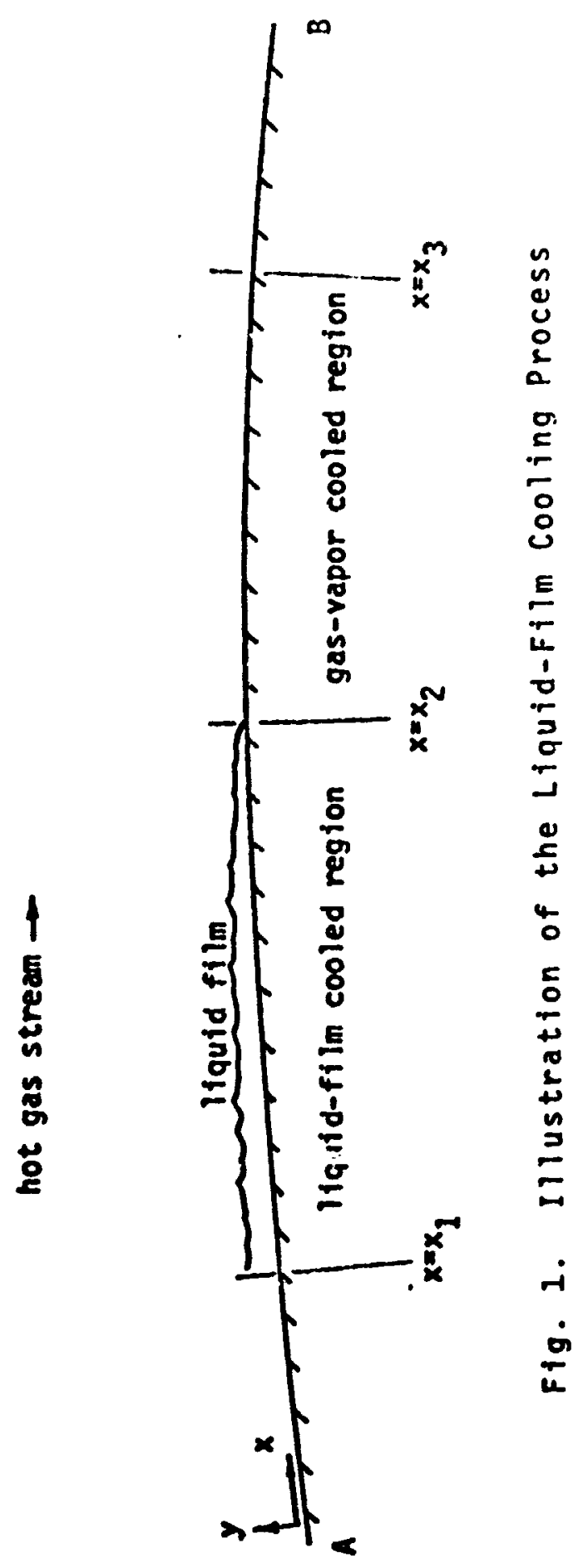

i

I 
from the hot gas stream. It is convenient to define the gas-vapor cooled reaion as that wall region over which the temperature of an adiabatic surface would increase from that for the liquid film at $x=x_{2}$ to the recovery temperature for the mainstream at, say, $x=x_{3}$. The exterit of the gasvapor cooled region so defined is thus a measure of the effectiveness of the gas-vapor cooling process.

The basic analytical and design problem can be stated as that of determining, for given liquid and gas flow parameters, (1) the rate of coolant injection required to estab. lish a desired wetted area, and (2) the degree of the in. sulating effect of the gas-vapor layer downstream of the liquid film. The remaining portion of this discussion is directed toward a riore detailed consideration of each of these two problems.

\subsubsection{The Liquid-Film Cooled Region}

The basic protlem for the liquid-film cooled region is that of being able to predict the wetted surface area as a function of the rate of liquid injection and as a finction of the pertinent flow parameters for both the liquid and gas phases. The solution to this problem is difficult to obtain because of the complex phenomena that characterize the flow or a high velocity gas over a liquid film.

The subject problem is outlined more specifically as follows: For the case where a high velocity, hot gas stream is flowing over a thin (of the order of a few thousandths of 
an inch thick) liquid film, the mass transfer from that film is made up of two fundanentai contributions: (a) the mass transfer that results from the trarsfer of energy to the liquid film and the resultant evaporation of the liquid; and (b) the mass transfer that results from a physical breakdown of the interfacial structure such that iqquid droplets are entrained into the gas stream.

The evaporative contribution to the total mass transfer rate (item (a) above) can be divided into yet two more distinct contributions: (a-1) that contribution which would result if the liquid film surface presented a hydrodynamically smooth, zero velocity boundary to the gas stream; and (a-2) the remaining portion of the evaporative mass transfer which results because the surface of the liquid film is moving at a finite velocity and does rot, in general, present a "smooth" surface to the gas stream. (The surface of the liquid film generally exhibits a wavy or pebbled appearance which should serve to intensify the turbulent transfer of heat and mass near the gas-liquid interface.)

Contribution $(a-I)$ is the same as that prevailing in the case of simple mass transfer*. That part of the total evaporative contribution is relatively amenable to analysis. Reference (4), for example, presents a recent review of the extensive analytical and experimental work performed on that problem of simple mass transfer. Although there still

\footnotetext{
*The process termed herein "simple-mass-transfer cooling" is equivalent to the process that is frequently termed "transpiration cooling" in the published literature.
} 
remain some problem areas in the thorough treatment of simplemass-transfer cooling, the effect of mass addition to a boundary layer upon the rate of heat transfer to the surface has been adequateiy described for the purposes of the present investigation.

No systematic attempt has previously been made to experimentally determine the individual contribution of each of the three aforementioned mass transfer mechanisms (a-I), $(a-2)$, and (b) to the overall mass transfer rate. Although the fundamental problem is that of being able to predict the total mass transfer rate for a particular flow situation, it is evident that in order to develop a satisfactory correlation for that total mass transfer rate, a basic understanding has to be developed of the nature of each of the three contributions discussed above.

\subsubsection{The Gas-Vapor Cooled Region}

Figure 2 serves to introduce the analytical problem for the gas-vapor cooled region. Illustrated in Fig. 2 are the pertinent wall temperature profiles immediately downstream of the terminus of the liquid film. The upper.solid curve presented represents the wall temperature profile for the case where the wall is adiabatic*. The lower solid curve represcints a characteristic Miviiie for the case where the wall is cooled externally. As indicated in the figure, it is convenient to base the heat transfer coefficient for gas-vapor 
not gas stream
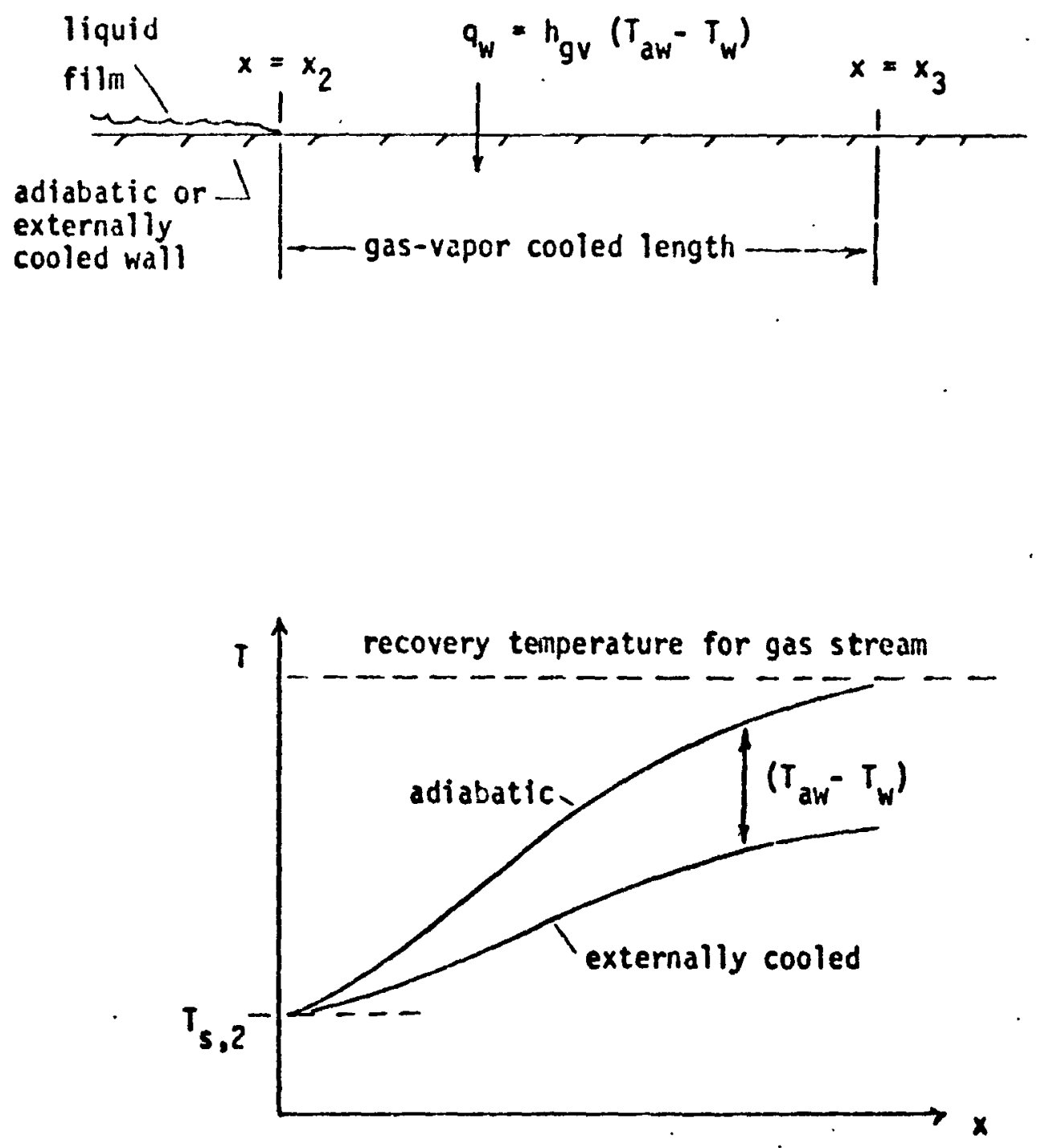

Fig. 2 diustration of Gas-Vapor cuving 
cooling, denoted by $h_{g v}$, on the temperature difference $\left(T_{a W^{-}} T_{W}\right)$. When that is done, the rate of heat transfer to an externally cooled wall is given by

$$
q_{w}=h_{g v}\left(T_{a w}-T_{w}\right)
$$

It follows, therefore, that the basic problem is to determine, for given flow parameters, (1) the adiabatic wall temperature profile, and (2) the local heat transfer coefficient $h_{g v}$.

\subsection{A General Review of the Literature}

Research that has been performed that is pertinent to the subject problem can be conveniently grouped into two principal areas:

(a) research concerned primarily with the investigation of the detajled nature of the flow of a gas over a thin liquid film; and

(b) research concerned with an actual application of liquidfilm cooling.

The first area of research includes the investigation of such fundamental phenomena as interfacial structure and film instability* $(5,6,7,8,9)$, entrainment $(10,11,12)$, welting characteristics $(13,14,15)$, and pressure crop in 
annular, two-phase flow $(16,17)$. In general, those investigations were characterized by ambient pressure and temperature, essentially zero heat transfer rates, and relatively low interfacial shear forces. Such conditions are significantly difierent from those that characterize the typical application of liquid-film cooling. Furthermore, few data exist for the case where the liquid phase was not water. Although the experimental advantages of using water as the liquid phase are obvious, the fact remains that few other liquids have physical properties (particularly surface tension) that are similar to those of water. Considerable doubt thus exists regarding the application of results that were obtained with water as the liquid phase to other liquids. A detailed review of the literature in this first area of research is not presented here because of the question as to the relevance of the results--particularly in a quantitative sense--to the present investigation. The interested reader is referred to the excellent reviews presented in references $(5,10,18)$. However, several specific references are made to those experimental results throughout this report when that reference gives some insight into the interpretation of the experimental results of the present investigation.

The research of the second area (namely, applications of liquid-film cooling) is characterized by relatively high rates of heat and mass transfer between the gas stream and 
the liquid film $(19,20,21,22,23,24)$. The objectives of those research prograns were basically (a) to establish the feasibility of liquid-film cooling the thrust chanber and/or nozzle of a rocket motor; and (b) to determine the relationship between the rate of coolant consumption and the resultant wetted surface area as a function of the gas stream Reynolds number, the gas stream pressure arid temperature, the properties of the liquid coolant, etc. Attempts to develop empirical correlations for the experimental data have met with only limited success. The apparent reason for the lack of success has been the failure to incorporate into those correlations the influence of the interfacial phenomena* on the rate of mass transfer from the liquid film to the gas strean $(25,26)$. Moreover, theoretical analyses developed for liquid-film cooling (e.g., 20, 21) have either lacked agreement with the experiniental data or have resulted in correlations that were not justified physically; the previous analyses for liquid-film cooling have been critically reviewed in Ref. (26). The failure of the theoretical analyses is apparently due to the fact that they have assumed a direct correspondence between the problem of liquidfilm cooling and that of simple-mass-transfer cooling. Analytical models which had resulted in satis-actor: analyses for the case of simple-mass transfer were also assumed to describe the heat and mass transfer characteristics for the 
case of liquid-film cooling. Figure 3 aids in an explanation of the motivation for such an approacin. Illustrated in the figure is a control surface "s" placed at the surface of an evaporating liquid film and at the surface of a smooth porous wall through which mass is being transferred into the gas stream. Assume that for each case the flow! parameters, the boundary conditions, and the rate of mass transfer across the control surface into the gas stream are the same. It is then reasonable to argue that the following procedure can be employed to analyze the problem of liquid-film cooling. Thus

(a) The simple theory for mass transfer is employed to calculate the rate at which energy is transferred across the control surface "S" to the liquid film. The simple theory suggests that the rate of heat transfer to the liquid film decreases as the rate of mass transfer from the liquid film into the gas stream increases. That phenomenon is sometimes referred to as thermal "blockage".

(b) The rate of mass transfer from the liquid film into the gas stream is determined by writing an appropriate energy balance across the gas-liquid interface*. This step is coupled to step (a) through the "blockage" phenomenon so that there is only one value for both the rate of heat transfer to the iiquid film and the rate of mass transfer into the gas phase that is predicted for any particular

* See Subsection 3.1 .2 for a discussion on the interfacial energy balance. 


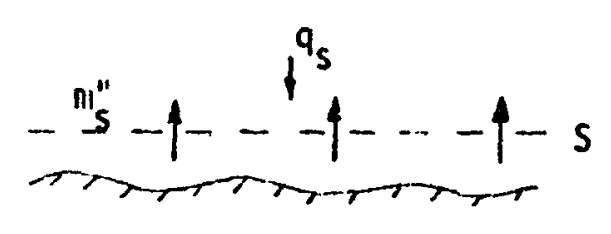

liquid film

liquid-film cooling

$$
\begin{gathered}
\text { simple-mass-transfer } \\
\text { cooling }
\end{gathered}
$$

Fig. 3 Comparison of liquid-Film Cooling and Simple-Mass-Transfer Cooling 
situation. The following paragraph discusses how the experimental data of the primary references agree with the foregoing argument.

Emmons (20) concluded from his experimental investigation that the analogy between simple-mass-transfer cooling and liquid-film cooling was valid. Emmons suggested that the rate of heat transfer to an evaporating liquid film was always less than the rate of heat transfer to a dry wall, all other conditions being the same. He attributed this, of course, to thermal blockage. However, that conciusion drawn by Emmons is contradicted by the experimental data obtained by other investigators*. Kinney, Abramson, and Sloop (19) have reported experimental data showing that the rate of heat transfer to a liquid film can be substantially greater (by a factor of 3 or more) than the rate of heat transfer to a dry wall**. Luikov (27) obtained similar results from well controlled experiments in which extreme care was taken to ensure identical gas flow conditions and surface temperatures for (a) dry surface and (b) liquid surface test sections. The coefficient of heat transfer to the liquid film was consistently 20 to 60 percent greater than the coefficient of heat transfer to the dry wall. Hermann, Leitinger and Melnik (24) considered the problem of film-

* Evidence is presented in Section 4.4 of this report that the data of Emmons do not, in fact, support the analogy between simple-mass-transfer and liquid-film cooling. **The data of Ref. (19) are analyzed in Section 4.3. 
cooling a convergent-divergent nozzle with water*. They found that the amount of coolant required to completely wet the convergent portion of the nozzle was roughiy 2 times that predicted by the theory for simple mass transfer. They also estimated that the amount of coolant required for the divergent portion of the noczle was 3.3 times that predicted by the simple theory**.

The foregoing discussion raises a serious question regarding the adequacy of a simple-mass-transfer model for the analysis of liquid-film cooling. The difficulty of obtaining an adequate empirical correlation or developing a satisfactory theoretical analysis for liquid-film cooling on the basis of the previously reported data for the rate of mass transfer from a thin liquid film to a high velocity gas stream is due primarily to:

(a) the limited amount of data that has been obtained for conditions similar to those encountered in an application of liquid-film cooling;

(b) the contradictory trends exhibited by the data of the primary references;

(c) the possibility of appreciable experimental error due to the severity of the fiow parameters investigated in many cases; and

* The data of Ref. (24) are analyzed in Section 4.5. **The discussion of section 4.5 (in particular, that of Subsection 4.5.5) suggests that the estimates by Hermann, et a 1. (24) of the mass transfer rates for both the subsonic and supersonic portion of a nozzle cannot be generally applied. 
(d) the scarcity of data for film coolants other than water.

The present research program vas initiated because of the incomplete understanding of the phenomena that characterize liquid-film cooling, and because of the limited information that could be obtained from the existing literature.

\subsection{The Scone of the Investigation}

The subject investigation had the primary objective of experimentally determining the net rate of mass transfer from a thin liquid film to a high velocity gas stream under conditions of relatively high pressure, temperature, and interfacial shear forces with significant variations in the physical properties of the liquid phase. Two cases were investigated: (a) the case where the velocity of the gas stream was essentially constant over the length of the film-cooled region, and (b) the case where the gas stream was strongly accelerated over the wettcd surface. The latter case was investigated because of the interest in the application of liquid-film cooling to cool the exhaust nozzles of high-energy propulsion devices.

A limited number of experiments were conducted for Case (a) in which the gas stream was not heated. These experiments served to (I) isolate the mass transfer that was due to entraic:.:::: : From the mass transfer that was due to the evaporation of the liquid in the film, and to (2) significantly extend the range of values for the pertinent flow parameters that was investigated in the present 
investigation.

A correlation was developed for the mass transfer data*, isolating the contributions to the total mass transfer rate that were due to both (a) the simple mass transfer, and (b) the interfacial phenomena. The data correlation gives an explicit expression for the net rate of mass transfer from a thin liquid film to a high velocity gas stream in terms of the pertinent variables for both the gas and the liquid phases.

The maximum temperature that the liquid film obtains when $i t$ is introduced onto the surface of an adiabatic wall was measured for each experimental test that was conducted. A simple theory was developed for the prediction of the maximum film temperature that was in good agrecment with the experimental data. The theory can be employed to estimate the maximum liquid-film temperature for the general case where the film temperature is not a known parameter.

A number of still photographs were obtained in the investigation for the purpose of making some qualitative observations about how the pertinent liquid and gas stream parameters infiuence the character of the interfacial structure. Those observations were utilized in the development of the aforementioned correlation for the experimental mass transfer data.

\footnotetext{
* Approximately 600 datum points are reported herein for the rate of mass transfer irom a thin liquid film to a high velocity gas stream.
} 
The utility of the design formula (i.e., thi correiation for mass transferl that was developed for liquid-film cooling was investigated by attempting to correlate the published data of the primary references $(19,20,24)$. In many cases, the values of the pertinert flow parameters le.g., gas stream temperature, pressure, velocity and filmcooled length) investigated by those refarences differed substantially from those of the subject investigation. Analysis of those data, therefore, gave considerable insight into the validity of the design formula proposed herein for liquid-film cooling.

A subordinate objective of this investigation was to present an analysis for the gas-vapor cooled region immediately downstream of the terminus of the liquid film. The analysis presented is essentially the same as that previously reported by Gater, L'Ecuyer, and Warner (26). Since that origina! presentation, however, the andysis has teen further evaluated and additional comments regarding its usefuiness can be made. 


\subsection{The Basic Experimental Apparatus*}

Figure 4 illustrates schematically the experimental apparatus that was employed in the investigation. The basic components of the apparatus were a hot gas generator and a two-dimensional mixing section, ar adiabatic approach section, a film-cooled test section, and a variable area exhaust nozzle.

The hot gas generator employed methyl alcohol and air as the reactants. Because so much diluent air was added to the combustion gases before they entered the experimental tunnel, the properties of the gas stream were assumed to be the same as those for dry air.

Prior to entering the test section, the gas stream passed through an assortment of fine mesh screens in the mixing section, thereby appreciably reducing the turbulence level and the non-homogenities of the flow: The gas then passed through a 36-inch long adiabatic approach section so that the remaining irregularities in the gas stream had a chance tu dissipate and the velocity boundary layer had an 


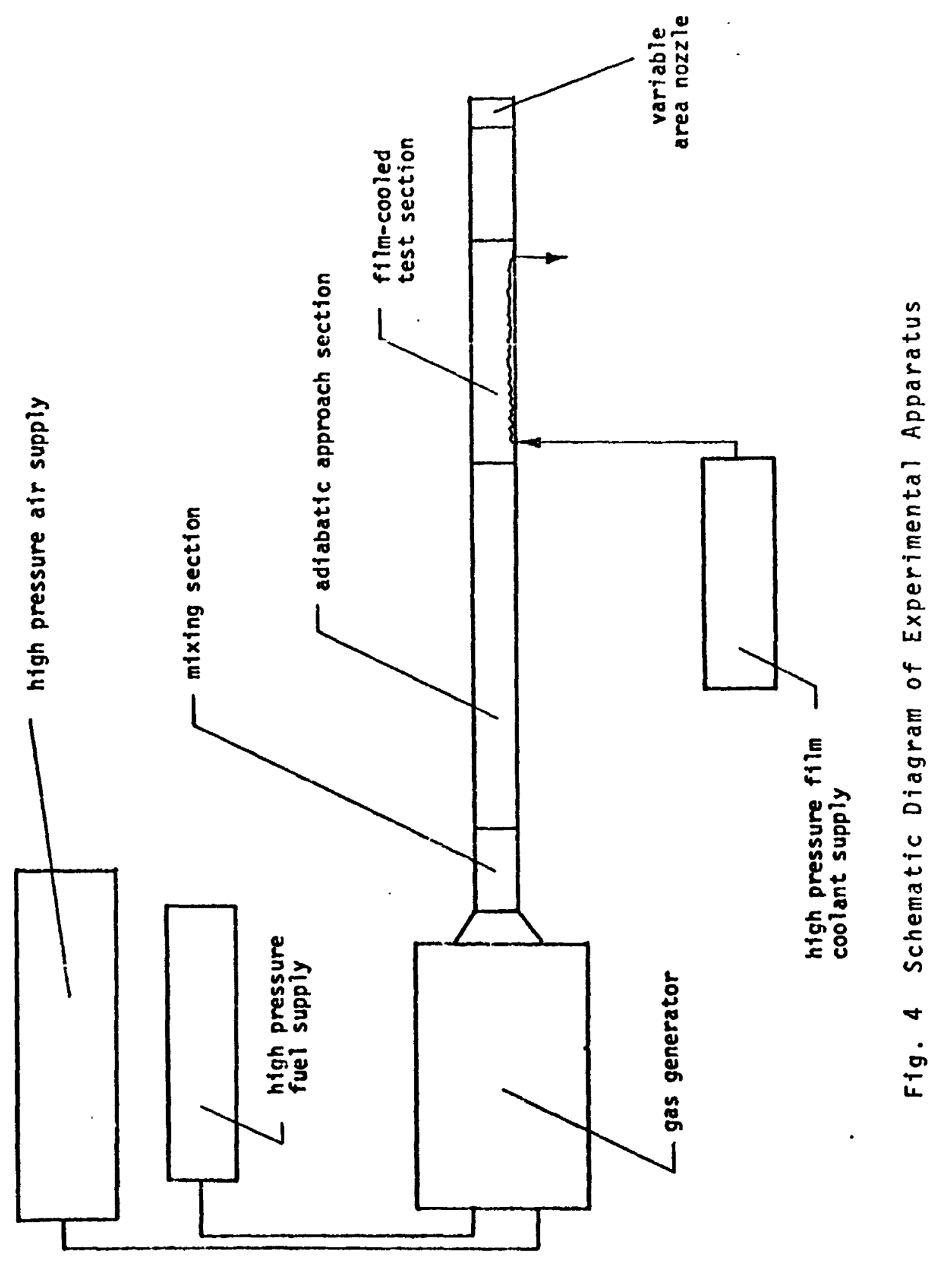


opportunity to develop before the liquid film was introduced. (The thickness of the velocit, boundary layer at the point of liquid injection was on the order of 0.2 to 0.4 inches.) The inside surface of the bottom wall of the approach section was surface ground so that a hydrodynamically smootr. boundary was presented to the developing boundary layer.

Figure 5 shows schematically the test section employed in the investigation. The nominal internal dimensions of the test section are 2 in. by 5.5 . in. cross section by 18 in. long. Mounted in each side wall of the test section were 6 circular Pyrex windows of 1 in. 0.D. A single oblong Pyrex window was mounted in the top wall of the test section. These windows permitted visual and photographic observation of the liquid film. The photographic technique that was employed is illustrated in Fig. 5. The test plate to which the liquid film was confined was recessed into the bottom wall of the test section. The liquid coolant was introduced onto the test plate through an $1 / 8-i n$. thick piece of "regimesh" that was $1 / 2$ in. long and 2 in. wide. This injector design provided a uniform distribution of couiant and also kept the normal component of velocity for the liquid at the injector face to something less than one ft per sec. The liquid film was confined to the central portion of the test plate by means of a trough that was machined into the plate. The trough was $2 \mathrm{in}$. wide and $0.030 \mathrm{in}$. deep, and it extended from the point of liquid injection to a point 


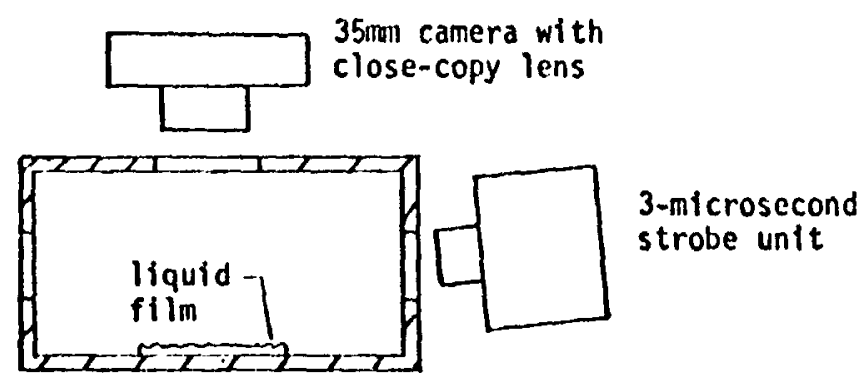

photographic technique

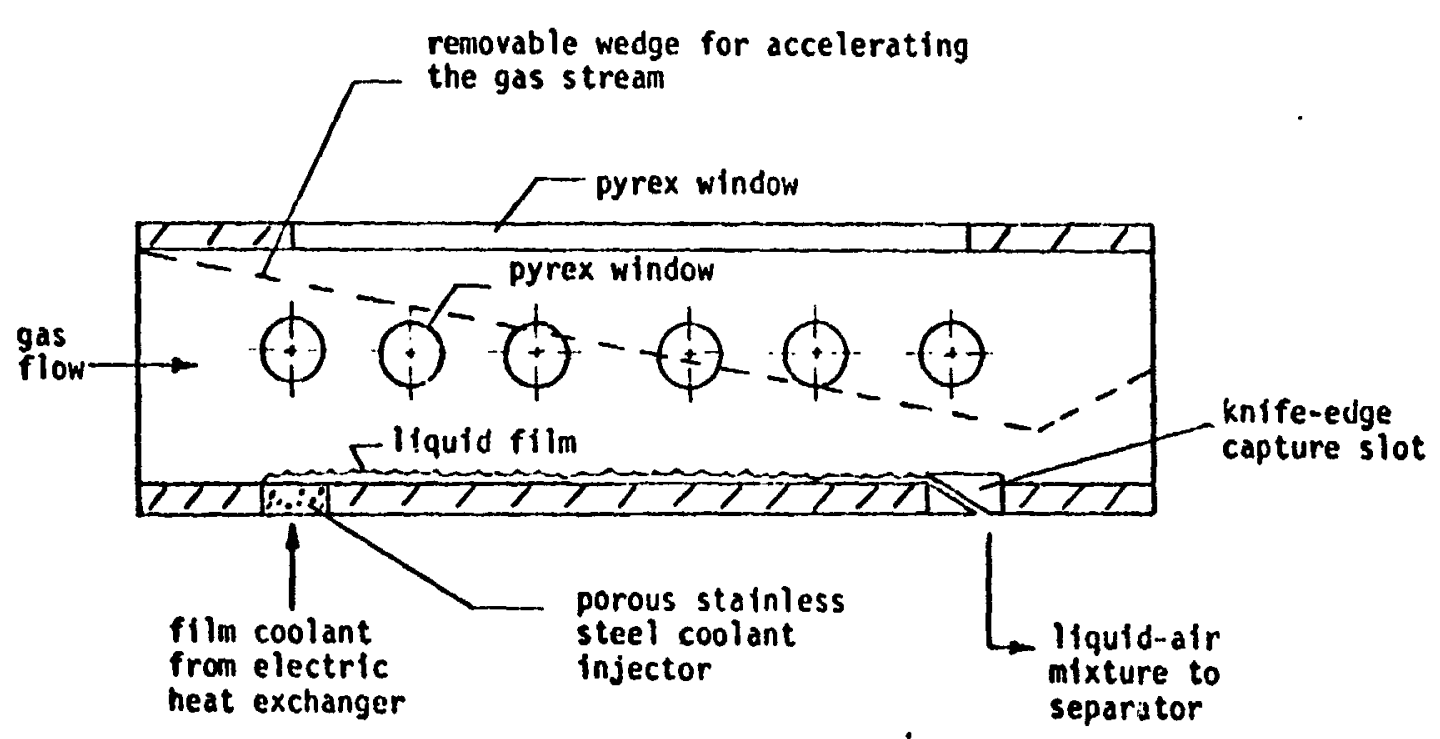

Fig. 5 Schematic Diagram of Experinental Test Section 
10 inches downstream. At that point the liquid film was mechanically terminated by means of a knife-edge capture slot. The projected area for the capture of the liquid film was 2.25 in. wide* by $0.060 \mathrm{ill}$. high**. In preliminary experimentation with the effectiveness of the capture slot technique, it was found that it was necessary to allow some of the gas stream to bleed through the slot with the liquid. Attempts to withdraw only the liquid film resulted in spill. age of the liquid film over the capture slot. The significance of this bleed gas flow is discussed in section 2.5 . In addition, it was visually observed that a small amount of liquid frequently spilled around the outside corners of the capture slot. This was particularly true if the rate of liquid injection was very high. To eliminate that spillage, the streamlines of the gas flow were made to converge slightly in the immediate neighborhood of the capture slot. Then, because the flow behavior of the liquid film was foun. to be strongly affected by the flow pattern of the gas stream, the liquid film was made to converge slightly toward the center of the capture slot thereby preventing spillage of liquid around the corners of the slot. For most of the experimental tests in which the gas stream velocity was essentially constant over the length of the wetted test

* 0.25 inch wider than the liquid film * a approximately an order of magnitucie greater than the maximum thickness that was investigated for the liquid film 
plate, the convergence of the streamlines for the gas flow was accomplished by replacing the last circular Pyrex window in each side wall of the test section with an 1/2-in. thick by 1 -in. O.D. stainless steel plug to which was welded an 1/8-in. thick by $1-i n$. wide by 1-1/2-in. long stainless steel plate. The degree to which these plates deflected ine gas stream was regulated by rotating the plates, thereby permitting the projected area of each of the plates to be varied from $1 / 8$ in. by $1-1 / 2$ in. to 1 in. by $1-1 / 2$ in. These plates were normally adjusted so that the liquid film would converge from a width of $\hat{z} i \cdots s$ at a point approximately $1 / 2$ inch upstream of the leading edge of the capture slot to a width of $1-1 / 2$ inches at the capture slot. The behavior of the liquid film for the remainder of the tests that employed a gas stream of constant velocity and for all of those tests where the gas flow was accelerated over the test plate was controlled by mounting two pieces of $1 / 32-$ in. thick stainless steel sheet directly on the test plate on each side of the capture slot. These sheets were formed so that they protruded $1 / 4 \mathrm{in}$. into the gas stream and caused the gas flow (and, therefore, the liquid film) to converge toward the center of the capture slot in the manner as previously described. Both of the aforementioned control devices were equally effective in the control of the behavior of the liquid film in the immediate neighborhoud of the capture slot. 
The gas ard liquid mixture that was withdrawn from the test plate was passed through a cyclone separator. The gas phase was vented off and the liquid phase was collected in a beaker. Thus, by measuring the rate of liquid injection and the rate of liquid withdrawal, the net rate of mass transfer from the liquid film to the gas stream was determired. Also determined during the course of each mass transfer measurement were the teinperatures for the liquid at the point of injection and at the point of withdrawal. The liquid temperatures were measured with thermocouples and they were employed to accurately specify the energy balance for the liquid film and to determine the values for the pertinent physical properties for the liquid phase.

For those experiments where the gas stream was accelerated over the length of the wetted test plate, the acceleration of the gas stream was accomplished by inserting an aluminum wedge into the top of the test section (see the dashed line in Fig. 5). Five static pressure taps were installed in the aluminum wedge so that the static pressure (and, therefore, the gas stream Mach number, velocity, and static termperature) could be determined as a function of the coordinate $x$. In those experiments the nozzle was removed from the test section and the flow was choked at the minimum area formed by the wedge.

In the subject investigation, the experimental apparatus was designed so that extraneous heat transfer and fluid 
dynamic effects could be safely neglected, rather than trying to correct for them analytically. The liquid film was injected so as to wet only the center 2 inches of the 5.5-in. wide lower surface of the test section, thereby permitting the influence of the complex flow phenomena for the corners of the rectangular duct on the rate of convective heat transfer to the liquid film to be neglected. In establishing an energy balance for the liquid film, it is difficult to determine the amount of energy transferred through the wetted test plate to the liquid film in a lateral direction. Therefore, in order to substantially reduce the lateral conduction of heat through the test plate to the liquid film, two insulating grooves $(0.060 \mathrm{in}$. wide) were cut into the underside of the test plate. The grooves extended to within $0.035 \mathrm{in}$. Of the surface that was wetted by the hot gas, and were placed just outside the region that was wetted by the liquid film. The insulation grooves, extending from the poilt of liquid injection to the point of liquid withdrawal, increased the resistance of the plate to lateral heat conduction by roughly a factor of 50 .

\subsection{The Experimental Model}

Figure 6 illusirates schematically the experimental model for the subjeit irvestigation. The figure presents a high velocity, hot gas stream flowing over a horizontally mounted flat plate. The film coolant was injected at a 


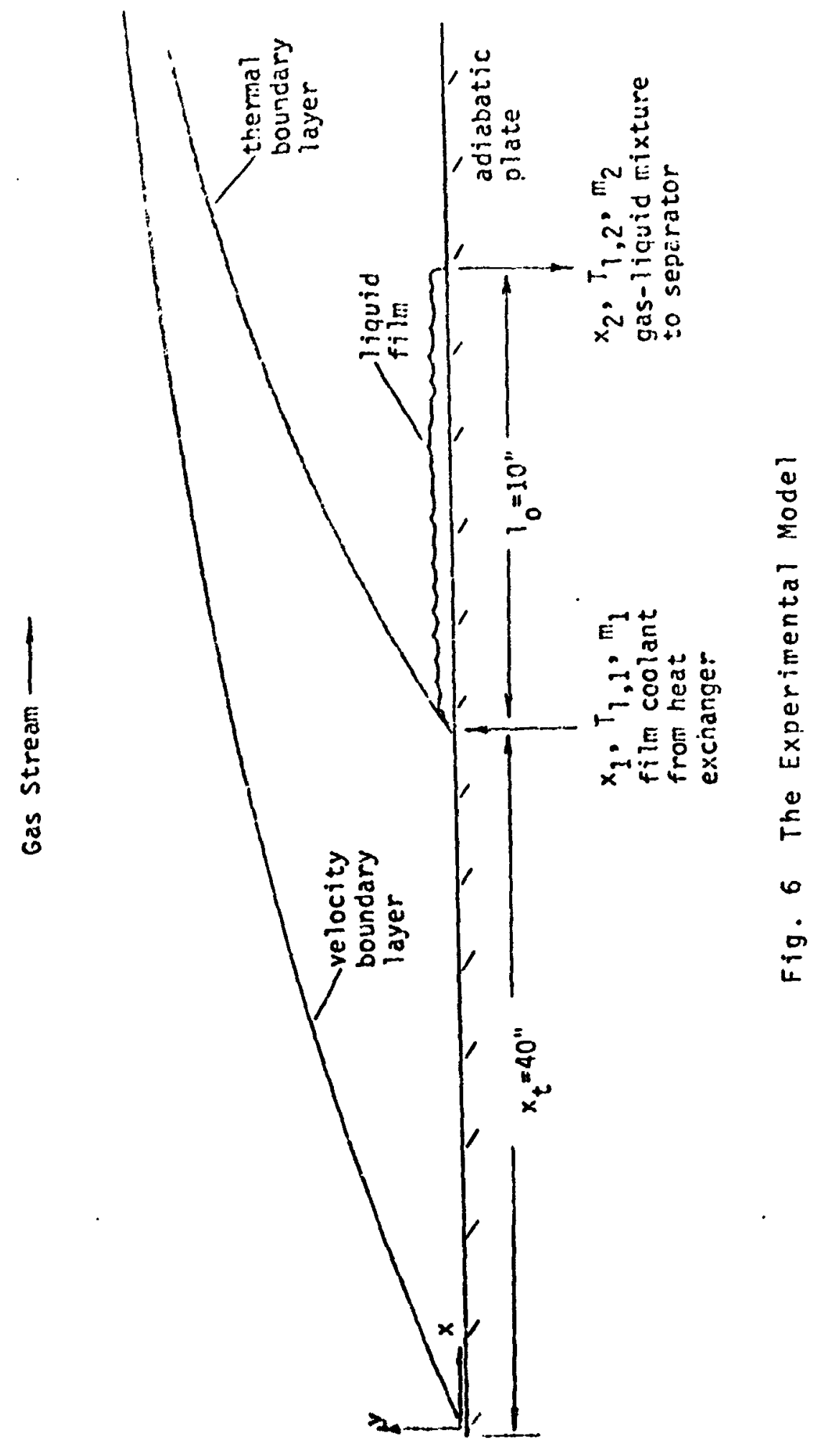

I

1

1

I

1

1

1

1

I

I 
rate $m_{1}(1 \mathrm{~b} / \mathrm{sec}-f t) *$ at $x_{1}=40$ inches and was withdrawn at a rate $m_{2}(1 \mathrm{~b} / \mathrm{sec}-f t)$ at $x_{2}=50$ inches. The film-cooled length, denoted by $1_{0}^{* *}$, was equal to 10 inches throughout the investigation.

The gas stream flow conditions that were investigated were such that the velocity boundary layer developing on the flat plate could be treated as being fully turbulent from the leading edge of the plate. The wall surface upstream of the point of liquid injection was essentially adiabatic so that a thermal entrance lengti $x_{t}=x_{1}=40$ inches was estallished. The thickness of the velocity boundary layer at the poirt of liquid injection was on the order of $0.2 \mathrm{in}$. to $0.4 \mathrm{in}$. Due to the fact that a velocity boundary layer developed on each wall of the experimental tunnel, a correction had to be made in the calculation of the gas stream velocity to account for displacement effects. The calculations showed that the gas stream velocity generally increased by approximately 5 percent in going from $x=\dot{u}$ to $x=x_{t}$. For those investigation. in which the gas stream was accelerated, the acceleration began at a point 6 inches upstream of the point of liquid injection and continued to a

* The units $(1 \mathrm{t} / \mathrm{sec}-\mathrm{ft})$ follow from dividing the rate of liquid injection (Ib/sec) by the width of the injector $(f t)$.

** In general, the film-cooled length will be denoted by

"I". The term 10 refers to the special case of the present investigation where the film-cooled length was fixed at 10 inches. 
point 2 inches divinstream $0 ;$ the end of the film coole: plate.

As indicated in Section 2.1, the liquid film was terminated by means of a knife-edge capture slot. Thus, ur.like previous investigations of liquid-film cooling, the filmcooled length for the present investigation was a fixed parameter. That characteristic of the experimental model allowed the influence of the rate of liquid injection on the cooling process to be investigated independently of the other flow Darameters. Moreover, it enabled the wetted surface area to te accurately determined so that the experimental data could be properly evaluated. In previous experiments (e.g., Refs. 20-24), the wetied surface area was determined by injecting a film of known width and then estimating the average film cooled length by means of therinocouples that were embedded into the test section wall. However, in such experiments it has been found that it is very difficult to obtain a uniform termination of the liquid filn $(19,20,28)$. That is, when the film becomes progressively thinner as a result of the transfer of mass from the film to the gas stream, it reaches a point where surface tension effects and/or flow non-uniformities in the gas stream cause the lirilid filn to braak down locally. It is, therefore, generally very difficult to properly interpret such exte.nal wall temperature measurements.

An experimental technique that was introduced into the subject investigation was that of preheating the liquid 
before injection to the maximu tempercture that the film assures when it is in equilibrium with the gas stream. (When the wetted surface is diabatic, that maximum film temperature is essentially the wet-buib temperature for the liquid.) The advanatages of this experimental technique are essentially twofold: First, because the physical properties for the liquid phase are rather strong functions of the liquid temperature, it is much easier to accurately specify the values for the physical properties of the liquid if the liquid film is isothermal. The second advantage to preheating the liquid before injection is that the energy balance at the gas-liquid interface can then be accurately specified. In previous experimental investigations only an "average" eriergy balance for the entire film could be prescribed because the liquid was incroduced at a relatively low temperature, thereby making it impossible to ascertain what portion of the local heat transfer resulted in an increase in the sensible enthalpy of the liquid and what portion resulted in the evaporation of the liquid. To determine the local rate of heat transfer to the liquid film, however, it is necessary that the local energy balance be prescribed more accurately. The problem of specifying that energy balance is simplified considerably by injecting the liquid at the maximum temperatune it will assume when it is in thermodynamic equilibrium with the hot gas stream.

Due to limitations in the heating capacity of the heat 
exchanger that was empioyed, the liquid could not always be preheated completely to the maximum film temperature. But the liquid was always injected at a temperature substantially greater than the ambient temperature so that the aforementioned advantages to the preheating of the liquid coolant werc largely realized.

\subsection{The Experimental Parameters}

In order to minimize the experimental error and to obtain experimental data for mass transfer of sufficient accuracy to elucidate the phenomena and the parameters of importance, some sacrifice had to be made in the severity of the flow parameters that were investigated, especially the gas stream temperature. A number of datum points exist from previous investigations for the case of relatively high gas stream temperatures (to $4100 R$ ), but the accuracy of those datum points has necessarily suffered because of the measurement problems involved. Moreover, such severe gas stream temperatures place a physical limitation on the number of experimental datum points that can be obtained with a reasonable degree of effort. For those reasons, therefore, since both accuracy and quantity were badly needed for experimental data on liquid-film cooling, the gas strean temperature for the suhject research program was limited to 600F. However, this temperature still resulted in an appreciable rate of evaporation of the liquids investigated because of the relatively high gas stream Reynolds numbers 
that were employed.

As mentioned previously, experiments were conducted for both the case where the gas stream velocity was essentially constant over the length of the film-cooled test section and for the case where the gas stream was accelerated over the length of the wetted test section. Moreover, a limited number of experiments were conducted for the first case for which the gas stream was not heated. Those experimental tests are termed herein the cold-flow experiments.

The 1 iquids employed in the investigation were water, methanol, butanol, and RP-I (a hydrocarbon fuel that is essentially kerosene). These liquids resulted in a rather wide variation in the pertinent physical properties*. For example, the liquid viscosity was varied by roughly a factor of $15: 1$, the surface tension by $3: 1$, and the heat of vaporization by $4: 1$. The significant variation in the physical properties for the liquid phase enabled an accurate determination of the influence that each property exerted on the heat and mass transfer process.

Table 1 summarizes the nominal flow parameters for those experiments in which the velocity for the gas stream was essentially coristant over the length of the wetted test plate. The table also serves to introduce some pertinent

*Appendix D preserts the heat of vaporization, the liquid viscosity, the surface tensicn, and the vapor pressure as a function of the liquid temperature for the liquids that were investigated. 
Table 1

Nominal* Flow Parameters--Constant Velocity Gas Stream

$T_{g}$, Gas Stream Static Temperature

Hot Flow

$400 \mathrm{~F}, 600 \mathrm{~F}$

Cold Flow

$40 \mathrm{~F}$

p, Gas Stream Static Pressure

75 psia, 150 psia

$u_{g}$, Gas Stream Velocity

Hot Flow

$50-400$ fps

Cold Flow

40-200 fps

Re, Gas Stream Reynolds Number

$10^{6}-10^{7}$ per ft

1.0. Film Cooled Length

10 inches

Liquid Film Coolants

Hater

Methanol

Butanol

RP - I

(Hydrocarbon Fuel)

* See Table G.l of Appendix G for a Tabulation of the Specific

Flow Parameters for Eaci Experimental Test With a Constant

Velocity Gas Stream. 
notation that is employed throughout the remainder of this report.

Table 2 summarizes the nominal values of the pertinent flow parameters for the experimental tests in which the gas flow was accelerated over the wetted test plate.

\subsection{Photographic Data for the Film Surface Characteristics}

A number of photographs of the surface characteristics of the liquid film were obtained for a significant range of values of the pertinent iqquid and gas flow parameters. A $35 \mathrm{~mm}$ camera with a close-copy lens, together with a 3microsecond strobe unit for the light source, was employed to photograph the surface of the liquid film. The camera was focused on the surface of the liquid film through the Pyrex window that was mounted in the top wall of the test section (refer to Fig. 5). The strobe unit was focused on the surface of the liquid film through one of the side windows in the test section. All of the photographs presented herein were taken at a point 6 inches downstream of the point of liquid injection.

The surface of a thin liquid film over which flows a high velocity gas stream is generally characterized by a large number of small scale disturbances (or waves) which are essentially three dimensional in chararter These small scale disturbances give the surface of the liquid film a "pebbied" or "cross-hatcrad" appearance. If large scale disturbances (instability waves) develop, they travel over 
Table 2

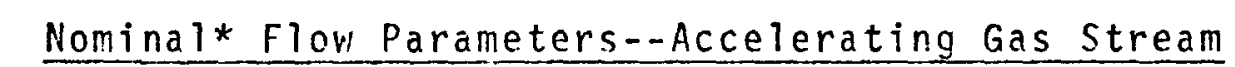

$T_{g}^{0}$, Gas Stream Stagnation Temperature $400 \mathrm{~F}$

$\mathrm{p}^{0}$, Gas Stream Stagnation Pressure $\quad 50,75,100,125$, 150 psia

$u_{g}$, Gas Stream Velocity** 200-660 fps

p, Gas Stream Static Pressure** $50-40$ psia

$75-60$ psia

$100-82$ psia

$125-105$ psia

$150-123$ psia

$M_{a}$, Gas Stream Mach Number** 0.14-0.51

$1_{0}$, Film Cooled Length 10 inches

Liquid Film Coolants Hater

Methanol

* See Table G.2 of Appendix G for a Tabulation of the Specific Flow Parameters for Each Experimental Test With an Accelerating Gas Flow.

**The Numbers Indicate the Variation in the Indicated Parameter Over the Length of the Film Cooled Plate. 
this pebbled substructure at a velocity which is greater than the velocity of the smaller scale disturbances (10). A number of investigaturs (e.g., 19, 29-31) have proposed a criterion for the onset of these large scale disturbance waves on the surface of the liquid film. They have proposed that if the Reynolds number for the liquid film exceeds a certain critical value, then the large scale disturbance waves will develop on the surface of the liquid film. Furthermore, it has been suggested $(e . g ., 9,20,21,22,29,30)$ that appreciable entrainment of liquid from a liquid film into a high velocity gas stream should occur only if the liquid-film Reynolds number exceeds that critical value. First it should be commented that the photographic data of the present investigation did not allow the suggested criterion for film instability to be evaluated. It should also be noted, however, that the data for mass transfer (discussed later in Section 2.5) did not confirm the existence of a critical liquid-film Reynolds number with reference to the phenomenon of entrainment. In fact, it was observed that the liquid viscosity $\mu$, (which enters into the definition of the liquidfilm Reynolds number) had no measurable influence on the raie of mass transfer from a thin liquid film to a high velocity gas stream. Indeed, it is significant that the experimental data for mass transfer could be readily correlated without making any direct reference to the phenomenon of film instability. 
The remainder of this section discusses how the pertinent flow parameters for the liquid and gas phases influence the gross structural characteristics of the surface of the liquid film. A limited number of photographs of the surface of the liquid film are presented to illustrate sevcral important points. It should be nuted, however, that the observations that are made with reference to the influence of the pertinent flow parameters on the film surface characteristics are the result of $\therefore$ analys is of nearly 200 such photographs, and not just the analysis of those photographs that are presented herein. These observations will be pertinent to the correlation that is developed in section 3.1 for the expe imental data on mass transfer.

Subsection 2.4.1, which follows, discusses how the surface rharacteristics of a liquid film are influenced by the parameters for the liquid phase; namely, the rate of liquid injection $m_{\perp}$, the liquid viscosity $\mu_{1}$, and the surface tension 0 . Subsection 2.4 .2 discusses how the surface characteristics are dependent on the gas stream velocity $u_{g}$, the gas stream mass velocity $G$, and the momentum parameter $M_{0}$, where the parameters $G$ and $M_{0}$ are defined by

$$
G=\rho_{g} u_{g}\left(1 b / f t^{2}-s e c\right)
$$


and

$$
M_{0}=\rho_{g} u_{g}^{2}\left(1 b_{f} / f t^{2}\right)
$$

respectiveíy.

\subsubsection{The Influence of the Liquid Parameters} on the Film surface Characteristics

The purpose of the present section is to discuss how the following parameters influence the characteristics of the liquid film surface: (a) the rate of liquid injection $m_{1}$, (b) the liquid surface tension $\sigma$, and (c) the liquid viscosity $\mu_{1}$.

Rate of Liquid Injection $m_{1}$. Figures 7 and 8 present ohotographs of a l-inch-square surface element of the liquid film. The pertinent flow parameters are listed in the figures for each photograph. The photographs shown in Fig. 7 were obtained for a case where the gas stream velocity had ". the relatively low value of $35 \mathrm{ft} / \mathrm{sec}$. Figure 7 (a) illustrates that for the low liquid flow rate of $0.0144 \mathrm{lb} / \mathrm{sec}-\mathrm{ft}$, the film surface is characterized by a uniform distribution of essentially two dimensional waves. Figure 7 (b) demonstrates that as the liquid flow rate is increased to 0.0822 $1 \mathrm{~b} / \mathrm{sec}-\mathrm{ft}$, with the other parameters remainirg constant, the interfacial structure becomes less ordered. However, that particular change in the character of the liquid film surface with increasing rate of liquid injection was observed only 
flow direction -

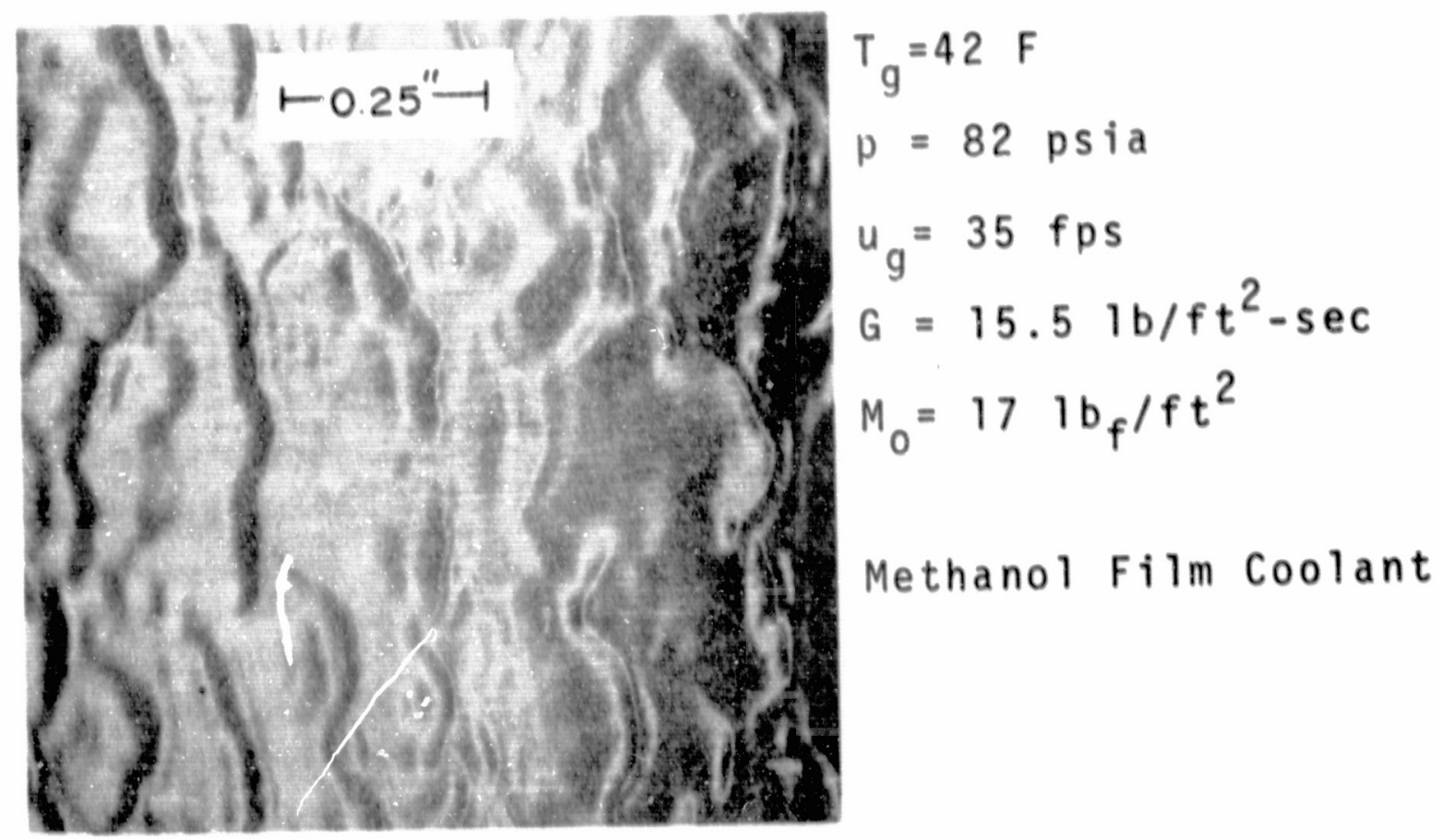

(a) $m_{1}=0.01441 \mathrm{~b} / \mathrm{sec}-\mathrm{ft}$

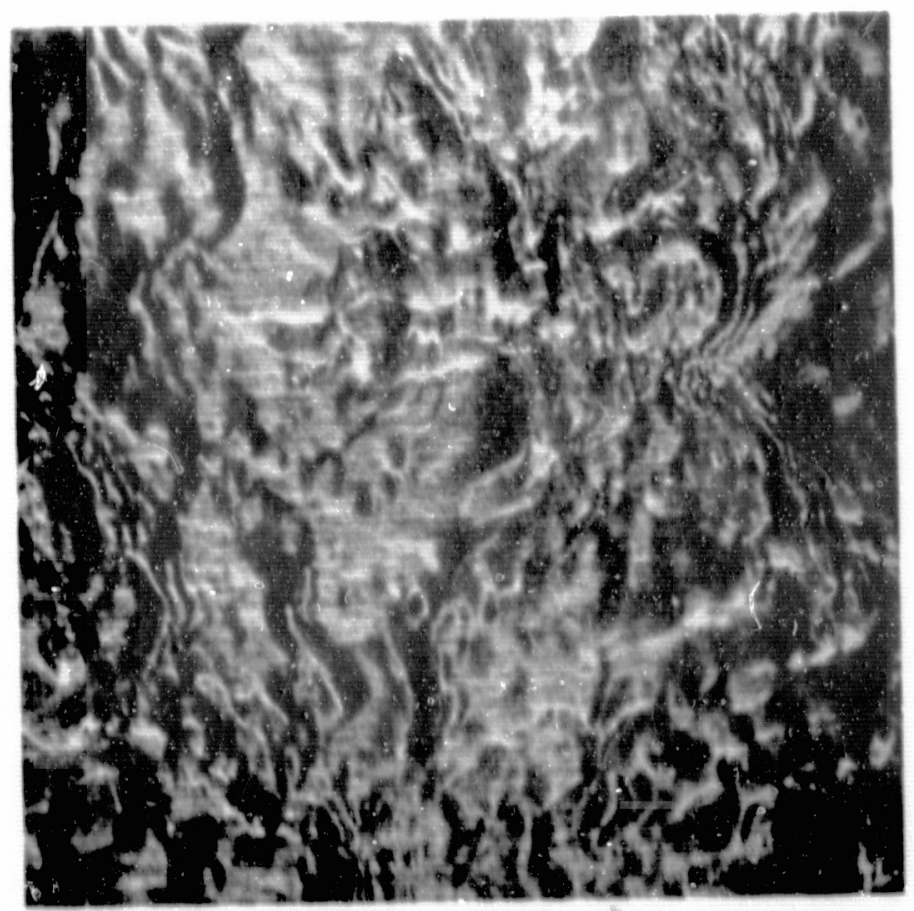

$$
\begin{aligned}
& T_{g}=42 \mathrm{~F} \\
& p=82 \mathrm{psia} \\
& u_{g}=35 \mathrm{fps} \\
& G=15.5 \mathrm{lb} / \mathrm{ft}^{2}-\mathrm{sec} \\
& M_{0}=17 \mathrm{lb} / \mathrm{ft}^{2}
\end{aligned}
$$

Methanol Film Coolant

(b) $m_{1}=0.08221 \mathrm{~b} / \mathrm{sec}-\mathrm{ft}$

Fig. 7 Influence of $m_{1}$ on Film Surface Characteristics-Low Gas Stream Velocity 
flow direction $\longrightarrow$

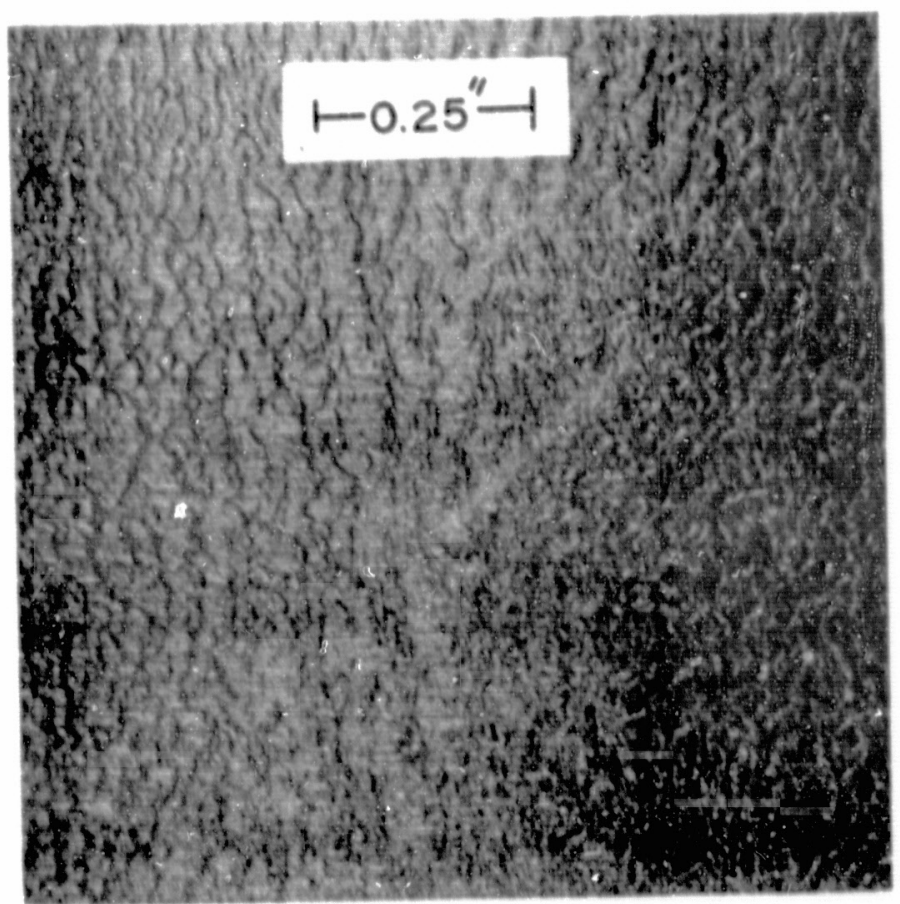

$T_{g}=38 \mathrm{~F}$

$p=77$ psia

$u_{\mathrm{g}}=115 \mathrm{fps}$

$G=50 \quad 1 \mathrm{~b} / \mathrm{ft}^{2}-\mathrm{sec}$

$M_{0}=178 \quad 1 b_{f} / f^{2}$

Methanol Film Coolant

(a) $m_{1}=0.0144 \quad 1 b / s e c-f t$

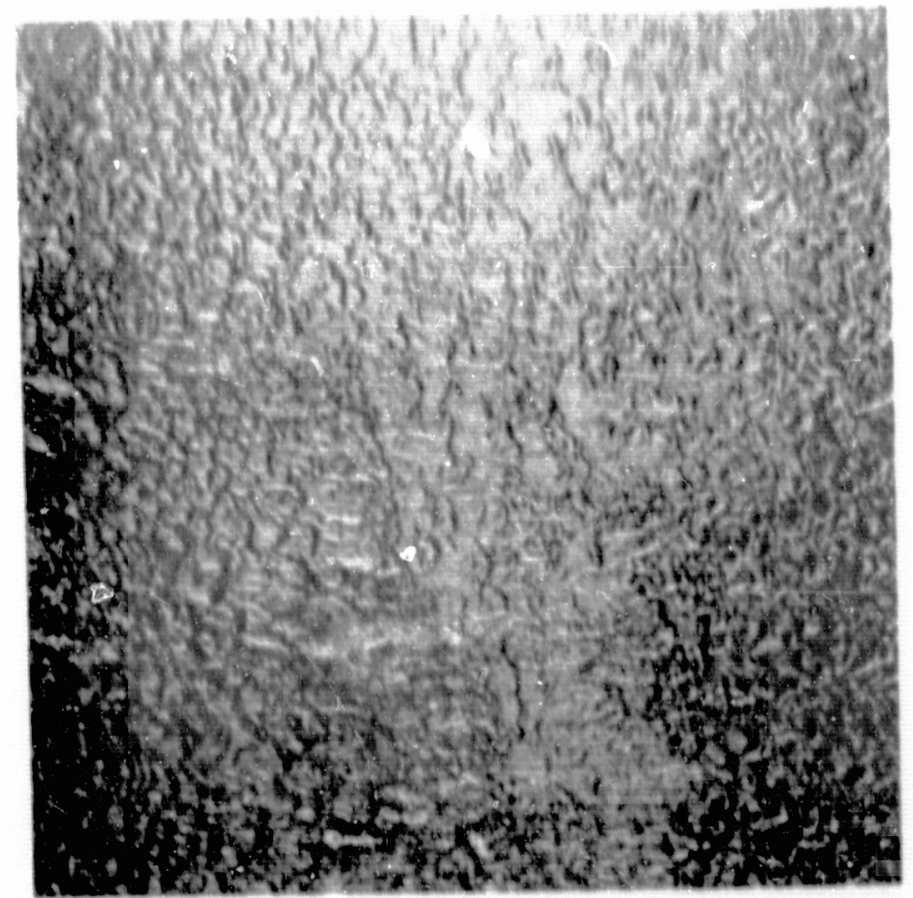

$\mathrm{T}_{\mathrm{g}}=38 \mathrm{~F}$

$p=77$ psia

$u_{g}=115 \mathrm{fps}$

$G=50 \mathrm{lb} / \mathrm{ft}^{2}-\mathrm{sec}$

$M_{0}=1781 b_{f} / f^{2}$

Methanol Film Coolant

(b) $m_{1}=0.08161 \mathrm{~b} / \mathrm{sec}-\mathrm{ft}$

Fig. 8 Influence of $m_{1}$ on Film Surface Characteristics-High. Gas Stream Velocity 
for low gas stream velocities, less than, say, $50 \mathrm{ft} / \mathrm{sec}$.

Figure 8 presents two photographs for the more impcrtant case where the gas stream velocity is relatively high (115 ft/sec in this case). Comparing these photographs with those shown in Fig. 7, it is seen that the size and the wave length of the disturbance waves on the surface of the liquic film has decreased significantly with the increase in the gas stream velocity. Moreover, comparison of Fig. 8 (a) and Fig. 8 (b). shows that the scale of the disturbance waves is not noticeably altered by increasing the rate of liquid injection $m_{1}$ from 0.0144 to $0.0316 \mathrm{lb} / \mathrm{sec}-\mathrm{ft}$. The study of a large numoer of such photographs indicated that, with the exception of those few cases that were investigated where the gas stream velocity was very low, the conclusion could be drawn that the basic scale of the small disturbancs waves on the surface of the liquid film was essentially independent of the rate of liquid injection.

Surface Tension $\sigma$. Figure 9 presents two photographs that were obtained under essentially the same flow conditions with two different ilquids. The liquid shown in Fig. 9 (a) is RP-l and the liquid shown in Fig. 9 (b) is water. The values of surface tension at the corresponding liquid temperatures in these photographs are approxiliaizly $19 \mathrm{dynes} / \mathrm{cm}$ for the RP-1 and 61 dynes/cni for the water. These photographs demonstrate that the scale of the interfacial disturbances is generally more ordered for the case where $B P-1$ is 
flow direction $\longrightarrow$

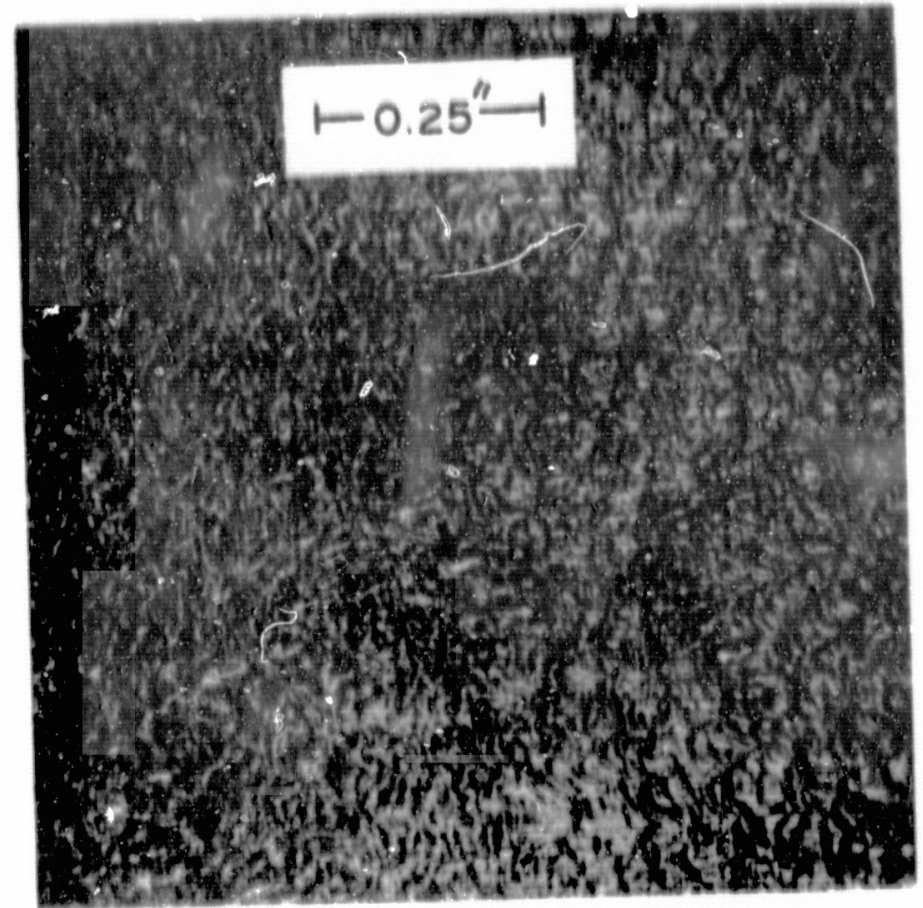

$R P-i f i 1 m$ coolant

$T_{g}=431 . F$

$p=152$ psia

$u_{g}=89 \mathrm{fps}$

$G=411 \mathrm{~b} / \mathrm{ft} \mathrm{t}^{2}-\mathrm{sec}$

$M_{0}=1121 b_{f} / f t^{2}$

$m_{1}=0.0361 \mathrm{~b} / \mathrm{sec}-\mathrm{ft}$

$\mu_{1}=2.4 \times 10^{-4} \quad 1 \mathrm{~b} / \mathrm{sec}-\mathrm{ft}$

(a) $\sigma=19$ dynes $/ \mathrm{cm}$

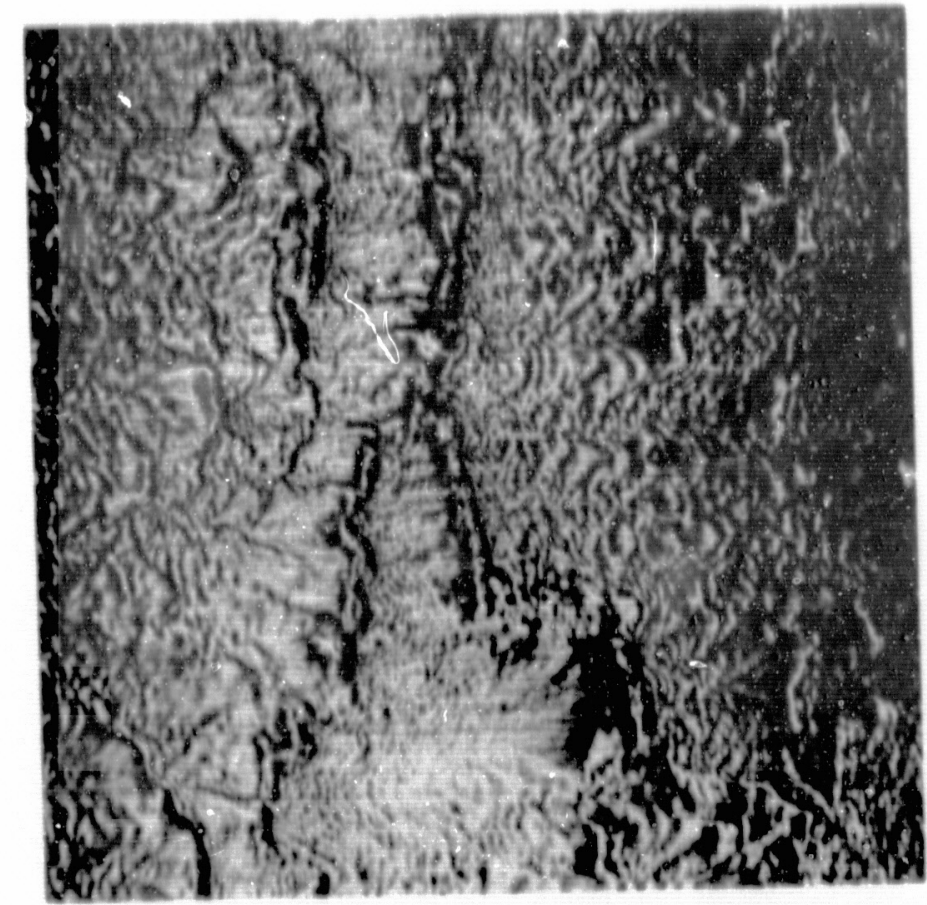

water film coolant

$T_{g}=495 \mathrm{~F}$

$p=148 \mathrm{psia}$

$u_{g}=95 \mathrm{fps}$

$G=421 \mathrm{~b} / \mathrm{ft}^{2}-\mathrm{sec}$

$M_{0}=1251 b_{f} / \mathrm{ft}^{2}$

$m_{1}=0.0581 \mathrm{~b} / \mathrm{sec}-\mathrm{ft}$

$\mu_{1}=2.1 \times 10^{-4} 1 \mathrm{~b} / \mathrm{sec}-\mathrm{ft}$

(b) $\sigma=61$ dynes $/ \mathrm{cm}$

Fig. 9 Influence of Surface Tension on Film Surface Characteristics 
the liquid. The surface of the water film is characterized by two types of disturbance waves, one having a larger scale and being more two-dimensional in appearance than the other. In fact, water was the only liquid that clearly showed two types of disturbance waves. The other liquids that were photographed, namely, methanol, butanol, and RP-l, did not exhibit that characteristic. Since the surface tension for water was signiricantly greater in all cases than the surface tension for the other 1 iquids investigated*, it is suggested that this phenomenon of the existence of two distinct types of disturbance waves for the water films was the result of the relatively high values of surface tension for that liquid.

Liquid Viscosity $\mu_{1}$. The photographs of the subject investigation did not permit a direct observation of the influence of the liquid viscosity on the surface characteristics of the liquid film. For any two photographic experiments for which the liquid viscosity differed substantially, there also occurred a substantial variation in one or more of the remaining pertinent flow parameters. However, by crudely interpolating between the results of several photographs, it was apparent that if the liquid viscosity had an influence on the surface characteristics for the liquid film, it was not pronounced. Relative to the surface tension for the liquid and to the pertinent gas stream parameters (which * See Fig. D.1 in Appendix 0 . 
are discussed in the following subsection), the liquid viscosity appeared to be of secondary importance in determining the structural characteristics of the gas-liquid interface.

\subsubsection{The Influence of the Gas Stream Parameters on the Film Surface Characteristics}

The present subsection discusses how the following parameters for the gas stream alter the surface characteristics of the liquid film: (a) the gas stream velocity $u_{g}$, (b) the gas mass velocity $G$ (defined by Eq. $(2-1)$ ), and (c) the momentum parameter $M_{0}(?$ sined by Eq. (2-2)). Because these three parameters are interrelated, it was not possible to conduct a series of experiments wherein any two of these parameters were held constant while the remaining parameter was varied. It was possible, however, to obtain photographic data where one of these parameters was held constant while the remaining two parameters were varied. It is thus possible, in an indirect way, to arrive at some conclusions about how the subject gas stream parameters influenced the film surface characteristics.

Constant $G$, Variable $u_{g}$ and $M_{0}$. Figure 10 presents two photographs of a liquid film where water is the liquid phase. The parameters listed in the figure show that the gas mass velocity $G$ is essentially the same for both photographs while the momentum parameter $M_{0}$ and the gas stream velocity $u_{g}$ vary substantially. Figure 10 shows that the scale of the interfacial disturbances decreases as the velocity $u_{g}$ (or the momentum parameter $M_{0}$ ) increases. It follows, therefore, 
flow direction $\rightarrow$

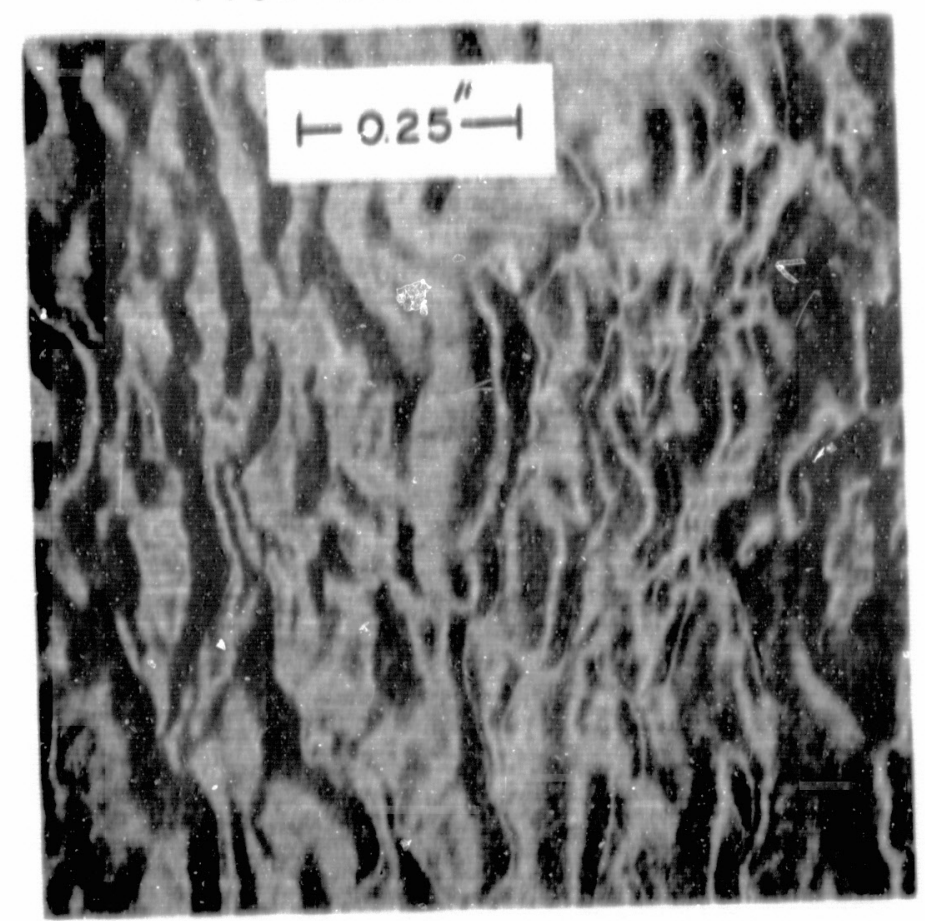

$T_{g}=20 \mathrm{~F}$

$p=78 \mathrm{ps} 4 \mathrm{a}$

$u_{g}=64 \mathrm{fps}$

$M_{0}=65 \quad 1 b_{f} / f t^{2}$

$m_{1}=0.03901 \mathrm{~b} / \mathrm{sec}-\mathrm{ft}$

Water Film Coolant

(a) $G=301 \mathrm{~b} / \mathrm{sec}-\mathrm{ft}^{2}$

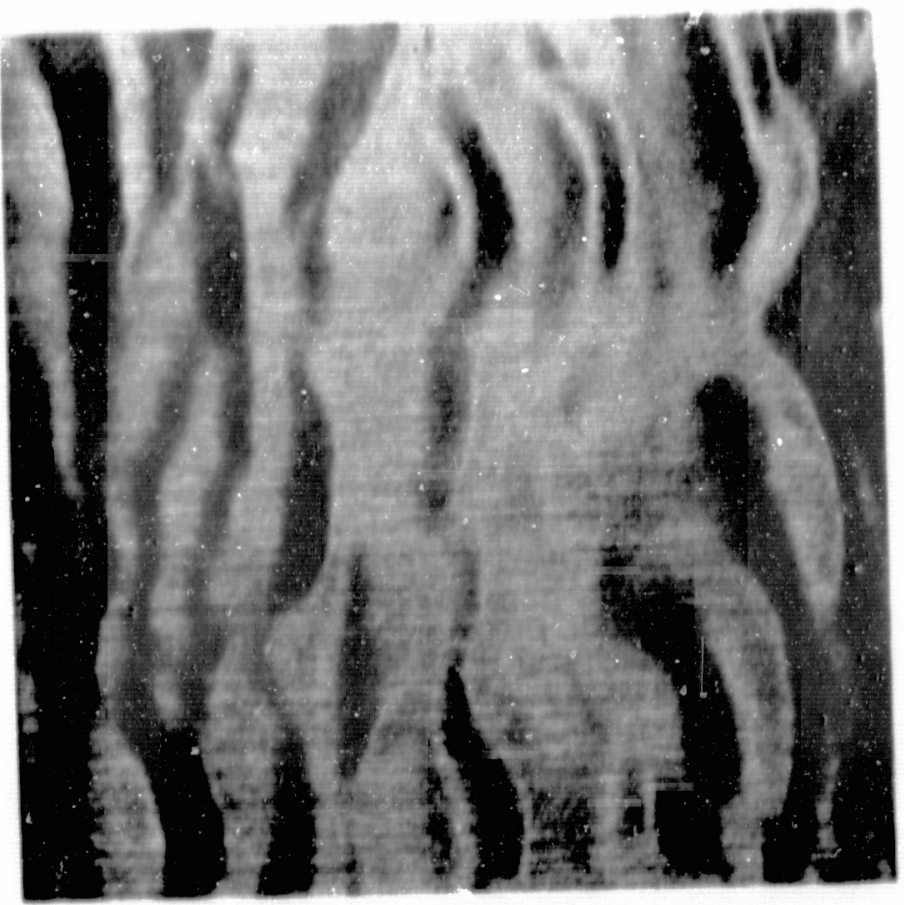

$T_{g}=20 \mathrm{~F}$

$p=150 \mathrm{psia}$

$u_{g}=31 \mathrm{fps}$

$M_{0}=26 \quad 1 b_{f} / f t^{2}$

$m_{1}=0.01621 \mathrm{~b} / \mathrm{sec}-\mathrm{ft}$

Water Film Coolant

(b) $G=281 \mathrm{~b} / \mathrm{sec}-\mathrm{ft} \mathrm{t}^{2}$

Fig. 10. Influence of $G, u_{g}$, and $M_{0}$ on $F i 1 m$ Surface Characteristics- $-G$ Constant 
that the value for the gas mass velocity $G$ (or, the gas stream Reynolds number) does not give sufficient information to characterize the scale of the interfacial waves.

Constant $u_{g}$, Variable $M_{0}$ and $G$. Figure 11 preserits two photographs of a iiquid film for which methanol is the liquid phase. The parameters listed in the figure show that the gas stream velocity $u_{g}$ is essentially the same for both photographs while the parameters $M_{0}$ and $G$ vary significantly. Figure 11 shows that the liquid film for which the gas mass velocity $G$ (and the momentum parameter $M_{0}$ ) has the largest value exhibits the smaller interfacial scale. It follows, therefore, that the value of the gas stream velocity $u_{g}$ does not give sufficient information to characterize the interfacial film structure.

Constant $M_{0}$, Variable $u_{g}$ and $G$. Figure 12 presents two photographs for a methanol liquid film for which the momentum parameter $M_{0}$ has essentially the same value while the gas stream velocity $u_{g}$ and gas mass velocity $G$ vary substantially. The scale of the interfacial wave pattern in each photograph is virtually the same. The photographic data suggest, therefore, that the momentum parameter $M_{0}$ is the variable for the gas stream that most governs the resultant film surface characteristics. This observation has not been made previously in the published literature, due in part to the fact that very few (if any) detailed investigations into film surface characteristics have been made for 


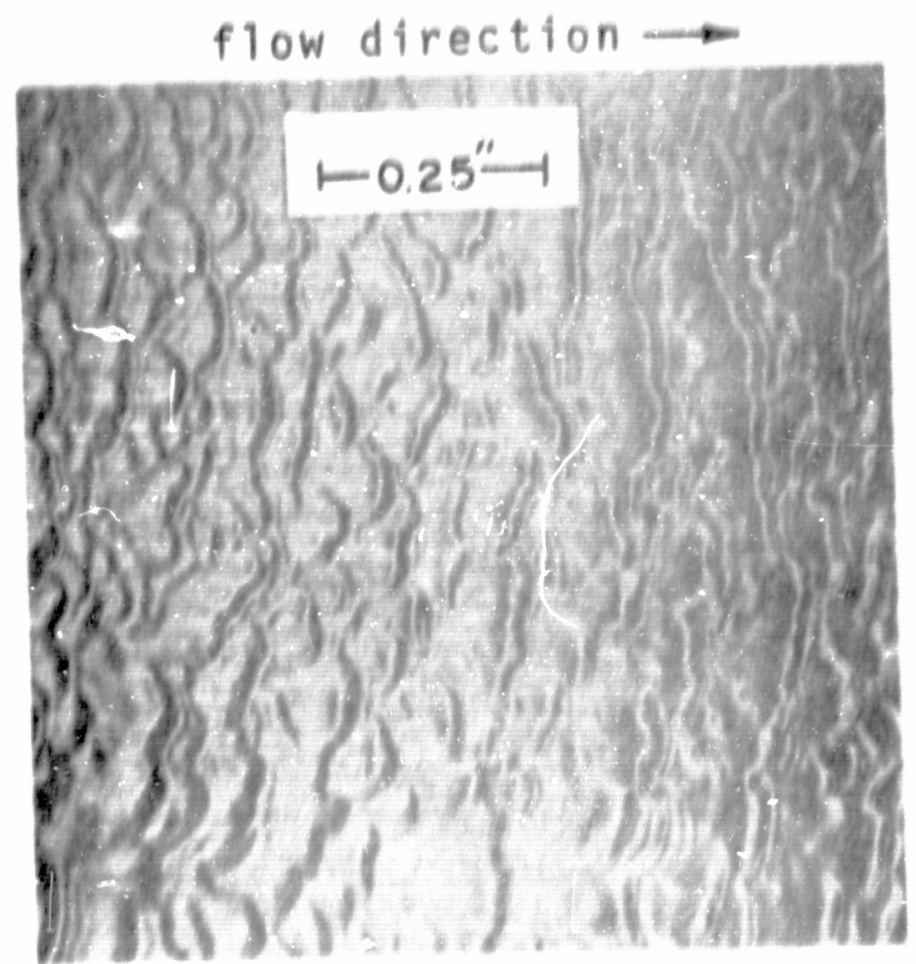

$T_{g}=30 \mathrm{~F}$

$p=79 \mathrm{psia}$

$G=251 \mathrm{~b} / \mathrm{ft} \mathrm{t}^{2}-\mathrm{sec}$

$M_{0}=221 b_{f} / f t^{2}$

$m_{1}=0.01381 \mathrm{~b} / \mathrm{sec}-\mathrm{ft}$

Methanol Film Coolant

(a) $u_{g}=58 \mathrm{fps}$

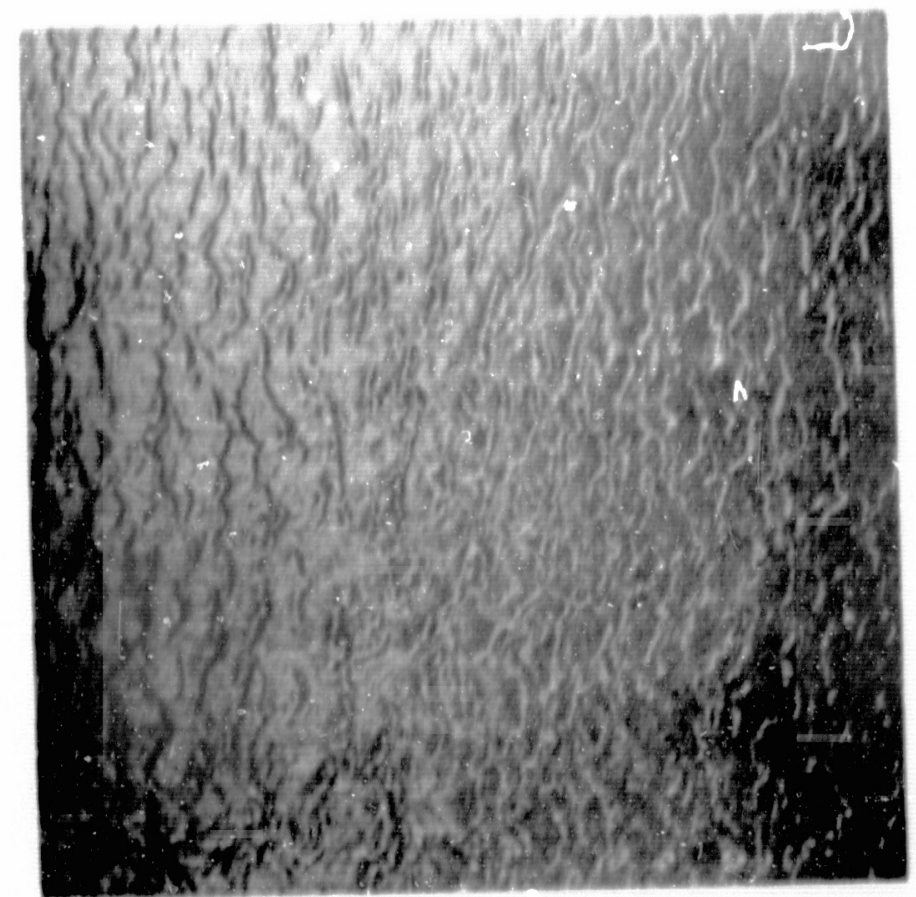

$$
\begin{aligned}
& T_{g}=30 \mathrm{~F} \\
& P=155 \mathrm{psia} \\
& G=561 \mathrm{~b} / \mathrm{ft}^{2}-\mathrm{sec} \\
& M_{0}=1121 \mathrm{~b}_{\mathrm{f}} / \mathrm{ft}^{2} \\
& m_{1}=0.01381 \mathrm{~b} / \mathrm{sec}-\mathrm{ft} \\
& \text { Methanol } \mathrm{Film} \mathrm{Coolant}
\end{aligned}
$$

(b) $u_{g}=66$ fps

Fig. 11. Influence of $G, u_{g}$, and $M_{0}$ on Film Surface Characteristics $=-u_{g}$ Constant 
flow direction $\rightarrow$

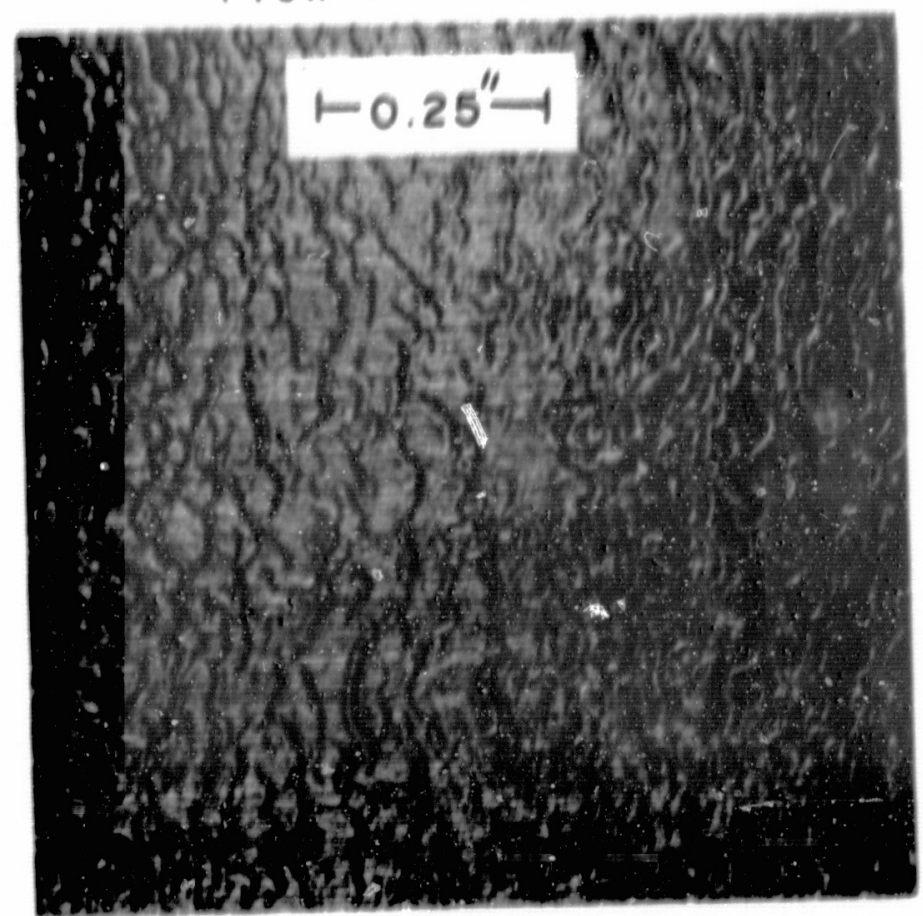

$T_{g}=40 \mathrm{~F}$

$p=80$ psia

$u_{g}=90 \mathrm{fps}$

$G=391 \mathrm{~b} / \mathrm{ft} \mathrm{t}^{2}-\mathrm{sec}$

$m_{1}=0.01441 \mathrm{~b} / \mathrm{sec}-\mathrm{ft}$

Methanol $\mathrm{F} 11 \mathrm{~m} \mathrm{Coolant}$

(a) $M_{0}=1081 b_{f} / f t^{2}$

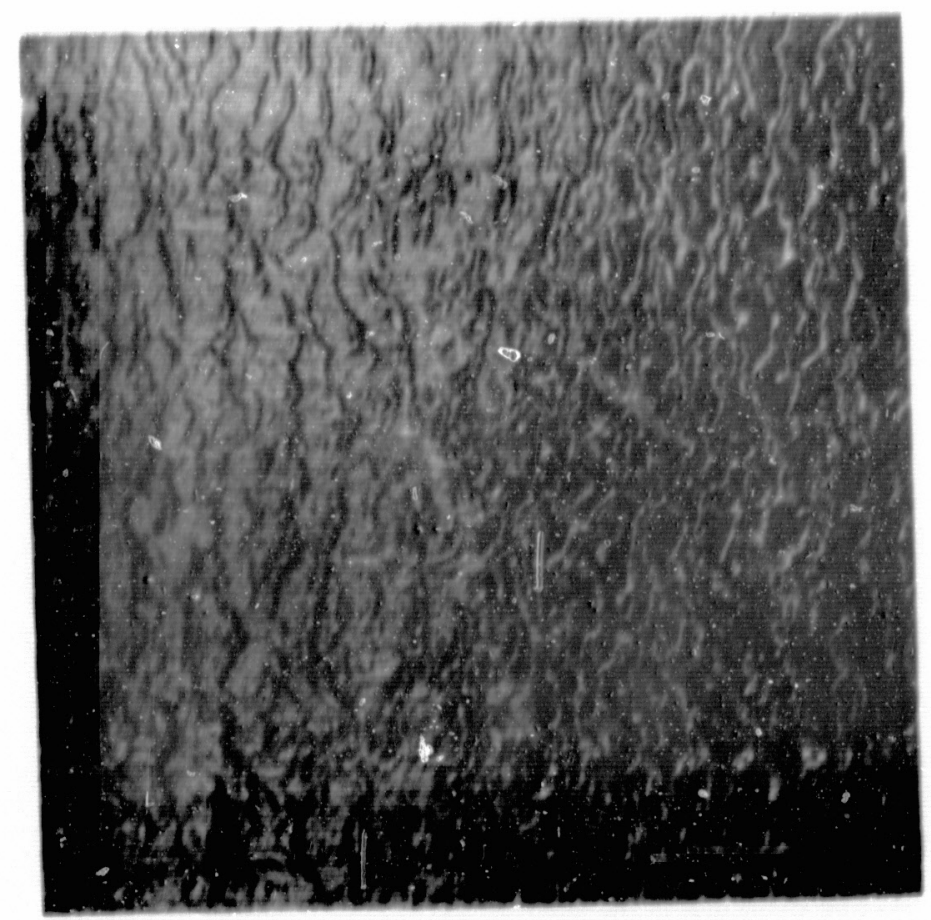

$T_{g}=30 \mathrm{~F}$

$p=155$ psia

$u_{g}=66 \mathrm{fps}$

$G=56 \mathrm{lb} / \mathrm{sec}-\mathrm{ft}^{2}$

$m_{1}=0.01381 \mathrm{~b} / \mathrm{sec}-\mathrm{ft}$

Methanol Film Coolant

(b) $M_{0}=1121 b_{f} / f t^{2}$

Fig. 12. Influence of $G, u_{g}$, and $M_{0}$ on Film surface
Characteristics-- $M_{0}$ Constant 
gas stream pressures and temperatures significantly different from ambient. That fact has precluded the independent investigation of the influence of each of the parameters $u_{g}, M_{0}$, and $G$ on the characteristics of the surface of the liquid film. (That is, for a constant value of the gas stream density, it is not possible to hold any two of the parameters $u_{g}, G$ and $M_{0}$ constant while the remaining parameter is varied.) The foregoing observation, however, is related in a fundamental way to the correlation for the mass transfer data that is developed in Section 3.1 of this report.

\section{4 .3 Summary.}

The observations made in Subsection 2.4 .1 and in Subsection 2.4 .2 regarding the influence of the pertinent liquid and gas stream variables on the character of the surface of a liquid film can be summarized as follows:

(a) The interfacial structure is not noticeably altered by substantial variations in the rate of liquid injection $m_{1}$, except for those cases where the gas stream velocity is very low (less than approximately $50 \mathrm{ft} / \mathrm{sec}$ ).

(b) The liquid viscosity $\mu_{1}$ apparently does not influence the surface characteristics to any significant degree. (c) The surface tension o apparently alters the surface niucacteristics in that two distinct wave forms ware gener ally observed when water was the liquid phase (relatively high values for $\sigma$ ) that were not observed for either butanol, methanol, or RP-l as the liquid phase (relatively low values 
for $\sigma)$.

(d) The scale of the small interfacial disturbances is well characterized by the value for the momentum parameter $M_{0}$ (and not $G$ or $u_{g}$ ) for the gas stream; the scale of the interfacial disturbances decreases as the value for the momentum parameter $M_{0}$ increases.

\subsection{The Data for Mass Transfer for a Constant Velocity Gas Stream}

The following procedure was employed to obtain experimental data on the rate of mass transfer from the liquid film to the gas stream. The hot gas generator was started and preselected values for the gas stream temperature, pressure, and air flow rate (gas stream Reynolds number or gas mass velocity) were established. The liquid film flow rate was established and the electric heat exchanger for preheating the liquid was turned on. Sufficient time was allowed to attain thermal equilibrium (normally 10 minutes) bcfore the mass transfer measurements were made.

Prior to making quantitative mass transfer measurements it was necessary to insure that the liquid film was properly wetting the test surface and that the capture slot was functioning so as to remove all the liquid remaining on the test surface. First a visual observation was made through the Pyrex window in the roof of the test section to determine the wetting characteristics of the liquid film. During the course of the investigation it was found that if any 
non-uniformities existed in the fiow field, the liquis film would not remain in the trough that was machined into the test plate. Frequentiy one of the mixing screens muld fail (due to the high gas stream temperature and the large pressure drop across the screen), resulting in an irregular gas flow field and a corresponding poorly wetted tesi plate The only experimental data that are renorted herein are those for which the liquid film remained in the trough and completely wetted the $2-i n$. by $10-i n$. test surface.

Since the rate of mass transfer from the liquid film was determined as the difference between the rate of liquid injection and the rate of liquid withdrawal, the necessity of an accurate determination of these flow rates is ajparent. The rate of liquid injection was determined by means of a turbine flowmeter. The flowmeter was normally calibrated before each experimental test by the time-weight technique. During the course of an experimental test, the output of the turbine flowmeter was continuously recorded on a stripchart recorder. A cyclone separator was employed to measure. ie rate at which liquid was withdrawn from the test plate at the capture slot*. As mentioned previously, the effective operation of the capture sict required that a certain amount of the gas flow be withdrawn with the liquid, thus producing a two-phase withdrawal flow. The gas and liquid phases were separated by means of the cyclone separator, thereby

* See Appendix 8.4 for the design of the separator. 
enabling the measurement of the liquid withdrawal rate. The cyclone separator was found to provide effective separation of the gas and liquid phases. In preliminary experimentation, it was found that under conditions of negligible mass transfer from the liquid film (cold, low velocity gas flow) the rates of liquid injection and vithdrawal generally agreed to within less than 5 percent. Even with the effective performance of the cyclone separator, it was recognized that the direct contact of the gas and liquid phases during the flow through the separator could result in some error in the measurement of the rate of liquid withdrawal due to saturation of the gas flow with liquid vapor. Thus, it was desired to keep the rate of gas flow through the separator as low as possible while maintaining effective operation of the capture siot. The procedure employed is described below. Once it was ascertained that the wetting characteristics of the film were correct, the liyuid flow rate was set at the maximum value to be investigated. The valve on the separator which controlled the rate at which the gas phase was vented to the surroundings (and thus controlled the rate at which gas was withdrawn at the capture slot) was opened a small amount. A measurement was made of the rate at which the liquid was being captured at the withdrawal slot. The vent valve was then opened further and a second measurement of the rate of liquid withdrawal was made. This procedure was repeated until a further opening of the vent 
valve did not increase the rate of liquid withdrawal.

Figure 13 presents a typical plot of the rate of liquid withdrawal as a function of the rate at which air was vented from the cyclore separator. The figure illustrates that the rate of liquid withdrawal increases rapidly at first with an increasing vent flow rate and then reaches a maximum, or plateau, such that a further increase in the vent flow rate does not appreciably affect the rate of liquid withdrawal. The increase in $m_{2}$ with vent gas flow rate reflects an increase in effectiveness of the capture slot in withdrawing the liquid from the test surface. When the vented qas flowrate is too low, some of the liquid spills over the capture slot and then $m_{2}$ is not the total film flowrate at the point of withdrawal. As the vented gas flowrate is increased, the spillage is reduced until with sufficient vent flow the capture slot collects and withdraws all of the liquid on the test surface. In all of the experimental tests, the vent flow rate was always set equal to or greater than the value required to realize no change in the rate of liquid withdrawal with an increase in the vent flow rate. For the magnitude of the vent flow rate normally required, rough calculations showed that even if the gas phase that entered the separator was completely dry air, and the gas phase that was vented from the separator was completely saturated with vapor at the corresponding pressure within the separator, the amount of mass transfer that would have occurred 


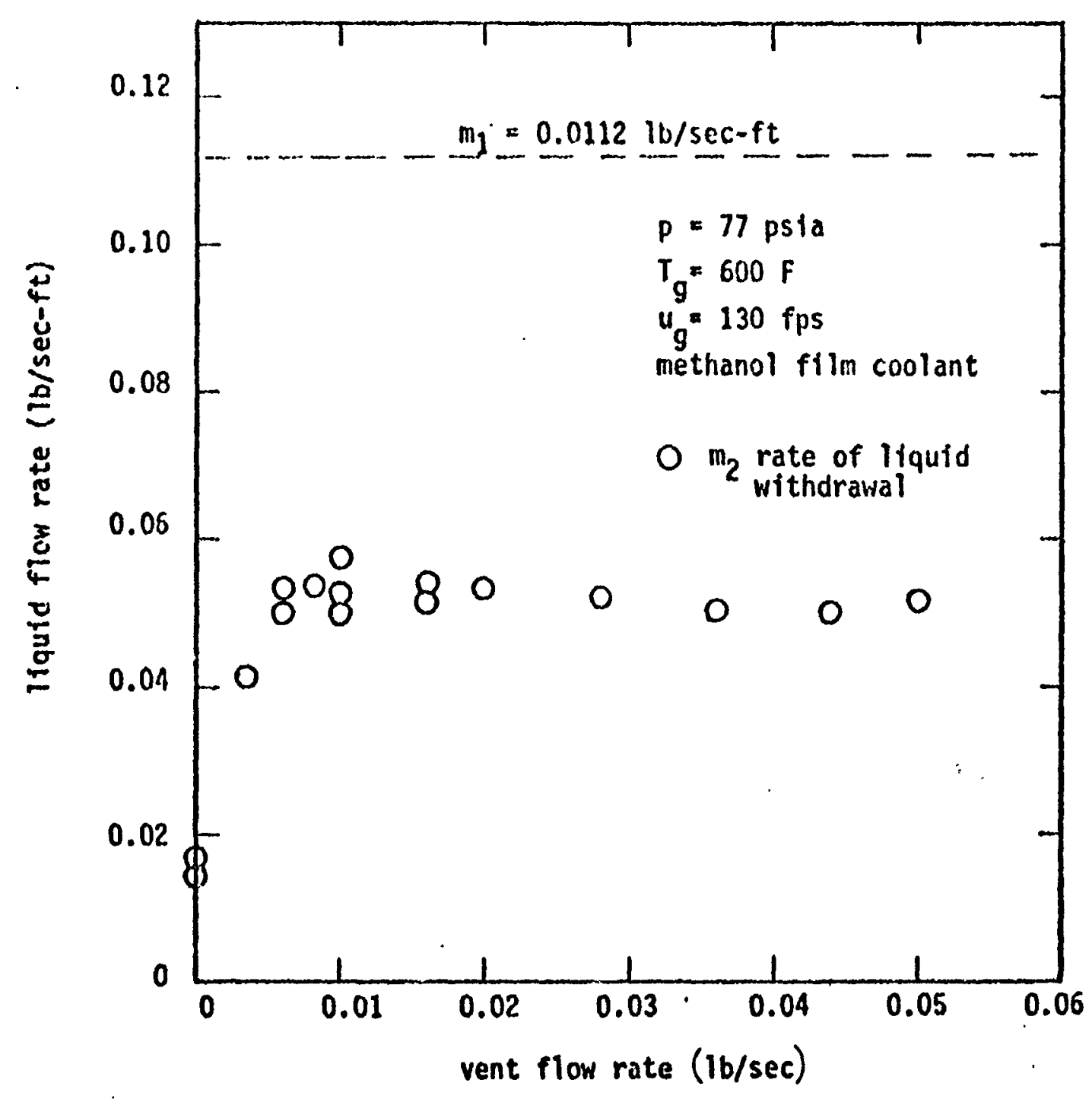

Fig. 13. Rate of Liquid Withdrawal as a Function of Vent Flow Rate for Separator 
within the separator would be negligible in comparison to the total rate of mass transfer that was normally realized from the iqquid film to the gas strean (not more than 5 percent).

After the velit flow rate was set, the quantitative mass transfer data were obtained by decreasing the rate of liquid injection in incremental stages and making the corresponding measurements of the rate of liquid withdrawal. Thus, during any one experimental test, the only parameter that was varied was the rate of liquid injection.

Figure 14 illustrates the typical nature of the experimental data for mass transfer that were obtained in the present investigation. The data shown in Fig. 14 are for methanol as the liquid phase and they are for a nominal gas stream temperature and pressure of $400 \mathrm{~F}$ and $75 \mathrm{psia}$, respectively. Plotted in the figure is the rate of liquid withdrawal, $m_{2}$, as a function of the rate of liquid injection, $m_{1}$, with the gas stream .elocity, $u_{g}$, as a parameter. The solid curve shown at an inclination of $45^{\circ}$ represents the condition of no mass transfer; at any point on that curve, the rate of liquid injection is equal to the rate of liquid withdrawal. The vertical distance between the solid curve and any one datum point represents the total rate of mass transfer from the liquid film for that particular combination of flow conditions. Figure 14 illustrates the important fact that the rate of mass transfer from the 


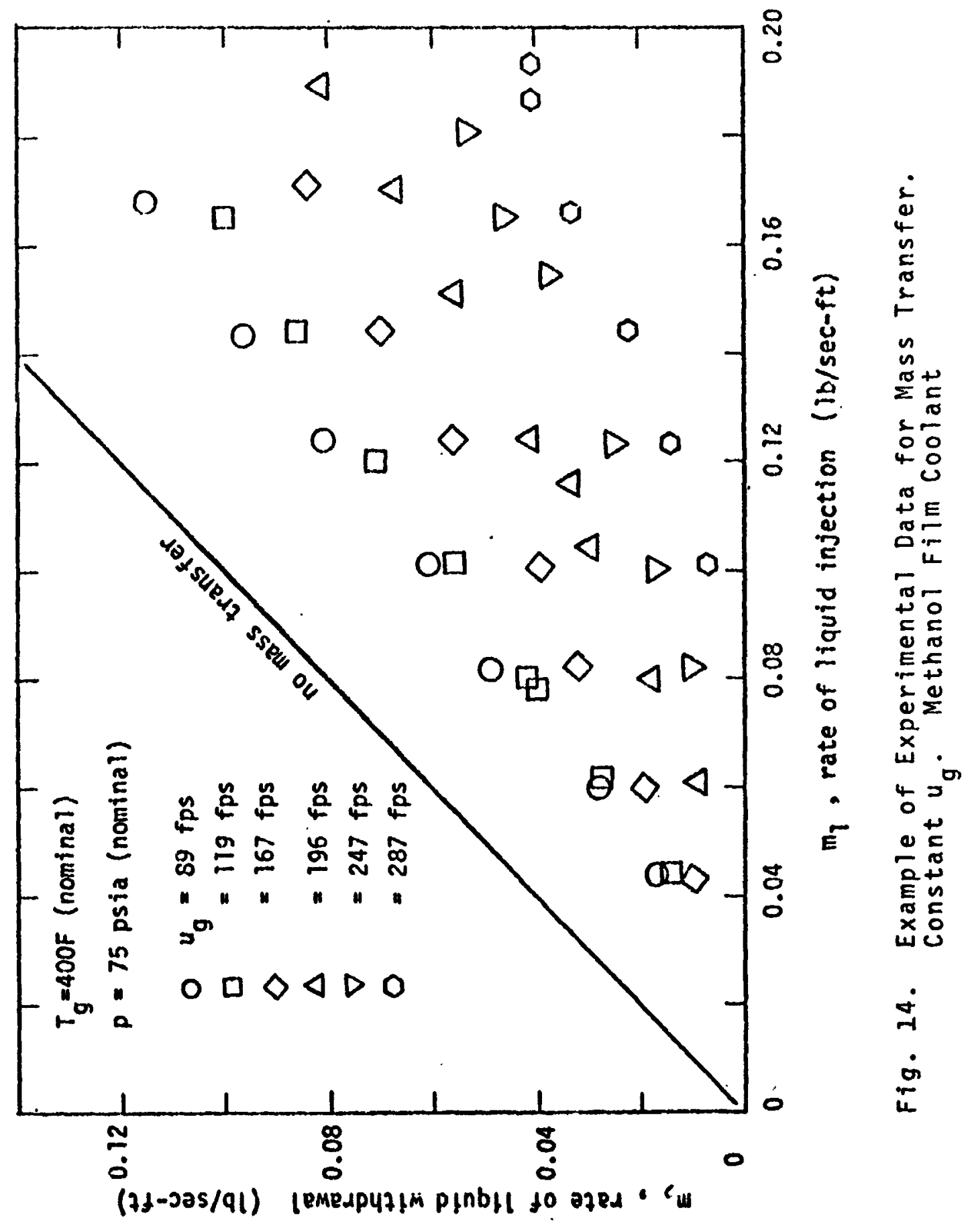


liquid film to the gas stream was found to be a function of the rate at which liquid was injected onto the test plate; the theory for simple mass transfer, however, does not predict any such dependence on the rate of liquid injection. Figure 14 shows further that, to within the accuracy of the experimental data, the total rate of mass transfer from the liquid film to the gas stream increased linearly with the rate of liquid injection. This fact was not foreseen before the experimental investigation was begun, but it greatly simplified the correlation of the experimental data, as is discussed in detail in Section 3.1 of this report.

The remainder of this section discusses how the pertinent flow parameters for both the liquid and gas phases influenced the net rate of mass transfer from the liquid film to the gas stream. The mass transfer data of some of the experimental tests is considered in detail. Although it was not feasible to discuss the data of each experimental test of the present investigation, it should be noted that the illustrative cases that are considered are representative of all of the tests of the present investigation. That includes not only those tests for which a constant velocity, hot gas stream was employed, but also those tests where the hot gas stream was accelerated over the wetted test plate and those tests for which the gas flow was not heated (cold flow, constant velocity gas stream). An inclusive graphical presentation of the data for mass transfer is given in 
Appendix G.

Subsection 2.5.1, which follows below, discusses the influerce of the liquid surface tension and the liquid viscosity on the total rate of mass transfer. Subsection 2.5 .2 discusses the influence of the gas stream flow parameters on the measured rate of mass transfer from the liquid film to the gas stream.

2.5.1 The Influence of the Surface Tension and the Liquid Viscosity on the Data for Mass Transfer

Figure 15 serves to illustrate the influence of both the surface tension $\sigma$ and the liquid viscosity $\mu$ upon the experimental data for mass transfer. Presented in the figure are the experimental data for mass transfer that were obtained for the experimental tests 113, 205, and 37. The liquids employed in these three tests were methanol, butanol, and water, respectively. The pertinent flow parameters for each test are listed in the figure. The dashed curves presented in Fig. 15 represent the condition of simple mass transfer as computed for the pertinent gas flow conditions*. If there had been no entrainment of liquid into the gas stream and if the liquid film had presented a smooth boundary to the developing boundary layer, the experimental data would lie along the dashed curves. Thus, the vertical distance between any one of the dashed curves and the datum

* Subsection 3.1.2 discusses how the simple mass transfer rates were evaluated. Table G.I of Appendix $G$ lists the average value for the simple mass transfer rate for each experimental test that is reported. 


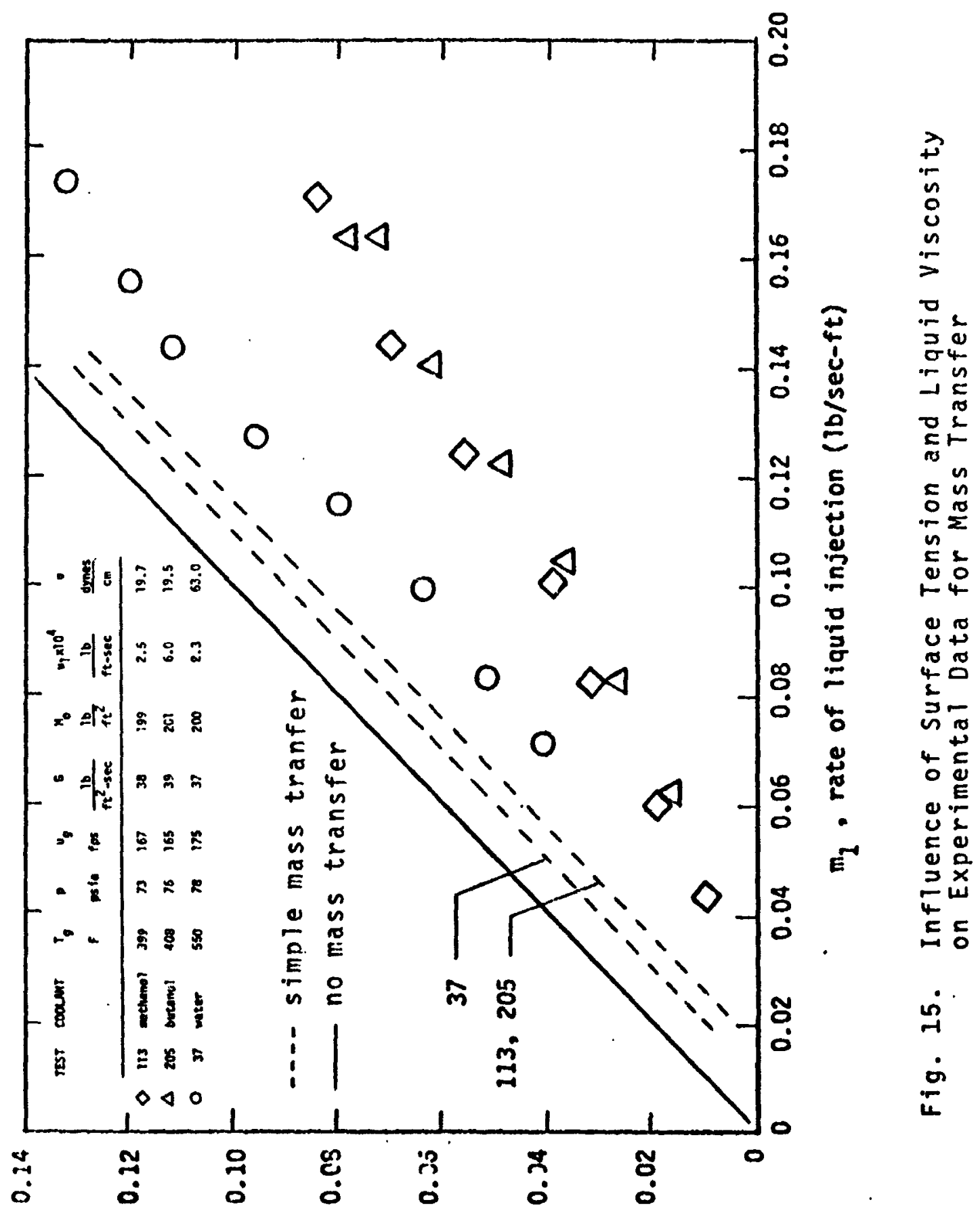

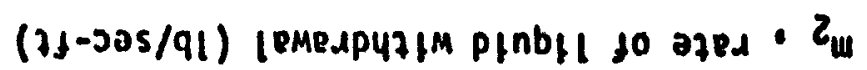


points for tnat curve represents the contribution to the total rate of mass transfer that is due to the interfacial phenomena (film surface roughness and entrainment).

The three cases presented in Fig. 15 were chosen for purposes of illustration because the pertinent flow parameters for the gas stream, with the exception of the gas stream temperature, $T_{g}$, are essentially the same while the viscosity and surface tension for the liquid phase are significantly different*.

Compare first the data that were obtained for water (test 37 ) and the data that were obtained for methanol (test 113). Figure 15 shows that the mass transfer due to the interfacial phenomena is much greater for the case where. methanol is the liquid phase. Referring to the values for the pertinent parameters listed in Fig. 15, it is seen that only the surface tension $\sigma$ is significantly different for these two cases. These data suggest, therefore, that the surface tension influences to a considerable degree the rate of mass transfer from a thin liquid film to a high velocity gas stream.

Comparing the data for methanol (test 113) and the data for butanol (test 205) in Fig. 15, we note that the measured rates of mass transfer are essentially the same for both cases. Moreover, the simple mass transfer contribution is

*The temperature employed to evaluate the liquid viscosity $\mu_{1}$ and the surface tension $\sigma$ was the average of the liquid temperature at the point of liquid injection and at the point of liquid withdrawal. 
virtually the same for each case so that the contribution due to interfacial phenomena is the same. In comparing these two cases, it is important to note that all of the parameters listed in the figure are nearly the same, except for the liquid viscosity $\mu_{j}$. The liquid viscosity for butanol for test 205 is roughly 2.5 times that for mathanol for test 113. These data suggest, therefore, that the liquid viscosity has no appreciable influence on the rate of mass transfer from a liquid film to a high velocity gas stream. The significance of that result was previously commented on in section 2.4. It was pointed out that a number of investigators have proposed that a liquid-film Reynolds number (which is inversely proportional to the liquid viscosity $\mu_{1}$ ) should be of primary importance in determining the rate at which liquid is entrained from the surface of a thin liquid film by a high velocity gas stream. The results of the present investigation, however, do not support that proposal.

It should be emphasized that the aforementioned dependence of the net mass transfer rate on the surface tension $\sigma$ was exhibited by all of the experimental data of the present investigation, including those obtained under cold-flow conditions, and those realized for the case where the gas stream was accelerated over the wetted test plate. 
2.5.2 The Influence of the Gas Stream Parameters on the Data for Mass Transfer

Figure 16 serves to illustrate how the pertinent parameters for the gas stream $\left(u_{g}, G\right.$, and $\left.M_{0}\right)$ influenced the experimental data for mass transfer. The figure presents the data for mass transfer that were obtained for the experimental tests 210,205 , and 213. The flow conditions for each test are indicated in the figure. The liquid phase employed in each test was butanol. The dashed lines in Fig. 16 indicate the condition of simple mass transfer.

Compare first the experimental tests 210 and 205. Figure 16 shows that the total rate of mass transfer for test 205 was significantly greater than for test 210 . The contribution due to simple mass transfer, hovever, was essentially the same for both cases. It follows, therefore, that the mass transfer due to the interiacial phenomena was greater for test 205 than it was for test 210 . The parameters listed in Fig. 16 indicate that the gas stream velocity, $u_{g}$, and the momentum parameter for the gas stream, $M_{0}$ " differed substantially for the two cases, while the gas mass velocity, G, was essentially the same for each case. Consider next the data shown in Fig. 16 for test 210 and for test 213. The parameters 1isted in the figure indicate that the momentum parameter $M_{0}$ was the same for both cases, while the remailing parameters show a varying degree of difference. The realized rates of mass transfer for the 


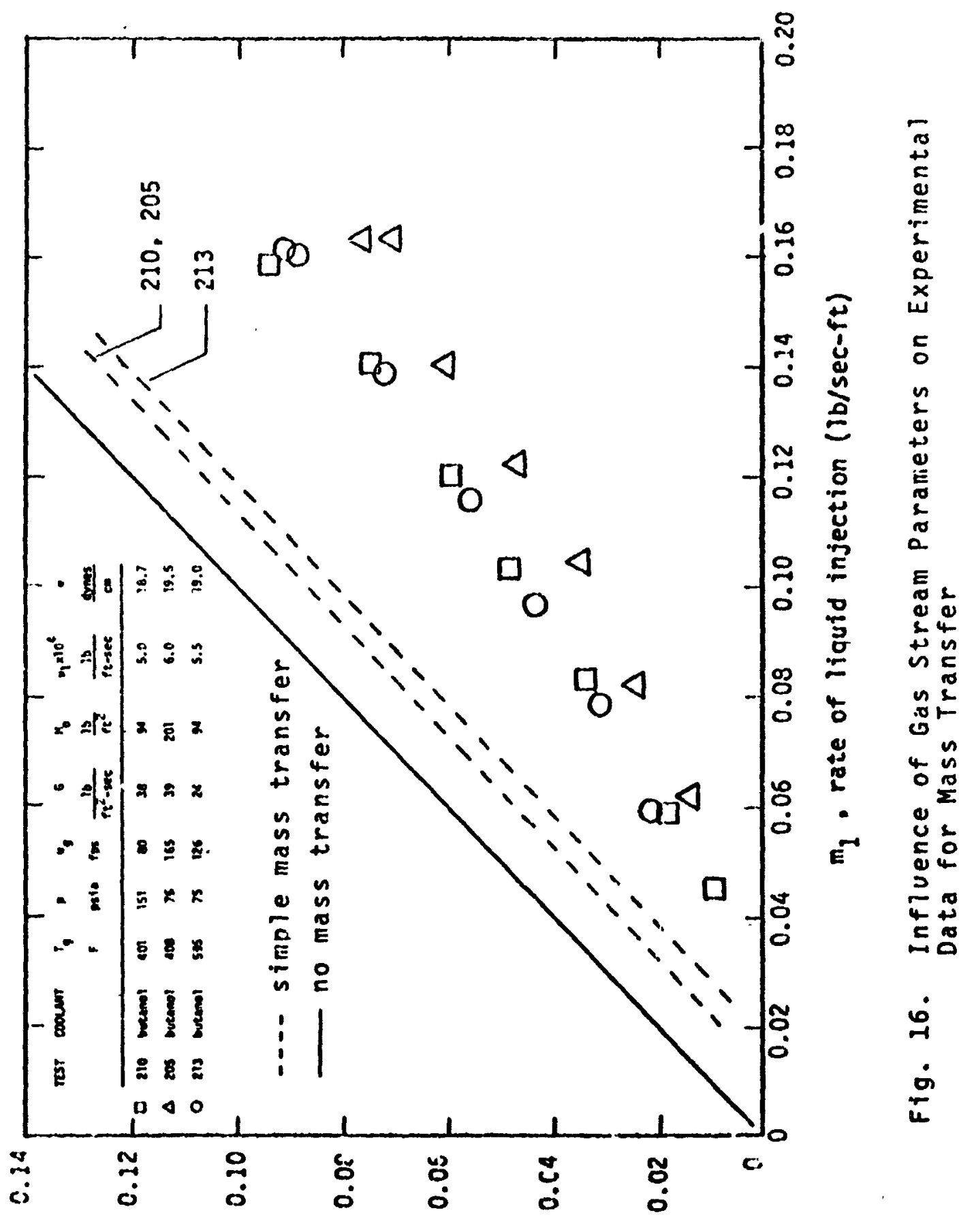

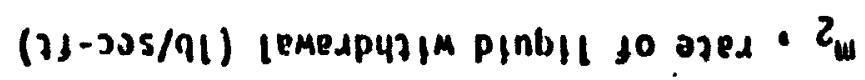


two cases are essentially the same. The data of Fig. 16 suggest, therefore, that the momentum parameter $M_{0}$ for the gas stream characterizes, to a large degree, the extent of the contribution to the net rate of mass transfer that is due to the interfacial phenomena. Again it should be emphasized that this observation was supported by all of the experimental data of the present investigation, and not by just those that are presented in Fig. 16.

\subsubsection{Summary}

The foregoing discussion suggests that the contribution to the net rate of mass transfer that is due to the interfacial phenomena:

(a) increases in a linear manner with the rate of liquid injection, $m_{1}$;

(b) increases with decreasing values for the surface tension $\sigma$;

(c) is apparently not a function of the viscosity for the liquid, $\mu_{j}$; and

(d) is characterized by the momentum parameter for the gas stream, $M_{0}$, with the rate of mass transfer increasing with increasing values for $M_{0}$.

These observations form the basis for the mass transfer correlation that is cieveloped in section s.t ur this report. 


\subsection{The Data for llass Transfer for an}

The stagnation temperature for the gas stream vas maintained constant at $400 \mathrm{~F}$ for that phase of the investigation where the gas stream was accelerated over the wetted test surface. Moreover, the Mach number profile was fixed, such that the Mach number increased from 0.14 at the point of liquid injection to 0.51 at the point of liquid withdrawa?*. The stagnation pressure was varied from 50 to 150 psia in increments of $25 \mathrm{psia}$, and both water and methanol were employed as the liquid phase. The experimental procedure employed in these investigations was basically the same as that outlined in the previous section.

Figure 17 presents all of the experimental data that was obtained in this portion of the investigation. Plotted in the figure is the rate of liquid withdrawal as a function of the rate of liquid injection with the stannation pressure, $p^{0}$, as a parameter. The experimental data obtained for both water and methanol as the liquid phase are shown in Fig. 17. Again, like the data that was presented in Figs. 14, 15, and 16: the total rate of mass transfer (the vertical distance between the line for no mass transfer and any one datum point) increases linearly with the rate of liquid injection. The data in Fig. 17 demonstrate that for the same flow conditions (same total pressure, total temperature, and degree

*i.e., only one area-contraction ratio (one aluminum wedge in Fig. 5) was emnloyed in the investigation 


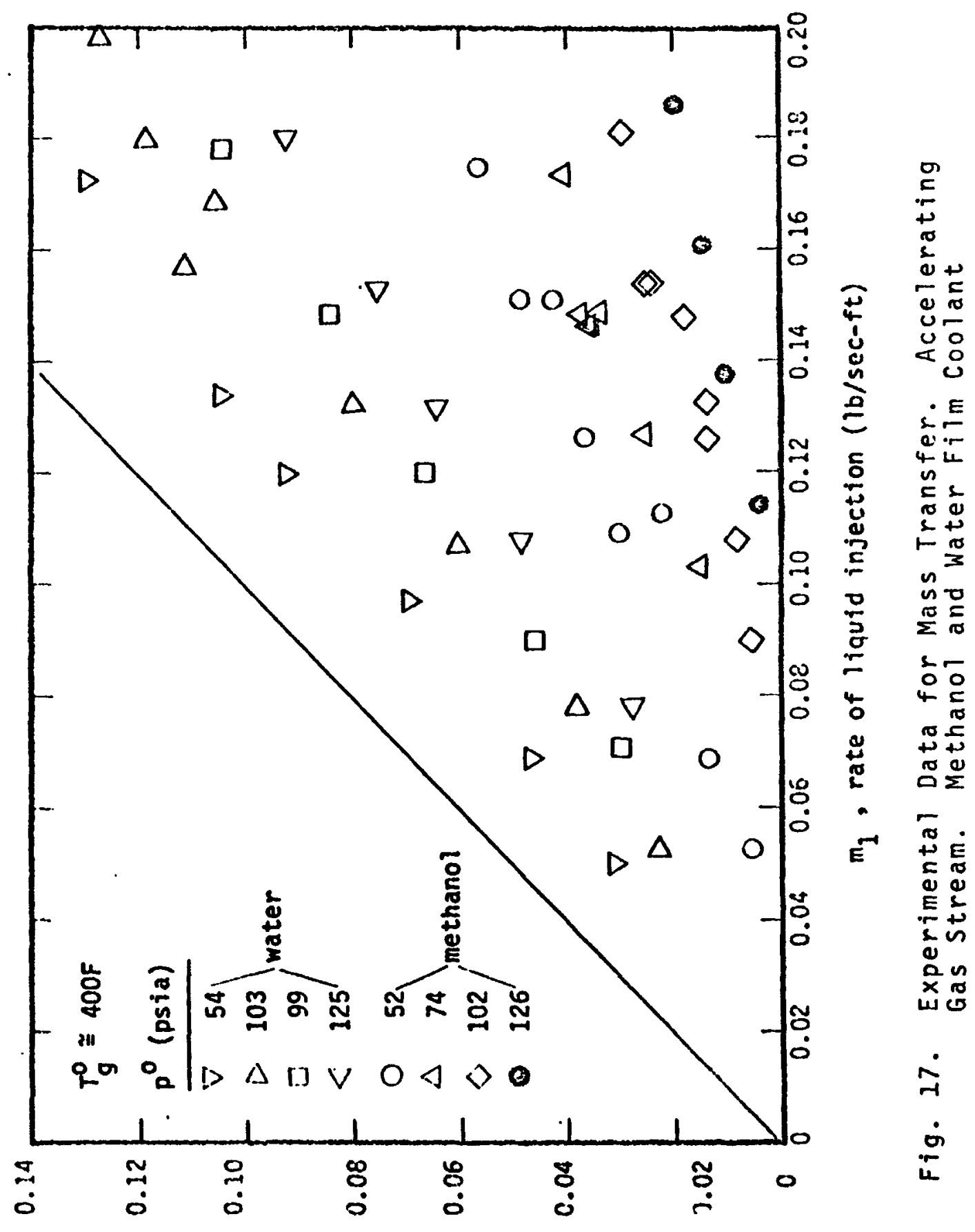

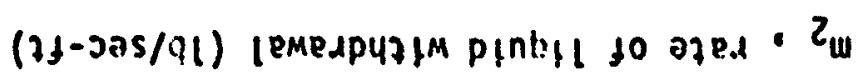


of acceleration of the gas streaml) the rate of mass transfer was much greater for the case where methanol was the liquid phase than for the case where water was the liquid phase. That is due, in part, to the fact that the heat of vaporization for waier is roughly 3 times that for methanol, so that for a given rate of heat transfer to the surface of the liquid film, the rate of vaporization of methanol is greater than is the rate of vaporization of water. But, more importantly, the rate of entrainment of methanol into the gas stream was much greater than was the rate of entrainment of water because the surface tension for methanol is roughly one-third that of water. The rather strong dependence of the rate of mass transfer from the liquid film into the gas stream on the surface tension was discussed in subsection 2.5.1 of the present report.

A correlation is developed in Section 3.3 for the mass transfer data presented in Fig. 17. It will be shown that the data correlation is a natural extension of the correlation that is developed in section 3.1 for the experimental data for mass transfer for the case where the gas stream velocity was essentially constant over the length of the wetted test plate. 


\subsection{The Data for the Maximum Liquid \\ Film Temperature}

In general, $T_{1}$, the temperature of the liquid at any point within the liquid $f i l m$, is less than $T_{s}$, the tempera: ture at the surface of the liquid film, and is greater than $\mathrm{T}_{W}$, the temperature at the surface of the wetted wall. However, for the special case where the wetted wall is adiabatic (as was the case for the present investigation), it is possible to obtain the condition

$$
T_{s}=T_{1}=T_{W}
$$

That condition occurs when all of the energy transfer to the liquid film goes into the vaporization of the liquid in the film*. Moreover, at the point downstream from the point of liquid injection where that condition is obtained, the temperature $T_{l}\left(=T_{W}=T_{S}\right)$ is a maximum. That maximum liquid temperature is denoted herein by $T_{1, m}$.

The maximum liquid temperature, $T_{1, m}$, was determined experimentally for the present investigation by measuring $T_{1,2}$, the temperature for the liquid coolant at the point of liquid withdrawal. Recall that the attempt was made in the subject investigation to preheat the liquid before injection so that the sensible enthalpy rise that would occur for the liquid after it was introduced onto the test plate wculd

*That is, none of the energy transfer to the surface of the liquid film goes to increase the sensible enthalpy for the liquid in the film. 
be minimized. Therefore, for those tests where it was possible to completely preheat the liquid before injection, it follows that the temperatures $T_{1, m}$ and $T_{1,2}$ were equivalent. Moreover, for those experimental tests wherein the liquid could not be completely preheated before injection (due to limitations in the heating capacity of the heat exchanger that was employed), it was found that the measured withdrawal temperature was not influenced significantly by the rate at which liquid was injected onto the test plate, thereby suggesting that the rate of energy transfer to the liquid film was sufficiently rapid to cause the allowable increase in the sensible enthalpy for the liquid to occur before the liquid film reached the capture slot. It follows, therefore, that the temperaturess $T_{1,2}$ and $T_{1, m}$ could be reasonably equated for all of the experimental tests of the subject investigation.

The liquid temperature $T_{1,2}$ was measured with a chromelalume 1 thermocouple*, and the indicated temperature was continuously recorded on a stripchart recorder. Experimental values for $T_{1,2}$ were obtained for each of the four liquids that was employed, and for the entire range of values for the gas stream parameters that was investigated**. Table G.l of Appendix $G$ lists the average value of $T_{1,2}$ for each of the experimental tests that are reported herein. Also,

* See Fig. B.6 of Appendix B for the physical location of the thermocouple:

** Refer to Tabl: 1 in Section 2.3. 
there is a graphical way in which the data can be presented that is of interest. In most previous investigations of liquid-film cooling (e.g., 20, 22, 23), the maximum temperature for the liquid film was taken to be the boiling temperature for the liquid at the prevailing pressure. However, it is demonstrated theoreticaliy in Refs. (21) and (26), and in section 3.4 of this report, that the temperature of the liquid film can never be equal to that boiling temperature. Figure 18 graphically illustrates this point for the data obtained in the subject investigation. Presented in Fig. 18 is a plot of the boiling temperature for the liquid at the prevailing pressure versus $T_{1,2}$, the measured liquid-film temperature. Data are presented for water, methanol, butanol, and RP-1. Figure 18 shows that the maximum film temperature can be substantially less than the boiling temperature at the prevailing pressure. The maximum difference between these two temperatures encountered in the present investigation is approximately $355 \mathrm{~F}$, and it was realized for RP-1 at a pressure of $150 \mathrm{psia}$ and a gas stream temperature of $400 \mathrm{~F}$. 


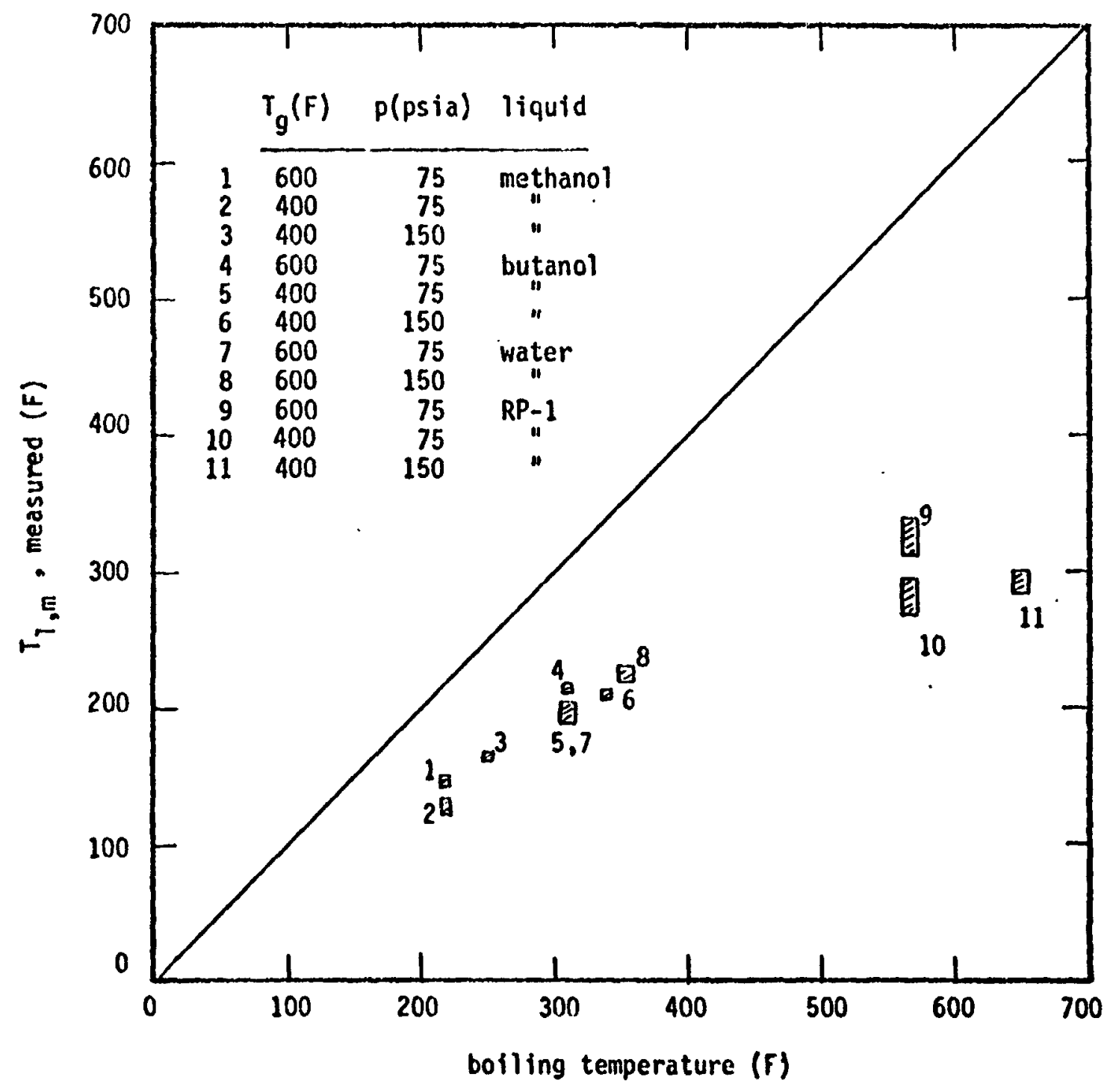

Fig. 18. Comparison of Maximum Liquid Temperature and Boiling Temperature 


\section{CORRELATION OF THE EXPERIMENTAL DATA}

The present section is concerned with the correlation of the experimental data that were discussed previously in section 2. It should be stated at the outset that it was the intention of the present investigation to develop formulae that could be employed to accurately design a liquidfilm cooled system but which would not involve excessive computations. Therefore, the present investigation sought to consider the details of the complex phenomena that characterize the interaction of a high velocity gas stream and a thin liquid film only to the exteni necessary to develop those design formulae. These interfacial phenomena have been the object of a large number of experimental and/or analytical investigations reported in the literature. To illustrate, references $(5,32)$ were concerned with the amplitude and wave length spectra that the gas-liquid interface exhibits for various liquid and gas stream flow conditions; references $(33,34)$ were concerned, in part, with applying stability theory to analyze the phenorenon of fiim instability; and references $(28,35)$ investigated the structure of the boundary layer which develops over the surface of a liquid film. It is virtually impossible; however, to 
utilize these very interesting data--particularly in a quantitative serise--to answer the basic engineering question: what is the net rate of mass transfer from a thin liquid film to a high velocity, hot gas stream? The relatively simple formulae developed herein answer that question explicitly.

Section 3.1 presents a correlation of the data for mass transfer with a constant velocity gas stream. Correlation of the data for mass transfer where the gas stream was accelerating over the wetted test surface is discussed in Section 3.3 Analys is of the latter data requires first the consideration of the problem of how the net rate of mass transfer varies with the liquid-film cooleu lenght 1; Section 3.2. considers that problem. Section 3.4 presents an analys is of the data for the maximum liquid temperature.

\subsection{Correlation of the Data for Mass Transfer for a Constant Velocity Gas Strean}

3.1.1. General Discussion

The experimental data for mass transfer for the subject investigation showed that for fixed gas stream flow conditions, the rate of liquid withdrawal, $m_{2}$, sould be approximately expressed as a linear function of the rate of liquid 
injection, $m_{1}{ }^{*}$. Thus

$$
m_{2}=-A+B m_{1}
$$

If $m_{1}$ is subtracted from both sides of $E q .(3-1)$, the resultant expression can be written in the form

$$
m_{1}-m_{2}=A+(1-B) m_{1}
$$

Noting that the quantity $\left(m_{1}-m_{2}\right)$ is simply $m^{\prime}$, the net rate of mass transfer from the liquid film to the gas stream, we obtain from Eq. (3-2) the expression

$$
m^{\prime}=A+(1-B) m_{1}
$$

Equation (3-3) expresses the net rate of mass transfer from the liquid film as a linear function of the rate of liquid injection.

In the original statement of the problem in Section 1.2 , it was indicated that $m$ ' was made up of two fundamental contributions: (a) the contribution due to the evaporation of the liquid in the liquid film, and (b) the contribution due to the entrainment of bulk liquid into the gas stream. The problem, therefore, is to determine how each of these two contributions can be evaluated from Eq. (3-3). With

* Refer to Figs. 14, 15, and 16 and to Appendix 6 . 
reference to that problem, consider the following two points: (a) The phenomen of liquid entrainment generally has been associated with the shearing off of liquid droplets from large scale disturbance vaves (instability waves) on the surface of the liquid film. Therefore, it is reasonable to assume that the rate at which liquid is entrained fro the surface of these large scale disturbance waves into the gas stream would increase with the frequency of occurrence of these waves. It has been demonstrated (10) that the frequency of occurrence of these waves increases with $m_{1}$, the rate at which liquid is introduced onto the wetted wall surface. Therefore, the rate of entrainment of liquid into the gas stream should be proportional to $m_{1}$. (b) It can be argued that the evaporation contribution to the total rate of mass transfer from the iiquid film to the gas stream should be largely independent of the flow parameters for the liquid phase. The extent to which the turbulent transport of heat to the surface of the liquid film is intensified because of the effective roughness of the surface of the liquid film should be primarily a function of the physical scale of the substructure on the surface of the liquid film. The photographic data discussed in Section 2.4 suggested, however, that while the scale of that substructure is influenced to a considerable degree by the flow parameters for the gas stream (e.g., $u_{g}, G$, and $\left.M_{0}\right)$, it is not noticeably altered aither by sicnificant variations in the rate 
at which liquid is injected onto the test plate or by significant variations in the physical properties for the liquid phase. It thus seems reasonable to assume, at least as a first appioximation, that the evaporative contribution is not dependent on the parameters for the liquid phase.

The foregoing two points and the form of the Eq. (3-3) suggest the simple hypothesis that (a) the first term on the right-hand side of Eq. (3-3) represents the contribution to the total mass transfer rate that is due to the evaporation of the liquid in the film, and (b) the second term on the right-hand side of Eq. (3-3) represents the contribution that is due to the entrainment of bulk liquid from the surface of the liquid film into the gas stream. Thus, it is hypothesized herein that

A $=$ the evaporation contribution

(1-B) $m_{1}$ = the entrainment contribution

The ultimate justification for this simple hypothesis ic the fact that it resulted in a satisfactory correlation of the experimental data. This will be demonstrated in Subsection 3.1 .5 of the present report.

Referring again to the statement of the subject problem presented in Section 1.2, it was indicated that the evaporative contribution to the total rate of mass transfer 'm' could be subdivided into (a) that due to the simple mass transfer and (b) that due to the interfacial phenomena (the effective surface roughness). If the term $A$ in.Eq. (3-3) 
gives the total contribution to $\mathrm{m}^{\prime}$ that is due to evaporation, and if $m_{s}^{\prime}$ denotes the rate of simple mass transfer, then a parameter $r$, termed the roughness parameter, can be defined such that

$$
A=(1+r) m_{s}^{\prime}(1 b / \sec -f t)
$$

The parameter $r$ is a measure of the degree to which the interfacial structure intensifies the turbulent transport of heat from the gas strean to the surface of the liquid film. For the limiting case where the slirface of the liquid film becomes hydrodynamically smooth, the parameter $r$ goes to zero, and the problem reduces to the classical problem of simple mass transfer.

In addition to the roughness parameter $r$, it is convenient to define ar entrainment parameter es such that

$$
e_{0}=1-B
$$

where $B$ is defined by Eq. $(3-1)$. The discussion of section 2.5 suggests that $e_{0}$ is primarily a function of the surface tension $\sigma$ for the liquid phase and of the momentum parameter $M_{n}$ for the gas strean. The subscript "o" on $e_{n}$ indicates that $e_{0}$ was determined from experimental data for which the liquid-film cooled length was maintained constant at 10 inches $\left(1=1_{0}=10\right.$ inches $)$. Section 3.2 of the subject 
report shows how the experimental results obtained for that fixed film cooled length can be utilized to analyze the general case of liquid-film cooling where the iquid-film cooled length 1 is arbitrary.

Substitution of Eqs. (3-4) and 3-5) into Eq. (3-3) results in the following expression. Thus

$$
m^{\prime}=(1+r) m_{s}^{\prime}+e_{0} m_{1}
$$

Equation (3-6) is the primary equation that was utilized in the present investigation to correlate the net rate of mass transfer from the liquid film to the gas stream. The significance of $\varepsilon$ ach of the terms in Eq. (3-6) is shown graphically in Fig. 19. The lower solid curve in the figure represents a least-squares fit of the data for mass transfer that were realized for a typical experimental test. The upper solid curve in Fig. 19 represents the condition of no mass transfer, and the upper dashed curve is the curve that is predicted by the simple theory for mass transfer. The lower dashed curve represents the curve that would be predicted by the present theory, accounting for the roughness of the film, if there was no entrainment of liquid into the gas stream. Figure 19 shows how each of the terms in Eq. $(3-6), m_{s}^{\prime}, r m_{s}^{\prime}$, and $e_{0} m_{1}$, can be conveniently interpreted graphically.

Figure 19 and Eq. $(3-6)$ show that the subject problem 


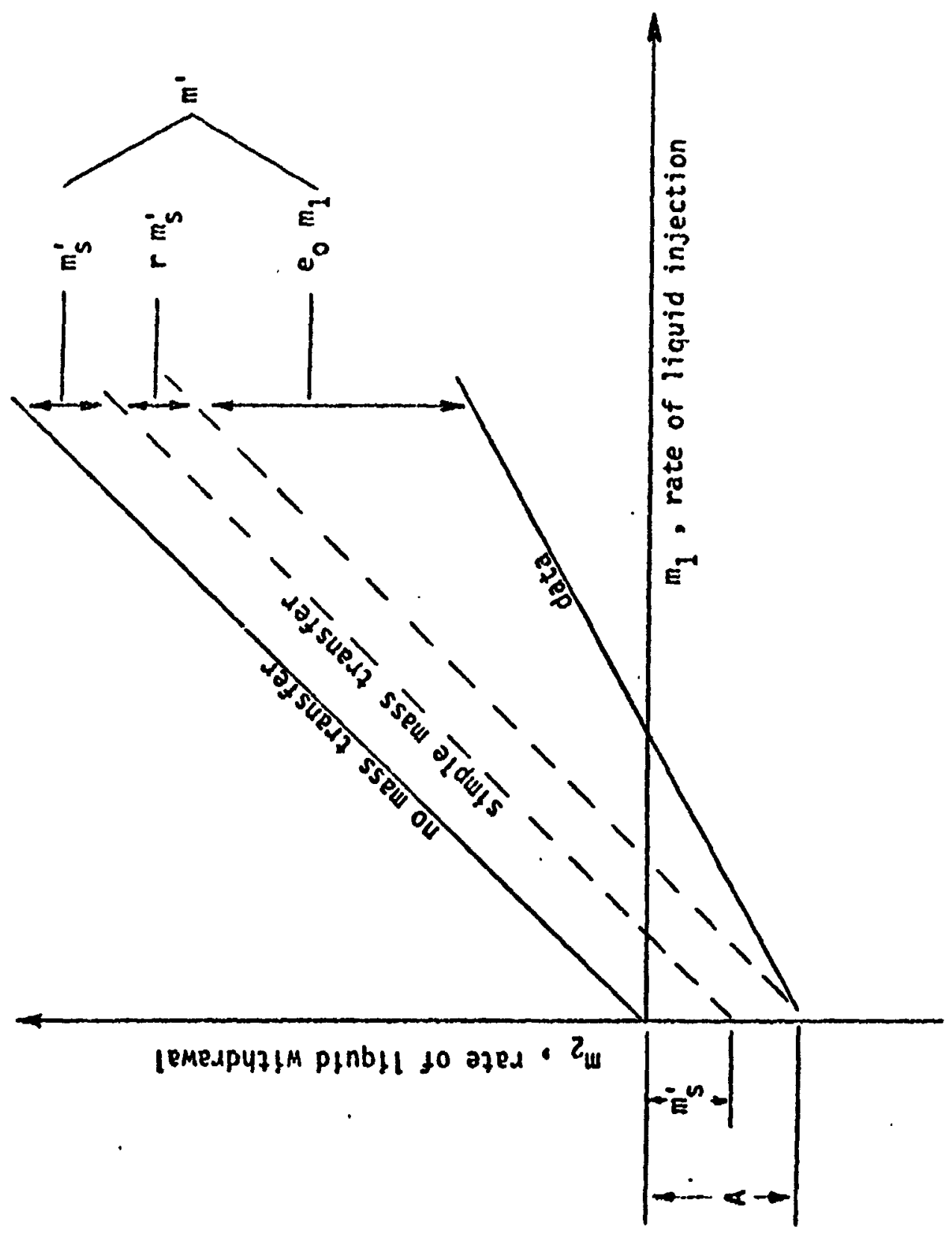

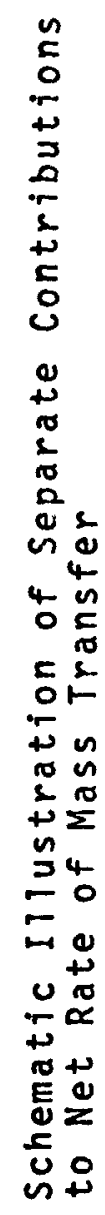


of correlating the experimental data for $m^{\prime}$ is reduced to that of calculating the simple mass transfer rate $m_{s}^{\prime}$ and to the correlation of the parameters $r$ and $e_{0}$ on the basis of the experimental data. Subsection 3.1 .2 discusses the method that was employed to calculate $m_{s}^{\prime}$ in the present investigation. Subsection 3.1 .4 and 3.1 .5 discuss the techniques for correlating the parameters $e_{0}$ and $r$, respectively, in terms of pertinent flow parameters. Subsection 3.1 .6 then illustrates the agreement of the resultant data correlation with the experimental mass transfer data of the present investigation.

\subsubsection{The Evaluation of the Rate of Simple Mass Transfer $m_{s}^{\prime *}$}

The net rate of simple mass transfer from the liquid film to the gas stream is related to the local rate of mass transfer by the following expression. Thus

$$
\begin{aligned}
m_{s}^{\prime} & =\int_{x_{1}}^{x_{2}} m_{s}^{\prime \prime} d x \\
& =\left(x_{2}-x_{1}\right) \bar{m}_{s}^{\prime \prime}=l_{0} \bar{m}_{s}^{\prime \prime}
\end{aligned}
$$

*The primary reference for this section is the textbook by Kays (36) on convective heat and mass transfer. 
where

$m_{s}^{\prime}=$ the net rate of simple mass transfer

$m_{s}^{\prime \prime}=$ the local rate of simple mass transfer

$1_{0}=$ the film cooled length ( = 10 iriches $)$

$\bar{m}_{s}^{\prime \prime}=\begin{aligned} & \text { the average value for } m_{s}^{\prime \prime} \text { over the film cooled } \\ & \text { length }\end{aligned}$

A method is outlined in the present section for the evaluation of the local rate of simple mass transfer $m_{s}^{\prime \prime}$. The method consists of two basic steps: (a) writing an energy balance across the gas-liquid interface so that $m_{s}^{\prime \prime}$ can be directly related to $\mathrm{q}_{S}$, the local rate of heat transfer from the hot gas stream to the surface of the liquid film, and (b) determining $q_{S}$ from the comprehensive theory that is presented by Kays (36) for that problem. The solution for $m_{s}^{\prime}$ is then completed by developing a closedform expression for the average rate of simple mass transfer, $\bar{m}_{s}^{\prime \prime}$, that appears in Eq. $(3-8)$.

The Energy Balance at the Gas-Liquid Interface. Figure 20 illustrates a general interfacial element placed at the surface of a stable, non-reactive liquid film with the dotted lines representing the surfaces considered in writing an energy balance for the element. The different energy fluxes involved are defined as follows:

(i) $q_{S}=(i \quad \partial T / \partial y)_{S}=$ the rate at wich energy is transferred across the S-surface by conduction.

(2) $q_{L}=(k \partial T / \partial y)_{L}=$ the rate at which energy is trans ferred across the L-surface by conduction. 


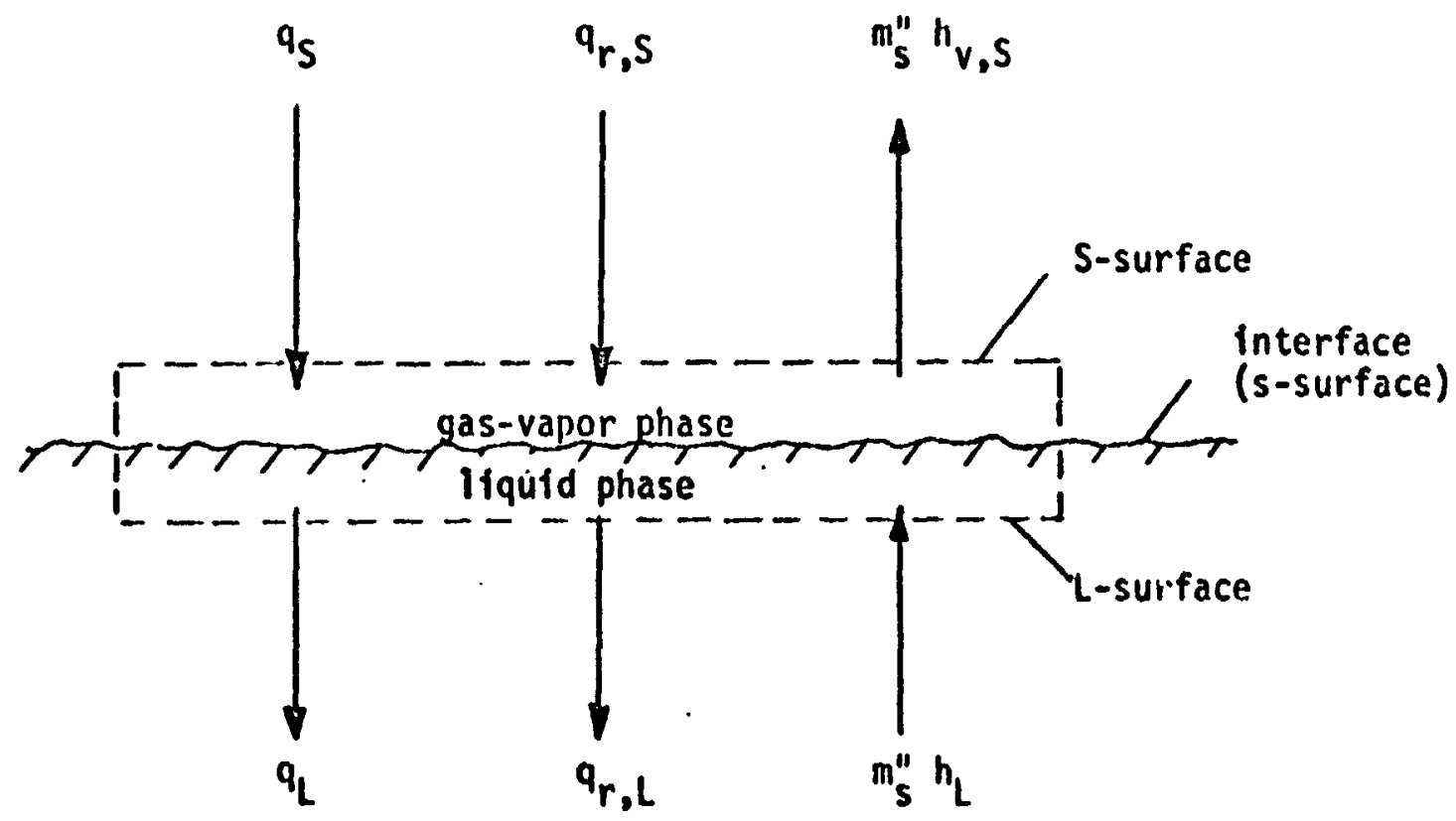

Fig. 20. Control Volume for Interfacial Energy Balance 


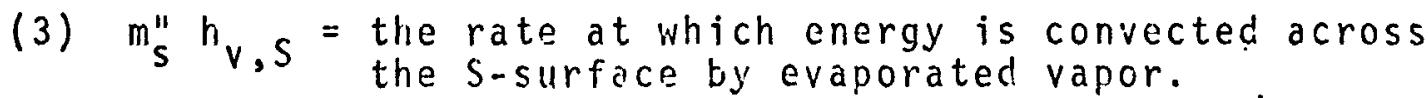

(4) $m_{s}^{\prime \prime} h_{L}=$ the rate at which energy is convected across the L-surface by the evaporating liquid. (5) ${ }^{q_{r, s}}=$ the rate at which energy is transferred across
the $s$-surface by radiation. (6) $a_{r, L}=$ the rate at which energy is transferred across
the L-surface by radiation.

The energy balance for the interface element illustrated in Fig. 20 can be written as follows:

$$
q_{I}^{q_{S}}=q_{L I}+m_{S}^{\prime \prime}\left(h_{V, S}-h_{L}\right)+\left(q_{r, L}-q_{\text {IV }} r_{S}\right)
$$

Term III in Eq. (3-9) can be rewritten in the form

$$
m_{s}^{\prime \prime} H_{v}
$$

where $H_{V}$ is the latent heat of vaporization for the liquid coolant which corresponds to the saturation condition that is prescribed by the film surface temperature $T_{s}$. Furthermore, for the temperature levels encountered in the subject investigation, term IV in Eq. (3-9) can be considered negligible in comparison to a term such as term III. Thus, neglecting that term, Eq. (3-9) can be rewritten to yield

$$
q_{S}=q_{L}+m_{S}^{\prime \prime} H_{V}
$$

The term $q_{L}$ in Eq. $(3-10)$ is that portion of the heat transfer to the surface of the liquid film which crosses the 
interface and goes into raising the sensible enthalpy of the liquid and/or is transferred through the wetted plate to the surroundings. In the subject investigation, the test plate was essentially adiabatic so that the heat transfer across the test plate to the surroundings was negligible. Moreover, an attempt was made in the investigation to preheat the liquid before injection so that no rise in sensible enthalpy would occur for the liquid once it was introduced onto the test plate. The elimination of the rise in sensible enthalpy for the liquid would make the quantity $q_{L}$ in Eq. (3-10) equal to zero and thus simplify the energy balance as mentioned in Section 2.2. However, due to limitations in the heating capacity of the heat exchanger employed to preheat the liquid, some rise in the sensitle enthalpy for the liquid usually occurred after the liquid was injected. To correct for that fact, the term $q_{L}$ in Eq. (3-10) was approximated by the expression*

$$
q_{L}=m_{5}^{\prime \prime}\left(h_{1,2}-h_{1,1}\right)
$$

where

$$
\begin{aligned}
h_{1,2}= & \text { sensible enthalpy for the liquid at the point } \\
& \text { of withdrawal, } x=x_{2} \\
h_{1,1}= & \text { sensiole enthalpy for the liquio at the point } \\
& \text { of injection, } x=x_{1}
\end{aligned}
$$

*This approximation is the same as that employed in Refs. $(19,29)$. 
Substitution of Eq. $(3-11)$ into Eq. $(3-10)$ results in the following basic expression for the interfacial energy balance. Thus

$$
\frac{q_{S}}{m_{s}^{\prime \prime}}=H_{v}+h_{1,2}-h_{1,1}
$$

It is convenient to introduce a parameter $\phi$ that is termed herein the energy balance parameter, where $\phi$ is defined as the group of terms on the right-hand side of Eq. (3-12). Thus

$$
\phi=H_{v}+h_{1,2}-h_{1,1}
$$

or $\phi=\frac{q_{s}}{m_{s}^{\pi}}$

Since the energy balance parameter $\phi$ is expressed solely in terms of quantities that can readily be evaluated, the problem of determining $m_{s}^{\prime \prime}$ thus reduces to that of evaluating $\mathbf{q}_{\mathrm{S}}$

The Evaluation of ${ }^{q_{S}}$. It is convenient to express $q_{S}$ in terms of $\mathrm{St}_{s}$, the local stanton number for simple heat transfer, where $s t_{s}$ is defined by

$$
S t_{s}=\frac{q_{S}}{G C_{p g}\left(T_{g}^{-T_{s}}\right)}
$$


where

$G=$ the mass velocity for the gas stream

$c_{F g}=\begin{aligned} & \text { the specific heat at constant pressure for the } \\ & \text { gas stream }\end{aligned}$

$T_{g}=$ the temperature for the gas stream

$T_{S}=$ the temperature at the surface of the liquid film

The complete evaluation of the local stanton number sts requires that the effect of the folluiving on the transport phenomena be accounted for: (a) the thermal entrance length $x_{t}=x_{1}$, (see Fig. 6); (b) the dependence of the physical properties for the gas phase on the temperature $T$ and on the local concentration of the injected vapor $c ;(c)$ the rate of transfer of vapor from the surface of the liquid film into the gas phase; and (d) non-unity values for the Prandtl number $\mathrm{Pr}$ for the gas phase. If $\mathrm{St}_{\text {so }}$ denotes the local value for the stanton number for the case where none of these effects are present, then the stanton number $s t$ can be related to $\mathrm{St}_{\text {so }}$ through a number of correction factors that are denoted by $F$. Thus

$$
s t_{s}=F_{x_{t}} F_{T} F_{C} F_{m} F_{P r} S t_{s o}
$$

where

$$
\begin{aligned}
& F_{X_{t}}= \text { the correction factor for themm? antrance } \\
& \text { length effects }\left(x_{t}=x_{1}\right) \\
& F_{T}= \text { the correction factor for the dependence of the } \\
& \text { physical properties of the gas on the temperature }
\end{aligned}
$$




$$
\begin{aligned}
F_{C}= & \text { the correction factor for the dependence of the } \\
& \text { physical properties for the gas on the concen- } \\
& \text { tration of the injected vapor } c \\
F_{m "}= & \text { the correction factor for the injection of the } \\
& \text { mass (i.e., vapor) into the developing boundary } \\
& \text { layer* } \\
F_{P r}= & \text { the correction factor for a non-unity Prandt1 } \\
& \text { number for the ods stream }
\end{aligned}
$$

Kays (36) suggests that for the case where the gas stream velocity is constant**, the correction factors in Eq. (3-16) can be evaluated by the following empirical equations. Thus

$$
\begin{aligned}
& F_{x_{t}}=\left(1-\frac{x_{t}}{x}\right) \\
& F_{T}=\left(\frac{T}{T}\right) \\
& \left.\frac{T}{g}\right)^{-0.25} \\
& F_{C}=\left(\frac{M_{s}}{M_{g}}\right) \\
& F_{m^{\prime \prime}}=\frac{\ln \left(1+B_{h}\right)}{B_{h}} \\
& F_{P r}=(\operatorname{Pr})^{-0.4}
\end{aligned}
$$

where

$M_{g}=$ the molecular weight for the gas stream.

$M_{s}{ }^{\prime}=$ the molecular weight for the. gas-vapor mixture at the surface of the liquid film

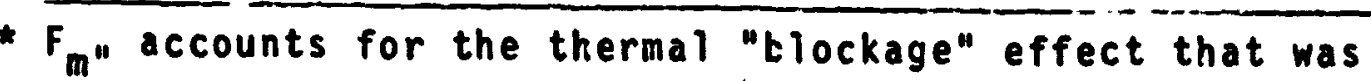
discussed in Section 1.3 .

**This restriction actually applies to only $F_{x_{t}}$. 
$B_{h}=a$ dimensionless heat-mass transfer pärameter

$$
\equiv \frac{c_{p g}\left(T_{g}-T_{s}\right)}{\phi}
$$

To complete the solution to $q_{S}$, both the term $s t_{s o}$ in Eq. $(3-16)$ and the term $M_{S}$ in Eq. (3-19) must be evaluated. The following classical expression for turbulent heat transfer to a flat plate was employed to evaluate St $_{\text {so }}(36)$. Thus

$$
S t_{\text {so }}=0.0295 \operatorname{Re}_{x}^{-0.2}
$$

where

$\begin{aligned} \operatorname{Re}_{x}= & \text { the Reynolds number for the gas stream based } \\ & \text { on the characteristic length } x\end{aligned}$

$$
\equiv \frac{G x}{\mu_{g}}
$$

$\mu_{g}=$ the dynamic viscosity for the gas stream evalu-

The molecular weight $M_{s}$ is related to $M_{v}$, the molecular weight for the vapor, and $M_{g}$, the molecular weight for the free-stream gas, by

$$
\frac{1}{M_{s}}=\frac{C_{s}}{M_{v}}+\frac{\left(1-C_{s}\right)}{M_{g}}
$$

* If compressibility effects are important (Mach number greater than, say, 0.5), then the temperature difference $\left(T_{g}-T_{s}\right)$ should be replaced by $\left(T_{g}\right.$,aw $\left.-T_{s}\right)$, where $T_{g, a w}$ is the adiabatic-wall recovery temperature for the gas itream. 
where

$$
\begin{aligned}
& c_{s}=\text { the concentration for the vapor at the surface } \\
& \text { of the liquid film }
\end{aligned}
$$

The vapor concentration $C_{s}$ can be determined by the following approximate expression (36). Thus

$$
c_{s}=\frac{{ }^{B} h}{1+B_{h}}
$$

The Evaluation of $m_{s}^{\prime}$. Equations $(3-14),(3-15),(3-16)$ and (3-22) can be combined to obtain the following basic expression for $m_{s}^{\prime \prime}$ as a function of the coordinate $x$. Thus

$$
m_{s}^{\prime \prime}=G B_{h} F_{x_{t}} F_{T} F_{C} F_{m} " F_{P r} S t_{s o}
$$

For the subject case where the gas stream velocity is constant over the film cooled length $1_{0}$, it can be reasonably assumed that only the terms $F_{x_{t}}$ and $S t_{\text {so }}$ in Eq. $(3-27)$ are a function of the coordinate $x$. Therefore, for that special case, the result of the substitution of Eq. (3-27) into Eq. $(3-7)$ can be written as

$$
m_{s}^{\prime}=G B_{h} F_{T} F_{C} F_{m} " F_{P r} \int_{x_{1}}^{x_{2}} F_{x_{t}} s t_{s o} d x
$$

I 6 is not pussible to evaluate the integral in tq. $(3-28)$ in 
closed form if Eq. $(3-17)$ is substituted for $F_{x_{t}}$. However, Reynolds, Kays, and Kline (37) have developed the following approximate expression for that integral term:

$$
\int_{x_{1}}^{x_{2}} F_{x_{t}} s t_{s 0} d x=\left\{\frac{5}{4} \frac{\left(\frac{x_{2}}{x_{1}}\right)^{0.2}\left[\left(\frac{x_{2}}{x_{1}}\right)^{0.9}-1\right]^{\frac{8}{9}}}{\left[\left(\frac{x_{2}}{x_{1}}\right)-1\right]}\right\}_{0} s t_{s 0,2}
$$

where $S t_{s o, 2}$ is the value for $S t_{\text {so }}$ that is obtained from Eq. (3-23) with $x=x_{2}$. For a value of $x_{1}=40$ inches and a value of $x_{2}=50$ inches, the non-dimensional term inside the brackets \{\} in Eq. (3-29) has a value of 1.374. Substituting that value into Eq. $(3-29)$ and the resultant expression into Eq. $(3-28)$, one obtains the basic expression that was employed in the present investigation to evaluate the rate of simple mass transfer from the liquid film into the constant velocity gas stream. Thus

$$
m_{s}^{\prime}=1.374 G B_{h} F_{T} F_{C} F_{m} " F_{P r} 1_{0} S t_{s 0,2}
$$

Table G.l of Appendix $G$ lists the average value for the simple rate of mass transfer, $m_{s}^{\prime}$, that was calculated for each experimental test. The range of values inat was computed for each of the dimensionless terms in Eq. (3-30) and also for the interfacial concentration $C_{s}$ and the heat-mass. 
transfer parameter $B_{h}$ is summarized as follows:

$$
\begin{array}{ll}
F_{T} & =1.015 \text { to } 1.150 \\
F_{C} & =0.976 \text { to } 1.096 \\
F_{m "} & =0.86 \text { to } 0.96 \\
F_{P r} & =1.168 \text { (for a11 cases) } \\
S_{\text {so,2 }} & =0.0021 \text { to } 0.0030 \\
{ }_{\text {'s }} & =0.081 \text { to } 0.247 \\
B_{h} & =0.083 \text { to } 0.328
\end{array}
$$

3.1.3 The Correlation of the Entrainment Parameter $e_{0}$

The present section is concerned with the correlation of the entrainment parameter $e_{0}$ that occurs in the primary equation $(3-6)$. The procedure that was employed herein to develop the correlation was purely inductive. That is, the procedure that was employed was to first note how each of the pertinent flow parameters influenced the rate of entrainment of liquid from the liquid film into the gas stream, and to then develop a general correlation that would reflect each of those observations. Thus an analytical model is not postulated in this report for the approximate analysis of the entrainment phenomenon. Moreover, the method of dimensional analysis was not directly employed to develop the subject correlation for $e_{n}$. The ultimate justification for the simple procedure that was employed is the fact that it resulted in a satisfactory correlation of the parameter $e_{0}$ in terms of the pertinent variables for both 
the gas and liquid phases. The next subsection of this report, subsection 3.1.4, does, however, consider in some detail the possible physical significance of the resultant correlation that was developed for $e_{0}$. The discussion in Subsection 3.1 .4 suggests a simple procedure for correlating the roughness parameter " $r$ " that was defined by Eqs. (3-4) and $(3-6)$. Subsection $3.1: 5$ presents the correlation for the parameter $r$.

The discussion of the experimental data for mass transfer that was presented in section 2.5, and summarized in Subsection 2.5.3, showed that the two parameter's which most influenced the net rate of entrainment of liquid from the liquid film into the gas stream were the momentum parameter $M_{0}$ for the gas stream and the surface tension o for the liquid phase. The rate of entrainment of liquid increased with increasing values for $M_{0}$ and with decreasing values for $\sigma$. In view of those observations, the attempt was made to correlate the parameter $e_{0}$ in terms of a dimensional entrainment group " $X_{e}$ " which is defined as*

$$
x_{e}=\frac{M_{0}{ }^{a}}{\sigma}\left(\frac{T^{T}}{T_{s}}\right)^{\frac{a}{2}}
$$

where "a" is a constant that was determined experimentally. The term $\left(T_{g} / T_{s}\right)^{a / L}$ in Eq. $(3-31)$ arises from the substitution for the gas stream density $\rho_{g}$ in the parameter $M_{0}$ of the

*Evidence is presented in Subsection 3.1.4 that $x_{e}$ is equivalent to a dimensioniess Weber number. 
corrected density $\rho_{g}\left(T_{g} / T_{s}\right)^{\frac{3}{2}}$. This is a technique that is frequently employed in the correlation of heat transfer data to account for the fact that the density for the gas within the boundary layer region is inversely proportional to the temperature for the gas (36)*. It should be noted, however, that because of the low gas stream temperatures that were employed in the subject investigation, the term $\left(T_{g} / T_{s}\right)^{a / 2}$ was not crucial to the correlation of the experimental data for the entrainment parameter $e_{0}$ (discussed immediately below). It is included in the definition of the entrainment group $x_{e}$ primarily to be consistent with the accepted practice for accounting for variable property effects.

A lengthy trial-and-error procedure employing all of the experimental data presented herein showed that the form of the entrainment group presented in Eq. (3-31) resulted in the best correlation of the data over the complete range of parameters investigated, and that the parameter $e_{0}$ could be -orreiated satisfactorily if the constant "a" in Eq. (3-31) was set equal to $\frac{1}{2}$. Figure 21 presents the resultant correlation of the data for $a=\frac{1}{2}$. Presented in the figure is $\left(1-e_{0}\right)$ as a function of $x_{e}$, where $\left(1-e_{0}\right)$ was determined for each experimental test from the slope of the least-squares fit curve for the experimental data of that test (e.g., as shown in Fig. 14). The nominal values for the gas stream temperature and pressure are indicated in Fig. 21, together

*This technique was employed, in part, to derive the expression for the aforementioned correction factor $F_{T}$ (see Eq. $(3-18))$. 


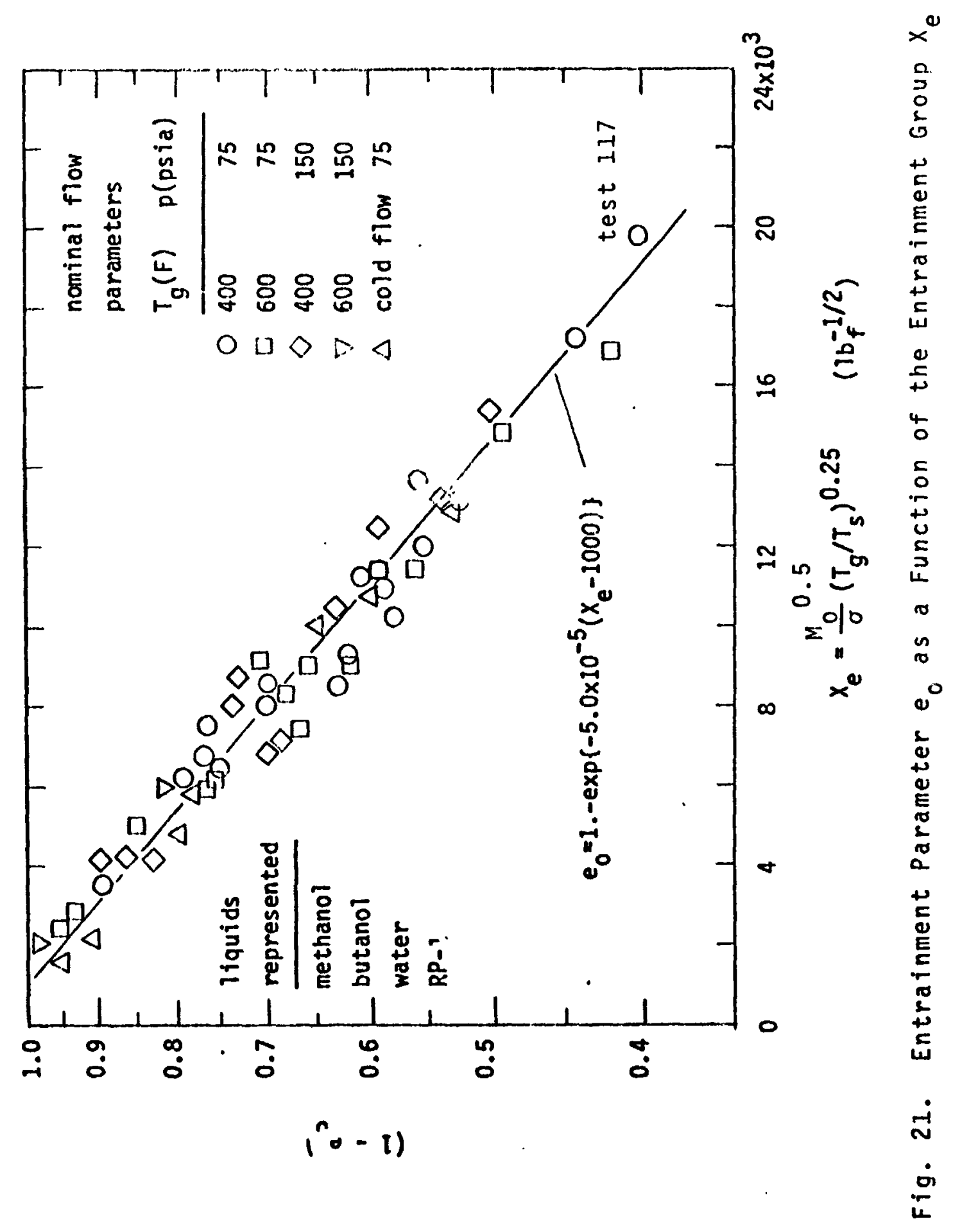


with the four liquids that are represented by the data. Although there is admittedly some scatter in the experimental results, some of the scatter can be attributed to the fact that $\left(1-e_{0}\right)$ was determined from a least-squares fit of the "raw" experimental data for mass transfer. Indeed, because the data presented in Fig. 21 do represent such a substantial variation in both the pertinent parameters for the 1iquid phase and for the gas phase, it is somewhat surprising that $e_{0}$ can be correlated so well in terms of the relatively simple parameter $X_{e}$. Perhaps most significant is the fact that $e_{0}$ was not found to show any dependence on the liquid viscosity $\mu_{1}$. The data presented in $F_{i g} .21$ represent a variation in $\mu_{1}$ from $1.7 \times 10^{-4} \mathrm{lb} / \mathrm{ft}-\mathrm{sec}$ for water at a temperature of $265 \mathrm{~F}$ to $30 \times 10^{-4} \mathrm{lb} / \mathrm{ft}-\mathrm{sec}$ for butanol at a temperature of $40 \mathrm{~F}$. The results of the subject investigation are thus contrary to the suggestion made by some investigators that a liquid-film Reynolds number (which is inversely proportional to $\mu_{l}$ ) is the parameter that most characterizes the entrainment phenomenon*.

The curve shown in Fig. 21 is given by the following expression. Thus

$$
e_{0}=1.0-\exp \left[-5 \times 10^{-5}\left(x_{e}-1000\right)\right]
$$

where $x_{e}$ is substituted with the dimensions $1 b_{f}^{-\frac{1}{2}}$. Equation * Refar to the previous discussion on this point in subsection 2.4.1. 
(3-32) suggests that a critical value for $x_{e}$ of $10001 b_{f}^{-\frac{1}{2}}$ exists, below which no entrainment of liquid occurs. The critical or limiting value for $x_{e}$ of $10001 b_{f}^{-\frac{1}{2}}$ was determined partly from the experimental data of the present investigation, but also from the experimental data due to Steen and Wallis $(37,38)$. They considered in some detail the problem of determining the critical flow conditions at which entrainment from a liquid film first occurs. As a result of their experiments, it was suggested that a critical gas stream veiocity could be defined by the condition that

$$
\pi_{c}=2.5 \times 10^{-4}
$$

where

$$
\begin{aligned}
& \pi=\frac{u_{g} \mu_{g}}{\sigma}\left(\frac{\rho_{g}}{\rho_{1}}\right) \\
& 0.5 \\
& \rho_{1}=\text { liquid density } \\
& c \quad \text { denotes critical value }
\end{aligned}
$$

Recognizing that

$$
\rho_{g}^{0.5} u_{g}=M_{0}^{0.5}
$$

it follows that the parameters $x_{e}$ and $\pi$ are related by the euqation

$$
x_{e}=\frac{0.0 .5}{\mu_{g}} \pi
$$


The data reported by Steen et al. were for cold air flow, with most of the data obtained for water as the liquid phase. Assuming that the temperature of both the gas stream and the liquid film was approximately $60^{\circ} \mathrm{F}$, and substituting the corresponding values for $\mu_{g}$ for air and $\rho_{1}$ for water at that temperature, Eq. $(3-26)$ gives the following relationship between $x_{e}$ and $\pi$ for the data of steen et al. Thus

$$
x_{e}=3.8 \times 10^{6} \pi\left(1 b_{f}^{-\frac{1}{2}}\right)
$$

Substitution of the critical value for $\pi$ that was proposed by Steen and Wallis into Eq. (3-37) results in the corresponding critical value for $x_{e}$ of $9501 b_{f}^{-\frac{1}{2}}$. The critical value of $1000 \mathrm{Ib}_{f}^{-\frac{1}{2}}$ for $x_{e}$ in Eq. (3-32) was selected as a convenient compromise between the data of the present investigation and those for the investigation of steen et al.

The experimenial data of Steen and Wallis $(37,38)$ for the onset of entrainment, though obtained under much less severe flow conditions than those that characterized the present investigation, are in good agreement with the data of the subject investigation. It is important to note, however, that it was found that it was not possible to correlate the experimental data of the present investigation for $e_{0}$ in terms of $\pi$ instead of in terms of $x_{e}$. Unlike the investigation due to steen et 21 , the gas stream viscosity $\mu_{g}$ for the present investigation varied substantially. 
Although the parameter $\pi$ does have the advantage over the parameter $x_{e}$ of being dimensionless, the successful correlation of the data of the subject investigaion suggests that the parameter $x_{e}$ better characterizes the phenomena. It will be argued in the following subsection that although the parameter $x_{e}$ is not itself a dimensionless variabie, the correlation of $e_{0}$ in terms of that parameter is equivalent to the correlation of $e_{0}$ in terms of a timensionless weber number.

It is interestir. " note from Fig. 21 that for the present investigation the entrainment parameter $e_{0}$ varied from essentially zero to a maximum value of approximately 0.6. The maximum value for $e_{0}$ that is shown in Fig. 21 corresponds to the experimental test 117 where methanol was employed as the film coolant. For that test, the computed simple rate of mass transfer was $0.022 \mathrm{lb} / \mathrm{sec}-\mathrm{ft}$ and the maximum mass transfer rate that was realized experimentally was $0.154 \mathrm{lb} / \mathrm{sec}-\mathrm{ft}$, a difference of 700 percent. Almost all of that difference (about 690 percent) was due to the entrainment phenomenon. In fact, it is important to note that with the exception of a very smail number of tests (most of which employed water as the film coolant which has a relatively high value of surface tension), the contribution to the total rate of mass transfer that was due to the entrainment of liquid into the gas stream was substantially larger than the contribution that was due to evaporation. 
This was true considering both the simple rate of avaporative mass transfer and the evaporative mass trunsfer that was due to the phenomeron of interfacial roughness.

\subsubsection{The Physical Significance \\ of the Correlation for $e_{0}$}

The physical significance of the satisfactory correlation of $e_{0}$ in terms of the parameter $x_{e}$ is discussed in the present section. The discussion will suggest a procedurs that is employed in subsection 3.1 .4 to correldte the roughness parameter $r$.

Since it is generally accepted that the phenomenon of entrainment is primarily the result of the shearing off of liquid droplets from disturbance waves on the liquid film surface, it is instructive to consider the forces that act on a wave that develops on the surface of the liquid film. If $I_{s}$ characterizes the scale of those interfacial waves, then the aerodynamic drag force that is exerted by the gas stream on the wave that acts to destroy the wave is proportiona? to $M_{0}{ }^{2}$, and the force that is due to the surface tension that acts to keep the wave intact is proportional to $\mathrm{Ol}_{\mathrm{s}}$. The balance between these two forces should be the controlling factor in the breakdown of a disturbance wave on the surface of the liquid film. Therefore, that balance of forces should he important in determining the rate at which liquid is entrained from the liquid film into the gas scream. Thus, if a dimensionless Weber number, denoted by He, is 
defined as the ratio of those two forces such that

$$
\text { We }=\frac{M_{0}{ }^{\prime} s}{\sigma}
$$

it is reasonable to assume that the entrainment parameter $e_{0}$ could be correlated as

$$
e_{0}=e_{0}(W e)
$$

The results of the foregoing subsection showed that

$$
e_{0}=e_{0}\left(x_{e}\right)
$$

Upon comparing the defining equations for $x_{e}$ and $W_{e}$, and ignoring for the sake of argument the term $\left(T_{g} / T_{s}\right)^{\frac{1}{4} *}$, it is seen that the apparent contradiction between Eq. (3-39) and Eq. $(3-40)$ could be reconciled if a dimensional constant " $K$ " existed such that

$$
K=I_{S} M_{0}^{\frac{1}{2}}=\text { constant }
$$

There is, in fact, some physical evidence presented herein that supports the assumption that the dimensional constant $K$ does exist. Recall from the qualitative anaiysis of the photographic data in section 2.4 of this report that the *The maximum value of this term for the subject investigation was 1.15. 
parameter $M_{0}$ (and not $G$ or $u_{g}$ or any parameter for the liquid phase) was the parameter that most characterized the scale of the waves on the surface of the liquid film. Moreover, the photographs showed that the scale decreased with increasing values for $M_{0}$. Therefore, although the validity of the exponent of $\frac{1}{2}$ in Eq. (3-4I) cannot be argued on the basis of the qlalitative photographic data, there is some physical evidence to support the form of the equation.

Another interesting observation can be made with roference to Eq. $(3-41)$ that gives some insight into the phenomena. It is well documented $(18$, p671) that the shattering (or atomization) of liquid drops that are suspended in a gas stream can be characterized by a dimensionless Weber number, where the characteristic length ( $1_{s}$ in Eq. (3-38)) is chosen as the radius of the drop. If that weber number is greater than about 6 , the liquid drop will break down into smaller drops. How, if an analogy is drawn between the breakdown of such liquid drops and the breakdown of the liquid waves on the surface of the liquid film, then a crude calculation can be made for the constant $K$ in Eq. (3-41). Assuming that the critical value for We for the case where $I_{s}$ characterizes the scale of the waves on the surface of the liquid film is still 6, and recalling that the critical value for the parameter $x_{e}$ below which no entrainment of liquid occurred was approximately $1000 \mathrm{lb}_{f}^{-\frac{1}{2}}$, then $K$ can be 
approximated by the following calculation. Thus

$$
k=\frac{W_{e}}{X_{e}}=\frac{W_{e}}{X_{e, c}}=\frac{6}{1000}=0.0061 b_{f}^{\frac{3}{2}}
$$

Continuing this heuristic argument, it is interesting to use the above value for $K$, together with Eq. (3-41), to estimate the range of values for the scale $I_{s}$ for the subject investigation. From Table G.I of Appendix $G$, the maximum and the minimum experimental value for the momentum parameter $M_{0}$ was $10871 b_{f} / f^{2}$ and $201 b_{f} / f t^{2}$, respectively. Thus, employing the value for $k$ of $0.0061 b_{f}^{\frac{1}{2}}$, the maximum and the minimum value for the scale $I_{s}$ for the subject investigation can be estimated from Eq. $(3-41)$. Thus

$$
\begin{aligned}
& 1_{s, \max }=0.00135 \mathrm{ft} \\
& 1_{s, \min }=0.00018 \mathrm{ft}
\end{aligned}
$$

While these calculations are admittedly very approximate, the values obtained for the scale $1_{s}$ are surprisingly similar to the dimensions that characterize the structure of a liquid film that is subjected to relatively large interfacial shear forces (as is the case in the present investigation). For example, Ref. (19) shows that the mean thickness of such a liquid film is of the order of $10^{-4} \mathrm{ft}$. Moreover, the wave length of the small scale disturbances can be roughly approximated as 10 times the mean thickness of the liquid film $(8,5)$ or the order of $10^{-3} \mathrm{ft}$, and the amplitude of those disturbance waves is of the same order as the thickness of the 
liquid film (5), or $10^{-4} \mathrm{ft}$. The precedin: calculations, therefore, add scme additional support to the assumption that the dimensional constant $k$ exists. Moreover, they also suggest that the breakdown of the interfacial waves and the resultant entrainment of liquid into the gas stream may be somewhat analogous to the acomization of liquid drops that are suspended in a gas stream.

To summarize briefly, the above discussion suggests that (a) the dimensional parameter $x_{e}$ and the dimensioniess Weber number We are physically equivalent parameters, and (b) a constant $K=1_{s} M_{0}^{\frac{1}{2}}$ exists such that the characteristics (really, the gross characteristics) of the interfacial film structure can be characterized in terms of the flow parameters for the gas strean. The second of these two points, wich follows directiy from the first, is most pertinent to the correiation of the roughness parameter $r$ that is presented in the following section.

\subsubsection{The Correlation of the Roughness Parameter $r$}

The present section is concerned with the correlation of the roughriess parameter $r$ that was defined by Eqs. (3-4) and $(3-6)$. The primary assumption that was employed to develop the correlation was that, at least to a first approximation, the degree to which the effective roughness of the surface of the liquid film intensifies the turbulent transfer of energy from the hot gas stream to the surface 
of the liquid film is primarily a function of the scale of the small disturbance waves that develop on the surface of the film. That is, it was assumed that the roughness parameter $r$ could be correlated in the form

$$
r=r\left(l_{s}\right)
$$

Unfortunately, the utility of Eq. $(3-43)$ is limited because of the problems inherent in trying to measure the scale $1_{s}$. Indeed, there is considerable uncertainty as to what physical dimension for the waves on the surface of the liquid film corresponds to the length $1_{s}$. However, an argument was presented in the foregoing subsection that the scale $1_{s}$ could be approximately related to the momentum parameter $M_{0}$ by the following expression. Thus

$$
1_{s}=\frac{K}{M_{0}^{\frac{1}{2}}}
$$

Therefore, if a roughness group " $x_{r}$ " is defined by

$$
x_{r}=M_{0}^{\frac{3}{2}}\left(\frac{T_{g}}{T_{s}}\right)^{\frac{1}{4}}
$$

Equation (3-43) can be rewritten in the following equivalent form. Thus

*Again, as for Eq. (3-33) in Subsection 3.1.3, the term $\left(T_{g} / T_{s}\right)^{\frac{1}{4}}$ is an empirical correction for. the dependence of . the density of the gas in the boundary layer on temperature. 


$$
r=r\left(x_{r}\right)
$$

Equation $(3-46)$ is the basic expression that was employed in the subject investigation to correlate the roughness parameter $r$. The value of the roughness parameter $r$ was estimated for each experimental test as follows:

(a) The average value for the simple rate of mass transfer, $\mathrm{m}_{\mathrm{s}}$, was calculated following the procedure outlined in subsection 3.1.2.

(b) The value of the entrainment parameter $e_{0}$ was determined from the average value of the entrainment group $x_{e}$ for that test, together with the analytical expression given by Eq. $(3-32)$.

(c) The value of the intercept "A" (see Fig. 19) was estimated by determining which curve having the slope (1-e $)$ resulted in the best "eyeball" fit of the experimental data. (d) The roughness parameter $r$ was then determined from the expression

$$
r=\frac{A}{m_{s}^{T}}-1.0
$$

Figure 22 presents the roucinness parameter $r$--determined by the aforementioned procedure--as a function of the parameter $x_{r}$. The various conditions for the gas stream pressure and temperature are listed in the figure. Although the data 


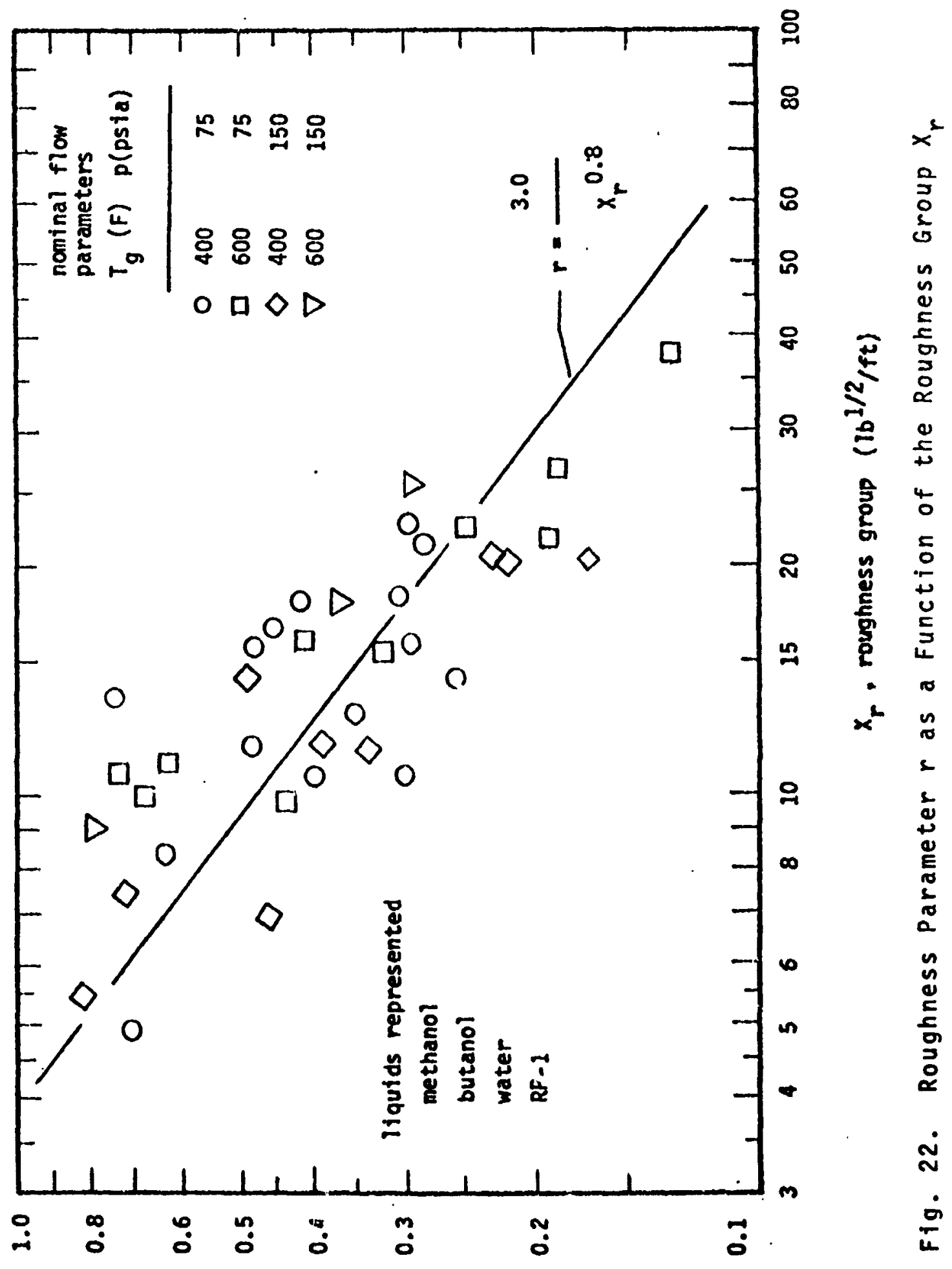

dajaured ssauybnod 'A 
in Fig. 22 do exhibit some scatter, much of the scatter can be attributed to the fact that the value for the intercept A was very sensitive to relatively small errors in the measurement of the net rate of mass transfer from the liquid film to the gas stream. Furthermore, any error in determining the value of the intercept $A$ was compounded in the calculation of the roughness parameter $r$. To illustrate, assume that the true value for the parameter $A / \mathrm{m}_{\mathrm{s}}^{\prime}$ is 1.2 and that the experimentally determinec :ulue is 1.4. From Eq. $(3-47)$, the corresponding values for the roughness parameter $r$ are 0.2 and 0.4 , respectively. Thus, for this example, an error of approximately 17 percent in the intercept $A$ results in an error of $5 u$ percent in the evaluation of $r$. In addition to that problem, some of the scatter of the data in Fig. 22 is probably due to the fact that the rather complex phenomenon of interfacial roughness cannot be characterized completely in terms of the relatively simple parameter $x_{r}$. However, considering the wide range of values for the liquid and the gas flow parameters that is represented by the data in Fig. 22 , the correlation of $r$ in terms of the group $x_{r}$ would appear to have some physical justification.

The solid curve that is shown in Fig. 22 is given by the following analytical expression. Thus

$$
r=\frac{3.0}{x_{r} 0.8}
$$


where $x_{r}$ has dimensions of $1 b_{f}^{\frac{1}{2}} / f t$. While the experimental data in Fig. 22 are in general agreement with this analytical expression, the final form of Eq. (3-48) was arrived at by considering the following three points, which are listed in their order of importance:

(a) The correlation of all of the experimental data of the present investigation, including those for which the gas stream was accelerated over the wetted test plate;

(b) The correlation of the mass transfer data that were reported by Kinney, Abramson, and Sloop (19) (these data are analyzed in Section 4.3); and

(c) The correlation of the experimental data for mass transfer that are reported by Emmons (20) (these data are analyzed in Section 4.4).

It is important to note that Eq. $(3-48)$ and the data in Fig. 22 suggest that the interfacial roughness can contribute substantially to the convective transport of energy from the hot gas stream to the surface of the liquid film. Indeed, Fig. 22 suggests that the simple mass transfer rate $m_{s}^{\prime}$ could be increased hy 100 percent $(r=1)$, or more, due to the interfacial roughiess. In comparison, for the case of heat transfer to a dry rough wall, Fig. 7-56 of Ref. (39) shows that the value for $r$ can be as great as 2.5. It would seem, therefore, that a value of $r$ of 1.0 for the problem of liquid-film cooling is not unreasonable. It should be emphasized, however, that in the analysis of the experimental 
data of the presert investigation and in the analysis of the data of the primary references (19) and (20), that the contribution to the net mass transfer rate that was due to the phenomenon of interfacial roughness was, with very few exceptions, substantially less than the contribution that was due to the entrainment phenomenon. Frequently, the mass transfer due to entrainment was 10 to 20 times the evaporative mass transfer that was attributed to the effective roughness of the surface of the liquid film.

\subsubsection{The comparison of the Predicted and} the Measured Rates of Mass Transfer

A comparison is presented in this subsection of the rates of mass transfer from the liquid film to a constant velocity gas stream that were predicted from the correlation developed herein to those that were determined experimentally. The comparison is presented for both the datum points that were obtained for the hot-flow experiments (approximately 500 datum points) and those that were obtained for the coldflow experiments ( 76 datum points). In addition, to emphasize the utility of the present theory, the experimental data are also compared with the results that were predicted from the simple theory of mass transfer. The latter comparison serves to illustrate graphically the general inadequacy of the simple theory for the subject problem.

The primary equation that was employed to calculate the rates of mass transfer from the liquid film to the gas stream is repeated here for convenience. Thus 


$$
m^{\prime}=(1+r) m_{s}^{\prime}+e_{0} m_{1}
$$

The simple mass transfer $m_{s}^{\prime}$ was calculated following the cievelopments of subsection 3.1.2. The roughness parameter $r$ was calculated from Eq. (3-48) and the e.strainment parameter e was determined from E.q. (3-32). The surface tension 0 that appears in the entrainment group $X_{e}$ in $E_{1} \cdot(3-32)$ was based on the average of the temperature of the liquid at the point of injection and at the point of withdrawa ${ }^{*}$. All of the numerical computations were performed on an IBil 7094 digital computer.

Figures 23 through 26 summarize graphically the results of the aforementioned calculations for those experimental data where the gas stream was heated. Each figure represents one of the four liquids that was investigated (Fig. 23: methanol; Fig. 24: butanol; Fig. 25: water; Fig. 26: RP-1). The nominal value; for the gas stream pressure and the gas stream temperature for each set of experimental data are indicated in the figures. Part (a) of each figure is a plot of the simple mass transfer rate, $m_{s}^{\prime}$, that was predicted from the theory outlined in subsection 3.1 .2 versus the measured rate of mass transfer. Part (b) of each figure compares the measured rate of mass transfer with the rate of mass transfer that was predicted from

* See Table G.I of Appendix $G$ for a tabulation of the average inlet and outlet liquid temperatures for. each experinental. test. 


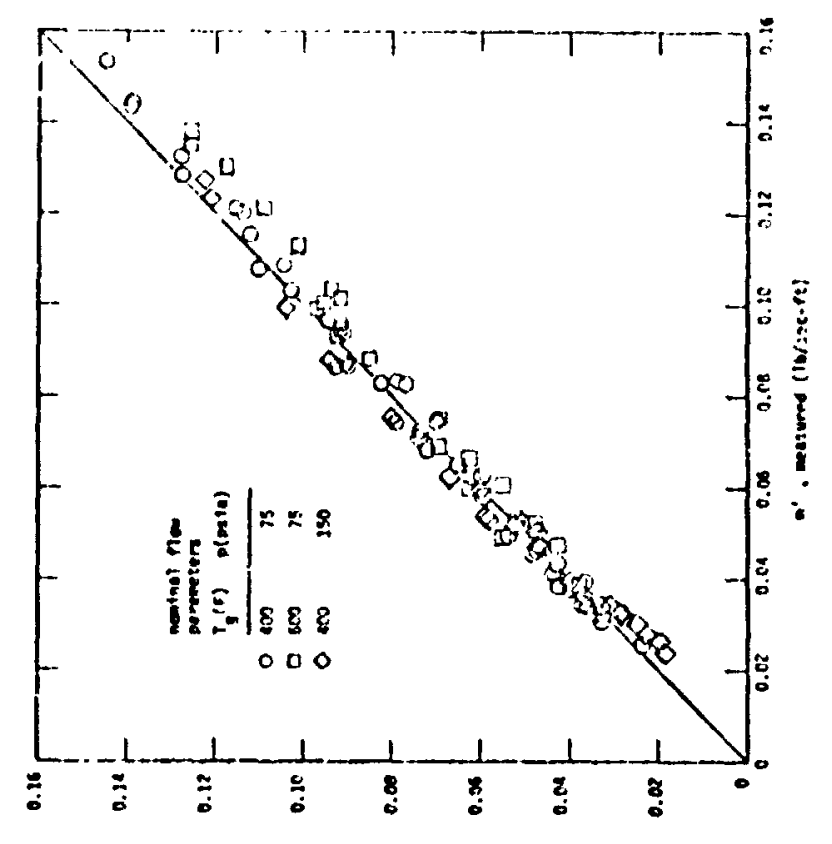

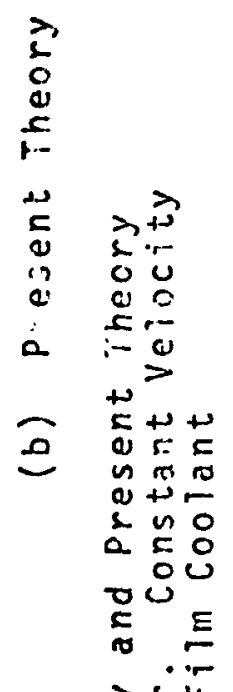

$\rightarrow$ s. 11.

10

is is

$\therefore \frac{5}{2}$

0.5

$\ln \stackrel{5}{=}$

$\underset{\pi}{n}$

2. E

000

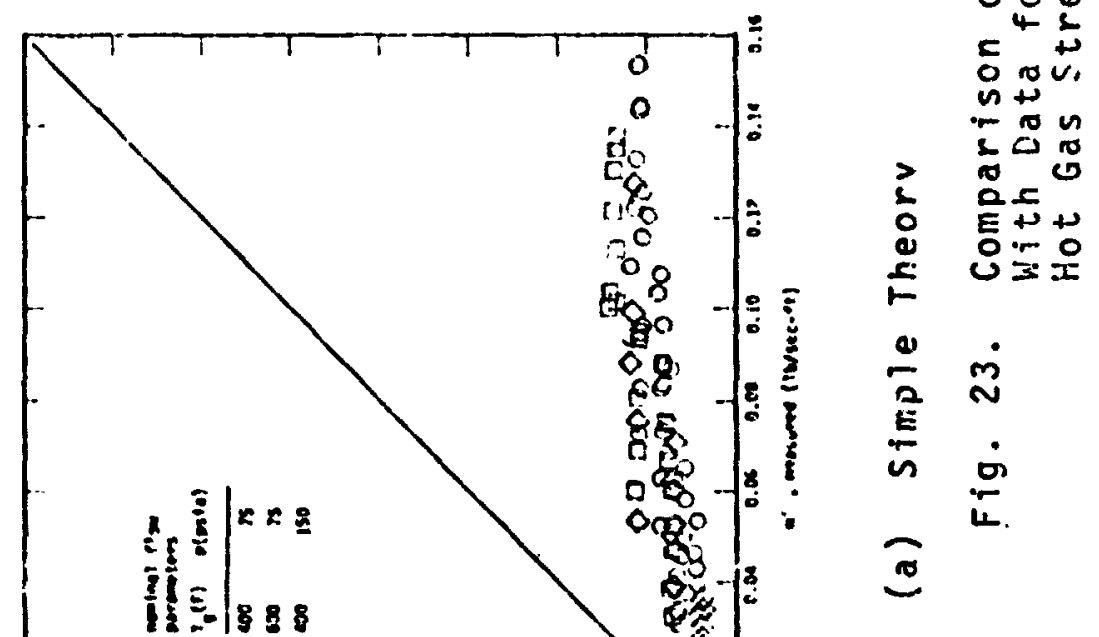


5

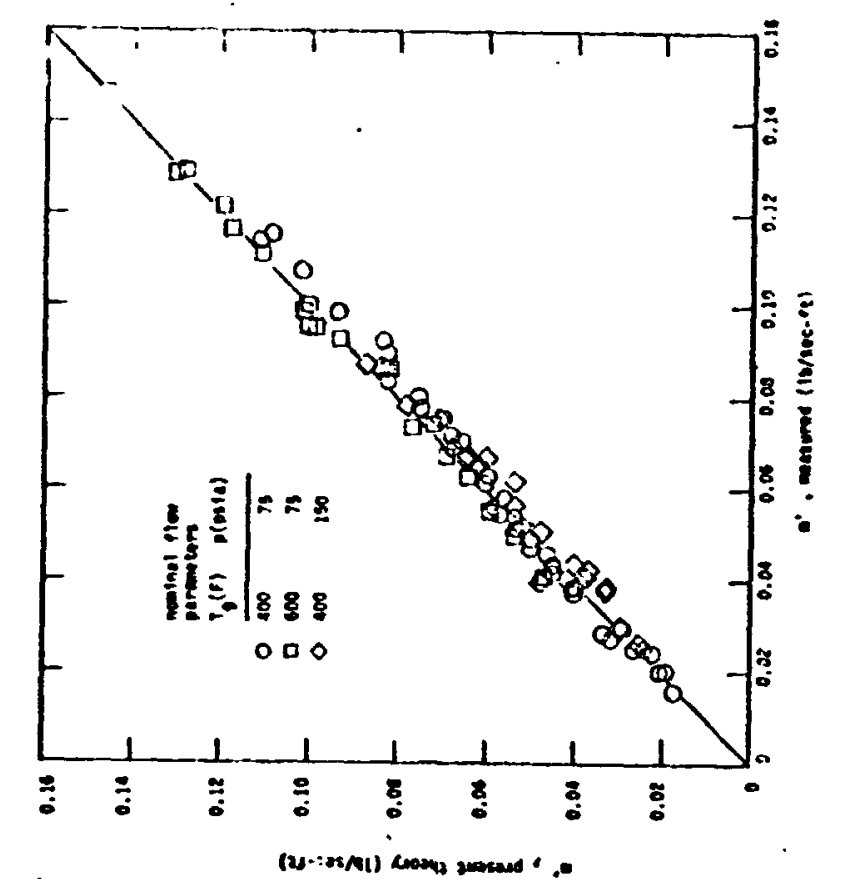

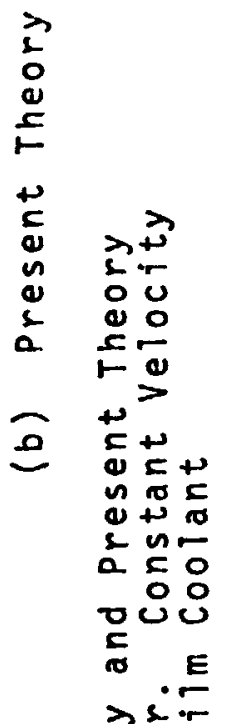

क्षे

04

\&

5 死

2

車

En

E

U $\Sigma$

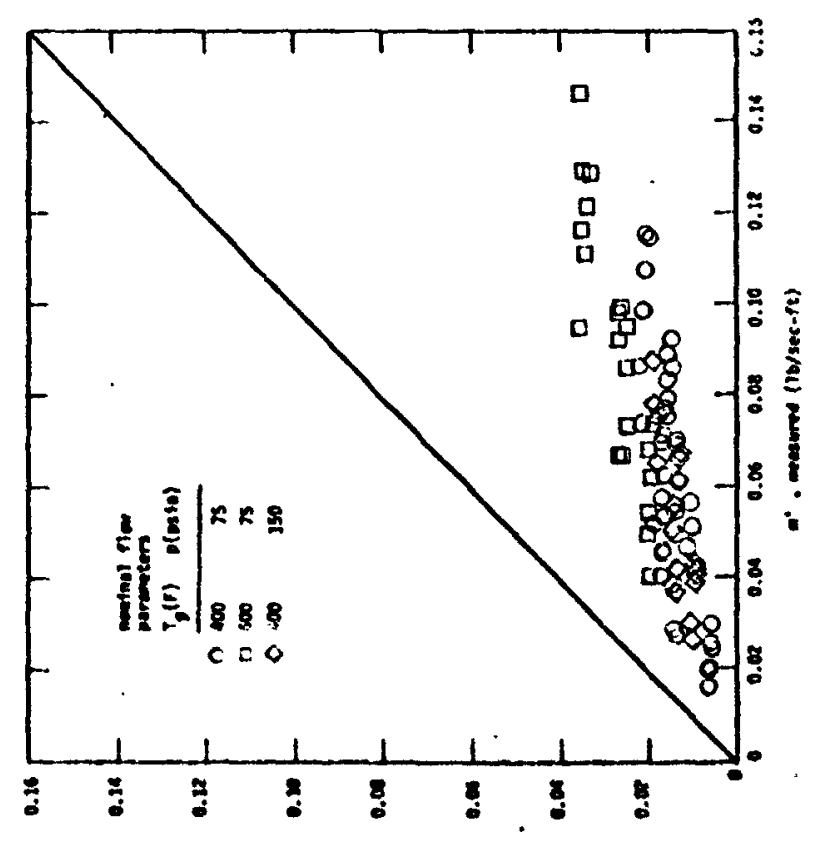

425

ㅇㅇㄴ

c。苔

品

-

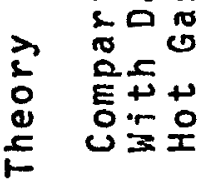

8

0

0

8

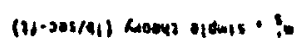

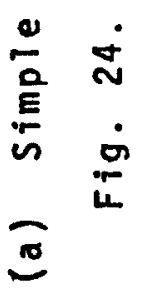



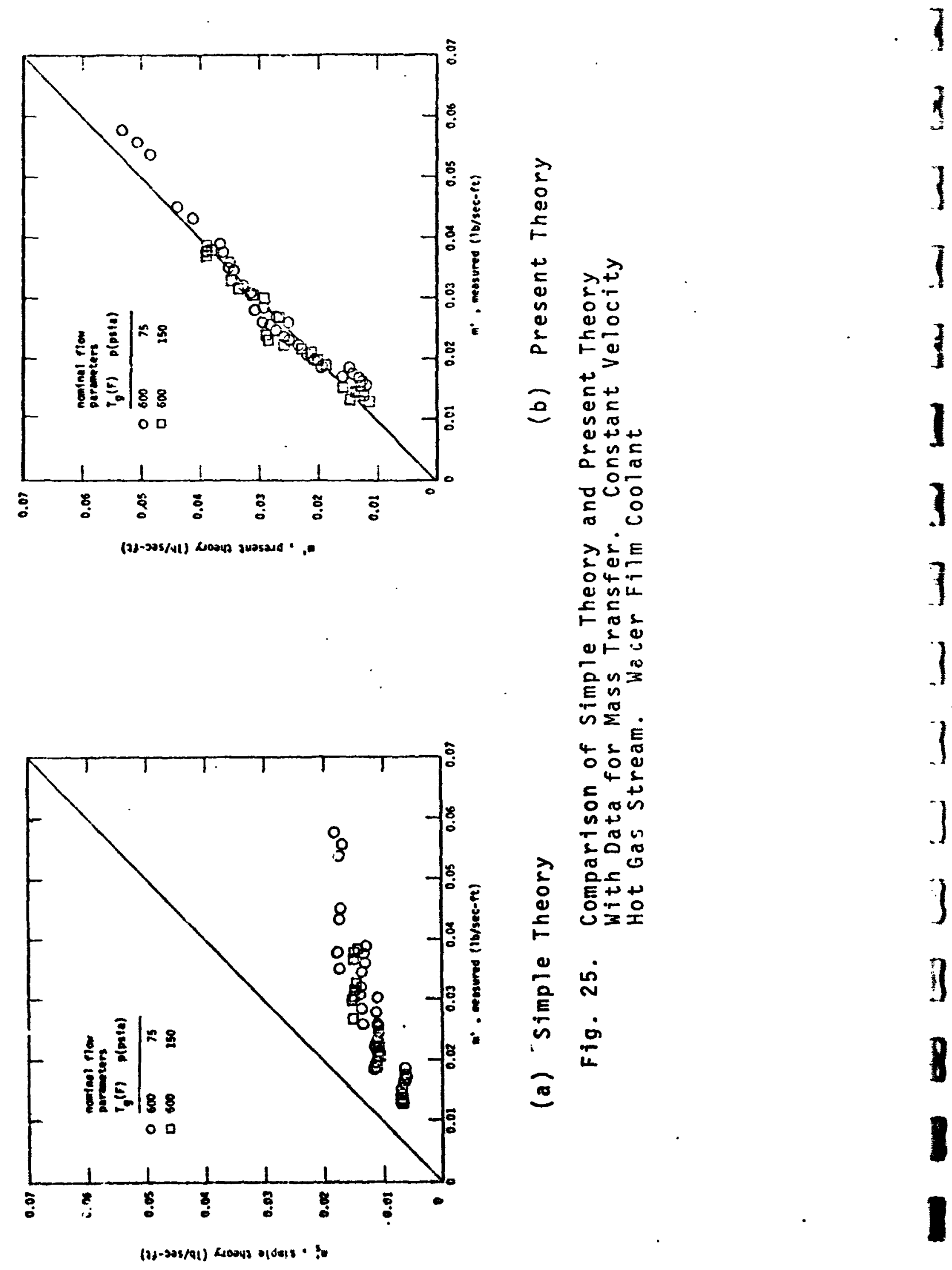

1

1

] 은

$+$

点

党尔

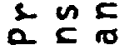

잉ㅇㅇ

$\rightarrow$ i

रो:

व w口

占造

ข동

两 $n 3$

品

is

E

- 인 


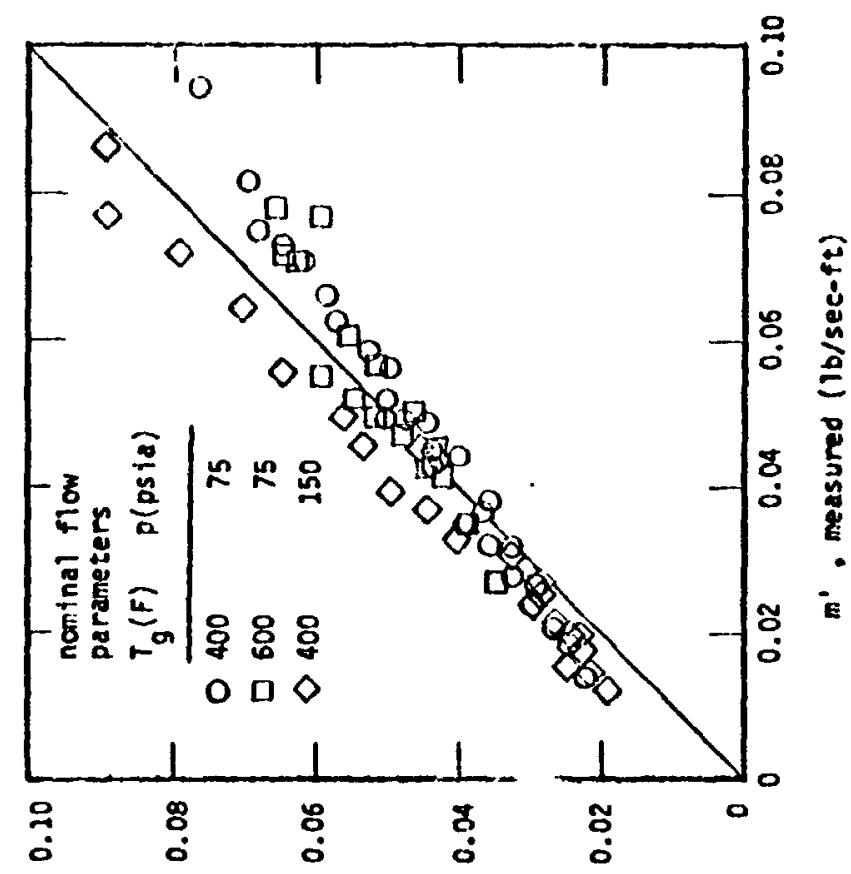

(2j-3as/91) Racayz guasadd " ,

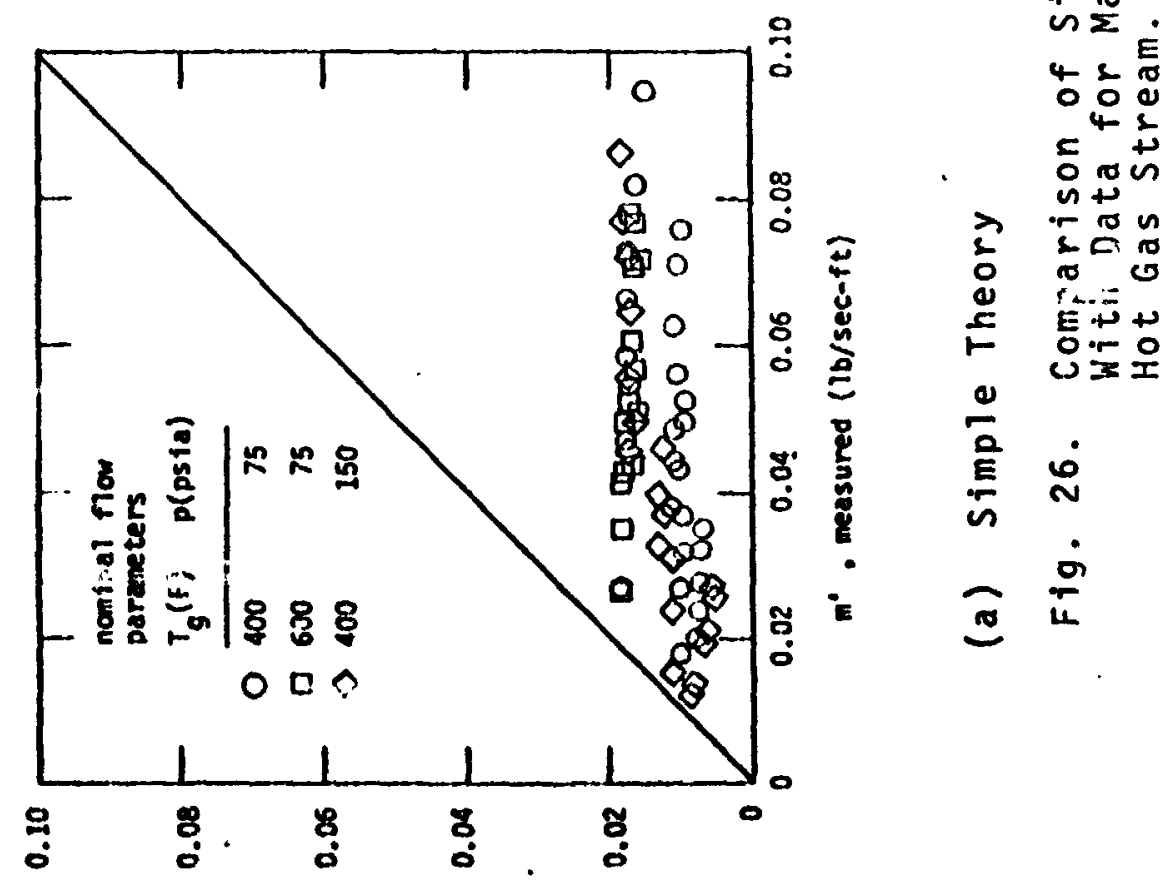

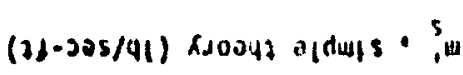


Eq. (3-6) for the present theory. Each figure graphically demonstrates that, in general, the simple thecry for mass transfer is a poor approximation to the complex phenomena that characterize liquid-film cooling. The maximum difference between the experimental result and the result that was predicted from the simple theory is shown in Fig. 23(a) for methanol as the film coolant. For that figure, the maximum value for the measured mass transfer rate is $0.154 \mathrm{lb} / \mathrm{sec}-\mathrm{ft}$ and the corresponding simple rate of mass transfer is 0.022 $1 b / s \in c-f t$, a difference of 700 percent. The present theory, however, accounts for the existence of the interfacial phenomena, and the resultant agreement between the predicted and the measured mass transfer rates that is shown in part (b) of each figure is quite satisfactory. The maximum difference between the result that is predicted from the present theory and the experimental result is shown in Fig. 26(b) for RP-1 as the film coolant. For a measured rate of mass transfer of $0.0944 \mathrm{lb} / \mathrm{sec}-\mathrm{ft}$ in that figure, the corresponding mass transfer rate that is predicted by the present theory is $0.0765 \mathrm{lb} / \mathrm{sec}-\mathrm{ft}$, a difference of about 20 percent.

Figure 27 presents the results of the computations that were performed for the case where the gas stream was not heated. Presented in the figure is a plot of the mass transfer rate that was predicted by the present theory versus the measured rate of mass transfer. The experimental data in the figurs are for one nominal gas stream pressure of 75 psia and for the liquids methanol, butanol, 


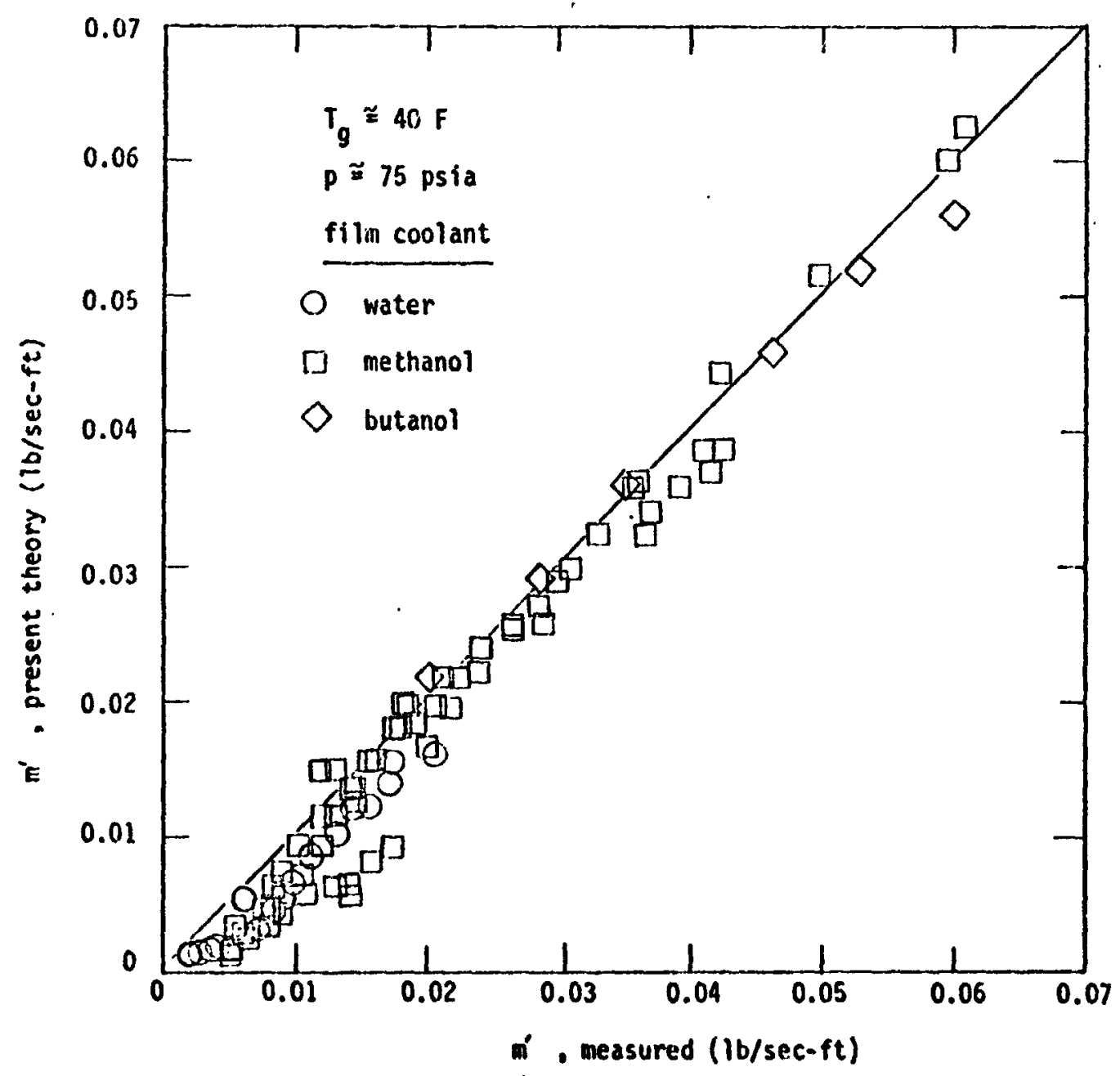

Fig. 27. Comparison of Present Theory With Data for Mass Transfer. Constant Velocity Cold Gas Stream 
and water*. It was assumed in the computations that the rate of simple mass transfer, $m_{s}^{\prime}$, was essentially zero because of the low gas stream temperatures. Thus, the realized rates of mass transfer were assumed to be due to entrainment only. The masimum value that was computed for the entrainment parameier $e_{0}$ was 0.32 , as compared to a maximum value of 0.63 for the hot-flow tests. The agreement between the measured and the predicted rates of mass transfer is quite satisfactory, particularly in view of the fact that the pertinent physical properties for both the liquid and the gas phases differed so significantly between the case where the gas stream was heated and the case where it was not. For example, the viscosity for the liquid varied by as much as a factor of 15:1, the surface tension for the liquid by as much as a factor of $1.5: 1$, and the viscosity for the gas stream by as much as a factor of $2: 1$ between the hot and cold gas stream flow conditions. It would appear, therefore, that the relatively simple theory that is proposed herein for determining the rate of mass transfer from a thin liquid film to a high velocity gas stream can be applied quite generally. The utility of the present theory is demonstrated further in section 4 of this report where the correlation of

\footnotetext{
* The number of datum points that could be obtained with water as the film coolant $W=$ ? i...ited because the gas stream was generally cooled sufficientiy by the expansion from the storage tank pressure of 1000 to 2500 psia to the test section pressure cf 75 psia that a portion of the water that was injected onto the test plate froze before it reached the capture siot. Morec:er, no exnerimenta? tests vere performed with ip-l as thi liquici phase because of the limited supply of this liguid.
} 
the experimental data for liquid-film cooling of the primary references $(19,20,24)$ is considered. The gas stream flow conditions that characterized those investigations will be shown to be, in most instances, much more severe than those that characterized the present investigation.

The analyses of the experimental data of the aforementioned primary references requires first the consideration of the problem of how the basic correlation for mass transfer can be extended to the more general case where the film cooled length 1 is arbitrary. That is, the case where 1 does not necessarily equal $1_{0}=10$ inches. The following section presents an analysis of that problem. The result of the analysis will also suggest a method for correlating the experimentai data for mass transfer that were presented in Section 2.6 of the present report for the case where the gas stream was accelerated over the film cooled length $1_{0}$. The correlation of those data is then discussed in Section 3.3.

\subsection{The Modification of the Basic Correlation for $\frac{\text { far Mass Transfer to Account }}{\text { an Arbitrary film Cooled length }}$}

The correlation that was developed for the entrainment parameter $e_{0}$ in Subsection 3.1 .3 is necessarily dependent on $1_{0}$, the length of the film cooled test section for the subject investigatiull. That is. if the film cooitd length $1_{0}$ had been, say, greater than the 10-in. length that was employed, then, for the same flow conditions, there would have been more entrainment of liquid into the gas stream because 
of the prolonged exposure of the surface of the liquid film to the gas stream shear forces. In order to be able to predict the llass transfer that occurs for an arbitrary film cooled length 1 , the basic correlation for $e_{0}$ has to be modified. A rather simple model and analysis is developed in subsection 3.2 .1 to solve that problem. Subsection 3.2 .2 then suggests an empirical approximation to the result of that analysis that is generally more convenient to employ.

\subsubsection{Model and Analysis}

Figure 28 presents the simple model that is employed in the analysis. The pertinent notation for the figure is defined as follows:

$$
\begin{aligned}
x^{\prime} & =x-x_{1} \\
m_{1} & =\text { the rate of liquid injection at } x^{\prime}=0 \\
1 & =\text { the liquid-film cooled length } \\
l_{0} & =\text { the length of each film element* } \\
n & =1 / l_{0} \text { (assumed to be an integer) } \\
m_{k}^{\prime} & =\text { the net rate of mass transfer from the } k \text { th } \\
m_{s, k} & =\text { the net rate of simple mass transfer for the } \\
& \text { kth film element, } k=1,2,3, \ldots . n
\end{aligned}
$$

* For the purposes of the present analysis, it is not necessary to specify that ${ }_{0}=10$ inches. However tn itiilze the result of the analysis to perform any numerical calculations, it will be necessary to make that specification. 

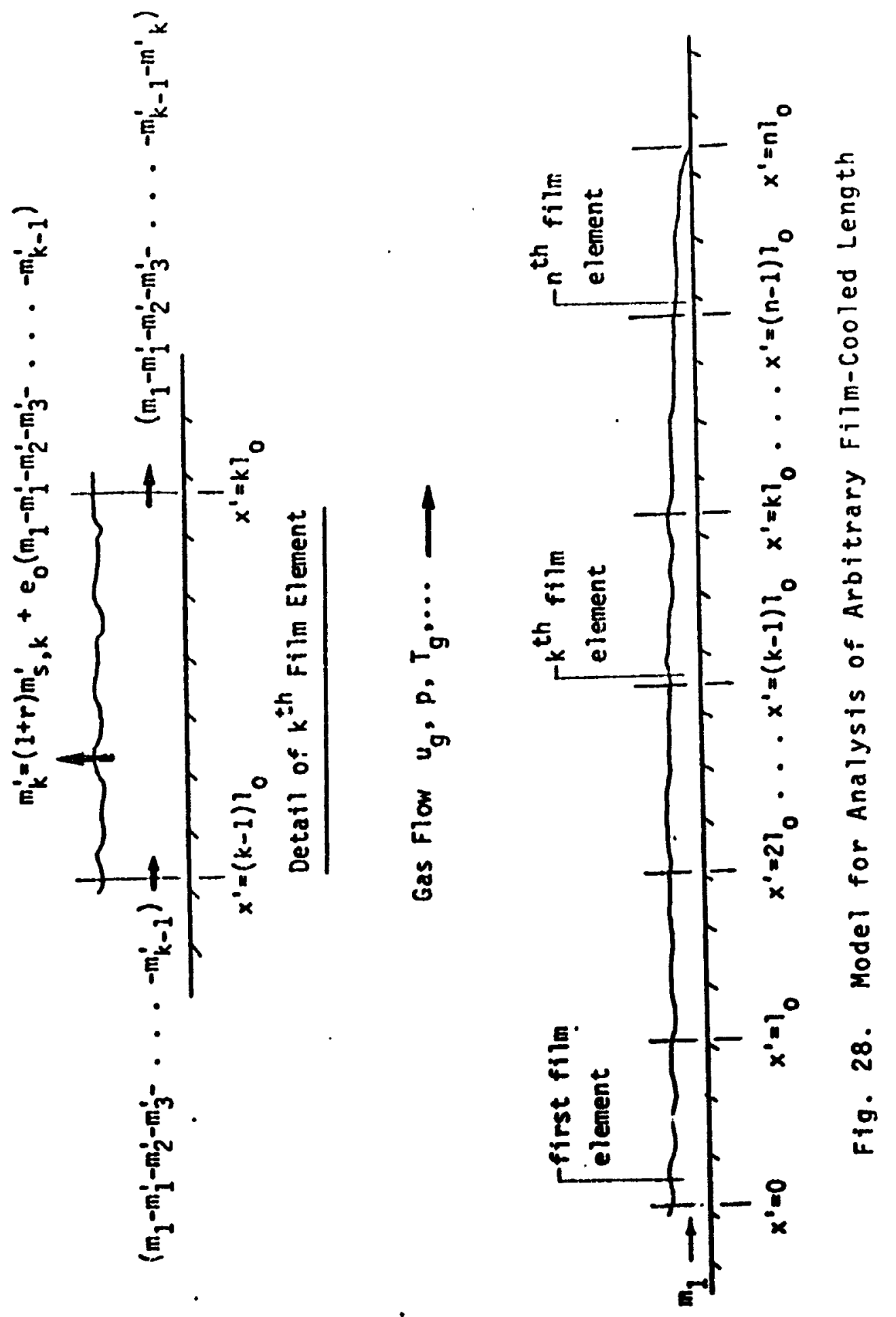
The basic assumptions employed in the analys is are as follow:

(a) The flow parameters for the gas stream are independent of $x^{\prime}$.

(b) The roughness parameter $r$ is independent of $x^{\prime}$.

(c) The local ratc of simple mass transfer varies with the coordinate $x^{\prime}$ as

$$
m_{s}^{\prime \prime}=c x^{\prime p}
$$

where $c$ and $p$ are appropriate constants.

(d) The primary equation (3-6) applies to each of the elements of the liquid film.

(e) The liquid film terminates when $1=n 1_{0}$, where $n$ is an integer.

Thus, for the first element of the liquid film, the net rate of mass transfer is given by

$$
m_{1}^{\prime}=(1+r) m_{s, 1}^{\prime}+e_{0}\left(m_{1}\right)
$$

Similarily, the mass transfer for the second film element is given by.

$$
m_{2}^{\prime}=(1+r) m_{s, 2}^{\prime}+e_{0}\left(m_{1}-m_{1}^{\prime}\right)
$$

In general, for the kth element of the liquid film, the net 
rate of mass transfer $m_{k}^{\prime}$ is expressed as

$$
m_{k}^{\prime}=(1+r) m_{s, k}^{\prime}+e_{0}\left(m_{1}-m_{1}^{\prime}-m_{2}^{\prime}-\ldots-m_{k-1}^{\prime}\right)
$$

Suistitution of Eq. (3-51) into Eq. (3-52) gives the result

$$
m_{2}^{\prime}=(1+r) m_{s, 2}^{\prime}+e_{0}\left[m_{1}-(1+r) m_{s, 1}^{\prime}-e_{0} m_{1}\right]
$$

Substitution of Eqs. (3-51) and (3-54) into Eq. (3-53) with $k=3$ yields

$$
\begin{aligned}
& m_{3}^{\prime}=(1+r) m_{s, 3}^{\prime} e_{0}\left\{m_{1}-(1+r) m_{s, 1}^{\prime}-\right. \\
& e_{0} m_{1}-(1+r) m_{s, 2}^{\prime}-e_{0}\left[m_{1}-(1+r) m_{s, 1}^{\prime}-\right. \\
& \left.\left.e_{0} m_{1}\right]\right\}
\end{aligned}
$$

This substitution procedure can be continued in a stepwise fashion so that for the general kth term $m_{k}$, the terms $m_{1}$, $m_{2}^{\prime}, \ldots m_{k-1}^{i}$ can be eliminated from the resultant expression. Moreover, after considerable algebra and a fortuitous combining of terms, it is possible to show that

$$
\sum_{k=1}^{n} m_{k}^{\prime}=m_{1}-m_{1}\left(1-e_{0}\right)^{n}+(1+r) \sum_{k=1}^{n} m_{s, k}^{\prime}\left(1-e_{0}\right)^{n-k}
$$


But, if it is assumed that the iqquid in the film is consumed at a point where $1=n 1_{0}$, then it follows from the conservation of the liquid that

$$
\sum_{k=1}^{n} m_{k}^{\prime}=m_{1}
$$

Equation (3-56), therefore, can be rewritten in the form

$$
m_{1}=(1+r) \sum_{k=1}^{n} m_{s, k}^{\prime}\left(1-e_{0}\right)^{-k}
$$

From assumption (c) of the model it follows that the rate of simple mass transfer from the kth film element is given by

$$
m_{s, k}^{\prime}=\int_{(k-1) 1_{0}}^{k 1_{0}} m_{s}^{\prime \prime} d x^{\prime}=c \frac{\left.\left\{(k)_{0}\right)^{p+1}-\left[(k-1) 1_{0}\right]^{p+1}\right\}}{(p+1)}
$$

Moreover, the total rate of simple mass transfer from the liquid film is equal to

$$
m_{s}^{\prime}=\int_{0}^{n 1} m_{s}^{\prime \prime} d x^{\prime}=c \frac{\left.(n)_{0}\right)^{p+1}}{(p+1)}
$$


Finally, substituting Eq. (3-59) into Eq. (3-58) and dividing through both sides of the resultant expression by Eq. (3-60) yields the following non-dimensional equation. Thus

$$
\frac{m_{1}}{m_{s}^{1}}=(1+r) \sum_{k=1}^{n}\left[\left(\frac{k}{n}\right)^{p+1}-\left(\frac{k-1}{n}\right)^{p+1}\right]\left(1-e_{0}\right)^{-k}
$$

Equation $(3-61)$ is the desired analytical result. For given gas stream and liquid flow conditions (and, therefore, given values for the parameters $p, r$ and $\left.e_{0}\right)$, Eq. (3-61) can be utilized to calculate the net rate of mass transfer from the liquid film into a constant velocity gas stream if the film cooled length 1 is equal to $n 1_{0}$, where $n$ is an integer. The obvious disadrantage of Eq. $(3-61)$ is the fact that it can be utilized for only those special cases where $1=n 1_{0}$. Therefore, it is convenient to replace that analytical result by an approximate empirical expression that is a continuous function of 1 , where 1 can be either greater than or less than $1_{0}$. Such an empirical expression is developed in the following subsection.

* Note that for the special case where the interfacial phenomena are not important, i.e., $r=e_{0}=0$, then the RHS of Eq. (3-62) reduces to unity as required. 
3.2.2 An Empirical Approximation to the Analytical Result

An empirical approximation to the analytical equation (3-51) can be derived as follows. First note that for the special case where $1=1_{0}$. Eq. $(3-61)$ reduces to the simple expression

$$
\frac{m_{1}}{m_{s}^{1}}=(1+r)\left(1-e_{0}\right)^{-1}
$$

Now assume that Eq. (3-62) can be modified as follows for the case where the film cooled length is arbitrary. Thus

$$
\frac{m_{1}}{m_{s}^{1}}=(1+r)\left(1-e_{0}\right)^{-\left(1 / 1_{0}\right)^{b}}
$$

The parameter $b$ in Eq. $(3-63)$ is determined by first specifying a value for the parameter $p$ that appears in the analytical expression (3-61), and then "matching" the empirical expression (3.63) and the analytical expression (3-61) at those points where $\left(1 / 1_{0}\right)=$ an integer.

To illustrate the aforementioned procedure for the evaluation of the parameter $b$, consider the classical case of the turbulent transport of heat and mass from a flat plate where the parameter $p$ in Eq. (3-50) is equal to $-1 / 5$. Figure 29 presents a plut of the quantity $\left(m_{1} / m_{s}^{\prime}\right) /(1+r)$ as a function of the ratio $\left(1 / 1_{0}\right)$ with the entrainment parameter $e_{0}$ as a parameter. The solid curves shown in Fig. 29. 


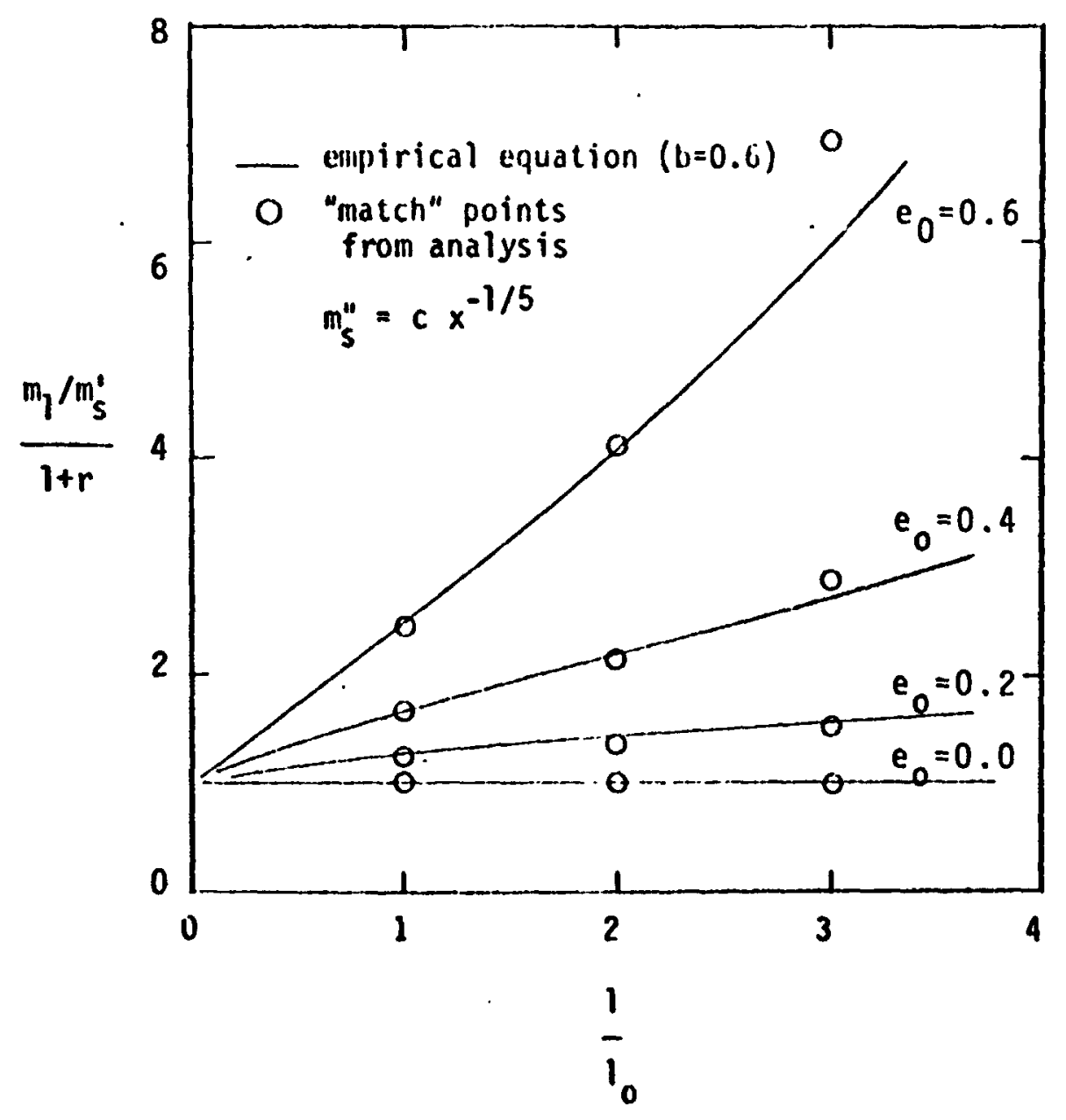

Fig. 29. Matching of Empirical Equation to Analytical Result

$$
m_{s}^{\prime \prime}=c x^{-1 / 5}
$$


were calculated from Eq. (3-63) with a value for $b$ of 0.6 , and the circles are the "match" points that were calculated from the analytical expression $(3-61)$.

Figure 30 presents another illustrative example for the case where the parameter $p$ in $E q .(3-50)$ is equal to 1.0 ; that is, the case where the rate of simple mass transfer increases linearly with the coordinate $x^{\prime *}$. The solid curves were calculated from Eq. (3-63) where the value of 0.9 for the parameter $b$ was employed, and the "match" points were calculated from Eq. (3-61).

Figures 29 and 30 show that the analytical result of Subsection 3.2.1 can be approximated with sufficient accuracy by the relatively simple empirical formula of the present section. Moreover, these figures show that a substantial change in the parameter $p$ in Eq. (3-61) requires a relatively small change in the parameter $b$ in the empirical expression (3-63) in order to obtain an adequate matching of the analytical and empirical results. form. Substituting the analytical expression (3-48) for the roughness parameter $r$ and the analytical expression (3-32) for the entrainment Darameter $e_{0}$. Eq. (3-63) can be
- The significance of the foregoing developments can be further illustrated by writing Eq. (3-63) in a more explicit significance. 


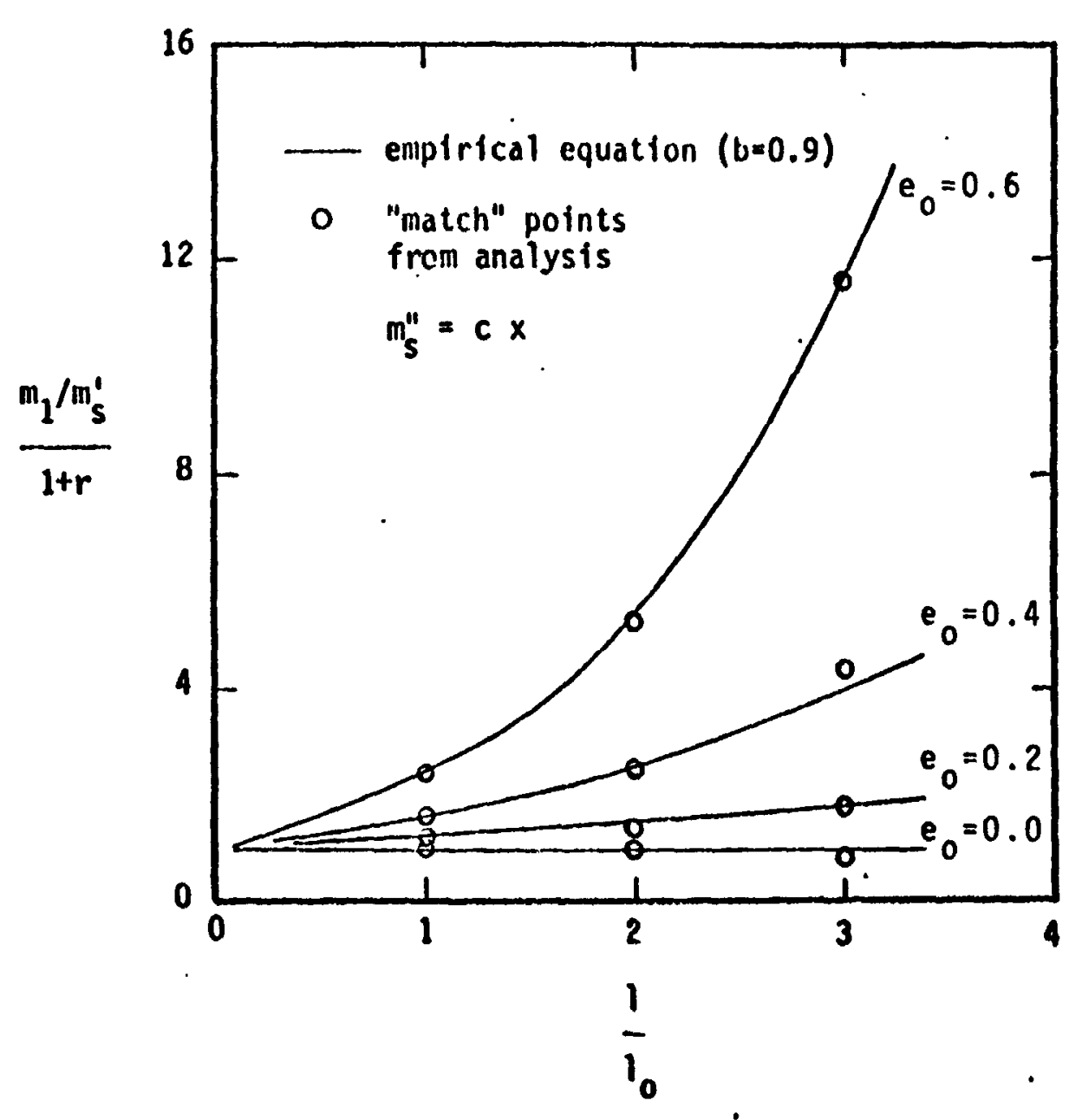

Fig. 30. Matching of Empirical Equation to Analytical Result $m_{s}^{\prime \prime}=c x$ 
written in the form

$$
\frac{m_{1}}{m_{s}^{1}}=\left(1+\frac{3.0}{x_{r}^{0.8}}\right) \text { exp }\left[5 \times 10^{-5}\left(x_{e}-1000\right)\left(1 / 1_{0}\right)^{b}\right]
$$

Thus, if the value for the parameter $b$ is approximated from the aforementioned "matching" procedure, Eq. (3-64) gives an explicit expression that can be employed to calculate the net rate of mass transfer from a liquid film of arbitrary length 1 to a constant velocity gas stream.

The validity of the foregoing analysis (and, therefore, Eq. (3-64)) will be investigated in Section 4 of the present report. Considered in that section is the correlation of the experimental data for mass transfer that were reported in the primary references $(19,20)$. The range of values for the film cooled length 1 that is represented by those data is 3 inches to 36 inches long.

Equation (3-64) also serves to suggest a method for the correlation of the experimental data for mass iransfer that were obtained for the case where the gas stream was accelerated

* So that the reader is not confused, the substitution of Eq. (3-32) for the entrainment parameter $e_{0}$ requires that the length $1_{0}$ in Eq. (3-64) be taken as 10 inches. Moreover, the numerical constants in Eq. (3-64) require that the roughness group $x_{r}$ be substituted with the units $\left(1 b_{f}^{\frac{1}{2}} / f t\right)$ - and the entrainment group $x_{e}$ be substituted with the units $\left(1 b_{f}^{-\frac{1}{2}}\right)$. 
over the film cooled length $1_{0}$. Those data were presented in section 2.6 of the present report. The correlation of the subject data is considered in the following section.

\subsection{Correlation of the Data for Mass Transfer for an Accelerating Gas Stroam}

The purpose of the present section is to correlate the experimental data for mass transfer that were obtained for the case where the gas stream was accelerated over the length of the film cooled test plate. These data were shown graphically in Fig. 17 of Section 2.6. Subsection 3.3.1, which follows, shows how the simple rate of mass transfer was computed for the subject case. Subsection 3.3 .2 then demonstrates how that simple rate of mass transfer was employed, together with a logical extension of the developments that were presented in sections 3.1 , and 3.2 , to correlate the experimental data for an accelerating gas stream. The comparison between the experimental and the predicted net mass transfer rates is presented in subsection 3.3.3.

\subsubsection{The Evaluation of the Simple Rate of} Mass Transfer for an Accelerating Gas Flow

Kays (36) suggests that the following expression can be employed to evaluate the local rate of simple mass transfer for the case where the gas stream is accelerating. Thus 


$$
m_{s}^{\prime \prime}=G B_{h} F_{T} F_{C} F_{m} F_{P r}\left[0.0295 \operatorname{Re}_{x}^{1-.08} \operatorname{Re}_{x_{t}^{\prime}}^{-0.12}\right]
$$

where

$$
\begin{aligned}
& \operatorname{Re}_{x}^{\prime}=\frac{1}{\mu_{g}} \int_{0}^{x} G d x \\
& \operatorname{Re}_{x_{t}}^{\prime}=\frac{1}{\mu_{g}} \int_{x_{t}}^{x} G d x
\end{aligned}
$$

The " $F$ " correction factors in Eq. (3-65) are given by Eqs. $(3-18)-(3-21)$ of subsection 3.1.2. For the purposes of the present calculations, the energy balance parameter $\phi$ that enters into the definition of the parameter $B_{h}{ }^{* *}$ was approximated from Eq. $(3-13)$ of Subsection 3.1.2. Moreover, because of the 1 imited Mach number range that was investigated, the variation in the gas stream temperature, $T_{g}$, over the film cooled length $1_{0}$ was so small that the average of

Kays (36) introduces an additional empirical correction factor into Eq. $(3-64)$ to correct for acceleration effects that are nat predisted analytically. However, preliminary calculation of that correction factor showed that it was $\therefore$ the order of 0.98 for the range of flow cuncilions for the subject investigation. Therefore, that correction factor was not considered in the present calculations for $\therefore m_{s}^{\prime \prime}$.

**.See Eq. (3-22). 
the values of $T_{g}$ at $x=x_{1}$ and at $x=x_{2}$ was employed to calculate the parameters $\mu_{g}, B_{h}$, and $F_{T}$ in Eq. (3-65). To complete the solution for $m_{s}^{\prime \prime}$, it is necessary to determine the local values for the static pressure $p$, the gus stream Mach number $M_{a}$, the gas stream velocity $u_{g}$, and the mass velocity for the gas stream $G$. The procedure employed to evaluate those parameters is outlined as follows. The static pressure was measured at the location $x=20$ inches in the approach section and at 5 axial locations (equispaced at 2 inches) in the test section by wall taps that were located in the roof of the experimental tunnel. The stagnation pressure for the gas. flow was calculated from the static pressure that was measured in the approach section, together with the value that was computed for the gas stream velocity at the entrance to the test section*. The local value for the Mach number within the experimental test section was computed by forming the ratio of the measured static pressure and the calculated stagnation pressure, and then employing the following relationship for isentropic flow with a specific heat ratio $Y=1.385$. Thus.

$$
\frac{p}{p^{0}}=11+\frac{r-1}{2} m_{a}^{2} !^{\frac{r}{r-1}}
$$

* See Append $1 x$ F.2 for the procedure that was employed to calculate the gas stream velocity at the entrance to the test section. 
The complete profile for the Mach number throughout the test section was obtained by fitting a third-order polynominal to the local vallies for the Mach number that were computed from the experimentai measurements for the static pressure. The resultant form or the approximate expression for the Mach number as a function of $x^{\prime}$, the distance from the point of liquid injection, was

$$
M_{a}=0.145+0.02 x^{\prime}-0.0014 x^{12}+0.00028 x^{13}
$$

The local values for the gas stream velocity, $u_{g}$, were computed from Eq. (3-69) and the equation for the acoustic speed in a perfect gas:

$$
u_{g}=m_{a}\left(\gamma R_{g} T_{g}\right)^{0.5}
$$

Finally, the local values for the gas mass velocity, $G$, were calculated from the defining equation

$$
G=\rho_{g} u_{g}
$$

where the gas stream density, $\rho_{g}$, was evaluated from the equation of state for a perfect gas:

$$
P_{g}=\frac{p}{R_{g} T_{g}}
$$


Figure 31 shows graphically the values that were computed from the foregoing procedure for the parameters $p / p^{0}, M_{a}$, and $u_{g}$ as a function of $x^{\prime}$. The solid curves that are presented in the figure are based on the analytical expression (3-69) for the Mach number. The circles presented in Fig. 31 represent the static-stagnation pressure ratios that were realized experimentally. Each circle presented in the figure represents the approximate range of values for $\left(p / p^{0}\right)$ that was obtained at that parlicular location for the entire spectrum of gas flow conditions that was investigated.

The foregoing equations were programmed for an IBM 7094 digital computer and the integral expressions for the terms $\operatorname{Re}_{x}^{\prime}$ and $\operatorname{Re}_{x t}^{\prime}$ were evaluated numericaliy.

\subsubsection{The Model and Analysis}

Figure 32 illustrates the model that was employed in the analysis of the subject data. The figure shows that the iiquid film was subdivided into a number of incremental elements of length $\delta x$. The pertinent notation that applies to the general $\mathrm{kth}$ film element is as follows:
(a) $x_{k-1}=(k-1) \delta x=$ the distance from the point of liquid injection to the beginning of the $k$ th film element, $k=1,2,3, \ldots n$.
(b) $u_{k}, p_{k}, \ldots$
- the value for the indicated flow parameter for the kth film element evisiuated at $x_{k}-z_{\bar{x}}, k=1,2,3, \ldots n$.
(c) $x_{e, k}, x_{r, k}$
$=$ the value for the indicated dimen- sional group for the kth film ele- ment evaluated at $x_{k}=\frac{1}{2} \delta x$, $k=1,2,3, \ldots n$. 


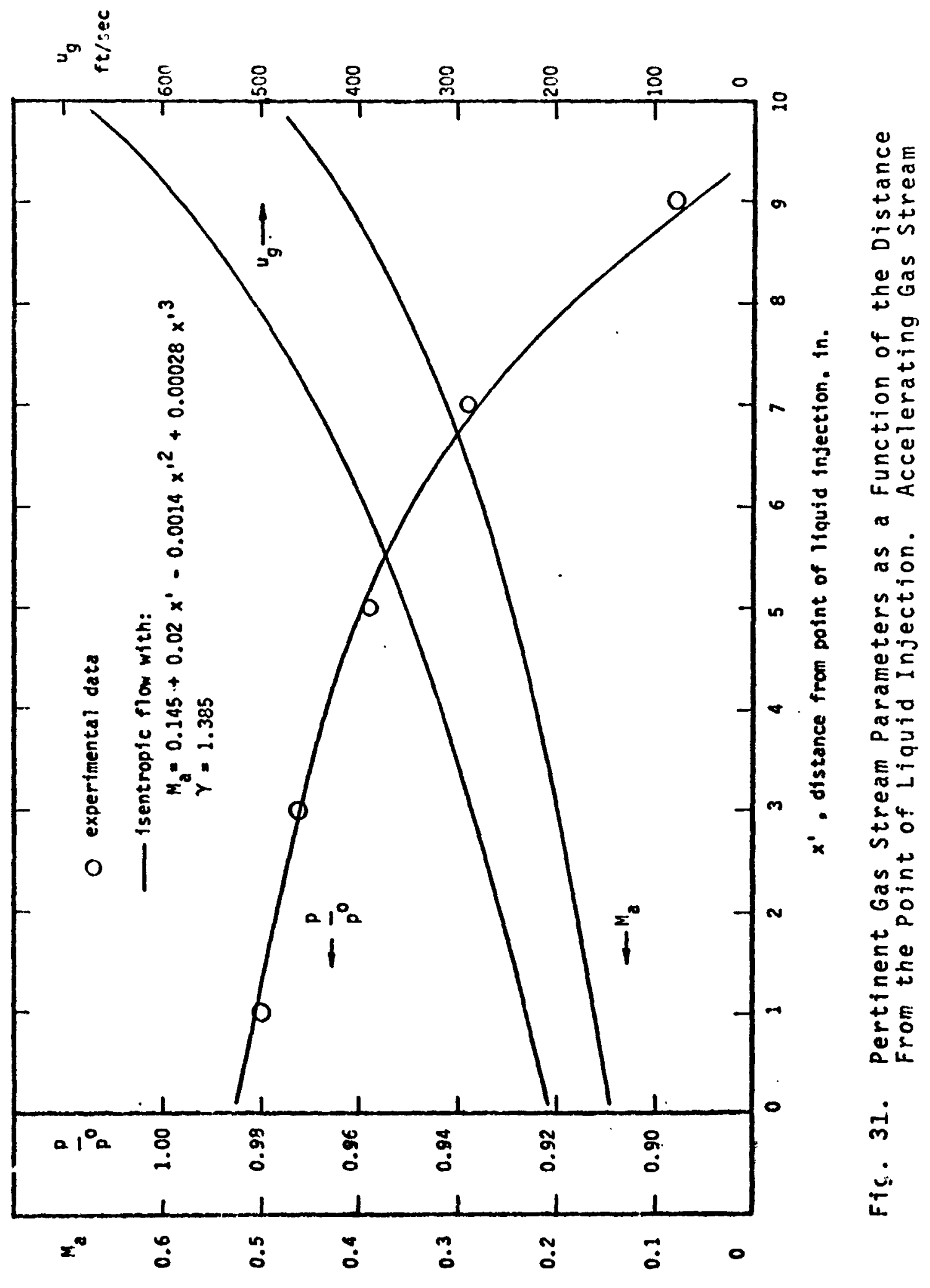

I

]

1

1

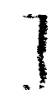

1
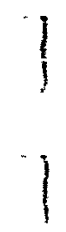

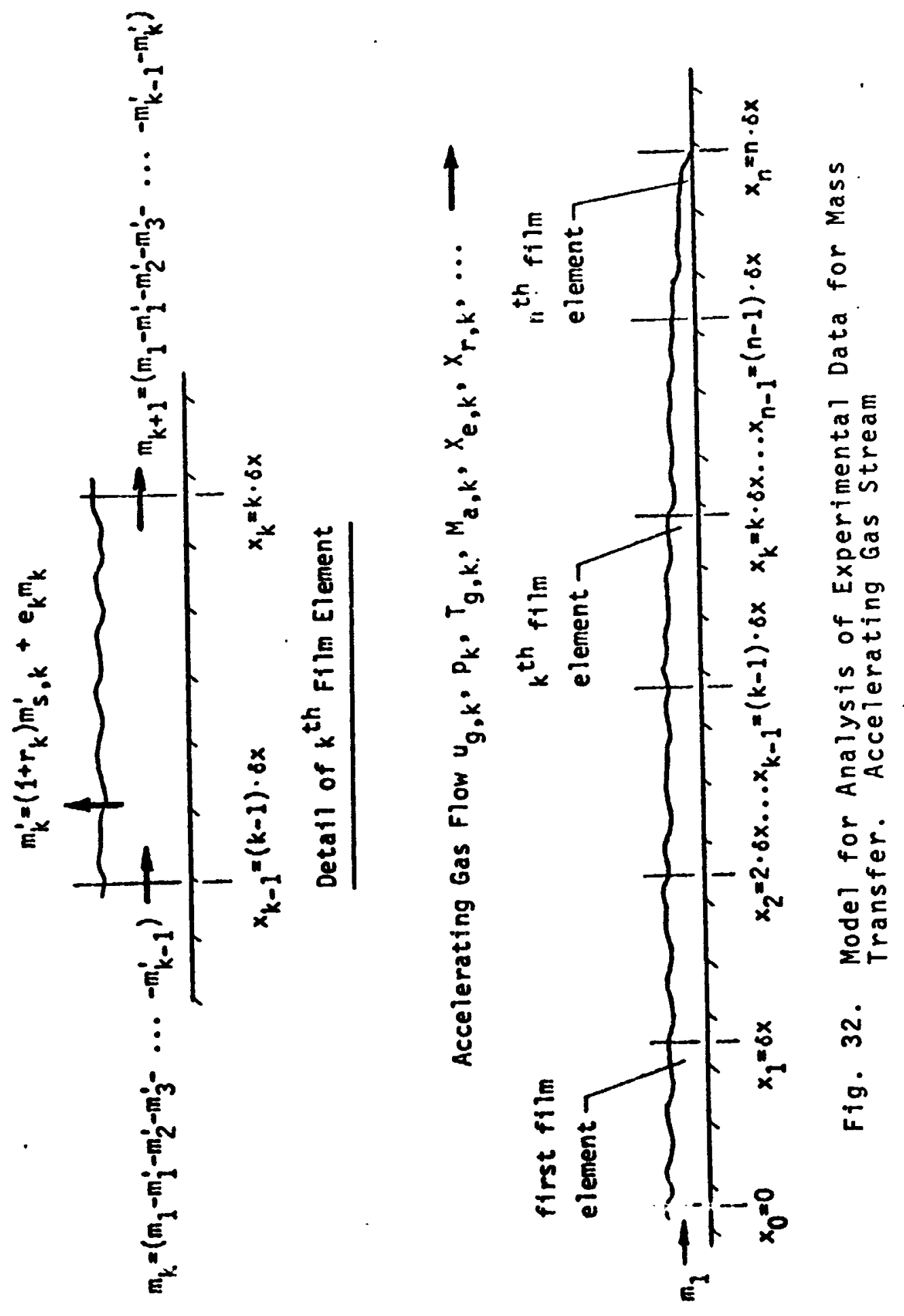
(d) $m_{k}$

(e) $m_{k}^{\prime}$

(f) $m_{s, k}^{i}$
$=$ the rate of liquid flow into the kth film element, $k=1,2,3, \ldots n$.

$=$ the net rate of mass transfer from the $k$ th $f i l m$ element, $k=1,2,3, \ldots n$.

$=$ the rate of simple mass transfer from the kth film element, $k=1,2,3, \ldots n$.

The primary assumption that was employed in the analysis is that the net rate of mass transfer from the kth film element, $m \cdot{ }_{k}^{1}$, is given by the expression

$$
m_{k}^{\prime}=\left(1+r_{k}\right) m_{s, k}^{\prime}+e_{k} m_{k}
$$

where

$$
r_{k}=\frac{3.0}{x_{r, k}^{0.8}}
$$

and

$$
e_{k}=1.0-\exp \left[-5 \times 10^{-5}\left(x_{e, k}-1000\right)\left(\frac{\delta x}{T_{0}}\right)^{d}\right]
$$

The value for the parameter $d$ in Eq. (3-74) was determined from the experimental data.

The procedure that vias employed to calculate the net rate of mass transfer from the liyuid film to the accelerating gas stream for each datum point can be outlined as

* Note that Eq. (3-72) is analogous to Eq. (3-6).

**Note that Eq. $(3-74)$ is motivated by the developments of Subsection 3.2.2. 
follows.

(a) The pertinent gas stream flow parameters were calculated for each location $x_{k}$ following the procedure outlined in Subsection 3.3 .1 .

(b) The entrainment group, $x_{e, k}$, and the roughness group, $x_{r, k}$, were calculated for each location $x_{k}$ from the flow parameters that were determined in step (a).

(c) The rate of simple mass transfer, $m_{s, k}$, for each film element was calculated from the equation

$$
m_{s, k}^{\prime}=m_{s, k}^{\prime \prime} \delta x
$$

where $m_{s, k}^{\prime \prime}$ was computed at the location $x_{k}-\frac{1}{2} \delta x$ from the procedure that was outlined in Subsection 3.3.1.

(d) A value for the parameter d in Eq. (3-74) was selected.

(e) The net rate of mass transfer for the first film element (i.e., $k=1$ ) was calculated from

$$
m_{1}^{\prime}=\left(1+r_{1}\right) m_{s, 1}^{\prime}+e_{1} m_{1}
$$

where $m_{1}$ was the experimentally measured rate of liquid injection.

(f) The net rate of mass transfer from the second film element (i.e., $k=2$ ) was calculated from.

$$
m_{2}^{\prime}=\left(1+r_{2}\right) m_{s, 2}^{\prime}+\varepsilon_{2} m_{2}
$$


where

$$
m_{2}=m_{1}-m_{1}^{\prime}
$$

(g) Steps (e) and (f) were continued in a stepwise fashion so that for the general kth film element the net rate of mass transfer was calculated from

$$
m_{k}^{\prime}=\left(1+r_{k}\right) m_{s, k}^{\prime}+e_{k} m_{k}
$$

where

$$
m_{k}=m_{1}-m_{1}^{\prime}-m_{2}^{\prime}-\ldots-m_{k-1}^{\prime}
$$

(h) When the net rate of mass transfer for each film element had been calculated, the net rate of mass transfer from the entire liquid film was calculated from

$$
m^{\prime} \quad=\sum_{k=1}^{n} m_{k}^{\prime}
$$

where

$$
n=\frac{10}{\delta x}
$$

(i) The result of step (h) was compared with the experimental result for $m^{\prime}\left(=a_{2} ;\right.$. If those two results 
were not in reasonable agreement, then a new value for the parameter d was selected and steps (e) through (h) were repeated until a satisfactory agreement of those results was obtained.

The aforementioned procedure was employed to analyze each of the 50 datum points that was shown in Fig. 17 of Section 2.6. The computations were performed for values of $\delta x$ of $0.1,0.2$, and 0.4 inches to see if that parameter significantly influenced the results. The calculations showed that the simple value of 1.0 for the parameter $p$ in Eq. (3-74) resulted in a good correlation of all of the datum points for each of the aforementioned values for the parameter $\delta x$.

\subsubsection{The Comparison of the Predicted and the} Measured Rates of Mass Transfer

Figure 33 presents the comparison of the experimental data for the net rate of mass transfer from the liquid film to the accelerating gas stream with the results that were predicted from both (a) the simple theory for mass transfer that was outlined in Subsection 3.3.1, and (b) the present theory for mass transfer that was developed in subsection 3.3.2. The stagnation pressure, $p^{0}$, and the film coolant that is represented by each set of experimental data is noted in the figure.

Figure $33(a)$ again graphically fllustrates that the simple theory for mass transfer is, in general, a very poor 


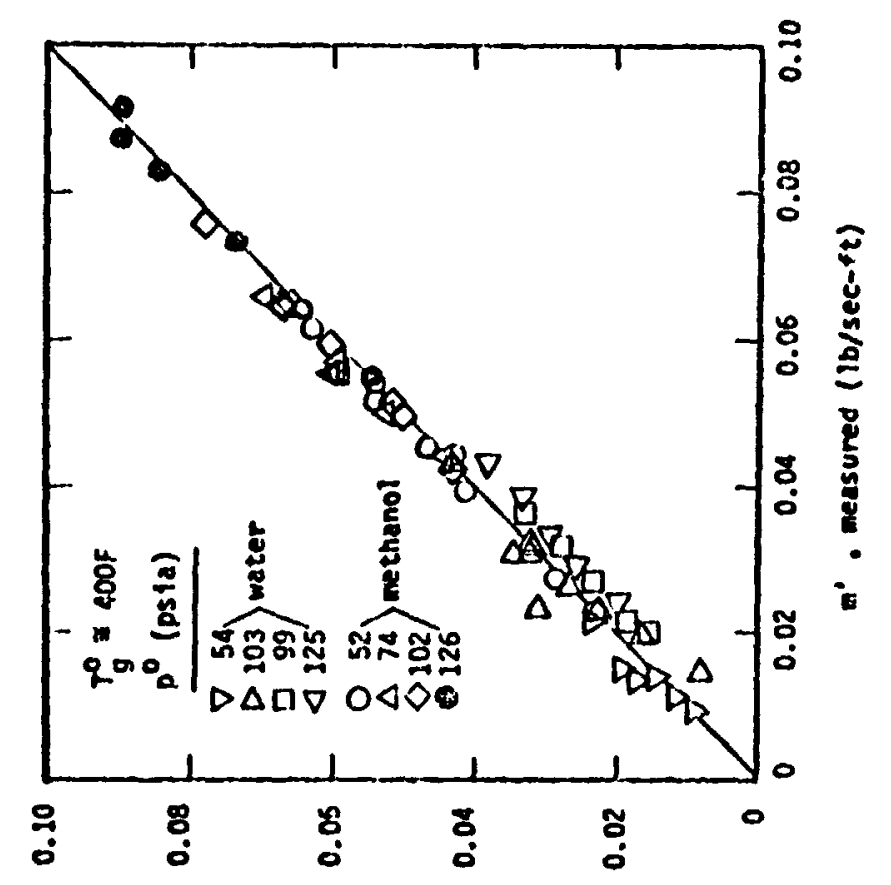

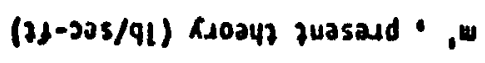

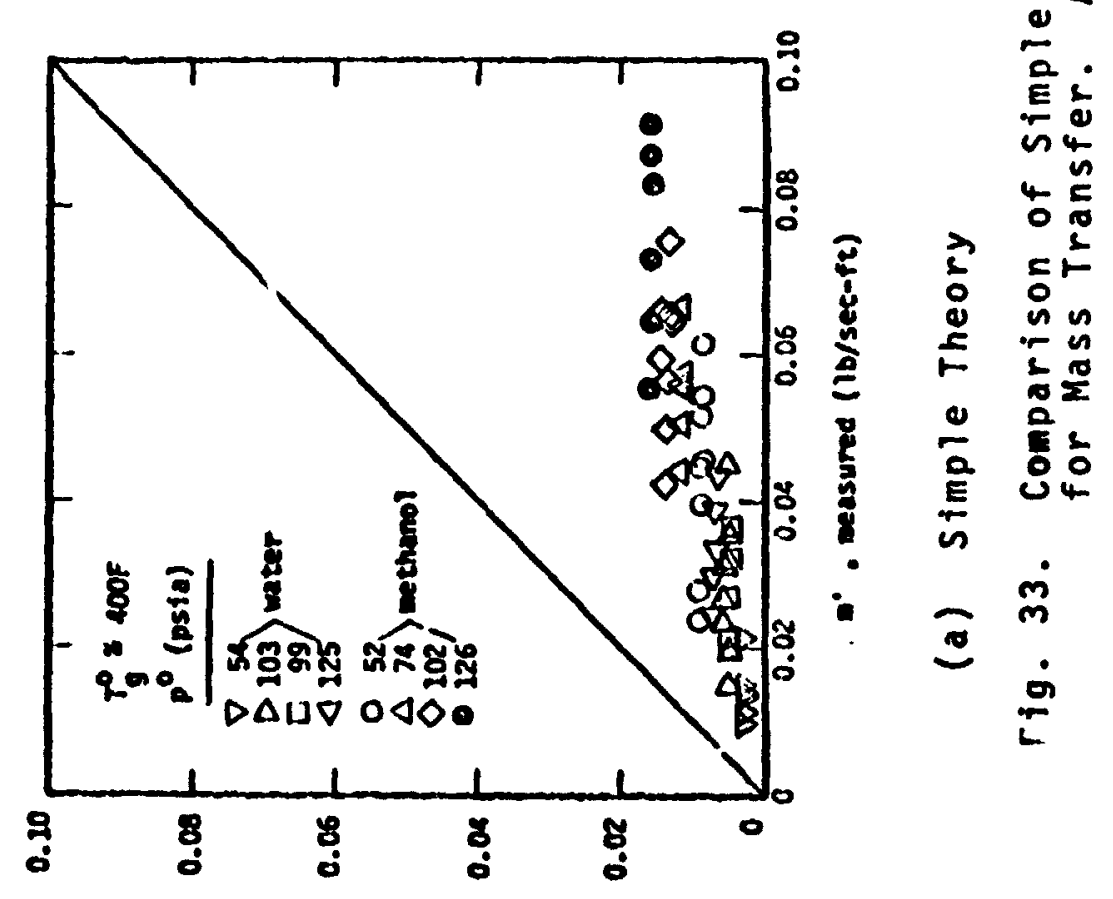

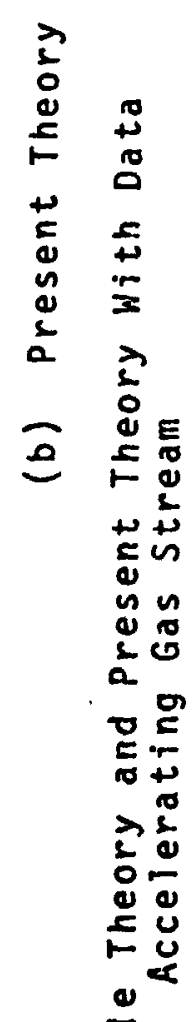

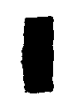

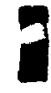

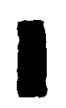

I

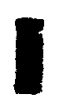

I

I

I

1

1

(21-305/91) Rs004z o1du1s + ?4. 
approximation to the complex phenomena that characterize liquid-film cooling. The maximum difference between the measured and the analytical result that is shown in the figure occurs for methanol as the liquid phase at a measured rate of mass transfer of $0.1668 \mathrm{lb} / \mathrm{sec}-\mathrm{ft}$ and a corresponding predicted value of $0.0309 \mathrm{lb} / \mathrm{sec}-\mathrm{ft}$, a difference of more than 500 percent.

Figure $33(b)$ shows that the present theory, with a value of 1.0 for the parameter $d$ in Eq. (3.74), resulted in a satisfactory correlation of the experimental data. The maximum difference between the measured and the predicted result occurred for water as the film coolant at an experimental value for the net mass transfer rate of 0.0462 $1 \mathrm{~b} / \mathrm{sec}-\mathrm{ft}$ and a corresponding predicted value of $0.06 \hat{c} 0$ $1 \mathrm{~b} / \mathrm{sec}-\mathrm{ft}$, a difference of less than 40 percent.

The utility of the present analysis is investigated in further detail in Section 4 . Considered in that section is the correlation of the data that were reported in the primary reference (24) for the liquid-film cooling of a Mach number 1.92, axi-symmetric nozzle. Also presented in that section is an illustrative example of the coolant requirements that are predicted by the present theory to $c 001$ an exhaust nozzle for which the gas stream has a stagnation temperature of $4000 R$ and a stagnation pressure of 500 psia. These stagnation conditions were chosen as being typical of those that characterize the gas flow in the exhaust nozzle 
of a high-energy propulsion device.

\subsection{Correlation of the Experiment.al Data for the Maximum Liquid Temperature}

The correlation of the experimental data that were reported in section 2.7 for the maximum liquid temperature, $T_{1, m}$, is considered in this section. Subsection 3.4 .1 presents a simple theory for calculating that liquid temperature. The comparison of the theoretical and the experimental results is shown in subsection 3.4.2.

\subsubsection{A Method for Predicting the Maximum Liquid Film Temperature*}

The maximum liquid-film temperature, $T_{1}, m$, can be related to the bulk flow parameters for the gas stream by specifying (a) the appropriate energy balance across the gas-liquid interface, and (b) an analogy between the rates of convective*t heat and mass transfer. The discussion of Section 2.7 showed that the maximum liquid temperature is obtained when all of the energy transfer to the liquid film results in vaporization of liquid in the film, with none of the energy transfer resulting in an increase in the sensible enthalpy for the liquid in the film. It follows from the discussion of the interfacial energy balance in subsection 3.1.2 that the maximum liquid temperature is obtained when

* This method is essentially the same as that previously presented by the author in Refs. (25) and (26).

* Mass transfer due to entrainment is not considered. 
the energy balance parameter $\phi$ at the surface of the liquid film reduces to

$$
\phi \quad=H_{v, m}
$$

where $H_{v, m}$ is the heat of vaporization for the liquid evaluated at the maximum liquid temperature $T_{1, m}$.

The simplest analogy between convective heat and mass transfer is that given by (36)

$$
s t_{s}=s t_{s}^{\prime}
$$

where St' is the stanton number for simple mass transfer defined by

$$
s t_{s}^{\prime}=\frac{m_{s}^{\prime \prime}}{G} \frac{p-P_{v, m}}{P_{v, m}} \frac{M_{g}}{M_{v}}
$$

The term $P_{f, m}$ in Eq. (3-85) is the partial pressure for the vapor at the surface of the liquid film evaluated at the temperature $T_{1, m}$.

It is important to note that this analogy, although very simple, should be approximately correct for quite general flow conditions, including the case of accelerating

*Equation (3-85) can also be written in terms of the concentration of the vapor at the surface of the liquid film, $C_{s}$. Thus

$$
s t_{s}^{\prime}=\frac{m_{s}^{n}}{6} \frac{1-C_{s}}{c_{s}}
$$


gas flow and the case where considerabl! entrainment of liquid into the gis stream is occurring. In other words, the acceleration of the gas stream and/or the entrainment of liquid should have a similar influence on the development of both the thermal and the vapor-concentration boundary layers over the surface of the liquid film.

Combining Eq. (3-15) with Eqs. (3.83) - (3-85) yields

$$
\begin{aligned}
\frac{p-P_{v, s}}{F_{v, s}} \frac{M_{g}}{M_{v}} & =\frac{\phi}{c_{p g}\left(T_{g}-T_{s}\right)} \\
& =\frac{H_{v, m}}{c_{p g}\left(T_{g}-T_{1, m}\right)}
\end{aligned}
$$

Equation (3-87b) is the desired analytical expression. Since a value for tre liquid temperature $T_{1, m}$ uniquely determines the value for both the vapor pressure $p_{v, m}$ and the heat of vaporization $H_{v, m}$, it follows from Eq. (3-87b) that the meximum liquid temperature is expressed solely in

*Note that if Eqs. (3-85) and (3-86) are combined to eliminate the left-hand side of Eq. (3-87a), and if the parameter $B_{h}$ is introduced, then the resultant expression can be written in the form

$$
c_{s}=\frac{B_{h}}{1+B_{h}}
$$

Equation (3-26) was introduced in Subsection 3.1 .2 without derivation. 
terms of the flow parameters $p, T_{g}, c_{p g}$, and $M_{g}$ for the gas stream.

Figure 34 presents, for purposes of illustration, calculated values of the maximum liquid temperature $T_{1, m}$ for an air-water system*. Shown in the figure is a plot of $T_{1, m}$ as a function of the static pressure $p$ with the gas stream temperature $T_{g}$ as a parameter. The saturation curve for water that is presented in the figure illustrates the approximate error that is introduced by the assumption that the maximum film temperature is equal to the saturation temperature for the liquid coolant at the local static pressure, p. That assumption was employed, for example, by Refs. $(20,30,23,42)$. References $(25),(26)$ and (28) suggest that the calculated results shown in Fig. 34 are in good agreement with the reported experimental data for the film temperature $T_{1, m}$ for an air-water system.

\subsubsection{Comparison of the Experimental and the Predicted Results for the Maximum Film Temperature}

Section 2.7 previously discussed the nature of the experimental measurements for the maximum liquid temperature $T_{1, m}{ }^{* *}$. Presented in Fig. 18 of that section was a comparison

* The calculations were performed by employing Eq. (3-87b) in conjunction with the tabulated data of Refs. (40) and (41). A sample calculation is presented in Appendix F.

**Recall from Section 2.7 that the maximum 1 iquid temperature $T, 1, m$ was determined experimentally by measuring the temperature for the liquid at the point of liquid withdrawal, $T_{1,2^{*}}$ 


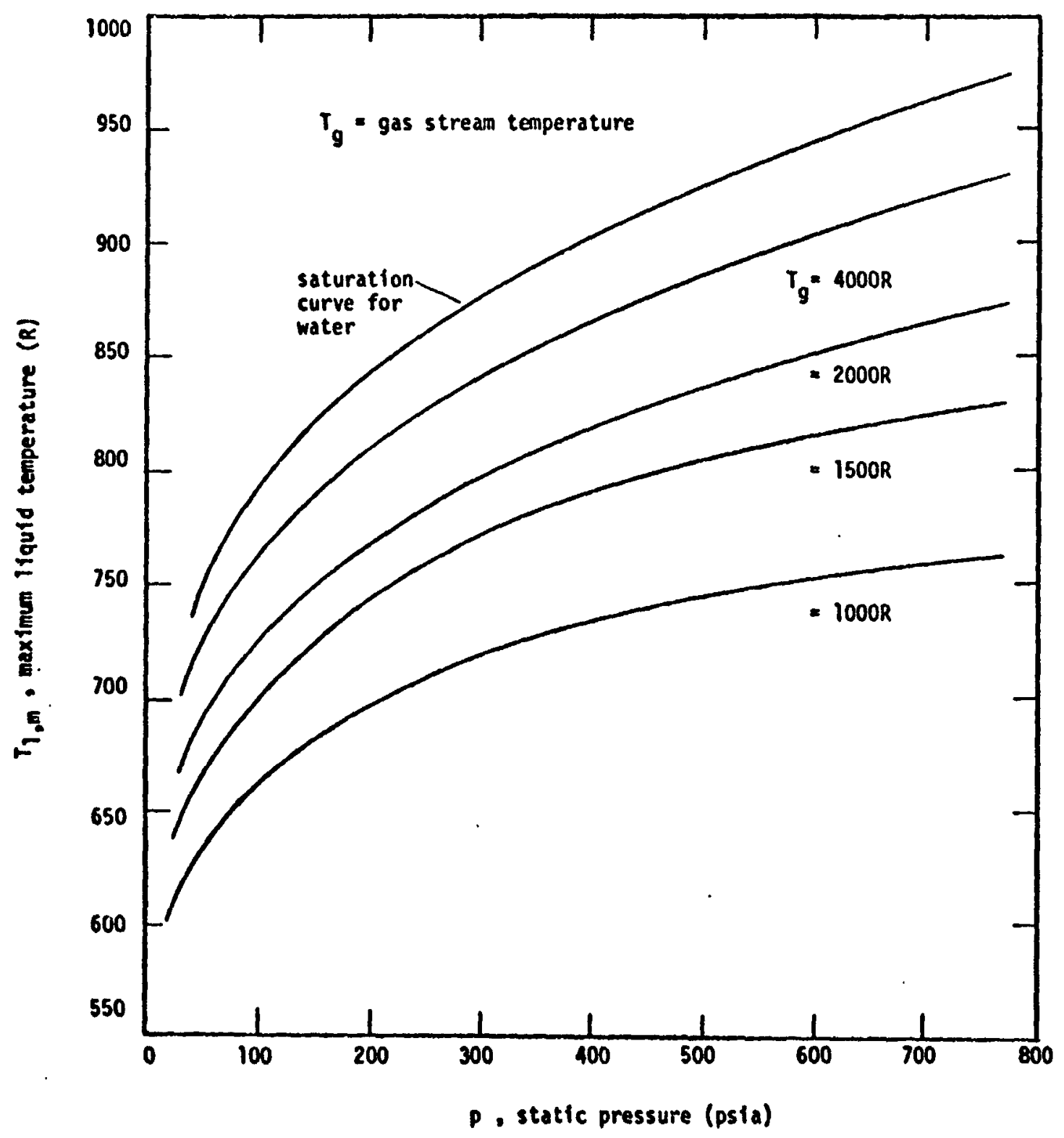

Fig. 34. Computed Results for Maximum Liquid Temperature as a Function of $T_{g}$ and $p$ for an Air-Water System 
between the temperature $T_{1, m}$ and the boiling temperature for the various liquids* of the subject investigation at the prevailing gas stream pressure. Figure 18 illustrated that the difference between those two temperatures was generally significant, with the maximum difference for the subject investigation being equal to approximately $350 \mathrm{~F}$. A similar comparison is made in this section between the experimental values for the maximum liquid temperature and those that are predicted by the theory outlined in the foregoing subsection.

The primary equation (3-87b) was employed, together with the thermodynamic data presented in Appendix $D$ of the present report, to calculate the values for $T_{1}, m$ for the gas stream pressure and temperature corresponding to the experimental tests conducted. Figure 35 illustrates the results of those computations. Presented in the figure is a plot of the measured value for the liquid temperature versus the predicted value for the temperature $T_{1, m}$. The cross-hatched areas in Fig. 35 represent the range of the experimental values for the maximum liquid temperature that were realized for each liquid at a particular value for the gas stream temperature and pressure**. The agreement between the experimental and : ralytical results shown in Fig. 35 is generally quite satisfactor.". The only substantial

* water, methanol, butanol, and RP-1

**Several hundred datum points are represented by the cross-hatched areas in Fig. 35 . 


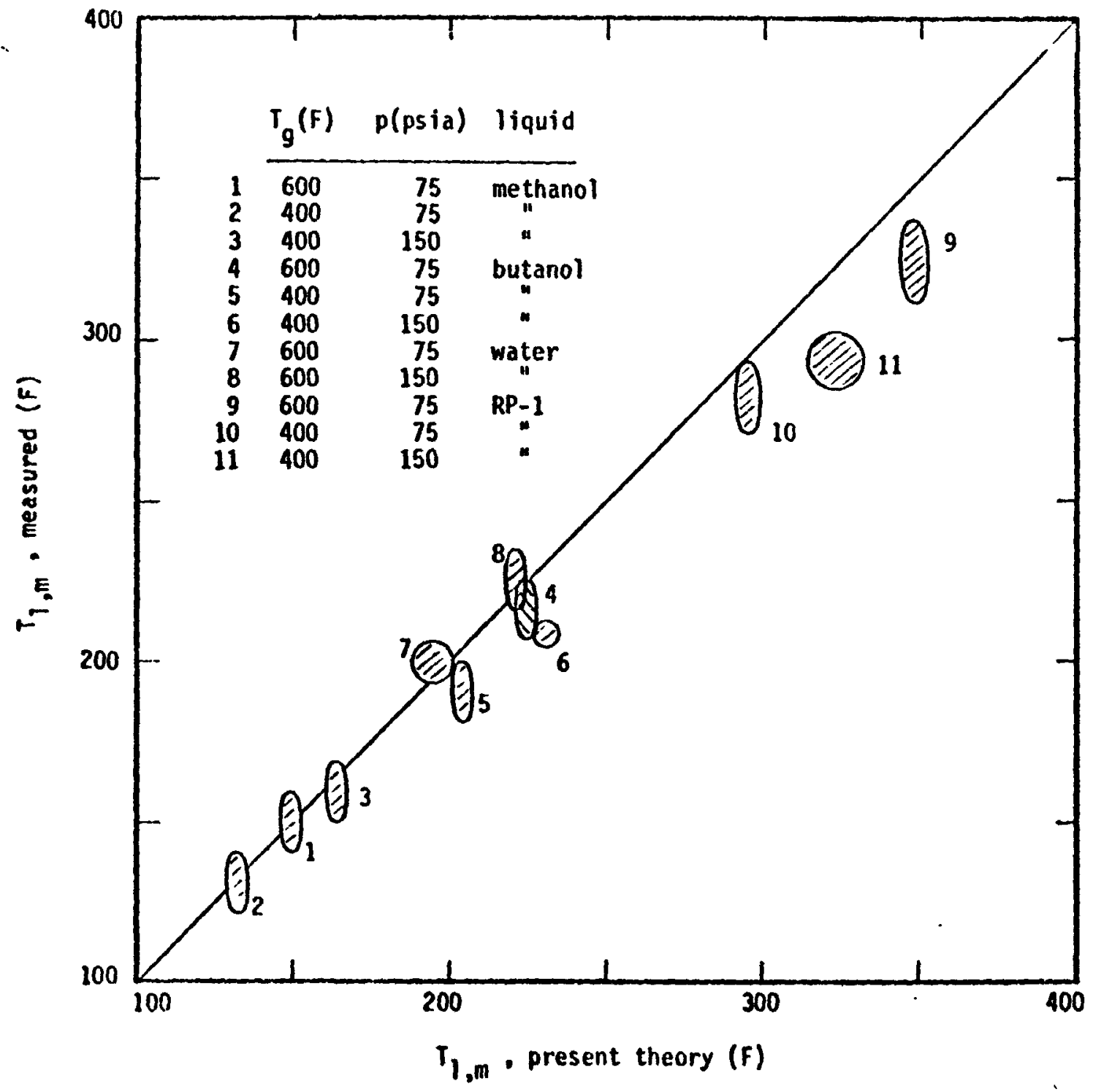

Fig. 35. Comparison of Present Theory With Data for Maximum Liquid Temperature 
difference between the measured and the predicted values for $T_{1, m}$ occurs for some of the experimental data that were obtained with RP-I as the film coolant. However, part of that difference may be due to the fact that RP-I was the liquid for which the maximum temperature rise occurred after it was introduced onto the test plate. Therefore, the liquid film may not have always achieved its maximum temperature by the time the film reached the point of withdrawal. However, even if that were not the case, the results of Fig. 35 suggest that the simple theory presented herein for the prediction of the maximum film temperature is sufficiently accurate for the purposes of most engineering-type calculations. 


\section{CORRELATION OF THE DATA OF THE PRIMARY REFERENCES}

\subsection{Introduction}

The experimental data for mass transfer that are reported in the subject report are limited in two fundamental respects: (a) the liquid-film cooled length was held constant at 10 inches, and (b) the gas stream temperature was limited to o00F and the gas stream pressure was limited to 150 psia so that the experimental problems would be minimized. The purpose of the present section is to analyze those data that have been reported in the literature for the problem of liquid-film cooling that are not so restricted. The analysis of those data will give some insight into the general usefulness of the correlation for mass transfer that is presented in the subject report.

The experimental data that are reported by the primary references (19) (20) and (24) are analyzed. The data reported by Kinney, et al. (19) and those reported by Emmons (20) were obtained under essentially constant gas sijedm velocity conditions. The basic experimental configuration for each of those two investigations was the flow of a hot gas through a horizontally mounted tube with the liquid film introduced around the circumference of the tube. 
The experimental data reported by Hermann, et al. (24) are for the case of a strongly accelerating gas flow. They investigated the problem of liquid-film cooling an axisymmetric convergent-divergent nozzle. For that investigation, the gas stream was accelerated from a Mach number of approximately 0.07 at the plane of liquid injection to a Mach number of 1.92 at the exit of the nozzle.

Table 3 presents a comparison of the nominal flow parameters for the experimental investigation of Kinney, et al. and of Emmons with those for that portion of the present investigation where the gas stream velocity was essentially constant over the length of the wetted test plate. Table 3 shows that the experimental parameters for the subject references represent a substantial extension over those for the experimental program reported herein. For example, the maximum gas stream temperature, $T_{g}$, investigated in both references is significantly greater than the maximum temperature of $600 \mathrm{~F}$ for the present investigation. Moreover, the gas stream pressure that was employed by Emmons is more than three times that employed in the subject investigation; the maximum gas stream velocity that was investigated by Kinney is nearly four times the maximum that is reported herein; and the maximum film cooled length that is reported by Kinney is more than three times longer than the 10-inch film cooled length that was employed in the present invest1gation. 


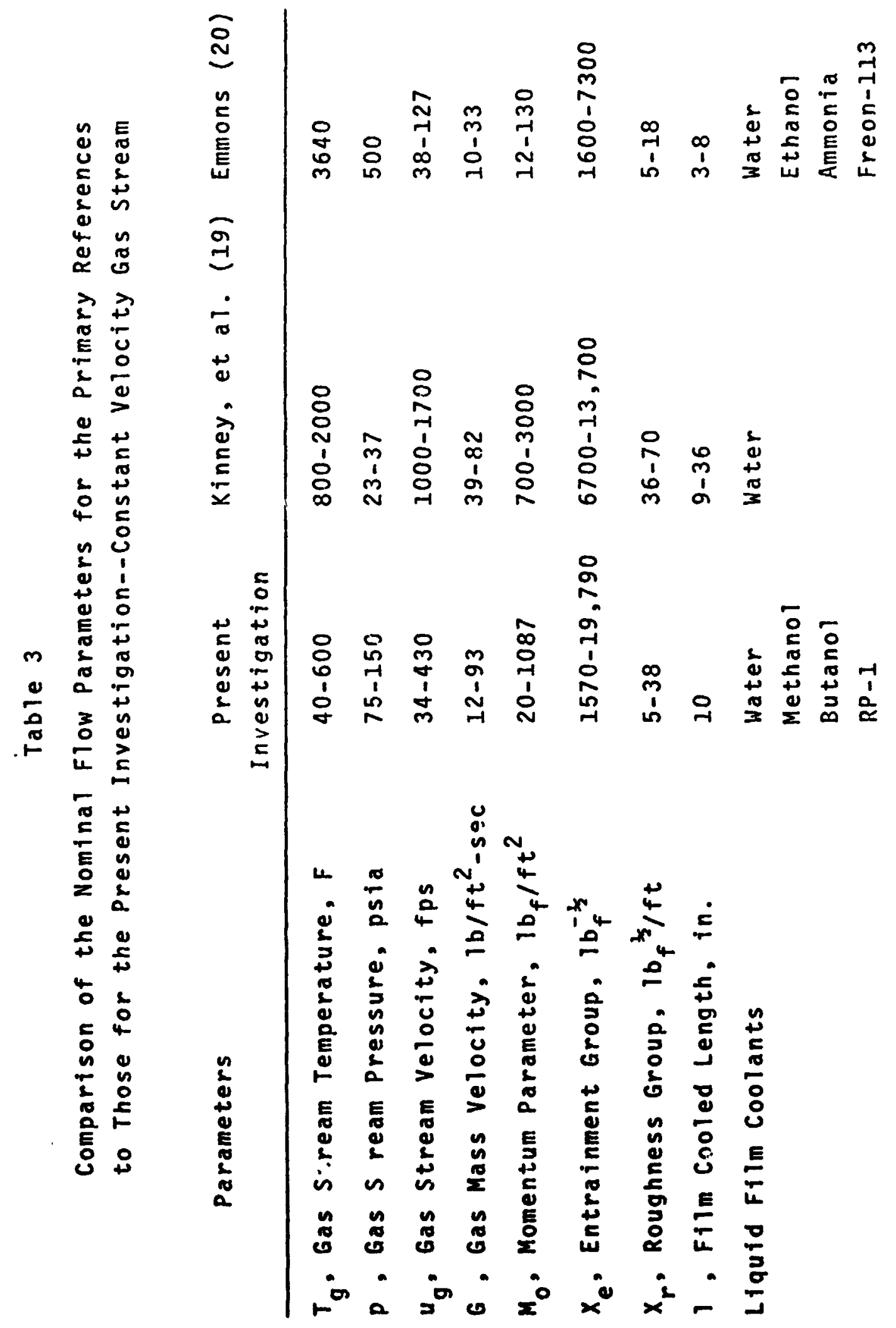


Table 4 presents a similar comparison of the nominal flow parameters for the experimental investigation of Hermann, et al. With those for that portion of the subject investigation where the gas stream was accelerated over the length of the film cooled test section. Again, the experimental parameters for the referenced investigation represent a considerable extension over those for the experimental program reported herein, particularly with reference to the Mach number, $M_{a}$, the gas stream velocity, $u_{g}$, and the staticstagnation pressure ratio, $\mathrm{p} / \mathrm{p}^{0}$.

Obviously, any evaluation of the present theory in terms of the referenced experimental data is only as conclusive as is the accuracy of those experimental data. In that regard, it should be mentioned that the wetted surface area (and therefore the net rate of mass transfer from the liquid film to the gas stream) was determined in each of the subject references by external wall temperature measurements. Moreover, each reference employed the assumption that the liquid film terminated at that point where the wall temperature just exceeded the boiling temperature for the liquid at the prevafling local static pressure. Section 3.4 herein showed, however, that that assumption is never exactly correct, with the error in the assumption increasing with decreasing gas stream temperature and/or decreasing gas stream pressure. Thus, there is some limitation in accuracy introduced by the experimental technique employed 
Table 4

Comparison of the Nominal Flow Parameters of the Primary Reference (24) to Those of the Present Investigation--Accelerating Gas Stream

Parameter

Present

Reference (24)

Investigation

$T_{g}^{0}$, Gas Stream Stagnation
Temperature, $R$

860

$1460-1940$

$p^{0}$, Gas Stream Stagnation

Pressure, psia

$50-150$

69-82

$p / p^{0}$, Static-Stagnation

$0.84-0.98$

$0.14-0.99$

Pressure Ratio

$0.95-0.99$

$0.58-0.99$

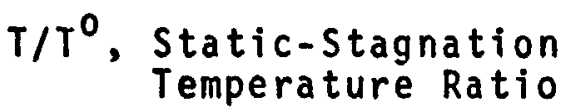

$M_{a}$, Gas Stream Mach Number

$0.15-0.51$

$0.07-1.92$

$u_{g}$, Gas Stream Vrincity, fps

$200-660$

$150-2750$

Liquid Film Coolants

Water

Water

Methanol

$+$

I

I

I

1

1

1

)

1

) 
in the aforementioned investigations.

Furthermore, Kinney, et al. (19) comment that the experimental data for mass transfer that they reported could be reproduced in only a few cases to within 5 percent, and more generally to within only 10 or 15 percent. They also state that the accuracy of the experimental data for the short film cooled lengths--less than, say, 12 inches--was poorer than that for the longer film cooled lengths that were investigated. The problem that they experienced for the shorter wetted lengths was that the liquid film seldom terminated uniformly around the circumference of the wetted tube. Thus, it was difficult to interpret the external wall temperature measurements and to accurately determine the actual wetted surface area. Of the three references considered in the present section, the research program reported by Kinney, et al. is considered the most comprehensive and was conducted under the most well controlled experimental conditions. Thus the confidence in the accuracy of the experimental data for Refs. (20) and (24) is somewhat less than that for the experimental data of Ref. (19) because of the short film cooled lengths that were employed in those two investigations.

One additional problem occurred in the evaluation of the theory of the present report from the data that were presented in the subject references. Namely, how to properly determine the value for the surface tension o that 
enters into the entrainment group $x_{e}$. In each of the three subject references, the liquid coolant was injected at essentially the ambient temperatura, so that a substantial change occurred in the value for the temperature of the liquid (and therefore the value for $\sigma$ ) over tha length of the wetted test section. This problem was particularly acute for the investigation due to Emmons because the final equilibrium film temperature at the end of the liquid:. Im was generally very high. For exainple, for the film cooling experiments reported by Emmons with water as the liquid phase, the temperature for the water increased from approximately $70 \mathrm{~F}$ at the point of liquid injection to someihing in excess of $400 \mathrm{~F}$ at the terminus of the liquid film. The corresponding decrease in the surface tension for the water coolant was roughly 50 percent. Obviously, the change in the temperature of the liquid coolant over the length of the wetted test section creates some uncertainty about how tre parameter $x_{e}$ shouid be evaluated. It is also obvious, however, that such a variation in the liquid film temperature would characterize the general application of liquidfilm cooling. Because of the genera importance of the problem of determining a proper reference temperature for a... calculation of the surface tension $\sigma$, this Frobien is discussed in greater detail in section 4.2 that follows. Sections $4.3,4.4$, and 4.5 consider the analysis of the data reported in Refs. (19), (20), and (24), respectively. 
The basic assumptions that were employed to analyze those experimental data are listed in each section, and a comparison is made in each case between the reported rates of mass transfer and those that are predicted from the present theory.

\subsection{An Approximate Method for Evaluating the Surface Tension}

An approximate mathod for the evaluation of a reference liquid temperature, which will be denoted by $T_{1, r}$, that can be employed to estimate the value for the sirface tension in the group $x_{e}$ is presented in this section. The method strictly applies to only the case where the wetted surface is adiabatic*, but it could be readily extended to the more general case where the film cooled region is also cooled externally.

For the general case of liquid-film cooling, the bulk temperature for the liquid increases from a minimum value at the point of injection, $T_{1,1}$, to a maximum value at (or near) the end of the liquid film. That maximum film temperature, denoted by $T_{1, m}$, can be estimated from the theory outlined previously in Subsection 3.4.1. The basic problem, therefore, is to determine how the minimum and the maximum film temperatures should be "weighted" so as to determine the reference temperature $T_{1, r}$.

First it is convenient to introduce a new parameter $\bar{\phi}$

*This case includes the subject references $(19),(20),(24)$. 
which is defined as the average value for the energy balance parameter $\phi$ over the length of the liquid film. The parameter $\bar{\phi}$ can be reasonably approximated by the following expression. Thus*

$$
\bar{\phi}=H_{v, m}+h_{1, m}-h_{1,1}
$$

where

$$
\begin{aligned}
h_{1, m}= & \text { the sensible enthalpy for the } 1 \text { iquid evaluated } \\
& \text { at the temperature } T_{1, m}
\end{aligned}
$$

The rate at which the bulk temperature for the liquid film approaches the maximum film temperature, $T_{1, m}$, once that it is injected onto the test plate is a function of how much of the total energy transfer to the surface of the liquid film results in an increase in the sensible enthalpy of the liquid and how much results in the evaporation of liquid. That is, it is dependent on the relative magnitudes of the terms $H_{v, m}$ and $\left(h_{1, m}-h_{1,1}\right)$ on the right of Eq. (4-1). Thus, a weighting parameter, denoted by "w", can be

*It can be shown that in most cases, the group of terms $\left(H_{v}+h_{1}-h_{1,1}\right)$, where $H_{v}$ and $h_{1}$ are evaluated at any temperature between $T_{1,1}$ and $T_{1, m}$ is essentially a constant over that range of liquid temperatures icij. Thus the evaluation of $H_{v}$ and $h_{1}$ at $T_{1, m}$ is mostly a matter of
convenience. 
defined as follows:

$$
w=H_{v, m} / \bar{\phi}
$$

To illustrate: if the value for $w$ is large (i.e., near unity) so that most of the energy transfer to the liquid film results in evaporation of liquid, then the reference temperature $T_{1, r}$ should be chosen as neariy equal to the maximum film temperature $T_{1, m}$. However, if $w$ is very small, so that most of the energy transfer to the liquid film results in an increase in the sensible enthalpy of the liquid, then the average temperature for the liquid over the length of the wetted test surface will be much less than $T_{1, m}$, and the reference temperature $T_{1, r}$ should be weighted more in favor of the minimum liquid temperature $T_{1,1}$. Therefore, in view of the lack of any definitive data for the problem, it is suggested herein that the reference temperature $T_{1, m}$ be approximated by the following expression. Thus

$$
T_{1, r}=w T_{1, m}+(1-w) T_{1,1}
$$

Equation (4-3) was employed to estimate the surface tension for the analysis of the data of the primary references (19) and (20). The values that were computed for $T_{1, r}$ are given in the appropriate subsection. 


\subsection{The Data Due to Kinney, Abramson, and Sloop*}

\subsubsection{Introductory Remarks}

Kinney, et al. (19) present experimental data for the liquid-film cooling of a 2-inch and two 4-inch inside diameter ducts. The 2-inch diameter duct and one of the 4-inch diameter ducts were seamless tubes having a honed inner surface. Tirese two ducts are termed herein the smooth-surface ducts. The second 4-inch diameter duct was a 4-inch inside diameter rolled tube with a longitudinal weld. Although the inside surface of the tube was machined after welding, some surface roughness and waviness remained. This tube is termed herein the rough-surface tube.

Subsection 4.3.2, which follows, describes the basic experimental apparatus for Ref. (19). The primary assumptions that were employed in order to obtain a comparison of the subject data with the theory that is developed in the present report are outlined in Subsection 4.3.3. Subsection 4.3.4 then presents the comparison between the reported experimental data and the calculations from the present theory.

\subsubsection{The Basic Apparatus}

Figure 36 illustrates schematically the basic experimental apparatus employed in the investigation reported in Ref. (19). The basic apparatus consisted of a horizontally *Refer to Table 3 for the nominal flow parameters for these data. 
161

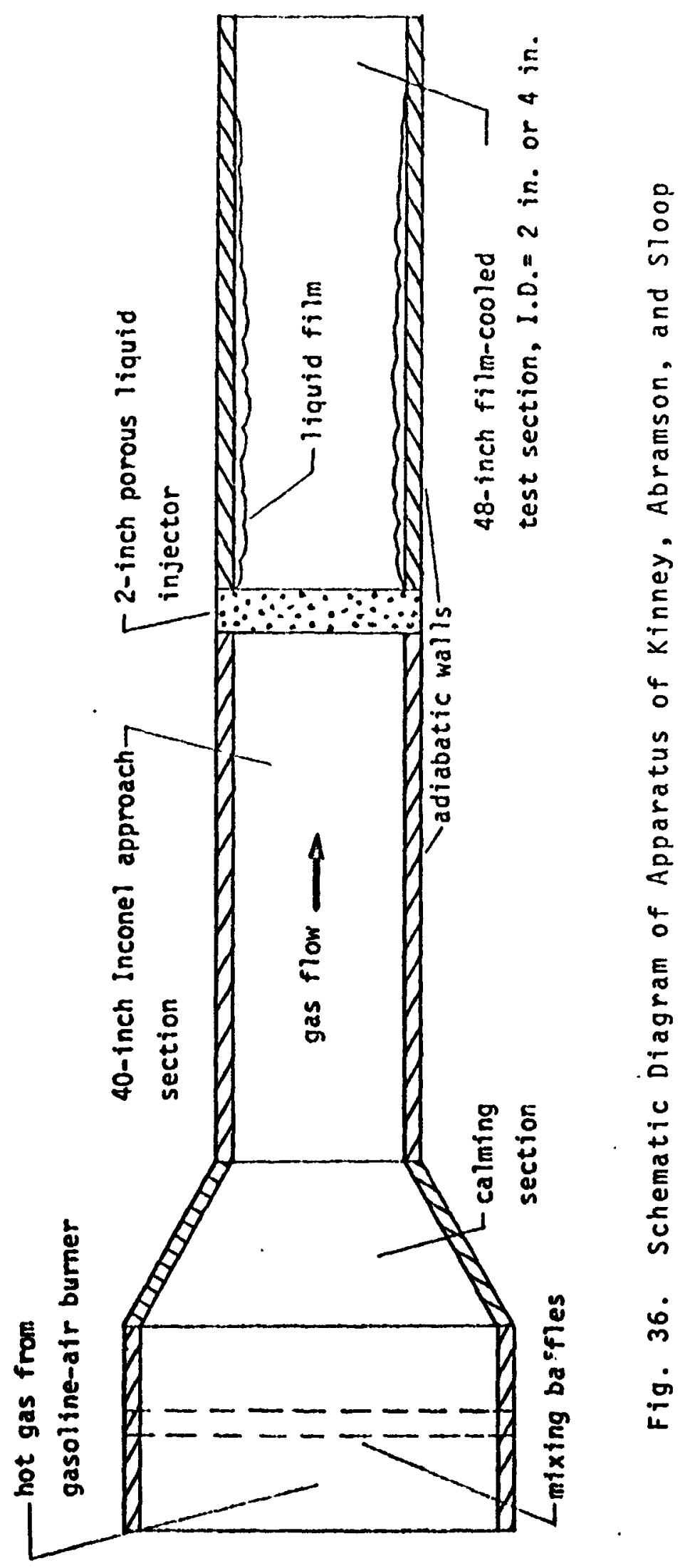


mounted Inconel approach section that was 40 inches 10 ng and a film cooled test section that was 48 inches long. The walls of the experimental duct work were essentially adiabatic. Mixing baffles and a calming section were installed upstream of the approach section in order to obtain a uniform gas stream having a relatively low level of turbulence. A gasoline-air combustor was employed to produce the hot gas stream.

The liquid injector that was employed was fabricated from a porous wire cloth ( 36 mesh) that was mounted flush with the internal flow passage. The porous injector element was two inches long in the direction of the gas flow. The data reported were for a water film.

\subsubsection{The Assumptions}

The basic assumptions that were employed to analyze the experimental data for the subject reference are as follows: (a) The gas flow was treated as boundary-layer type flow over a flat plate. The velocity boundary layer was assumed to be fully turbulent and to develop from the leading edge of the 40-inch long approach section.

(b) The approach section was assumed to be adiabatic so that a thermal entrance length $x_{t}=40$ inches existed. (c) The thermodynamic properties for the gas stream were assumed to be the same as those for dry air at the prevailing gas stream temperature. The value for the gas stream 
Prandt 1 number, $\mathrm{Pr}$, was taken to be 0.7 for all computations.

(d) Radiation heat transfer was assumed negiigible.

(e) The reference temperature $T_{1, r}$, for the evaluation of the surface tension $\sigma$ in the entrainment group $x_{e}$, was estimated from the procedure outlined in Section 4.2 to be $165 \mathrm{~F}$ for the lowest gas stream temperature that was investigated ( $800 \mathrm{~F})$, and $210 \mathrm{~F}$ for the highest gas stream temperature that was investigated $(1600 \mathrm{~F})$. The values for the reference temperature for intermediate values of the gas stream temperature were determintr by a linear interpolation between those limiting values.

(f) The value for the energy balance parameter $\phi$ was calculated from Eq. (4-1).

\subsubsection{Comparison of the Present Theory With the Experimental Data}

The primary equation of the present theory is repeated here for convenience. Thus

$$
\frac{m_{1}}{m_{s}^{T}}=\left(1.0+\frac{3.0}{x_{r}^{0.8}}\right) \exp \left[5.0 \times 10^{-5}\left(x_{e}-1000\right)(1 / 1)^{b}\right]
$$

Equation (3-64) gives the desired relationship between the rate at which liguid is injected, $m_{1}$, and the resultant film cooled length, 1 . The simple rate of mass transfer, $m_{s}^{\prime}$, was computed from the procedure outlined in subsection 3.1.2, together with the aforementioned assumptions. Figure 29 of 
Subsection 3.2 .2 predicts a value of approximately 0.6 for the parameter $b$ in Eq. (3-64) for the subject flow situation. The present subsection will investigate the validity of that prediction (and, therefore, the validity of the model that was employed in the analys is of subsection 3.2.1).

Figure 37 presents a comparison of the experimental data for mass transfer in terms of the film cooled length versus the injected liquid flowrate for the 4-inch diameter, smooth-surface tube with the results that were computed for both (a) the simple theory for mass transfer (Fig. 37 (a)), and (b) the present theory for mass transfer (Fig. 37(b)). Figure 38 presents a similar comparison for the 2-inch diameter, smooth-surface tube. The solid curves presented in these two figures represent the data that are presented in Fig. 6 of Ref. (19). Approximately 25 datum points are represented by each of the solid curves. The values for the gas stream temperature and the gas stream Reynolds number (based on the tube diameter $D$ ) are indicated in the figures for each set of experimental data.

Figures 37 (a) and $38(a)$ again illustrate how inadequate the simple theory is for the problem of liquid-film cooling. For example: Figure $37(a)$ shows that at a gas stream Reynolds number of $4.7 \times 10^{5}$ and a gas stream temnerature of $1600 F$, the value for $m_{1}$ required to establish a film cooled length of $2.2 \mathrm{ft}$ is more than three times the value for $\mathrm{m}_{1}$ that is predicted from the simple theory for mass transfer. 


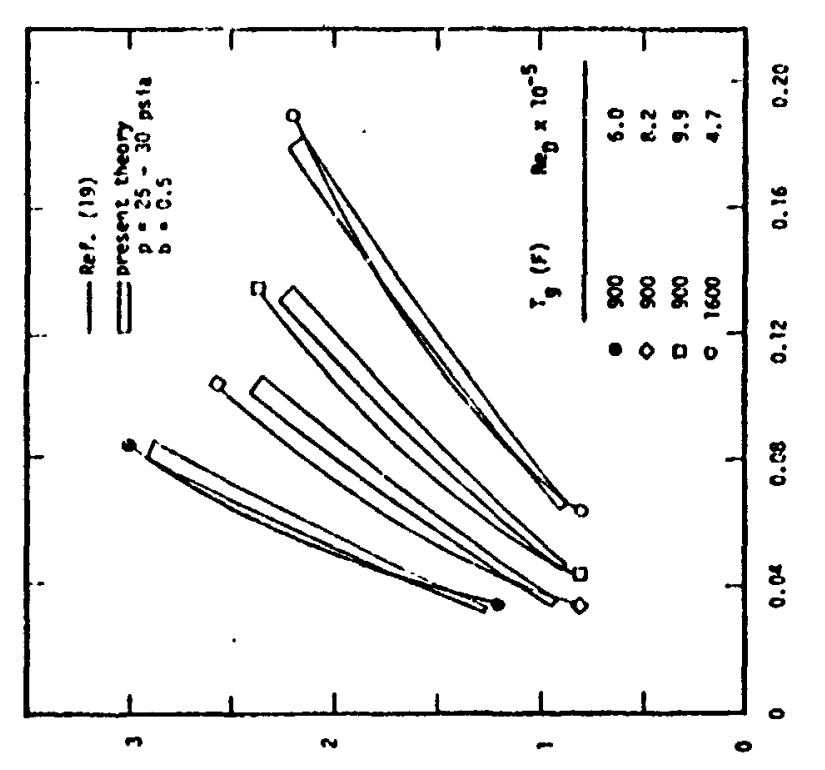

(ii) 4rbual paposs-wi1)

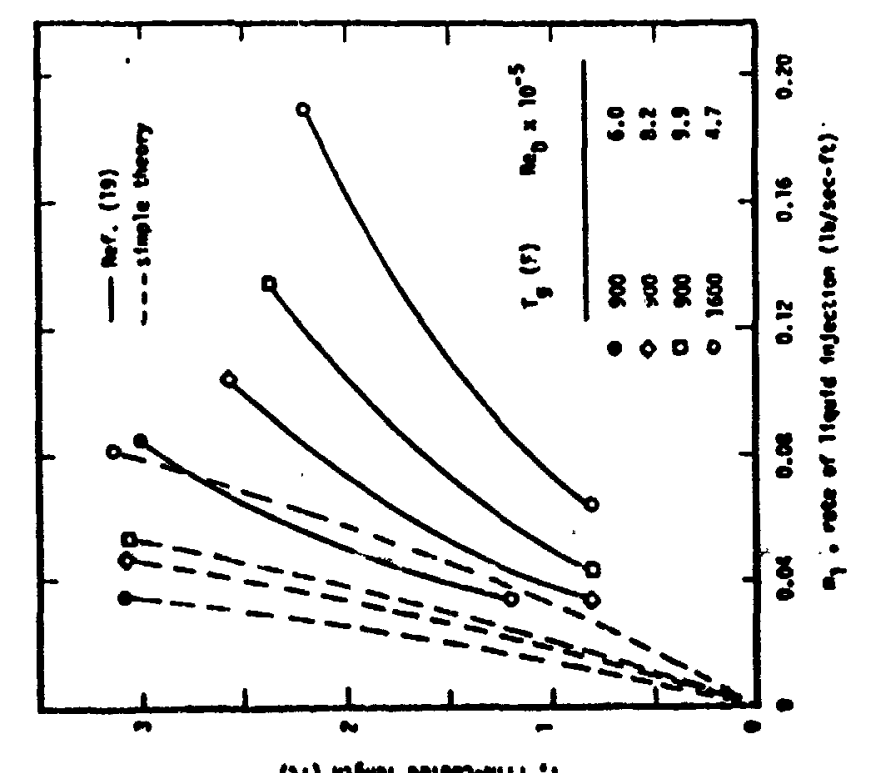

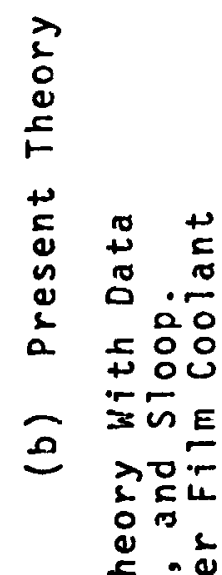

至

员些

+ E

a

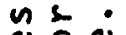

造告

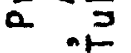

ट्丶े

$\approx=0$

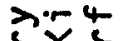

है

Ex

$\leftarrow$

음웅

告施

E。

ज4 4

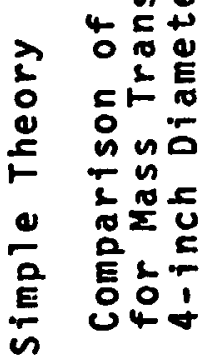

(II) ment parcosolis : 


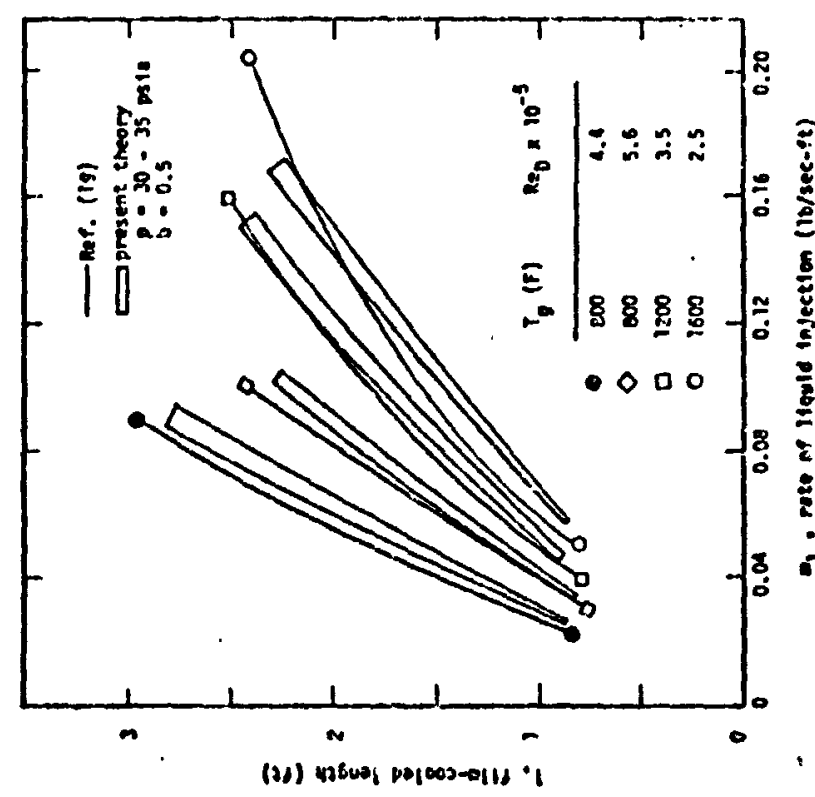

2
0
0
0
1
+
0
0
0
0
0
0
0
2
0

$\underset{0}{+\infty} \stackrel{+}{\infty}$

웅

어

उUE

त्रेष्ट

이

\&

$F E$

+ 我

E

\%

ฮิ

<造

a. 3

हैं

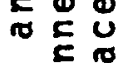

$>-5$

देख

\&

다

-

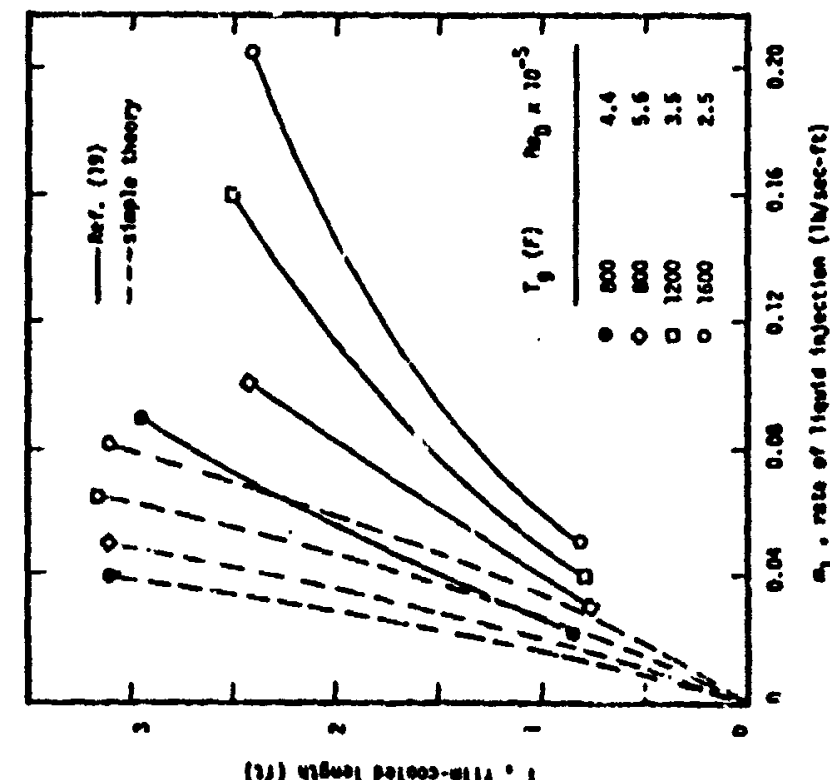

므유

题电

ज4

之)

도음

ins

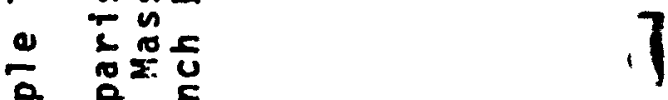

的 号它京

3

$\dot{m}$
$\dot{0}$
$\dot{L}$ 
The possible catastrophic consequences of designing a liquidfilm cooled system on the basis of the simple theory are evident.

Figures $37(b)$ and $38(b)$ present the resuliant computations for the present theory of mass transfer. Because the values for the gas stream pressure were not indicated explicitly for the data that were reported in Ref. (19), a band is shown for the present theory for each set of experimental data. For the two-incin diameter duct, $p$ was assumed to vary from 30 to $35 \mathrm{psia}$. An.. f the 4-inch diameter duct, $p$ was assumed to vary from 25 to $30 \mathrm{psia}$. The larger values for $\mathrm{m}_{1}$ at any particular value of 1 correspond to the lower values for pressure. (For the same value of gas stream Reynolds number, or gas mass velocity $G$, the value for the momentum parameter $M_{0}$ increases with decreasing pressure. And the amount of entraired liquid increases with increasing $\left.M_{0}.\right)$ A value of 0.5 for the parameter $b$ in Eq. (3-64) was employed to compute the results that are shown in Figs. $37(b)$ and 38(b) for the smooth-surface ducts. Recall that the simple theory presented in Subsection 3.2 .2 for the extension of the results of the present investigation to an arbitrary film cooled length showed that for the classical case of turbulent boundary layer flow over a flat plate, a value of approximately 0.6 for the parameter b was predicted. That value for $b$ resulted in predicted mass transfer rates that were generally 10 percent greater than the calculated 
results that are shown in Figs. $37(b)$ and $38(b)$. However, as discussed earlier in section 4.1 , that agreenent betwcen the predicted and the experimental results is probably within the accuracy of the experimental data.

Comparison of Figs. $37(b)$ and $38(b)$ shows that the agreement between the experimental results and the results calculated from the present throry is somewhat better for the 4-inch diameter. smooth-surface duct than it is for the 2-inch diameter duct. However, at least two reasons can be suggested as to why the experimental data for the 4-iach diameter duct should be weighted moie heavily in an evalwation of the present theory: (a) the gas flow in the 4-inch diameter duct should more closely correspond to the case of boundary layer flow over a flat plate*, and more importantly, (b) it was stated in the subject reference that the agreement between the data for any two experiments conducted at the same flow conditions was generally much poorer for the experimerts with the 2-inch diameter duct than it was for the 4-inch diameter ducts.

Figure 39 presents a similar comparison between the calculated results and the experimental results for the 4-inch diameter, rough-surface tube. The solid curves shown in the figure represent the data that are presented in Fig. 6(c) of Ref. (19). Approximately 15 datun points are represented by each of the solid curves.

* See assumption (a) in Subsection 4.3.3. 


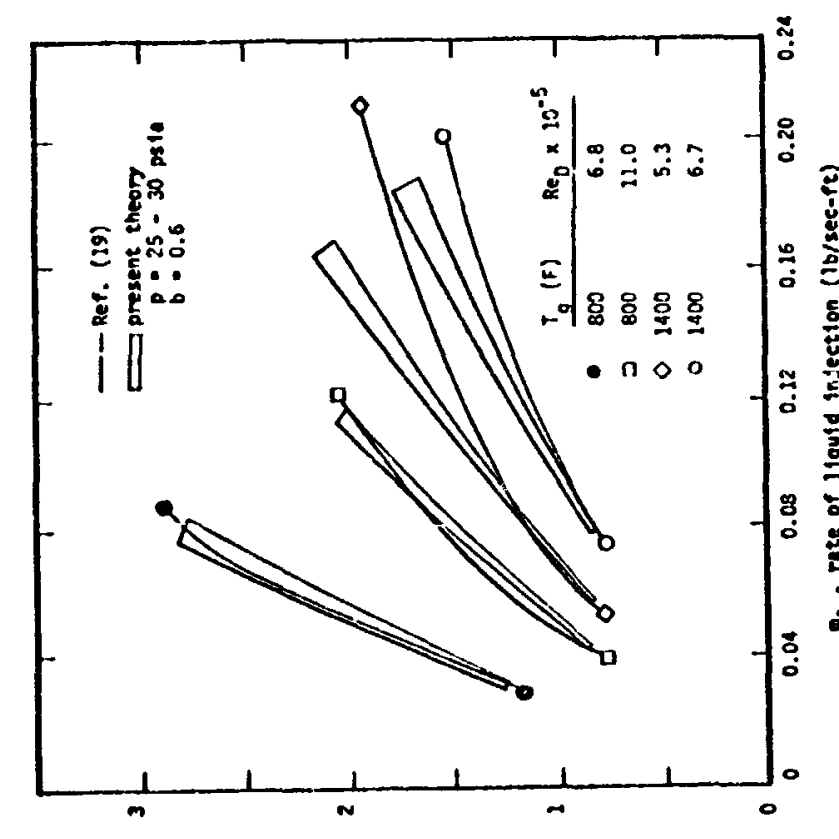

(2) yzbual palooj-wtH

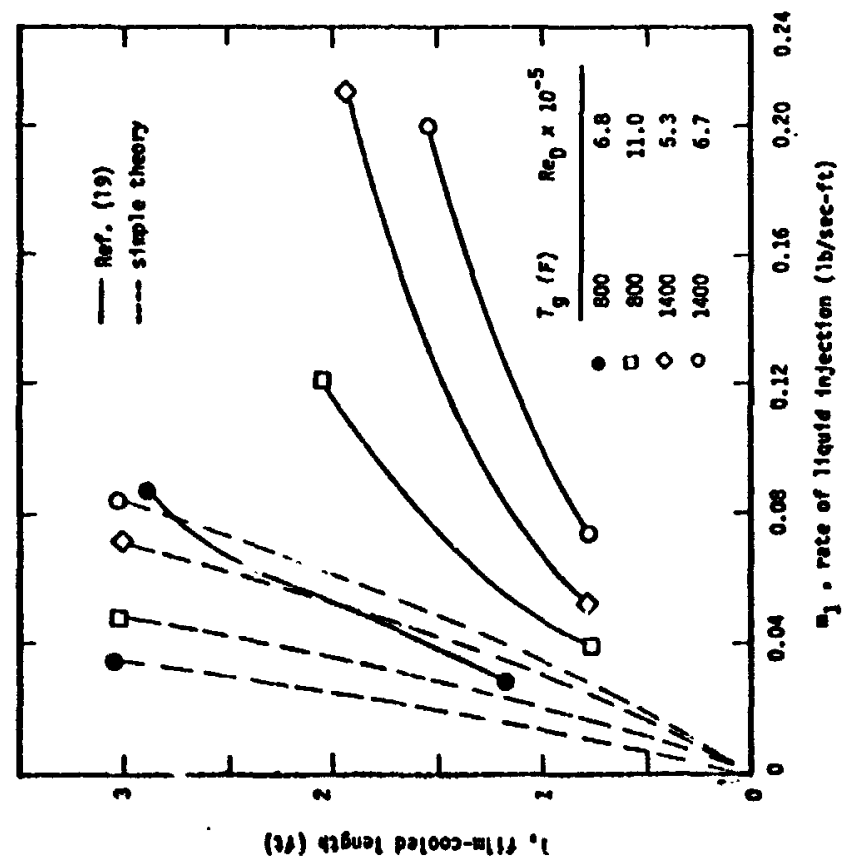

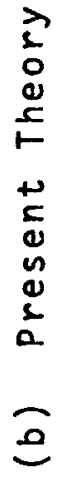

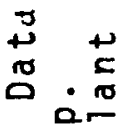

등ㅇㅇㅇ

$\div \frac{0}{3}$

究它至

$\Phi$

F章

$+\stackrel{2}{*}$

结

is

ปे

\&过

a.

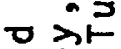

正。

ช气

$>\approx$

㲅文

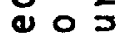

Ex

ข

옴

을

Eิ

ज4:

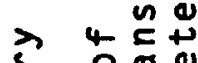

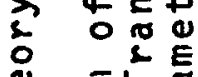

\&

$\mp \quad$ in

(1)

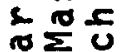

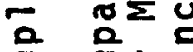

E Eั5:

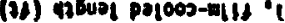

$\approx$ \%

$\dot{\sim}$ 
Figure $39(a)$ again shows the poor agreement between the experimental results and the results that were computed from the simple theory for mass transfer. The mass transfer rates that were realized experimentally are shown in Fig. 39 (a) to be as much as 400 percent greater than those predicted by the simple theory.

The computed results shown in Fig. $39(\mathrm{~b})$ for the present theory were calculated from Eq. (3-64) with a value of 0.6 for the parameter $b$. The band that is presented for each set of experimental data represents a variation in the static pressure of 25 to $30 \mathrm{psia}$. In general, the agreement between the experimental data and the results that are calcu. lated from the present theory is satisfactory. The greatest difference orcurs for the experimental data at the larger rates of liquid injection, $m_{1}$. However, even for those data, the present theory is in agreement to within the reported accuracy of the experimental data.

The following conclusion is drawn from the foregoing comparison of the computed results of the present theory with the experimental data of Ref. (19) for both the smoothsurface ducts and the rough-surface duct: to perform conservative engineering calculations so as to allow for unknnwn factors such as the physical condition of the wetted surface, the value of 0.6 for the parameter b in Eq. (3-64) 
should be employed*. That conclusion is in good agreement with the result that was predicted by the analysis of subsection 3.2.1 and Subsection 3.2.2.

4.3.5 A Comment on the Presentation of Data for Liquid-Film Cooling in Terms of Dimensionless Parameters

Consider the presentation of the experimental data of Ref. (19) in terms of a Nusselt number-Reynolds number type of plot. The effective** average Nusselt number, denoted by $\mathrm{Nu}_{e}$, can be calculated from the following expression. Thus

$$
N u_{e}=\frac{m_{1} / l}{G} \frac{R e_{D} P r}{B_{h}}
$$

Figure 12 of Ref. (19) presents plots of $\mathrm{Nu}$ as a function of $R_{D}$ showing some of the experimental data for both the smooth-surface tubes and the rough-surface tube. Figures 40 and 41 of the present report show a comparison between those data and the results that are calculated from the

* Note that this conclusion applies to only that case where the gas stream velocity is essentially constant over the length of the liquid film.

**The term "effective" is introduced because $\mathrm{Nu}$ includes the mass transfer due to entrainment in addition to that due to evaporation. 


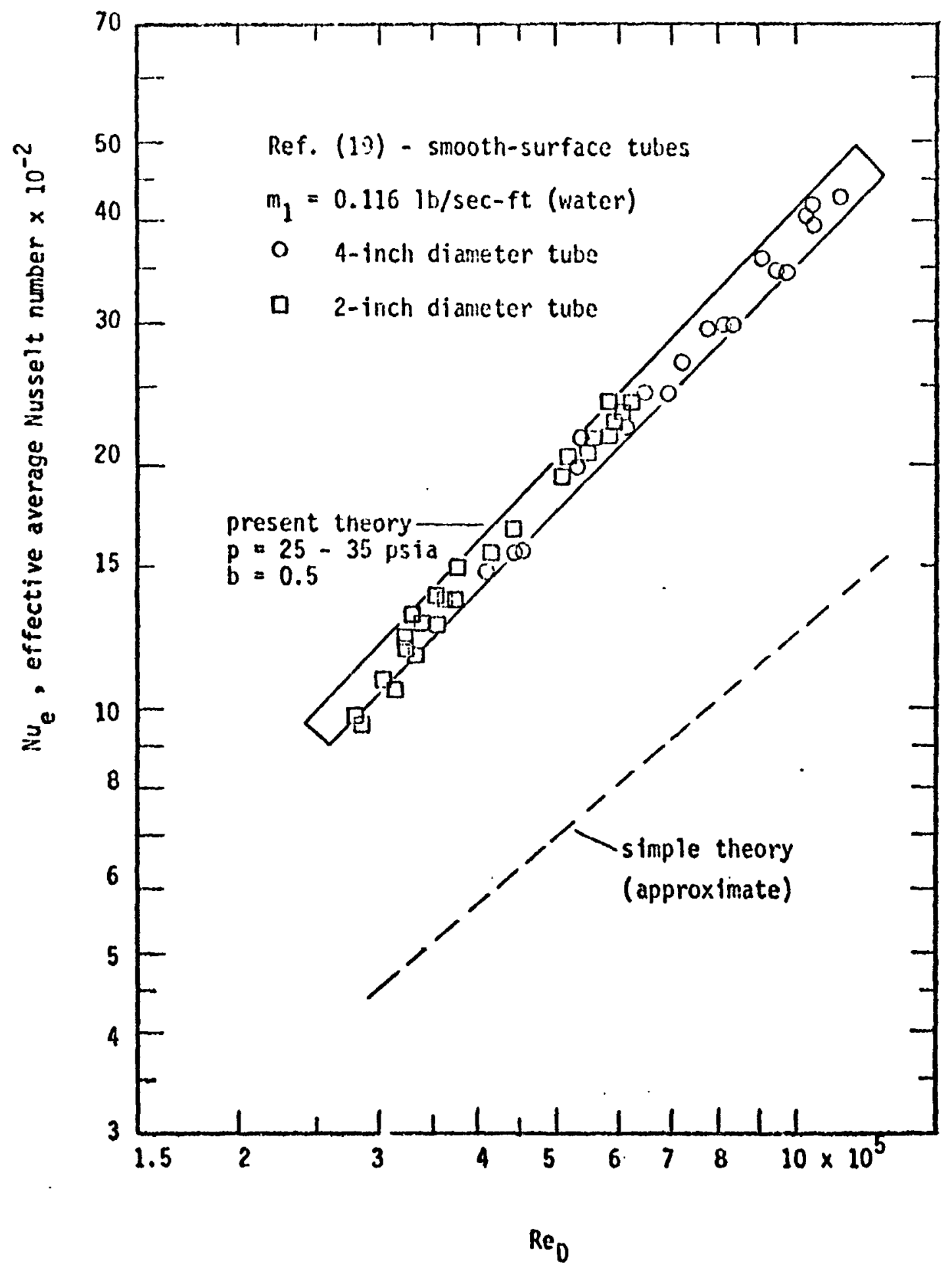

Fig. 40. Nusselt-Reynolds Number Plot of Data Due to Kinney, Abramson, and Sloop. Smocth-Surface Tubes 
I.

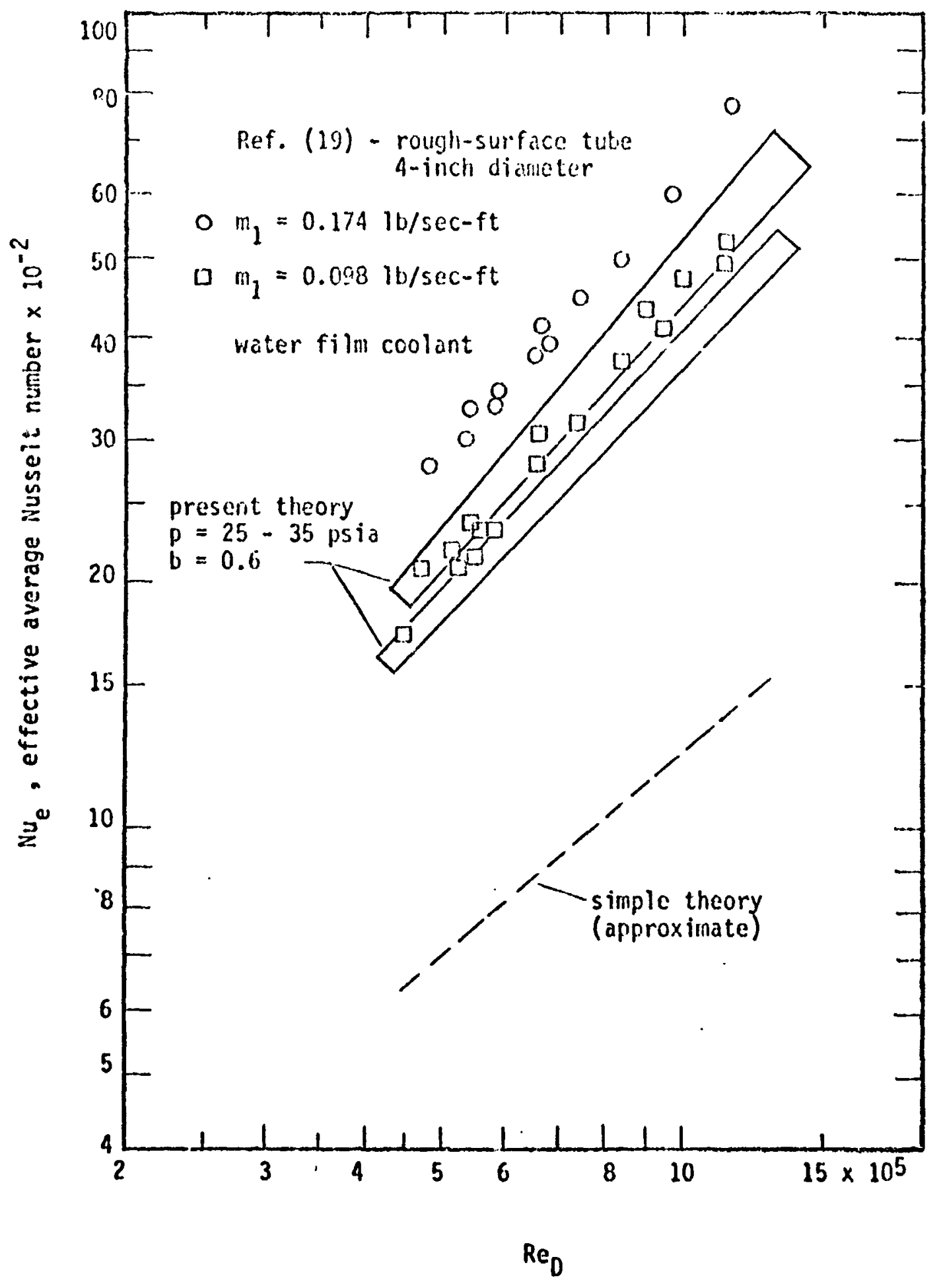

Fig. 41. Nusselt-Reynolds Number Plot of Data Due to Kinney, Abramson, and Sloop. Rough-Surface Tube 
present theory*. Figure 40 shows the results for the smooth-surface ducts, and Figure 4l. presents the results for the rough-surface duct. Note that each set of experimental data is characterized by a certain value for the rate of liquid injection, $m_{1}$; the values for $m_{1}$ are listed in the figure. The interrupted curve shown in both Fig. 40 and Fig. 41 corresponds to the case of simple mass transfer, where the varicus "F" correction factors (see subsection 3.1.2) were employed in the evaluation of the averuge Nusselt number. Because these correction factors are dependent on parameters other than the gas stream Reynolds number, the curves for simple-mass transfer are labeled "approximate". In evaluating the net mass transfer rate, $m_{1}$, a value of 0.5 for the parameter $b$ in Eq. (3-64) was utilized in the calculations for the smooth-surface ducts and a value of 0.6 was empluyed for the rough-surface duct.

*In the calculation of the Nusselt number in Pef. (19), the thermal conductivity for the gas stream, $\mathrm{k}_{\mathrm{g}}$, was introduced. However, the values for $k_{g}$ that were employed in those calculations were generally 10 percent to 15 percent greater than those listed, for example, in Ref. (40). To illustrate, Appendix $C$ of reference (19) gives a value for $k_{g}=15.6 \times 10^{\circ 6}(B t u / s e c-f t-F)$ for a gas stream temperature $T_{g}=2000 \mathrm{~F}$. But Ref. (40) gives a value for $k_{g}=13.1 \times 10^{-6}$ (Btu/sic-ft-F) at the same temperaiure. Therefore, to make some correction for this fact, the values fior Nue presented in Figs. 40 and 41 are greater than those presented in the original reference by a factor of 1.10 . 
The bands shown in Fig. 40 and Fig. 41 for the theory that was developed in the present report correspond to the gas stream pressure variation indicated in the figures. Figures 40 and 41 demonstrate again the poor agreement of the simple mass theory and the much more satisfactory agreement of the present theory with the experimental results. However, a more important point should be noted from the comparisons illustrated in the figures. Investigators of the problem of 1 iquid-film cooling have commonly presented their experimental data in the form of such a Nusselt-Reynolds number type of plot $(19,20,24)$. The experimental data of the present investigation, how ver, together with the data due to Kinney, et al. (19), show that this is generally a very poor way to present such experimental data because it does not account for the considerable importance that the parameters $M_{0}, \sigma$, and $m_{1}$ can have on the net rate of mass transfer from a thin liquid film to a high velocity gas stream. This type of data presentation can be very misleading for the practicing engineer who is concerned with the problem of designing a liquid-film cooled system, but who is not cognizant of the importance of the interfacial phenomena, particularly the entrainment phenomenon. It is hoped that the results of the experimental investigation reported herein clearly demonstrate the general importance of the interfacial phenomena in the calculation of the net rate of mass transfer from a thin liquid film to a hot gas.stream. 


\subsection{The Data Due to Fmmons:}

\subsection{Introductory Remarks}

Emmons (20) presents experimental data for liquid-film cooling of a cylindrical rocket motor combustion chamber. Experimental determinations were made of the effects of changes in the gas stream temperature, prossurc, and Reynolds number upon the required flow rate of a single liquid (water) needed to establish a range of im cooled lengths $(1=4$ to 8 inches). Experiments were also conducted with other coolants**, at one gas stream flow condition, to study the influence of the physical property values for the liquid on the rate of lass transfer from the liquid film to the gas stream. Most of the data presented in Ref. (20) were obtained for a gas stream temperature of $4100 \mathrm{R}$ and a chamber pressure of 500 psia. Only those data are reviewed herein.

Subsection 4.4.2, which follows, describes the basic experimental apparatus that was employed by Emmons. The primiary assumptions employed in the calculation of the mass transfer rates from the theory of the present report are discussed in subscction 4.4.3, and the comparison is shown in Subsection 4.4 .4 between the measured and the predicted results.

* See table 3 for the nominal flow parameters for these data.

**anhydrous anmonia, ethyl alcohol, and Freon-113 


\subsubsection{The Basic Apparatus}

Figure 42 illustrates schematically the basic experimental apparatus employed by Emmons in his study of liquidfilm cooling. The experimental rocket motor burned gaseous hydrogen and air as the propellants. The inside diameter of the chamber was 3.14 inches. Tro convectively cooled cylindrical sections were installed upstream of the adiabatic film cooled test section. The first convectively cooled chamber was 9.42 inches long and the second convectively cooled chamber was 1.48 inches long. Emmons utilized the 1.48-inch long section to calorimetrically estimate the hea: transfer to the dry wall immediately upstream of the film cooled test section. An observation will be made in Subsection 4.4 .5 regarding the measurements that were obtained for that 1.48 -inch long section.

The liquid coolant was injected through $72 \mathrm{~V}$-shaped grooves that were milled into the downstream face of the film coolant injector. The liquid was introduced onto the test surface with a component of velocity in the tangential, or circumferential, direction.

\subsubsection{The Assumptions}

The primary assumptions employed to analyze the experimental data of ket. (20) are summarized as follows:

(a) The gas flow was treated as boundary-?ayer type f?ow over a flat plate. The bourdary layer was assumed to be 


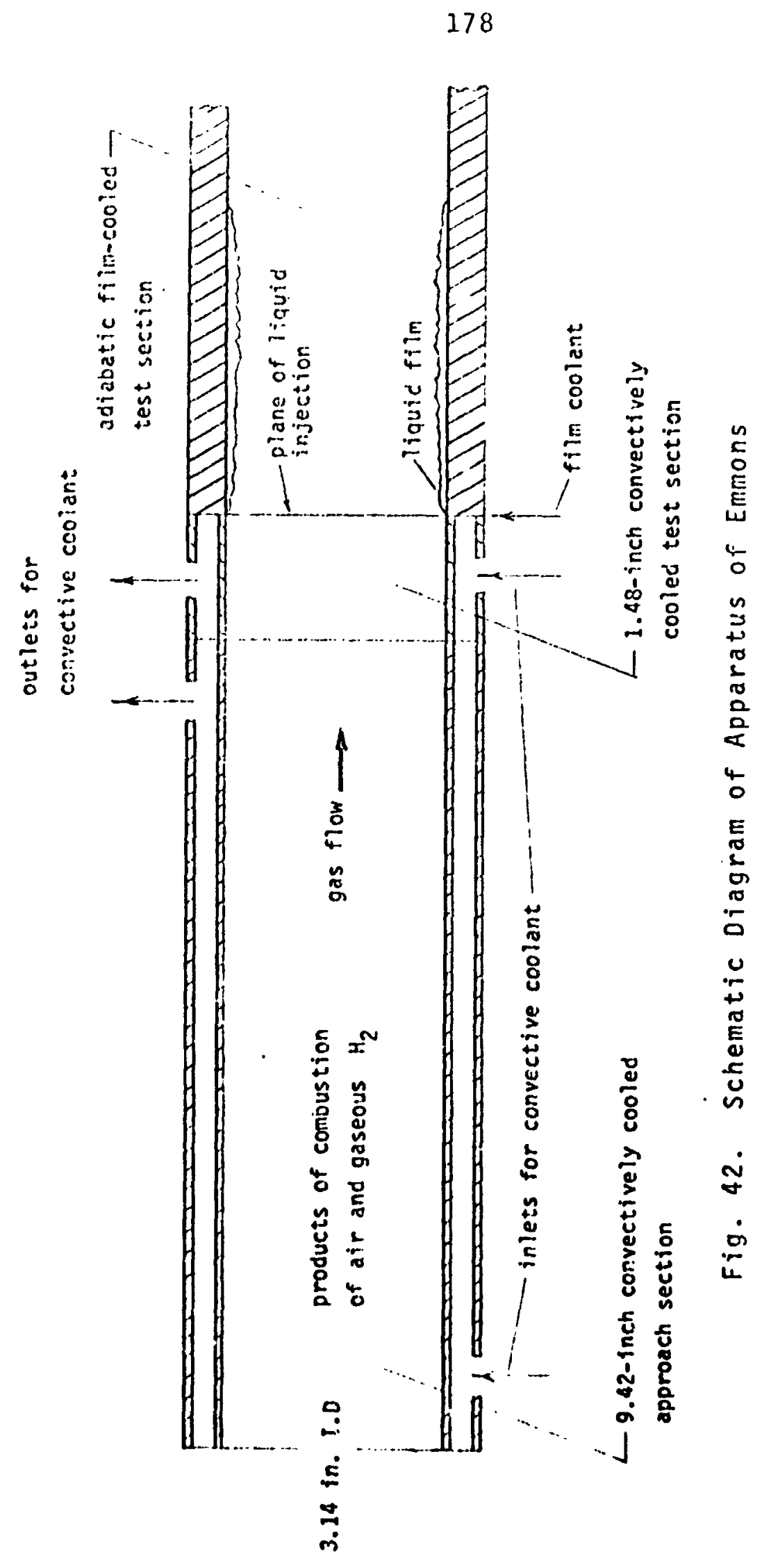


fully turbulent and to develop from the leading edge of the 9.42-inch long convectively cooled section.

(b) The temporature for the liquid film at the point of injection was much less than the wall temperature immediately upstreall so that a thermal entrance length $x_{t}=9.42+1.48=$ 10.90 inches existel.

(c) No chemical reactions occurred between the vapor for the film coolant and the main stream gas within the boundary layer that developed over the liquid film.

(d) The value for the energy balance parameter $\phi$ was calculated from Eq. $(4-1)$. The computed values for $\phi$ are shown in Table 5 below.

(e) Radiation heat transfer was neglected.

(f) The properties of the gas stream were estimated from the results presented by Rannie in Ref. (43)*. For a gas stream temperature of $4100 \mathrm{R}$ and a chamber pressure of 500 psia, the pertinent thermodyuamic properties that were estimated from Ref. (43) are as follows:

$$
\begin{aligned}
& M_{g}=23.0 \mathrm{lb} / 1 \mathrm{~b}-\mathrm{mole} \\
& c_{p g}=0.43 \mathrm{Btu} / 1 \mathrm{~b}-\mathrm{R} \\
& \gamma=1.255 \\
& \mu_{g}=4.75 \times 10^{-5} \mathrm{lb} / \mathrm{ft}-\mathrm{sec}
\end{aligned}
$$

*Emmons (20) docs not list the values that he assumed for the thermodynamic properties of the hot gas stream. 
(g) The reference temperature for the evaluation of the surface tension o was estimated from the procedure that was suggested in section 4.2. Table 5 presents the va?ues for the pertinent parameters thai entered into that evaluation, together with the computed values for $T_{1, r}$ and 0 . Also listed in Table 5, for completeness, are the values for tha heat transfar parameter $B_{h}$ and the interfacial vapor concontration $C_{s}$. $B_{h}$ was calculated from the right-hand side of Eq. $(3-87 b)$, and $C_{s}$ Was calculated fromi Eq. $(3-26) *$.

of the aforementioned as sumptions, Assumption (3) that the heat transfer due to radiation is negligible is probably one of the ieast valid. The products of combustion of air and gaseous hydrogen contain a substatial qualitity of water vapor wilich is a strong radiator, particliarly at the temperature level of $4100 \mathrm{~F}$. Perhaps 25 perceni or more of the net heat flux to the surface of the liquid film was due to radiation heat transfer. An attempt was not mada, however, to estimate that radiation heat transfer. Siynificant conclusions can be drawn about the usefulness of the theory developed in the present report for the prediction of the rate of mass transfer fror a liquid film to a hot, high velocity gas stream without trying to solve that complex problem

*Appendix 0.2 presents $H_{v}, P_{v, s}$, and $\sigma$ as a function of the liquid temperature for the liquids that were investigated by Emmons. 


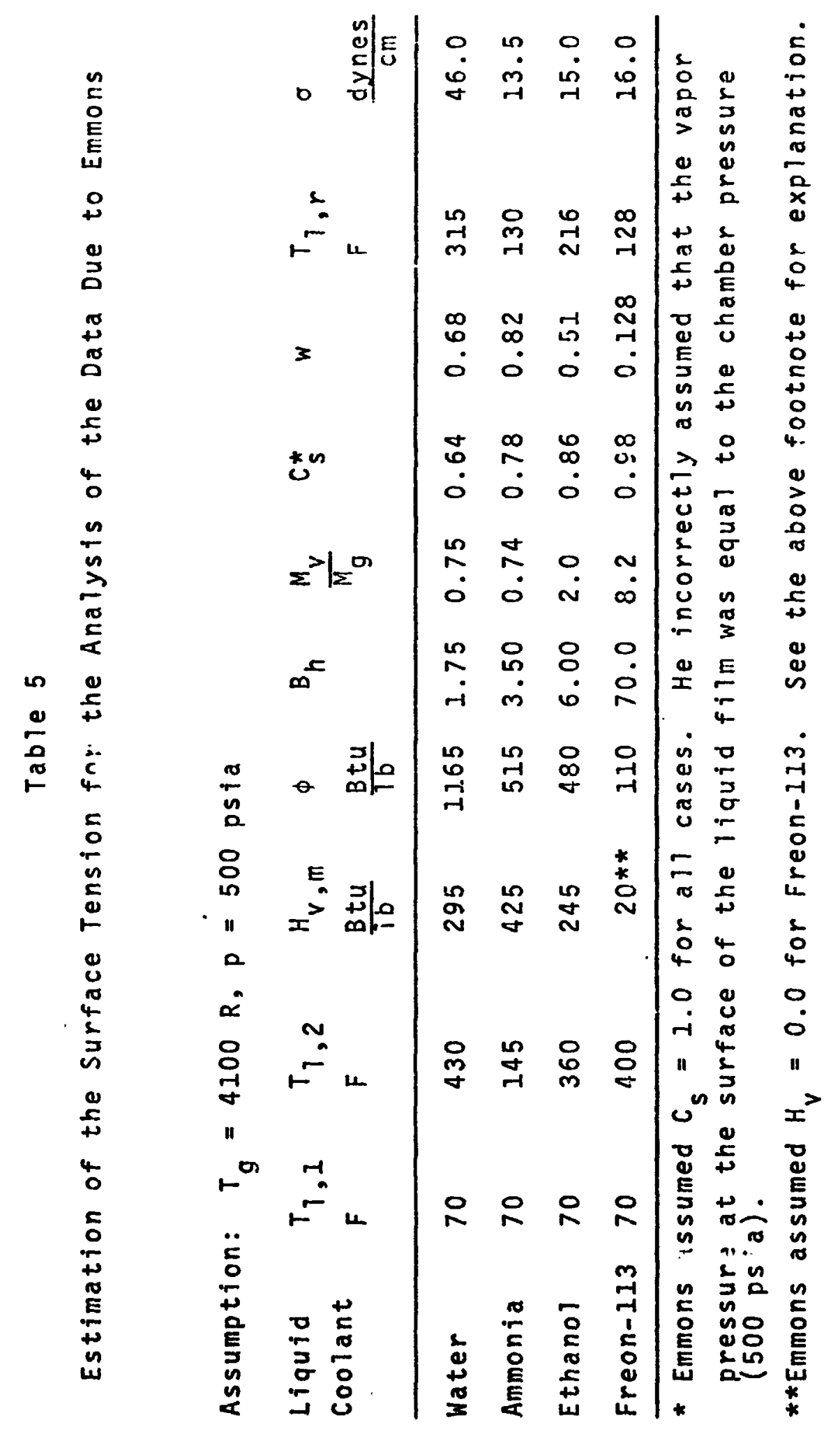




\subsubsection{Comparison of the Present Theory With the Experimental Data}

The aforementioned assumptions were employed in conjunction with the primary equation (3-64) to analyze the data of Ref. (20). The results of those computations are summarized in the following paragraphs.

Figure 43 shows the comparison of the computed results and the experimental data for the case where the gas stream Reynolds number was a variable and water was the film coolant. The gas stream Reynolds numbers are listed on the figure. Part (a) of the figure presents a plot of the mass transfer rate that was predicted from the simple theory for mass transfer versus the measured mass transfer rate. Part (b) of Fig. 43 then shows the comparison between the experimental data and the present theory. A value of 0.6 for the parameter b in Eq. (3-64) was employed to compute the results for the present theory.

Figure 43(b) shows that the present theory for mass transfer predicts a mass transfer rate that is consistently less than that realized axperimentally. However, Fig. 43 also shows that the present theory is a substantial improvement over the simple-theory for mass transfer. Moreover, if the radiation heat transfer was accounted for, the agreement between the present theory and the experimental data would be improved. Indeed, if one also accounts for the likelihood of considerable experimental error in the 


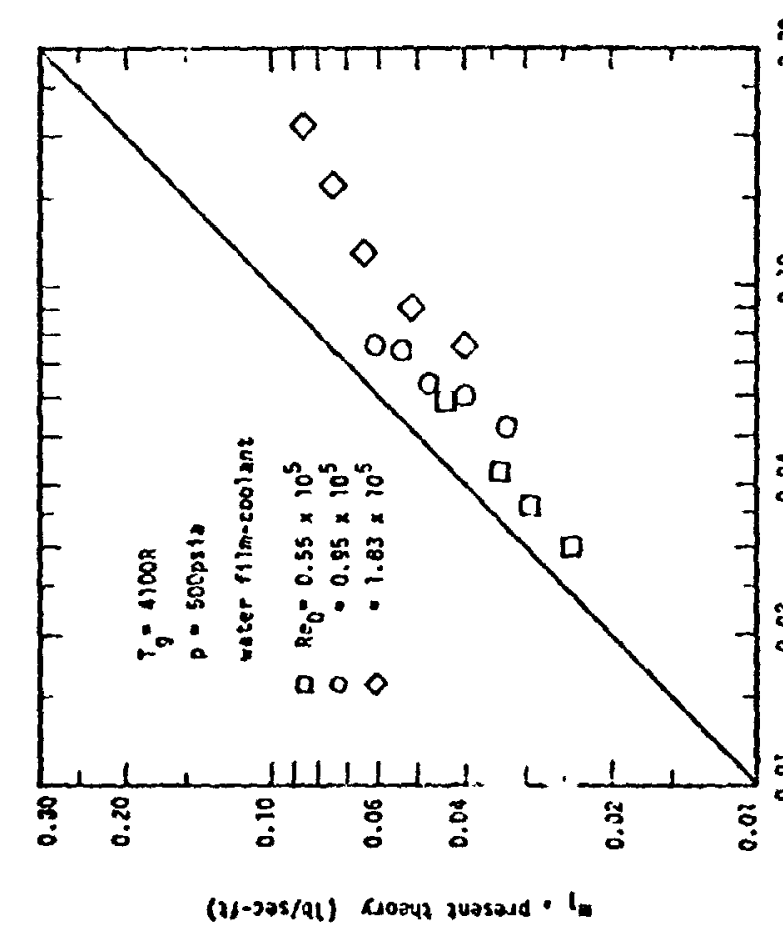

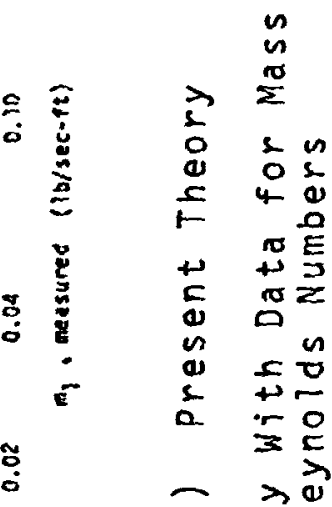

I

I

E

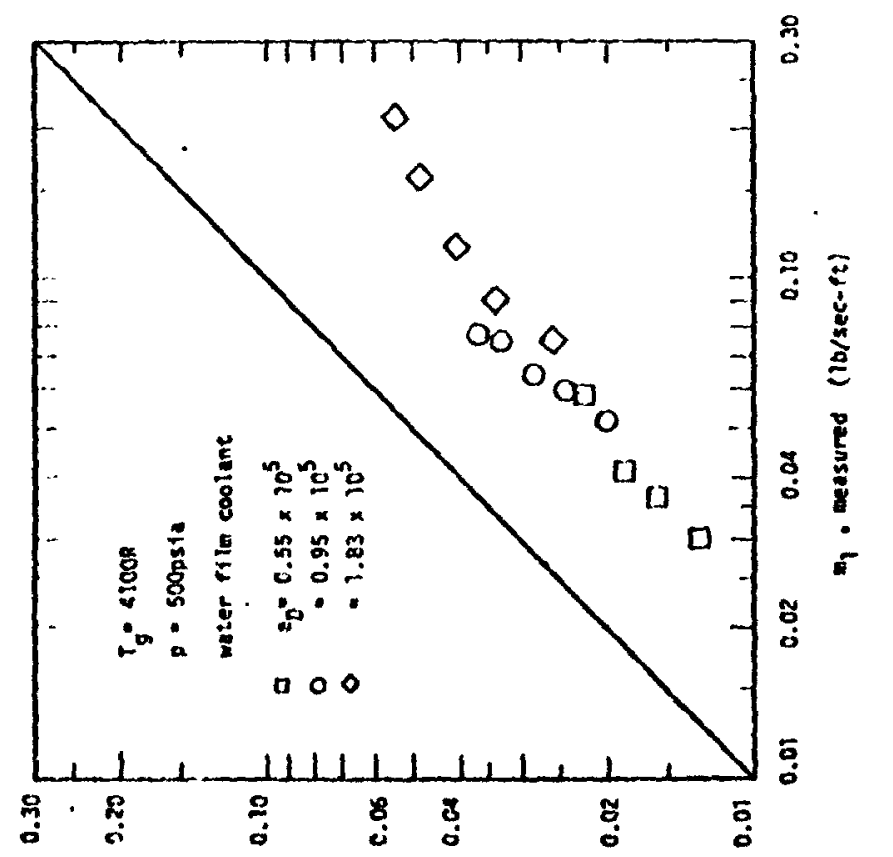

$D$

is

d

市京

as

an

\&

$a$

웡

I

$\pi$

ते

i

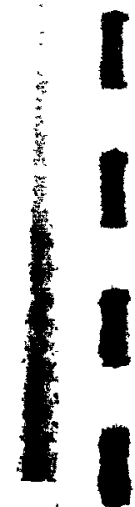

용

E.

농

을

的。

4
0
0

$F \quad 0$

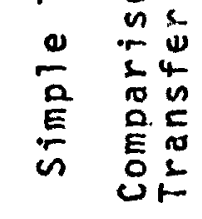

๓

$\dot{v}$
$\dot{\sigma}$

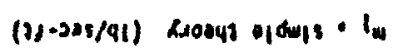


reported datax, it could probably be argued that the agree.ment between the present theory and the reported experimental data is quite satisfactory.

Figure 44 presents a similar comparison for the data that were obtained for the film coolants water, ammonia, ethanol, and Freon-113 at a gas streall Reynolds number of $0.68 \times 10^{5}$. Again, part (a) of the figure compares the experimental data for mass transfer with the mass transfer that was predicted from the simple theory; and part (b) of Fig. 44 shows the comparison between the theory that was developed herein and the reported data. Figure 44 demonstrates further that the proposed correlation for mass transfer is an improvement over the simple theory. If the neglect of the radiation heat transfer and the probable experimental error is taken into consideration, the agreement between the present theory and the experimental data shown in Fig. 44 is quite satisfactory.

\subsubsection{An Additional Comment of the Research Due to Emmons}

It was indicated in Subsection 4.4 .2 that the 1.48 -inch long convectively cooled section** (just upstream of the film cooled test section) was employed to determine the rate of heat transfer to a ciry wall as a function of lice pertinent 

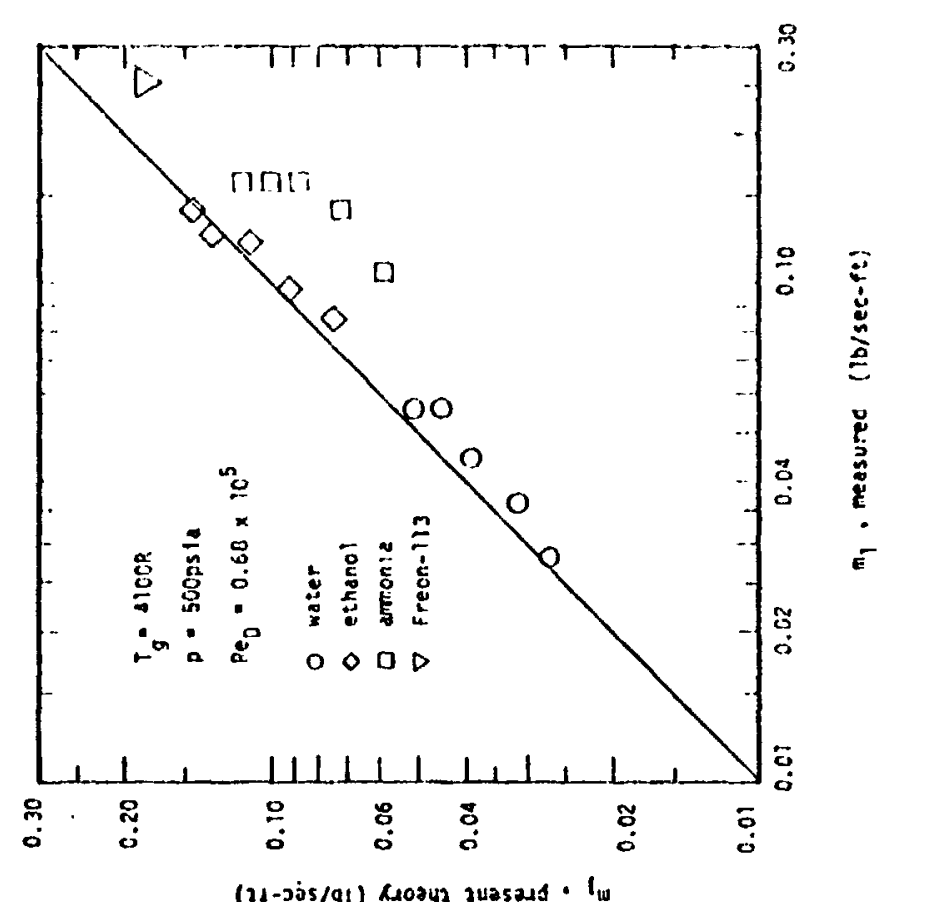

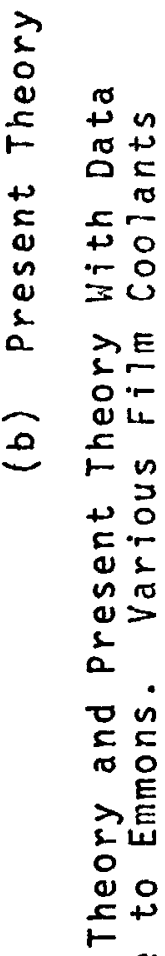

[

음

E

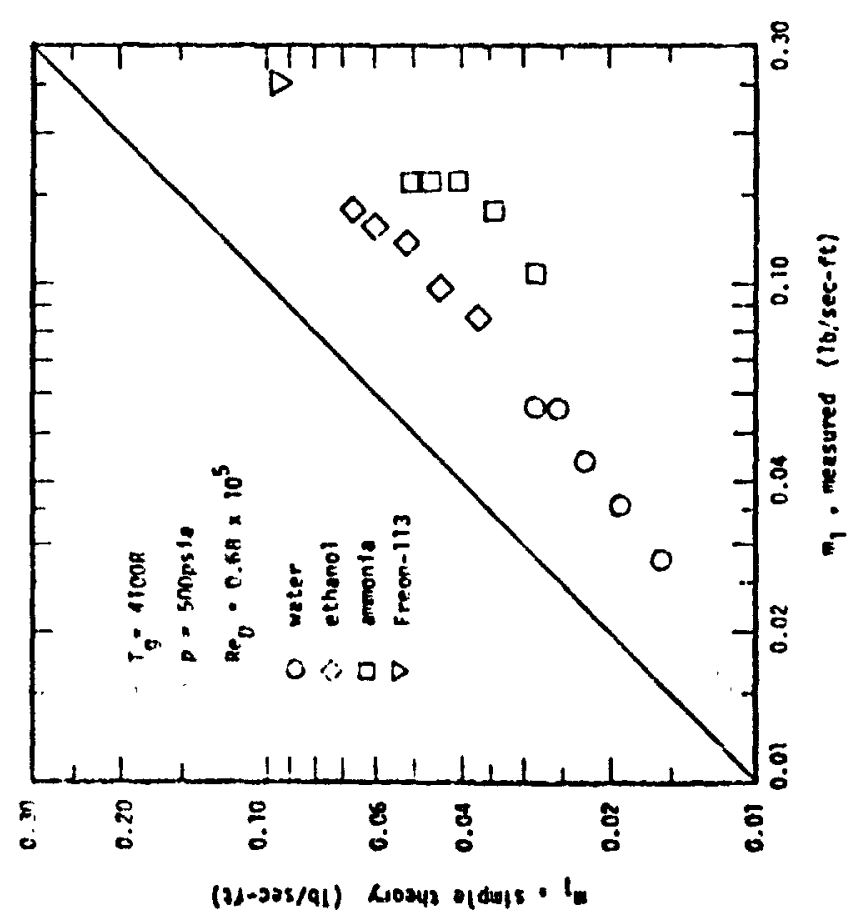

E.

is

$4 \stackrel{\text { E }}{0}$

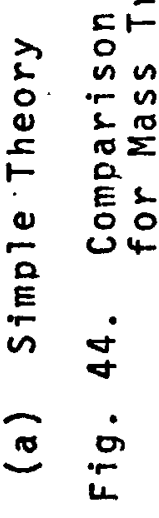

I

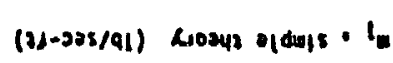


gas strean flow conditions. Emmons compared those dry-vall measurements to the measurements he obtained for the 11 quid$f 11 \mathrm{~m}$ cooled wall region and concluded that the rate of heat transfer to the $14 q u i d f\{1 m$ was less than the rate of heat transfer to the dry wall for the same gas stream conditions. In essence, he concluded that the analogy between simplemass-transfer cooling and 11 quid-film cooling was valid. However, the experimental data reported herefin, together with those reported by kinney, et al. (19), show rather conclusively that the analogy between the simple-mass transfer process and the process of 11 quid-f11m cooling is generally very poor. In fact, the computed resuits shown in Fig. 43 and $\mathrm{Fig} .44$ suggest that the experimental data reported by Emmons do not support the analogy. This apparent discrepancy is discussed in the following paragraph.

Emmons found that the dry-wall heat transfer data could be correlated by a Nusselt-Reynolds number type of correlation that was suggested by Humble, et al. (44) for fullydeveloped turbulent tube flow. That particular corre. lation, however, speciffes that the properties for the gas that enter into both the Nusselt number and the Reynolds number be evaluated at the wall temperature. The evaluation of the properties at the wall temperature is a cominon procedure employed to correlate heat transfer data for the case where heat is transferred from the wall to the gas stream (36) (1.e., the wall is at a higher temperature thian the gas 
gas stroan flow conditions. Emmons comparad those drymall measurements to the moasuremonts he oblathed for the 1 gutd. flim cooled wall regiton and concluded that the rate of heat transter to the liquid filin was lass than the rate of heat trangter to the dry wall for the same gas strean condition. in essence, he concluded that the andogy betiwen slimplew inassutranstor cooling and Tlquideftlin cooling was valid. However, the experimental data reported horefri, together with those reported by kinney, at al. (19), show rather conm clusively that the analogy between the simplemas transfor process and the process of liquidwitim cooling is garerally very poor. In fact, the computed results shown in Fig. 43 and fig. 44 suggest that the experfinental data roported by Emmons do not support the analogy. This apparent discrep. ancy is discussed in the following paragraph.

limons found that the dryawall heat trafister data could be coprelated by a Nusselt-Reynolds number type of correm lation that was suggested by Humble, et a 1 . (44) for fullydeveloped turbulent tube flow. That particular correc lation, however, specilfes that the properties for the gas that enter into both the Nusselt number and the Reyrolds number be evaluated at the wall temperature. The evaluation A the properties at the wall tomperature is w whon prom ceduro employed to correlate heat tratisfer data for the case where toet is trangrempod from the wall to the gas strean (36) (1.6., the wall is at a highar temperature than the gos 
stream). That was the case that was investigated by Humble, et a 1. (44). However, for the case where the heat transfer is from the gas stream to the wall, as is the case for the data reported by chinos, the standard procedure is to evaluate the physical properties for the gas at some intermediate temperature between that for the gas stream and that for the wall. The choice of the reference temperature for the evaluation of the properties for the gas can be very important. To illustrate, Kays (36) suggests that the Nusselt number for the case of turbulent heat transfer from the wall to the gas stream is greater than the Nusselt number for the case of turbulent heat transfer from the gas stream to the wall, all other conditions being equal, by the factor $\left(\mathrm{I}_{\mathrm{g}} / \mathrm{T}_{\mathrm{s}}\right)^{0.25}$. For the dry-wall experiments of Emmons, a characteristic value of $\left(T_{g} / T_{s}\right)$ would be 4 with the resulting value for that factor being 1.41. It would appear, therefore, that the heat transfer rates that were realized for the dry-wall test section were approximately 40 percent greater than those that would be predicted by a standard correlation for fully developed, turbulent gas flow with the direction of heat transfer being from the gas to the wall of the tube. Part of that excess heat transfer could probably be attributed to radiation heat transfer. More significantly, however, it can be argued that part (or perhaps most) of that excess heat transfer was probably 
due to the fact that the assumption of fully-developed tube flow was a poor approximation to the flow over the 1.48-inch convectively cooled test section, particularly as regards the thermal flow field. Reference to the schematic illustration of the experimental apparatus presented in Fig. 42 shows that for the 9.42-inch long convectively cooled section, the external coolant (water) was introduced at the upstream end. It follows, therefore, that because of the heat transfer from the combustion chamber to the external coolant, both the temperature for the wall and for the coolant would increase in the flow direction until the coolant was withdrawn at a point just upstream of the 1.48 -inch long convectively cooled test section. Assuming that the temperature of the external coolant was the same at the inlet to the 9.42-inch long section and at the inlet to the 1.48-inch long section, it then can be argued that there existed a decrease in wall temperature (that could have been substantial) in going from the downstream end of the 9.42-inch section to the 1.48-inch section. Such a change in wall temperature would cause the rate of heat transfer to the 1.48-inch section to be significantly higher, and it makes the assumption of a fully-developed thermal flow field much poorer, than if the wall temperature was everywhere isothermal. Emmons did not attempt to determine what the pertinent thermal bounciary conditions were. Thus the validity of the above argument cannot be investigated further. 
The argument is presented here solely as a possible explanation for the apparent high rates of heat transfer that were measured for the dry-wall test secticn. It is unfortunate, however, that Emmons arrived at such an important conclusion that the liquid-film cooling process and the process of simple-mass transfer were analogous without having considered the interpretation of the dry-wall heat transfer data in greater detail. Also it will be shown in f.spendix A.4 of the present report that the major conclusion that Emmons drew with reference to his experimental data for the gas-vapor cooled region immediately downstream of the terminus of the liquid film is open to some question lecause of the possible incomplete interpretation of the experimental data for the dry-wall heat transfer experiments.

\subsection{The Data Due to Hermann, Leitinger, and Melnik*}

\subsubsection{Introductory Remarks}

Hermann, et al. (24) experimentally investigated the feasibility of liquid-film cooling the nozzle of a hypersonic wind tunnel. For the preliminary experiments reported in the subject reference, the film cooling of a Mach number 1.92 axi-symmetric Laval nozzie was considered. They were not primarily interested in obtaining quantitative data on the rate of mass transfer from a liquid film to an accelerating gas stream. In fact, only three datum points are 
presented that can be employed to evaluate the present theory. Moreover, the accuracy of these data points is questionable, primarily due to the fact that the instrumentation was limited. Specifically, oniy 15 thermocouples that were welded to the external surface of the film cooled wall were utilized to determine the average film cooled length. The thermocouples were distributed both axially along and circumferentially around the nozzle. Only 9 of the 15 thermocouples were installed downstream of the throat of the nozzle where the liquid film usually terminated. Thus, due to the ron-uniform way in which the liquid film usually terminates, it would be impossible, in general, to accurately determine the actual wetted area (or average film cooled length) from only 9 thermocouple leadings. However, in spite of the foregoing limitations, it is still of some interest to compare the reported data for mass transfer with the mass transfer that is predicted by the present theory for the case of an accelerated flow.

Subsection 4.5 .2 presents a discussion of the basic apparatus, and Subsection 4.5 .3 outlines the primary assumptions that were employed to analyze the experimental data. Subsection 4.5 .4 presents a comparison between the predicted and the experimental results.

To obtain some idea of the possible film coolant requirements for the cooling of the same axi-symmetric nozzle if it were installed on a high-energy propulsion device, 
Subsection 4.5 .5 presents an illustrative calculation based on the present theory for the case where the stagnation temperature $T_{\mathrm{g}}^{0}=4000 \mathrm{R}$ and the stagnation pressure $\mathrm{p}^{0}=$ 500 psia.

\subsubsection{The Basic Apparatus}

Figure 45 illustrates schematically the experimental apparatus of Ref. (24). The figure presents a Mach number 1.92, axi-symmetric Laval nozzle having a 3-inch long subsonic section and a 5 -inch long supersonic section. The diameter at the inlet to the nozzle was 8 inches; the diameter at the throat of the nozzle was approximately 2.7 inches; and the diameter at the exit of the nozzle was approximately 3.5 inches. ihe walls of the nozzle were essentially adiabatic.

A j-47 jet engine combustor was employed to produce the hot gas stream. The maximum stagnation temperature that was investigated was $1940 \mathrm{R}$.

The film coolant (water) was introduced at the inlet to the subsonic portion of the nozzle. The coolant was injected onto the walls of the nozzle through 48 holes that were evenly spaced around the circumference of the nozzle. For most of the investigation, the diameter of each hole was 0.011 inches. 


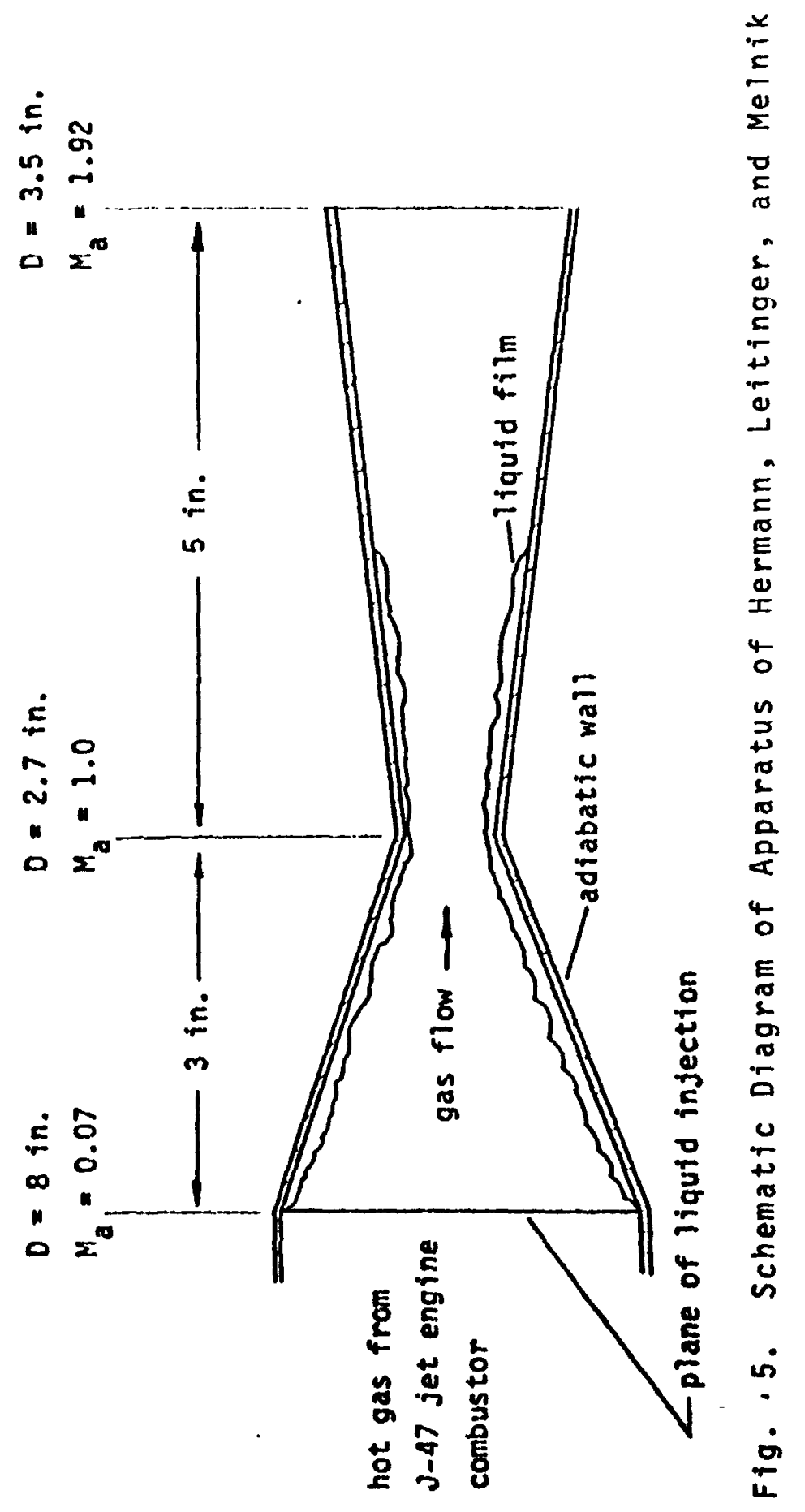




\subsubsection{The Assumptions}

The primary assumptions employed to analyze the experimental data of Ref. (24) are summarized as follows.

(a) The theory outlined in Subsection 3.3 .1 for predicting the rate of simple mass transfer from a liquid film to an accelerating gas stream was employed to determine the simple mass transfer rates for the subject case. The boundary layer was assumed to be fully turbulent and to develop from the leading edge of the 3 -inch long subsonic portion of the nozzle. In addition, the adiabatic-wall recovery temperature for the gas stream was assumed to be equal to the stagnation temperature $\mathrm{T}_{\mathbf{g}}^{0}$.

(b) Except for the thin boundary layer region next to the nozzle wall, the gas flow was assumed to be isentropic with a specific heat ratio of 1.33 . With that assumption, the values for $p, T_{g}$, and $u_{g}$ were computed as a function of the position along the axis of the nozzle. No corrections were made in the calculations for displacement effects.

(c) The thermodynamic properties for the gas stream were assumed to the same as those for dry air at the same temperature.

(d) The average temperature for the liquid film throughout the nozzle was estimated from Fig. 28 of Raf. (24) to be $220 \mathrm{~F}$ for the case of the three datum points that are analyzed herein.

(e) A value of $1000 \mathrm{Btu} / \mathrm{lb}$ for the energy ballance parameter 
$\phi$ was estimated from Eq. (4-1) with the substitution of the pertinent thermodynamic properties for water.

(f) Radiation heat transfer was neglected.

The foregoing assumptions were employed, together with the solution technique that was outlined in subsection 3.3.2 for the present theory, to analyze the subject experimental data. The value for the parameter $d$ in Eq. (3-74) that was employed in the calculations was 1.0. That value for $d$ was found to correlate the data for the present investigation for the case where the gas stream was accelerated over the wetted wall region.

\subsubsection{Comparison of the Present Theory With the Experimental Data}

Reference (24) presents, for three different gas stream conditions, the approximate film coolant flow rate required to wet the first 4 to 5 inches of the nozzle. The comparison between the experimental results and the results predicted from both the simple theory for mass transfer and from the present theory (assuming $d=1.0$ ) is as follows: Point 1: $T_{g}^{0}=1460 R, \quad p^{0}=69$ psia.

$$
\begin{array}{ll}
\text { experimental: } & m_{1}=0.037 \mathrm{lb} / \mathrm{sec} \\
\text { simple theory: } & m_{1}=0.017 \text { to } 0.021 \mathrm{lb} / \mathrm{sec} \\
\text { present theory: } & m_{1}=0.023 \text { to } 0.028 \mathrm{ib} / \mathrm{sec}
\end{array}
$$


Point $2: T_{g}^{0}=1470 R, \quad p^{0}=82$ psia.

$\begin{array}{ll}\text { experimental: } & m_{1}=0.037 \mathrm{lb} / \mathrm{sec} \\ \text { simple theory: } & m_{1}=0.019 \text { to } 0.23 \mathrm{lb} / \mathrm{sec} \\ \text { present theory: } & m_{1}=0.028 \text { to } 0.033 \mathrm{lb} / \mathrm{sec}\end{array}$

Point $3: \mathrm{T}_{\mathrm{g}}^{0}=1940 \mathrm{R}, \quad \mathrm{p}^{0}=82 \mathrm{psia}$.

experimental: $\quad m_{1}=0.0971 \mathrm{~b} / \mathrm{sec}$

simple theory: $\quad m_{1}=0.029$ to $0.036 \mathrm{lb} / \mathrm{sec}$

present theory: $\quad m_{1}=0.043$ to $0.053 \mathrm{lb} / \mathrm{sec}$

The foregoing results show that the agreement between the experimental and the predicted results is satisfactory for Point 2, is less satisfactory for Point 1 , and is rather poor for Point 3. However, for each point, the result predicted by the present theory is better than that predicted by the simple theory for mass transfer.

It is unfortunate that additionai experimental data were not presented in the subject reference in such a form that the present analysis could be further evaluated. Moreover, it is unfortunate that the accuracy of the three datum points that are reported is uncertain. One question that remains to be answered is to what degree (if any) compressibility effects for Mach numbers greater than, say, 0.5 alter the form of the correlation proposed herein for the rate of mass transfer from a thin liquid film to an accelerating, hot gas stream. 


\subsubsection{An Illustrative Example for $T_{g}^{0}=4000 R$ and $p^{0}=500$ psia}

To illustrate further the present theory, an example is presented in this section to show the film coolant requirements (predicted) for the cooling of the above axi-symmetric nozzle for the case where the gas flow has a stagnation temperature of $4000 \mathrm{R}$ and a stagnation pressure of $500 \mathrm{psia}$. These stagnation conditions are characteristis of the flow conditions that might be encountered in the appication of fillii cooling to thermally protect an exhaust nozzle of a high-energy propulsion device. This example serves to indicate the type of deviation from the simple theory for mass transfer that can be expected in the liquid-film cooling of such an exhaust nozzle.

The assumptions employed in the calculations are the same as those listed in Subsection 4.5.3. Again, the value of 1.0 for the parameter $d$ in Eq. (3-74) was employed in the computations.

Figure 46 presents a plot of the net rate of coolant injection as a function of the film cooled length of the exhaust nozzle (measured along the axis of the nozzle). The pertinent parameters that were employed in the calculations are summarized in the rigure. Tho interrupted curve shown in the figure represents the result that is predicted from the simple theory for mass transfer. The two solid curves represent the result that is predicted from the present 


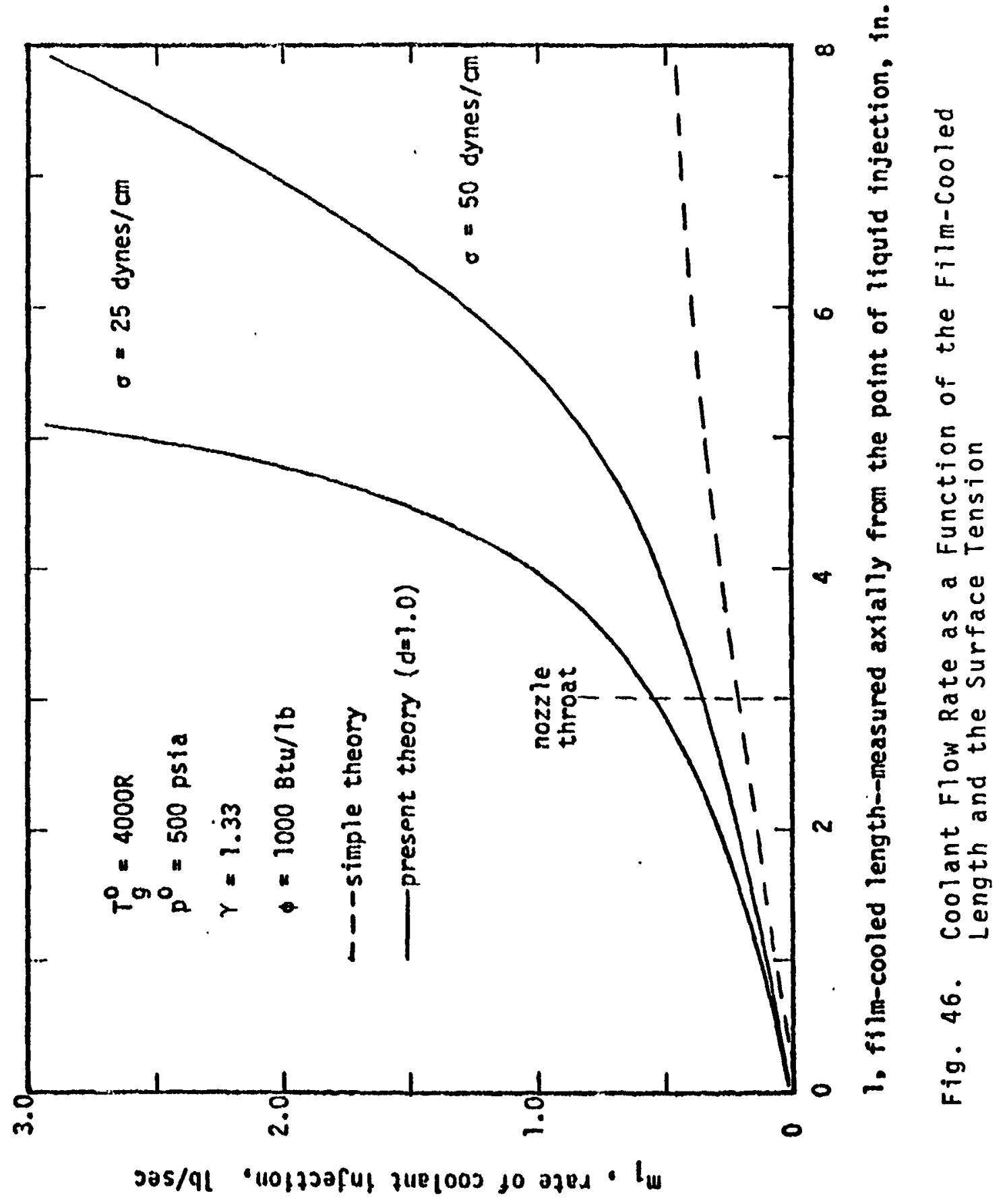


theory for two different values of surface tension $\sigma$. The larger value for $\sigma(50$ dynes $/ \mathrm{cm})$ represerits roughly the case where water is employed as the film coolant. Most of the other film coolants that might be of interest (e.g., alcohols, hydrocarbon fuels) are approximately represented by the smaller value for o $(25$ dynes $/ \mathrm{cm})$. Figure 46 shows that the present theory predicts, for the case where $\sigma=50$ dynes/cm, a coolant flow rate to cool the subsonic portion of the nozzle that is approximately 50 percent greater than that predicted from the simple theory. And, for the case where $\sigma=25$ dynes $/ \mathrm{cm}$, the present theory predicts an increase in the coolant requirements to cool the subsonic portion of the nozzle of 100 percent. More interestingly, however, Fig. 46 shows that to cnol both the subsonic and the supersonic portions of the nozzle, by injecting the coolant at only the leading edge of the subsonic portion, the coolant requi.ements predicted from the present theory become substantial. For the case where $\sigma=50$ dynes/cm, about 6 times more coolant is required to cool the entire nozzle than is predicted by the simple theory. For the case where $\sigma=25$ dynes $/ \mathrm{cm}$, the amount of coolant required to cool the entire nozzle is roughly 30 to 40 times that predicted by the simn'n theory. Although thesn praticted coolant flow rates are considerably greater than those predicted by the simple theory, the three datum points discussed in subsection 4.5 .4 suggest that the estimates from. 
the present theory nay even be low.

It is worthy of note that almost all of the difference between the results predicted by the simple theory and those predicted by the present theory is due to the entrainment phenomenon. That is, except for the very leading edge of the subsonic portion of the nozz?e where the gas stream velocity is relatively low, the roughness parameter $r$ is small, less than, say, 0.10 .

It also is interesting to note the local rates of mass transfer from the liquid film to the gas stream that are predicted by the present theory for the subjeci illustrative case. Figure 47 presents a plot of the predicted local rate of mass transfer from the liquid film into the gas stream as a function of the axial location throughout the nozzle. The pertinent parameters employed in the calculations are listed in the figure. Only the one value for surface tension of 50 dynes/cm was employed in the computations. The result that is predicted by the simple theory is indicated in Fig. 47. In addition, three curves are presented in the figure to fllustrate the result that is predicted by the present theory for three values of the net rate of coolant injection, $m_{l}$. The coolant flow rates shown are $0.5,1.2$, and $3.0 \mathrm{lb} / \mathrm{sec}$. From Fig. 46, those coolant flow rates correspond to predicted film cooled lengths of approximately 4,6 , and 8 inches, respectively. Figure 47111 ustrates that for all cases, the maximum value for the local rate of 


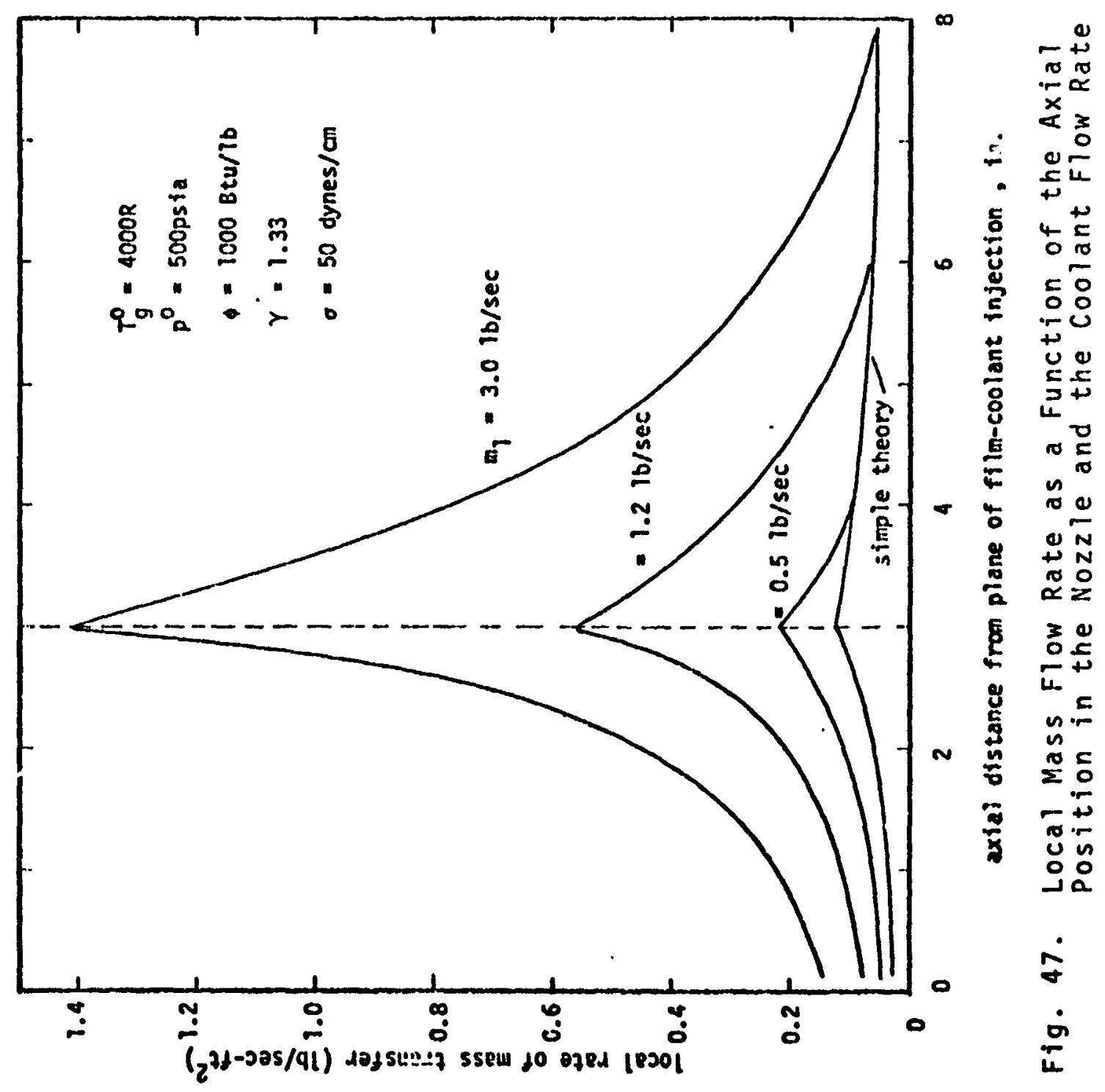


mass transfer occurs at (or very near) the throat of the nozzle. The figure also shows that the local rate of mass transfer predicted by the present theory can be substantially greater than that predicted by the simple theory. Of course, if the liquid coolant has a surface tension less than the 50 dynes/cm that is represented by the calculated results in Fig. 47, then the local rates of mass transfer would be substantially greater than those shown.

In summary, the illustrative example presented in this subsection suggests that the simple theory for mass transfer can lead to a catastrophic design of a liquid-fiim cooled exhaust nozzle. In most cases that are of practical importance, the entrainment phenomenon would completely dominate the mass transfer process.

It also follows from the results of the foregoing calculations that it is not practical, in general, to introduce the film coolant at only one location. The net rate at which liquid is entrained into the gas stream can be reduced substantially if the coolant is introduced at several axial locations. It would seem that this particular problem needs further experimental investigation. 


\section{CONCLUSIONS}

The following principal conclusions are drawn from the results of the subject investigation.

1. The scale of the small disturbance waves on the surface of the liquid film is principally characterized by the momentum parameter $M_{0}$ for the gas stream, with the scale decreasing with increasing values of $M_{0}$.

2. The surface tension apparently affects the film surface characteristics because with water as the liquid phase (relatively high values of $\sigma$ ), a second type of disturbance wave was frequently observed (two dimensional, long wavelength) that was never observed with either methanol, butanol, or RP-l as the liquid phase (relatively low values of surface tension).

3. The basic interfacial structure is not altered appreciably by substantial variations in the liquid viscosity. Also it is not noticeably influenced by a significant variation in the rate of liquid flow in the liquid tilm so long as the gas stream velocity is greater than about 50 fps.

4. The rate of mass transfer from a thin iquid film into a high velocity, hot gas strean can be substantially greater (typically 5 to 10 times greater) than that predicted by the 
simple theory for mass transfer, with the phenomenon of entrainment normally accounting for the bulk of the mass transfer.

5. The mass transfer due to entrainment is a function of the rate of liquid injection $m_{1}$, the momentum parameter $M_{0}$, and the surface tension $\sigma$, with the amount of liquid entrained into the gas stream ilccreasing with increasing values for $m_{1}$ and $M_{0}$ and decreasing values for $\sigma$.

6. The liquid viscosity was not found to influence the rate of entrainment, thereby contradicting the proposal by many observers that the liquid-film Reynolds number is a primary parameter characterizing the phenomenon of entrainment. 7. The "effective" roughness of the liquid film can result in an increase in the evaporative transfer of mass of as much as 80 percent or more over that predicted by the simple theory for mass transfer.

8. The data fur mass transfer with a constant velocity gas stream was satisfactorily correlated in terms of two dimensional parameters, one characterizing the entrainment phenomenon and one characterizing the phenomenon of film surface roughness. The comparison of the experimental data with the present theory showed that the agreement was generally to within 5 to 10 percent.

9. There is some physical evidence that the dimensional entrainment group $x_{e}$ is equivalent to a dimensionless Weber number if the characteristic length for. the latter 
is taken as the scale of the small disturbance waves on the surface of the liquid film. Moreover, there is some evidence that the phenomenon of entrainment of liquid from the surface of a thin liquid film is analogous to the phenomenon of atomization of liquid drops suspended in a gas stream. 10. The data for mass transfer with an accelerating gas stream was satisfactorily correlated by locally applying the correlation that was developed for the mass transfer data with a constant velocity gas stream and then numerically integrating over the length of the liquid film to obtain the total mass transfer.

11. The analysis that was developed to extend the results of the present investigation to the general case of an arbitrary film cooled length was shown to agree favorably with the experimental data of Refs. (19) and (20). This favorable comparison also suggests that the relatively simple mass transfer correlation developed herein has considerable utility because the flow parameters that characterized these two investigations were substantially different from those that characterized the subject investigation.

12. The liquid coolant requirements for cooling the nozzle of a high energy propulsion device can be reduced substantially by injecting the liquid coolant at several axial locations, and by selecting coolants which have a high surface tension. 
13. Correlation of the data for the maximum liquid temperature showed that the simple theory developed herein predicted that temperature with sufficient accuracy for most engineering-type calculations. 


\section{BIBLIOGFAPHY}

1. Coulbert, C. D., "Selecting Cooling Techniques for Liquid Riscets for Spacecraft," Journal Spacecraft and Rockets, March-April 1964.

2. Kramer, E. L., and Gronich, S., "A Transpiration-Cooled Nozzle a: Applied to a Gas-Core Nuclear Propulsion System," Journal Spacecraft and Rockets, May-June 1965.

3. Benser, W. A., and Graham, R. W., "Hydrogen Convective Cooling of Rocket Nozzles," ASME Paper 62-AV-22 (1962).

4. Kelley, J.B., and L'Ecuyer, M. R., "Tianspiration cooling, its theory and application," Jet Propulsion Center, Purdue University, Report No. TM-66-5, June 1966 .

5. Charvonia, D. A., "A study of the mean thickness of the liquid film and the characteristics of the interfacial surface in annular, two-phase flow in a vertical pipe," PhD Thesis, Purdue University, Lafayette, Indiana, May 1959 .

6. Schneiter, G. R., "ilass transfer in annular two-phase flow," Pho Thesis, Purdue University, Lafayette, Indiana, June 1966 .

7. Craik, A. D. D., "Wind-generated waves in thin liquid films," J. Fluid Mech., 26, 369, Part 2, 1966.

8. Knuth, E. L., "The mechanics of film cooling-Part 1," Jet Propulsion, Nov.-Dec. 1954, p. 359.

9. Kinney, G. R., and Abramson, A. E., "Investigation of Annular Liquid Flow with Cocurrent Air Flow in Horizontal Tubes," NACA RM E5 C 3 (1951).

10. Tate, K., "Liquid-film cooling, a cold flow study of film instability and entrainment," MSME Thesis, Purdue University, Lafayette, Indiana, June 1967. 
11. Levy, S., "Prediction of two-phase annular flow with liquid entrainment," Int. J. Heat Mass Transfer, $\underline{9}$, 171, (1966).

12. Collier, J. G., and Hewitt, G. F., Trans. Inst. Chem. Engrs., 39, 127 (1961).

13. Murgatroyd, W., "The role of shear and form forces in the stability of a dry patch in two-phase film flow," Int. J. Heat Mass Transfer, 8, 297, (1965).

14. Hartley, D. E., and Murgatroyd, W., "Criteria for the bread-up of thin liquid layers flowing isothermally over solid surfaces," Int. J. Heat Mass Transfer, $\underline{7}$, 1003 , (1964).

15. Hewitt, G. F., and Lacey, P. M. C., "The breakdown of the liquid film in annular two-phase flow," Int. J. Heat Mass Transfer, $8,781,(1965)$.

16. Wicks, M., and Duker, "Entrainment and Pressure Drop in Co-current Gas-Liquid Flow, Part 1," AIChE Journal, 6, 463-468, (1960).

17. Martinelli, R. C., Boelter, L. M. K., Taylor, T. H. M., Thomsen, E. G., and Morrin, E. H., "Isothermal Pressure Drop for Two-Phase Two-Component Flow in a Horizontal Pipe," Trans. ASME, 66, 139, (1944).

18. Collier, J. G., and Wallis, G. B., "Two Phase Flow and Heat Transfer," 4 Volumes, Notes for a Summer Course, July 24 - August 4, 1967, Department of Mechanical Engineering, Stanford University, Stanford, California.

19. Kinney, G. R., Abramson, A. E., and Sloop, J. L., "Internal-Liquid-Film-Cooling Experiments with AirStream Temperatures to $2000^{\circ} \mathrm{F}$ in 2- and 4-Inch Diameter Horizontal Tubes," NACA Report 1087 (1952).

20. Emmons, D. L., "Effects of Selected Gas Stream Flow Parameters and Coolant Physical Properties on Film Cooling of Rocket Motors," PhD Thesis, Purdue University, Lafayette, Indiana, August 1962.

21. Knuth, E. L., "The Mechanics of Film Cooling--Part 2," Jet Propulsiull, January 1955.

22. Graham, A. R., "Film Cooling of Rocket Motors, Part II. Film Cooling, Its Theory and Application," PhD Thesis, Jet Propulsion Center, Purdue University, Lafayette, Indiana, January 1958. 
23. Sellers, J.P., "Experimental and Theoretical Study of the Application of Film-Cooling to a Cylindrical Rocket Thrust Chamber," PhD Thesis, Purdue University, Lafayette, Indiana, June 1958.

24. Hermann, R., Leitinger, H., and Melnik, H. L., "Research on the Design of Hypersonic Nozzles at High Stagnation Temperatures-Part 1," WADC TN 55-507, Rosemount Aeronautical Laboratories, Research Report No. 127, March 1955.

25. Gater, R. A,, "Liquid-film cooling, its physical nature and theoretical analysis," MSME Thesis, Purdue University, Lafayette, Indiana, January 1966.

26. Gater, R. A., L'Ecuyer, M. R., Warner, C. F., "Liquidfilm cooling, its physical nature and theoretical analysis," Jet Propulsion Center, Purdue University, Report No. TM-65-6, October 1965.

27. Luikov, A. V., "Heat and Mass Transfer in Evaporation," International Chemical Engineering, April 1963.

28. Crabtree, D. L., "An experimental investigation of the velocity and temperature profiles in the boundary layer above an linstable evaporating film of liquid under the influence of a moving stream of hot gas," PhD Thesis, Purdue University, Lafayette, Indiana, January 1967.

29. Graham, A. R., and Zucrow, M. J., "Some Considerations of Film Cooling for Rocket Motors," Jet Propulsion, June 1957.

30. Graham, A. R., "Film Cooling of Rocket Motors, Part I. An Experimental and Theoretical Investigation of Film Cooling of Rocket Motors," PhD Thesis, Jet Propulsion Center, Purdue University, Lafayette, Indiana, January 1958.

31. Hall Taylor, N., Hewitt, G. F., and Lacey, P. M. C., "The Motion and Frequency of Large Disturbance Waves in Annular Two-Phase Flow of Air-Water Mixtures," Chenical Engineering Science, 18, 537-552, (1963).

32. Chien, S., and Ibele, M., "Pressure Drop and Liquid Film Thickness of Two-Phase Annular and Annular-Mist Flows," Trans. ASME, Ser. C.; J. Heat Transfer 86, $88-96,(1964)$. 
33. Cohen, L. S., and Hanratty, T. J., "Generation of Waves in the Concurrent Flow of Air and a Liquid," AIChE Journa1, 11, 138, (1965).

34. Craik, A. D. D., "Wind Generated Waves in Liquid Films," PhD Dissertation, University of Cambridge, 1965.

35. Hanratty, T. J., and Engen, J. M., "Interaction Between a Turbulent Air Stream and a Moving Water Surface," AIChE Journal, September 1957.

36. Kays, W. M., Convective Heat and Mass Transfer, McGrawHill, Inc., New York, 1966.

37. Steen, D. A., and Wallis, G. B., "The Transition from Annular to Annular-Mist, Co-Current Two-Phase Downflow," Report, No NYO-3114-2, Dartmouth College, 1964.

38. Steen, D. A., MS Thesis, Dartmouth College, 1964.

39. Hinze, J. 0., Turbulenca, McGraw-Hill, Inc., New York, 1959 .

40. Eckert, E. R. G., and Drake, R. M., Jr., Heat and Mass Transfer, McGraw-Hi11, Inc., New York, 1959.

41. Keenan, J. H., and Keyes, F. G., Thermodynamic Properties of Steam, First Edition, John Wiley and Sons, Inc., New York, 1936.

42. Rannie, W. D., "A Simplified Theory of Porous Wall Cooling," Progress Report No. 4-50 Jet Propulsion Laboratory, California Institute of Technology, Pasadena, California, November 1947.

43. Rannie, J. B., "The Influence of Selected.Gas Stream Parameters on the Film Cooling Requirements of Rocket Motor Combustion Chambers, "MSME Thesis, Purdue University, Lafayette, Indiana, 1962.

44. Humble, L. V., Lowdermilk, W. H., and Grele, M. D., "Hieat Transfer Coefficients and Friction Factors for Air Flowing in a Tube at High Surface Temperatures," ASME Heat Transfer and Fluid Mechanics Institute, 1949 , pp. 165-180.

45. Guinn, G. R., "The Influence of Dual Injection Slots on the Film Cooling of Rocket Motor Combustion Chambers," MSME Thesis, Purdue University, Lafayette, Indiana, January 1963. 
46. Hatch, J. E., and Papell, S. S., "Use of a Theoretical Flow Model to Correlate Data for Film Cooling or Heating an Adiabatic Wall by Tangential Injection of Gases of Different Fluid Properties," NASA TN D-130, November 1959.

47. Persh, J., and Lee, R., "A Method for Calculating Turbulent Boundary Layer Development in Supersonic and Hypersonic Nozzles Including the Effects of Heat Transfer," U. S. Naval Ordnarice Lab. Report 4200, 1956.

48. Schlichting, H., Boundary Layer Theory, Fourth Edition, McGraw-Hill, Inc., New York, 1960.

49. Bartz, D. R., "An Approximate Solution of Compressible Turbulent Boundary-Layer Development and Convective Heat Transfer in Convergent-Divergent Nozzles," ASME Trans., November 1955.

50. Elliot, D. G., Bartz, D. R., and Silver, S., "Calculation of Turbulent Boundary-Layer Growth and Heat Transfer in Axi-Symmetric Nozzles," Jet Propulsion Lab. TR 32-387, February 1963.

51. Librizzi and Cresci, R. J., "Transpiration Cooling of a Turbulent Boundary Layer in an Axisymmetric Nozzle," AIAA Journal, April 1964.

52. Libby, P. A., and Cresci, R. J., "Experimental Investigation of the Downstream Influences of Stagnation Point Mass Transfer," Journal Acrospace Sciences, January 1961.

53. Seban, R. A., "Heat Transfer and Effectiveness for a Turbulent Boundary Layer with Tangential Fluid Injection," ASME Paper No. 59-A-177 (1960).

54. Seban, R. A., and Back, L. H., "Effectiveness and Heat Transfer for a Turbulent Boundary Layer with Tangential Injection and Variable Free-Stream Velocity," Journal Heat Transfer, August 1962.

55. Hartnett, J. P., Birkebak, R. C., and Eckert, E. R. G., "Velocity Distributions, Temperature Distributions. Effectiveness and Haat Transfer for Air Ir: $:=?: 2 d$ Through a Tangential slot Into a Turbulent Boundary Layer," Journal Heat Transfer, August 1961.

56. McAdams, W. H. Heat Transmission, McGraw-Hill, Inc., New York, 1954. 
57. Maxwell, J. B., Data Book on Hydrocarbons, D. Van Nostrand Co., 1950 .

58. Handbook of Chemistry and Physics, 4lst Edition, 1959-1960, Chemical Rubber Publishing Co.

59. Reid, R. C., and Sherwood, T. K., The Properties of Gases and Liquids, McGraw-Hili, Inc., New York, 1958 .

60. Liquid Propellants Handbook, Battelle Memorial Institute.

61. Freon, Technical Literature File for Contractors and Dealers, Freon Products Division, E. I. Du Pont de Nemours \& Co. (Inc.). 
NOTATION

\begin{tabular}{|c|c|}
\hline $\mathbf{a}$ & acoustic speed (Eq. $(3-69))$ \\
\hline A & constant (see Eq. $(3-1))$ \\
\hline b & constant (Eq. $(3-63))$ \\
\hline B & constant (see Eq. $(3-2))$ \\
\hline$B_{h}$ & heat-mass transfer parameter (Eq. (3-22)) \\
\hline C & mass concentration of vapor \\
\hline$c_{f}$ & skin friction coefficient (Eq. $(3-20)$ ) \\
\hline$c_{p}$ & specific heat at constant pressure \\
\hline$c_{v}$ & specific heat at constant volume \\
\hline d & constant (Eq. $(3-73))$ \\
\hline D & tube diameter \\
\hline $\mathbf{e}_{\mathbf{0}}$ & entrainment parameter (see Eq. $(3-5)$ ) \\
\hline f & denotes a function \\
\hline $\mathbf{F}$ & correction factor (see Eq. $(3-16)$ ) \\
\hline $\mathbf{G}$ & mass velocity for gas stream (Eq. $(2-1)$ ) \\
\hline $\mathbf{h}$ & specific enthalpy \\
\hline h & heat transfer coefficient (Eq. $(1-2)$ ) \\
\hline$H_{v}$ & latent heat of vaporization \\
\hline$k$ & thermal conductivity \\
\hline K & dimensional constant (Eq. $(3-41))$ \\
\hline
\end{tabular}




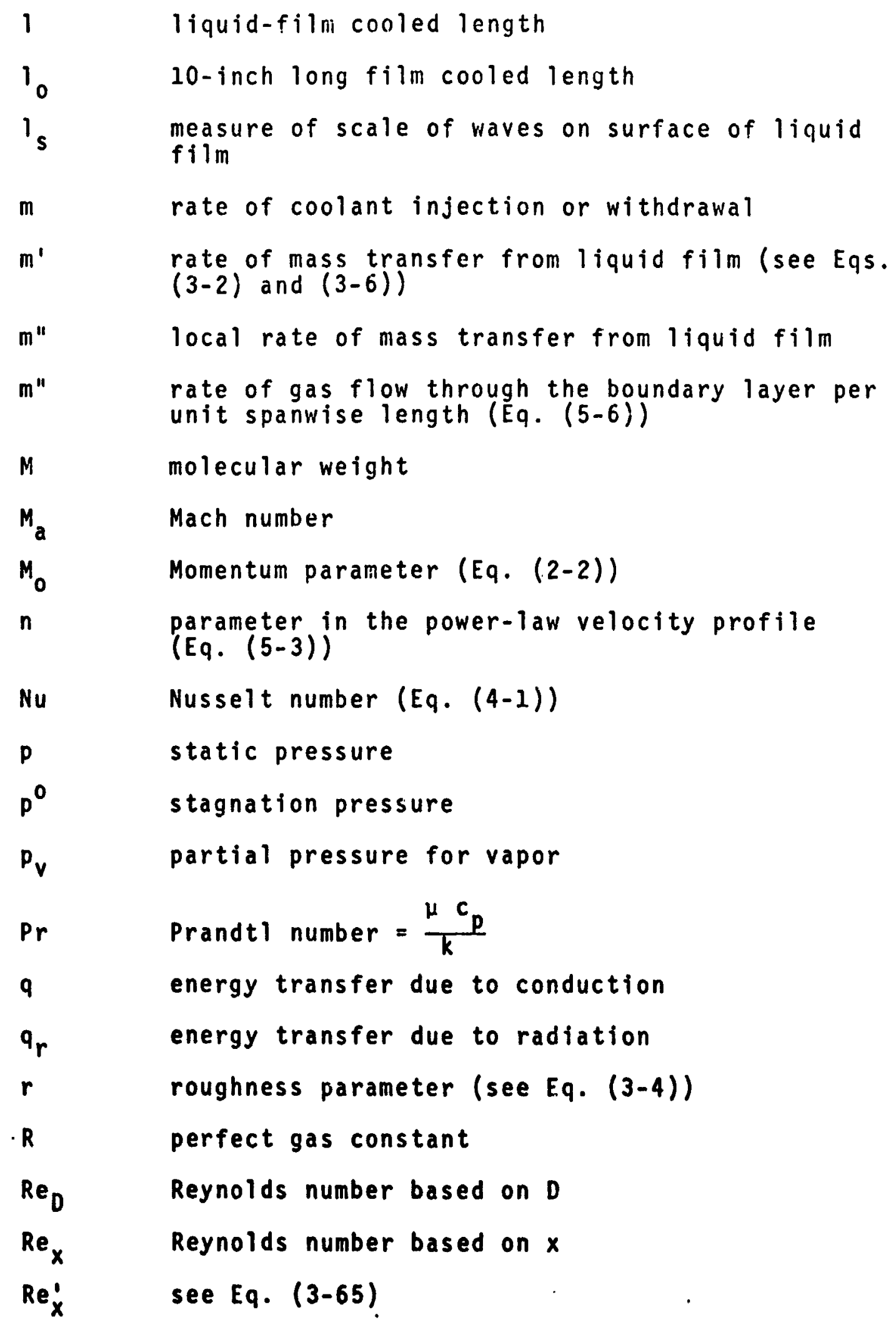




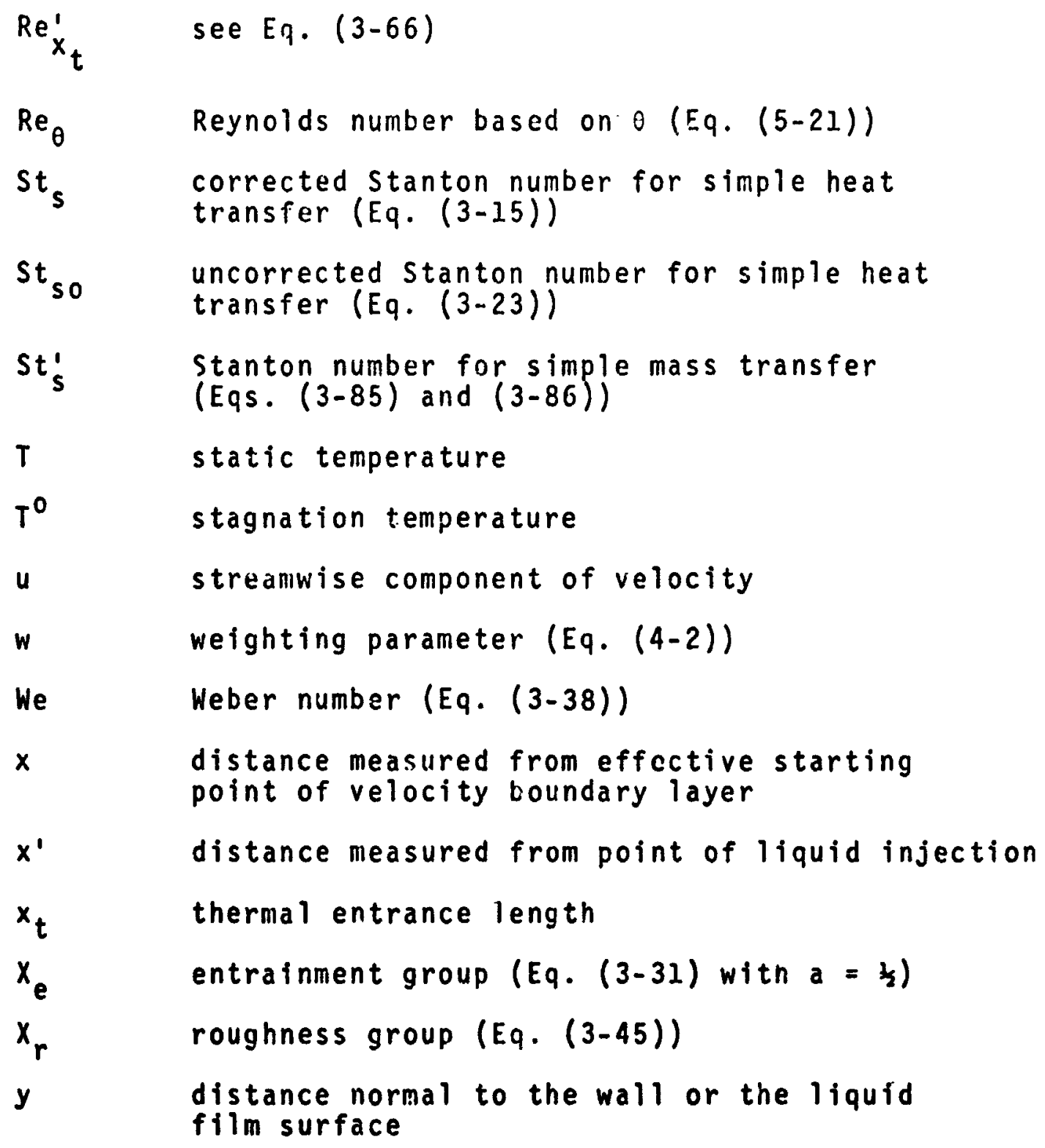

\section{Greek}

r

$\delta$

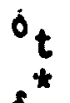

$\mu$

specific heat ratio

dynamic boundary layer thickness

thermal boundary layer thickness

displacement thickness (Eq. (5-14))

momentum thickness (Eq. $(5-15)$ )

dynamic viscosity 


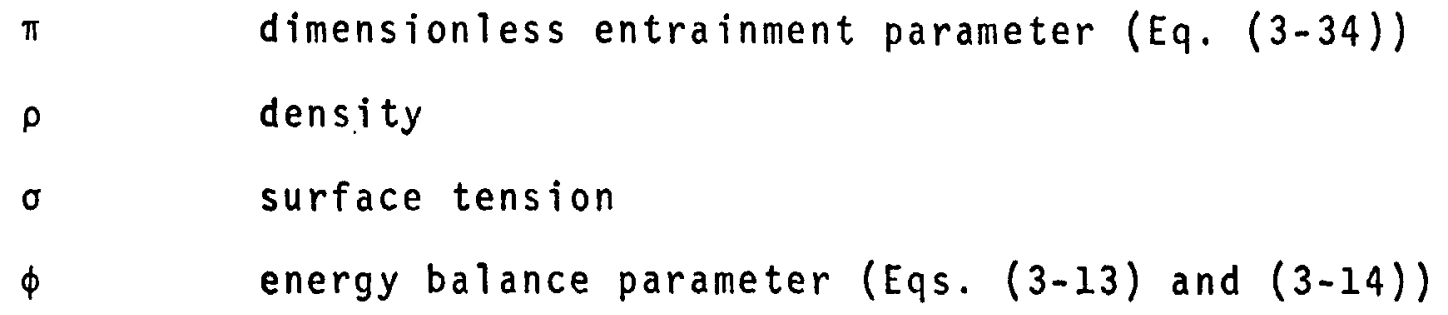

\section{Subscripts}

1

2 evaluated at the terminus of the liquid-film cooled length

3

evaluated at the terminus of the gas-vapor cooled length

aw

adiabatic wall

c

critical

e

effective

g

main-stream state $(y=\delta)$

g

main-stream gas

gv

gas-vapor cooling

k

summation index

1

liquid

L

evaluated at the gas-liquid interface but in the liquid phase

m

maximum

$\mathbf{r}$

reference

S

evaluated at the gas-liquid interface

s

simple

S

evaluated at the gas-liquid interface but in the gas phase

v

vapor 
216

w

wal 1

Superscripts

0

stagnation conditions

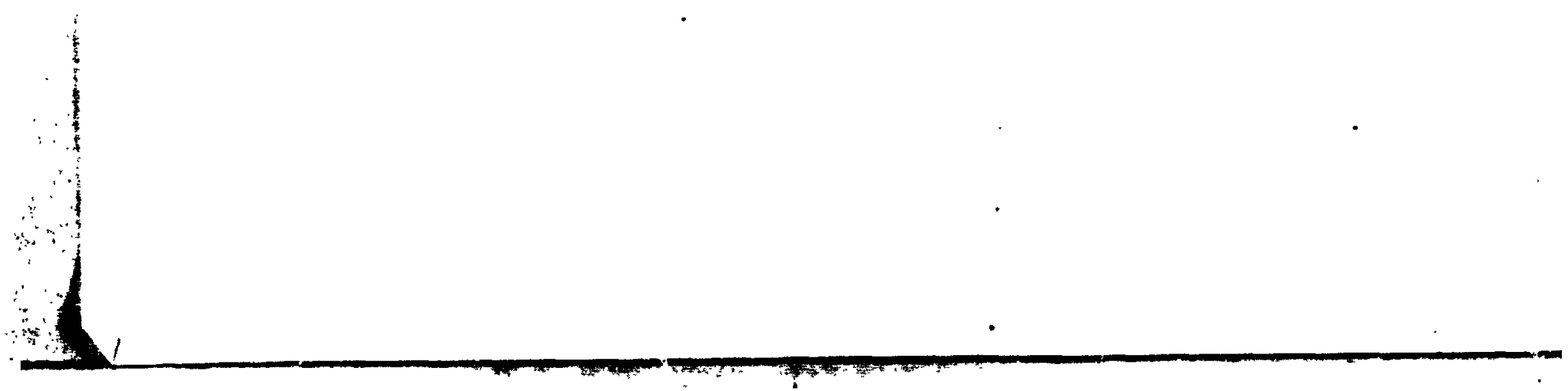


APPENDIX A

AN ANALYSIS FOR THE GAS-VAPOR-COOLED REGION

\section{A.1 Introduction}

It is difficult, if not impossible, to indic a cumplete mathematical analysis of the boundary layer and heat transfer characteristics of the region immediately downstream from the end of the liquid film. Moreover, an involved analysis would not be generally warranted because of the large number of assumptions that must be made concerning the velocity and temperature profiles, the form factor or factors, the skin-friction law, the heat-transfer law, etc. As a consequence of this fact, the analysis presented herein is based on an idealized and relatively simple flow model. It will be shown that the analysis cannot be completely developed until more definitive experimental data are obtained for the subject problem. However, the limited data that do exist show that the analysis presented herein has considerable promise. These data are discussed in subsection A.2.4.

Both Emmons (20) and Guinn (45) developed semiempirical analyses for the gas-vapor-cooled region; these 
analyses were based on the flow model suggested by Hatch and Papell (46) for gas-film cooling. The analysis presented here differs from those due to Emmons and to Guinn in the following fundamental respects:

(a) boundary-layer type flow rather than fully-developed pipe low is considered;

(b) the analysis is generalized so that it can be applied to a compressible gas flow in the presence of a streanwise pressure gradient; and

(c) no direct similarity between gas-vapor and gas-film cooling is assumed.

As discussed in subsection 2.1.2, an analysis for the gas-vapor-cooled region requires the consideration of two cases: (a) the case where the wall is adiabatic; and (b) the case where the wall is externally cooled. These two cases are considered in Sections A.2 and A.3, respectively.

\section{A.2 The Case Where the Wall is Adiabatic}

\section{A.2.1 The Flow Model}

The flow model employed for the analys is of the gasvapor-cooled region is defined by the following conditions.

(1) The wall is adiabatic.

(2) The boundary layer flow is fully turbulent.

(3) The thermal, the diffusional, and the dynamic boundary layer thicknesses are equal; the latter is denoted by $y=\delta$. 
(4) The stagnation temperature for the main stream, T, is a constant in the streamwise direction.

(5) The specific heat for the gas, $c_{p}$, is a constant.

(6) Radiation heat transfer and chemical reactions within the boundary layer are not important.

(7) The velocity profile and the stagnation-temperature profile for the boundary layer are of the function forms:

$$
\frac{u}{u_{g}}=f_{1}\left(\frac{y}{\delta}\right)
$$

and

$$
\frac{T^{0}-T_{a w}}{T_{g}^{0}-T_{a w}}=f_{2}\left(\frac{y}{\delta}\right)
$$

where $T_{a w}$ is the temperature of the fluid adjacent to the adiabatic wall. To illustrate, $f_{1}$ could be assumed of the power-law form:

$$
f_{1}=\left(\frac{y}{\delta}\right)^{1 / n}
$$

and $f_{2}$ could be assumed of the form

$$
f_{2}=A+B\left(\frac{y}{\delta}\right)+C\left(\frac{y}{\delta}\right)^{2}+D\left(\frac{y}{\delta}\right)^{3}
$$

The constants in Eq. (A-4) can be evaluated from the 
following boundary conditions that apply to $f_{2}$. Thus

$$
\begin{aligned}
& f_{2}(0)=0 \\
& f_{2}(1)=1 \\
& \frac{\partial f_{2}}{\partial y}(0)=0 \\
& \frac{\partial f_{2}}{\partial y}(1)=0
\end{aligned}
$$

The resultant expression for $f_{2}$ is

$$
f_{2}=3\left(\frac{y}{\delta}\right)^{2}-2\left(\frac{y}{\delta}\right)^{3}
$$

For the present analysis, however, the actual forms of $f_{1}$ and $f_{2}$ need not be assumed, only the assumption that $f_{1}$ and $f_{2}$ exist for the gas-vapor-cooled region is required.

\section{A.2.2 The Energy Balance}

Figure A.l presents, schematically, a control volume for the gas-vapor-cooled region; the control surface of the control volume is defined by $x=x_{2}, x=x, y=\delta$, and $y=0$. The following energy fluxes, written in accordance with the flow model defined in the foregoing section, are presented in the figure:

\footnotetext{
(a) $\int_{0}^{\delta_{2}} \rho u c_{p} T^{0} d y=$ the rate at which thermal and $k i-$ neicic eneigy is transferred into the control volume per unit spanwise length at the station $x=x_{2}$.
} 
I

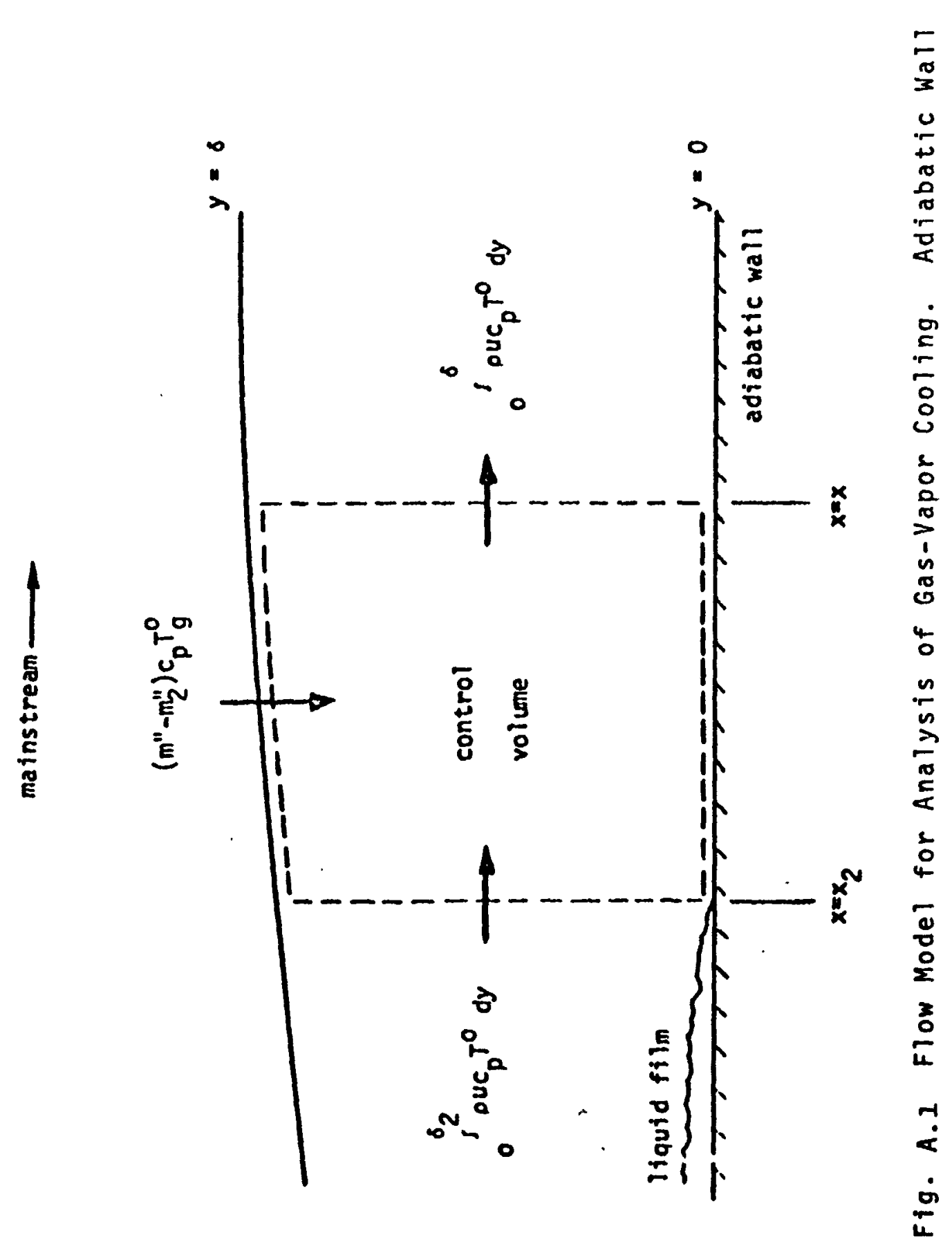


(b) $\begin{aligned} \int_{0}^{\delta} \rho u c_{p} T^{0} d y= & \text { the rate at which thermal and } k i- \\ & \text { netic energy is transferred out of } \\ & \text { the control volume per unit span- } \\ & \text { wise length at the station } x=x .\end{aligned}$

(c) $\left(m^{\prime \prime}-m_{2}^{\prime \prime}\right) c_{p} T_{g}^{0}=$ the rate at which thermal and $k i-$ netic energy is transferred from the main stream into the control volume per unit spanwise length.

The parameter $m "$ in term (c) is defined as follows:

$$
\begin{aligned}
m^{\prime \prime}=\int_{0}^{\delta} \rho u d y= & \text { the rate at which mass is trans- } \\
& \text { ferred through the boundary layer } \\
& \text { per unit spanwise length at the } \\
& \text { station } x=x
\end{aligned}
$$

The energy balance for the control volume in Fig. A.l can be written as follows. Thus

$$
\int_{0}^{\delta_{2}} \rho u c_{p} T^{0} d y+\left(m^{\prime \prime}-m_{2}^{\prime \prime}\right) c_{p} T_{g}^{0}=\int_{0}^{\delta} \rho u c_{p} T^{0} d y
$$


Employing Eq. $(A-6)$, and dividing through by $c_{p}, E q .(A-7)$ can be written in the form

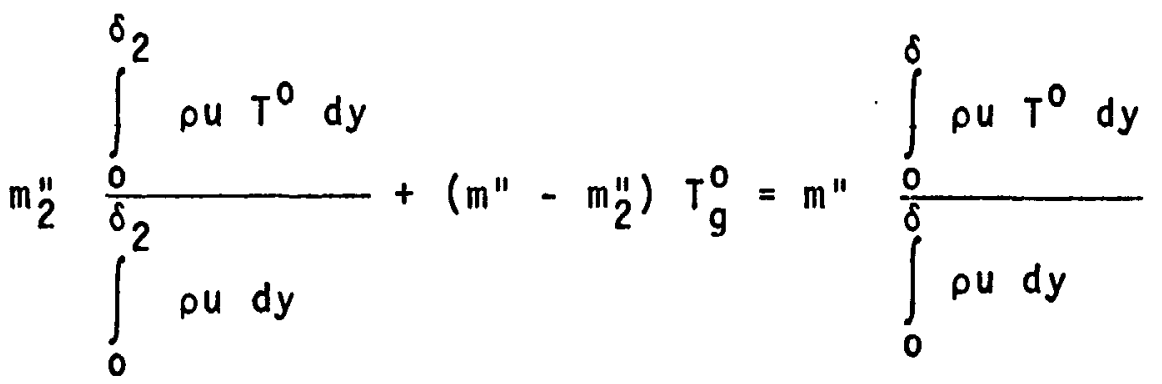

$(A-10)$

In order to realize the desired simplicity of the present analysis, the following approximation is introduced concerning the integral terms in Eq. (A-10). Thus*

$$
\frac{\int_{0}^{\delta} \rho u T^{0} d y}{\int_{0}^{\delta} \rho u d y} \cong \frac{\int_{0}^{\delta} u T^{0} d y}{\int_{0}^{\delta} u d y}
$$

Thus, Eq. $(A-10)$ can be written in the following form by introducing the above approximation and assumption (7) of the flow model:

* Note that this approximation is not equivalent to and is less restrictive than the assumption of constant density. 


$$
\begin{aligned}
& m_{2}^{\prime \prime} \frac{\int_{0}^{\delta_{2}} f_{1}\left\{\left(T_{g}^{0}-T_{a w, 2}\right) f_{2}+T_{a w, 2}\right\} d y}{\int_{0}^{\delta_{2}} f_{1} d y}+\left(m^{\prime \prime}-m_{2}^{\prime \prime}\right) T_{g}^{0}= \\
& m^{\prime \prime} \frac{\int_{0}^{\delta} f_{1}\left\{\left(T_{g}^{0}-T_{a w}\right) f_{2}+T_{a w}\right\} d y}{\int_{0}^{\delta} f_{1} d y}
\end{aligned}
$$

The latter expression can be rewritten in the form

$$
\begin{gathered}
m_{2}^{\prime \prime}\left(T_{g}^{0}-T_{a w, 2}\right)-m_{2}^{\prime \prime}\left(T_{g}^{0}-T_{a w, 2}\right) \frac{\int_{0}^{1} f_{1} f_{2} d\left(\frac{y}{\delta}\right)}{\int_{0}^{1} f_{1} d\left(\frac{y}{\delta}\right)}= \\
m^{\prime \prime}\left(T_{g}^{0}-T_{a w}\right)-m^{\prime \prime}\left(T_{g}^{0}-T_{a w}\right) \frac{\int_{0}^{1} f_{1} f_{2} d\left(\frac{y}{\delta}\right)}{\int_{0}^{1} f_{1} d\left(\frac{y}{\delta}\right)} .
\end{gathered}
$$

Finally, the terms in Eq. (A-11) can be combis:: iu yield the following energy balance for the control volume presented in Fig. A.1. Thus 


$$
\frac{T_{g}^{0}-T_{a w}}{T_{g}^{0}-T_{a w, 2}}=\frac{m_{2}^{\prime \prime}}{m^{\prime \prime}}
$$

The relatively simple form of $\mathrm{Eq} .(A-12)$ is due principally to assumption (7) of the flow model.

Equation $(A-12)$ enables the determination of the adiabatic-wall temperature $T_{a w}$ as a function of $x$ once $T_{a w, 2}$ and $T_{g}^{0}$ are specified and $m^{\prime \prime}$ as a function of $x$ is known. The parameter $T_{g}^{0}$ is normally known from the statement of the problem, and the parameter $T_{a w, 2} c a n$ be estimated from the derelopments presented in subsection 3.4.1. The evaluation of $\mathrm{m}^{\prime \prime}$ as a function of $x$ is considered in subsection A.2.3.

\section{A.2.3 The Evaluation of $\mathrm{m}^{\prime \prime}$}

Equation (A-6), which defines the parameter $m$ ", can be rewritten in the form

$$
m^{\prime \prime}=(\rho u)_{g}\left(\frac{\delta}{\theta}-\frac{\delta^{\star}}{\theta}\right) \theta
$$

where

$$
\delta^{*}=\int_{0}^{\delta}\left(1-\frac{\rho u}{(\rho u)_{g}}\right) d y=\text { the displacement thickness }
$$


$\theta=\int_{0}^{\delta} i \frac{\partial u}{\partial u)_{g}}\left(1-\frac{u}{u_{g}}\right) d y=$ the momentum thickness

Equation $(A-13)$ shows that $m "$ can be determined as a function of $x$ if $\frac{\delta}{\theta}, \frac{\delta^{*}}{\theta}$, and $\delta$ are known as functions of $x$. The evaluation of these quantities is considered in the following paragraphs.

The Evaluation of $\delta / \theta$ and $\delta * / \theta$. Persh and Lee (47) have tabulated the values of $\delta / \theta$ and $\delta * / \theta$ as funitions of the main-stream Mach number and the wall-to-main-stream temperature ratio. It is suggested, therefore, that these tabulated data be employed for evaluating $\delta / \theta$ and $\delta * / \theta$ when the variation in the density for the gas stream across the boundary layer is significant. However, for the more general case where that density variation is not significant, it is suggested thit the subject parameters be calculated by applying the power-law velocity profile (see Eq. (A-5)). The resultant expressions for $\delta / \theta$ and $\delta * / \theta$ are $(48, p, 536)$

$$
\frac{\delta}{\theta}=\frac{(1+n)(2+n)}{n}
$$

and

$$
\frac{\delta^{*}}{\theta}=\frac{(2+n)}{n}
$$


The Evaluation of $\theta$. To determine the value of the momentum thickness $\theta$ as a function of $x$, one must solve the momentum integral equation. That equation can be written in the form (49)

$$
\frac{c_{f}}{2}=\frac{d \theta}{d x}+\theta\left[\left(2+\frac{\delta}{\theta}-\right) \frac{I}{u_{g}} \frac{d u_{g}}{d x}+\frac{1}{\rho_{g}} \frac{d \rho_{g}}{d x}\right]
$$

Or in the form $(50)$

$$
\frac{c_{f}}{2}=\frac{d \theta}{d x}+\theta\left[\frac{1}{M_{a}} \frac{d M_{a}}{d x} \frac{\left\{\left(\delta^{*} / \theta\right)+2-M_{a}^{2}\right\}}{\left\{1+\left(\frac{y-I}{2}\right) M_{a}^{2}\right\}}\right]
$$

where

$$
\frac{c_{f}}{2}=\frac{\tau_{w}}{\rho_{g} u_{g}^{2}}=\text { the skin-friction coefficient }
$$

To solve* either Eq. $(A-18)$ or Eq. $(A-19)$ for $\theta$, one must specify the form of the skin-friction law; the latter is denoted functionaliy by

$$
\frac{c_{f}}{2}=\frac{c_{f}}{2}\left(\operatorname{Re}_{\theta}\right)
$$

*A numerical solution is generally required for either Eq. $(A-18)$ or $(A-19)$. 
where

$$
\begin{aligned}
\operatorname{Re}_{\theta}=\frac{\rho_{g} u_{g} \theta}{\mu_{g}}= & \text { the Reynolds number for the main } \\
& \text { stream based on the characteristic } \\
& \text { length } \theta
\end{aligned}
$$

Several empirical skin-friction laws are presented in Chapter 22 of Ref. (48) for the case where the gas flow is turbulent, incompressible, and over a flat plate. In the analysis of gas flows with favorable pressure gradients, it is normally assumed that the skin-friction law is the same as that established empirically for the fiatplate case $(49,50,48)$. Furthermore, the case where heat transfer effects and/or compressibility effects are important can be analyzed by evaluating the thermodynamic properties for the gas stream at an appropriate reference temperature. For example, the reference temperature, denoted by $T_{r}$, can be determined from (36):

$$
T_{r}=0.5\left(T_{g}+T_{w}\right)+(P r)^{1 / 3} \frac{u_{g}^{2}}{2 c_{p g}}
$$

It is suggested that in the evaluation of the physical properties that the composition of the gas be assumed uniform throughout the boundary layer and equal to that for the main stream; i.e., assume that a reference temperature is sufficient to define the reference siate.

To complete the solution for $\theta$, the boundary condition 
on $\theta$ at the station $x=x_{2}$, denoted by $\theta_{2}$, must be specified. An approximate technique for the evaluation of that boundary condition was proposed in Ref. (25). However, subsequent analysis of the available experimental data suggests that that proposal is not satisfactory. In order to illustrate that point, an analysis of the data that are presented in Ref. (19) is discussed in Subsection A.2.5. However, to facilitate that discussion, it is convenient to first consider a special case for which the foregoing developments are simplified substantially.

\section{A.2.4 A Special Case}

Consider the special case that is defined by the following conditions.

1. The gas flow is incompressible, constant density, and over an adiabatic flat plate.

2. The skin-friction law is that due to Prandtl (48, Eq. 22.7). Thus

$$
\frac{c_{f}}{2}=0.0128 \operatorname{Re}_{\theta}^{-\frac{3}{4}}
$$

3. The velocity profile for the boundary layer is given by Eq. $(A-3)$. Thus

$$
\frac{u}{u_{g}}=\left(\frac{y}{\delta}\right)^{1 / n}
$$


For this special case, Eq. (A-12) reduces to the form

$$
\frac{T_{g}-T_{a w}}{T_{g}-T_{a w, 2}}=\frac{m_{2}^{\prime \prime}}{m^{\prime \prime}}
$$

Equation $(A-13)$ reduces to (refer to Eqs. $(A-16)$ and $(A-17)$ )

$$
m^{\prime \prime}=(\cdot-n) \rho u_{g} \theta
$$

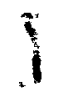

Equation (A-18) simplifies to the form

$$
\frac{c_{f}}{2}=\frac{d \theta}{d x}=\frac{d \operatorname{Re}_{\theta}}{d \operatorname{Re}_{x}}
$$

where

$$
\begin{aligned}
& \operatorname{Re}_{x}=\frac{\rho u_{g} x}{\mu}=\text { the Reynolds number for the main } \\
& \text { stream based on the characteristic } \\
& \text { length } x
\end{aligned}
$$

Note that due to assumption (1), the distance $x$ is measured from the "effective" starting point for the growth of the turbulent boundary layer over the gas-vapor cooled region. It follows from the discussion presented below that the specification of that starting point is equivalent to the specification of the parameter $\theta_{2}$. 
Upon combining Eqs. $(A-23)$ and $(A-24)$, one obtains

$$
\frac{T_{g}-T_{a w}}{T_{g}-T_{a w, 2}}=\frac{{ }_{2}}{\theta}
$$

In addition, substitution of Eq. (A-22) into Eq. (A-25)

yields

$$
0.0128 \mathrm{dRe}_{x}=\operatorname{Re}_{\theta}^{\frac{3}{4}} d \operatorname{Re}_{\theta}
$$

Integrating Eq. $(A-28)$ and cubstituting the limit $\operatorname{Re}_{\theta}=\operatorname{Re}_{\theta_{2}}$ at $x=x_{2}$, one can readily show that

$$
\frac{\operatorname{Re}_{\theta_{2}}}{\operatorname{Re}_{\theta}}=\frac{{ }_{2}}{\theta}=\left(\frac{x_{2}}{x}\right)^{4 / 5}
$$

Substituting Eq. $(A-29)$ into Eq. $(A-27)$ results in

$$
\frac{T_{g}-T_{a w}}{T_{g}-T_{a w, 2}}=\left(\frac{x_{2}}{x}\right)^{4 / 5}
$$

Equation (A-30) presents, for the subject special case, a simple expression for the calculation of the adiabatic-wall temperature $T_{a w}$ as a function of the distance from the end of the liquid film $\left(x-x_{2}\right)$. The problem, therefore, is to determine $x_{2}$. Tha $13 \pm s$ of Ref. (19) give some insight into the problem of the evaluation of that parameter. Those data are considered in the following subsection. 


\section{A.2.5 Experimental Data for the Adiabatic-Wall Temperature From Reference (19)}

Kinney, Abramson, and Sloop (19) present some data for the temperature for an adiabatic wall immediately downstream of a liquid-film cooled wall region*. To the author's knowledge, these are the only such data that appear in the published literature. The experimental apparatus that was employed in the subject investigation was discussed previously in subsection 4.3.2. The reported data were obtained with the 4-inch diameter smooth surface tube at a nominal gas stream temperature of $1400 \mathrm{~F}$ and a nominal gas stream Reynolds number of $5.3 \times 10^{5}$. The length of the liquid-film cooled region was approximately 19.0 inches.

To obtain an evaluation of the present analysis, it is assumed that Eq. $(A-30)$ that was developed for the aforementioned special case is applicable to the subject data. The determination of the value for $x_{2}$ (and thus the effective starting point for the growth of the boundary layer over the gas-vapor cooled region) for these data gives some insight into the phenomena involved.

Figure A.2 presents a plot of the left-hand side of Eq. $(A-30)$ as a function of $\left(x-x_{2}\right)$, the distance measured from the end of the liquid film. The circles shown in the figure were calculated from the data presented in Fig. 4 of Ref. (19). The solid curves shown in Fig. A.2 were calculated * See Figure 4 of Ref. (19). 


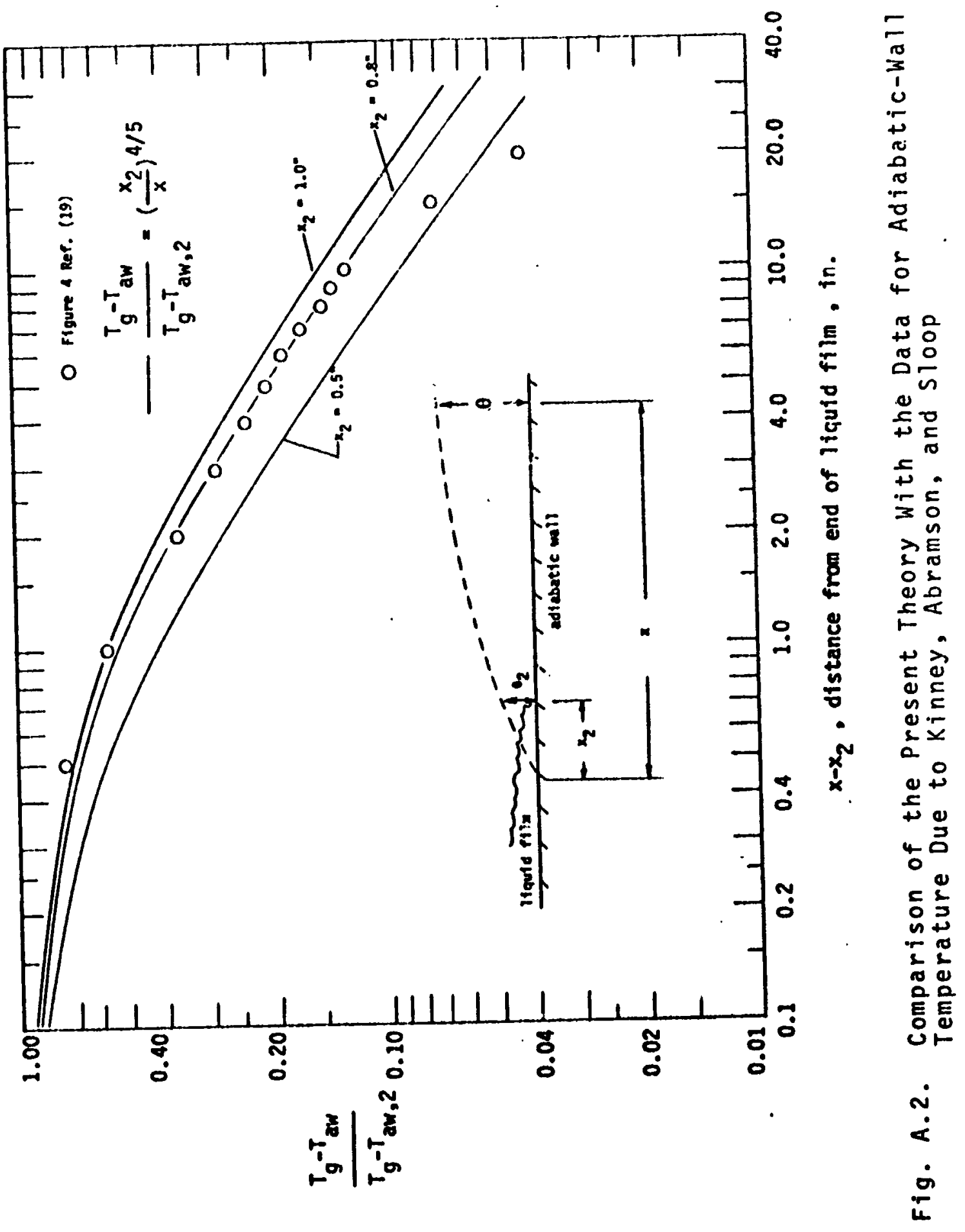


from Eq. $(A-30)$ with values for $x_{2}$ of $0.5,0.8$, and 1.0 inches. For the wall region immediately downstream of the liquid film (say, $x-x_{2}<10.0$ inches), Fig. A.2 shows that a value of 0.8 inches for $x_{2}$ results in an excellent correlation of the experimental data. The small value of 0.8 inches for $x_{2}$, however, is difficult to interpret physically. This small value suggests that the boundary layer phenomena are complex, with perhaps a considerable change in the boundary layer structure occurring at (or very near) the terminus of the liquid film. The basic analysis, however, shows considerable promise. Even the complex case where a compressible gas is strongly accelerated over the gas-vapor cooled region can be reduced to the problem of determining the "effective starting point $(x=0)$ for the growth of the boundary layer over the subject wall region. The task that remains, therefore, is to obtain experimental data on the adiabatic wall temperature for a wide range of flow conditions so that the effective starting point can be correlated in terms of the pertinent variatles. Unfortunately, since such data are not now available, the analysis proposed herein for the gas-vapor cooled region cannot be completely formulated.

\section{A.3 The Case Where the Wall is Cooled Exiernally}

For the subject case, the basic analytical problem is that of relating the local values for the wall temperature and the wall heat flux to the main-stream conditions. It 
can be inferred from several mass transfer cooling (and heating) studies that are reported in the literature (e.g., Refs. 51-55) that heat-transfer data for the gas-vapor cooled region should correlate satisfactorily in the following manner. Thus

$$
s t_{g v}=s t_{0}
$$

where

$$
\begin{aligned}
& S t_{g v}=\frac{q_{W}}{G c_{p g}\left(T_{a w}-T_{w}\right)} \\
& \begin{aligned}
s t_{g v}= & \text { the Stanton number for heat transfer for the } \\
& \text { gas-vapor cooled region }
\end{aligned} \\
& \text { st } t_{0}=\text { the stanton number for heat transfer in the }
\end{aligned}
$$

Equation $(A-3 I)$ reduces the present problem to that of determining the stanton number $s t_{0}$ as a function of the distance measurea iubustream from the end of the liquid film. The theoretical methods that were outlined in subsection 3.1 .2 and in subsection 3.3 .1 can be employed to calculate 
that Stanton number*. In that calculation, however, it is important to note that the following boundary condition applies $(51-55) * *$ :

$$
S t_{0}=\infty \text { at } x=x_{2}
$$

The task remains to obtain experimental data for the rate of heat transfer to an externally cooled surface that is located immediately downstream of the terminus of the liquid film so that the validity of Eq. (A-31) can be determined. Although Emmons (20) and Guinn (45) did obtain some experimental data on that rate of heat transfer, the validity of Eq. (A-31) cannot be investigated with those data because neither Emmons nor Guinn determined the adiabatic wall temperature $T_{\text {aw }}$ that enters into the equation. There is, however, an important observation that can be made with reference to the experimental results due to Emmons. That observation is discussed in the following subsection.

* With $F_{C}=1.0$ and $F_{m "}=1.0$

**Guinn (45) developed a semi-empirical analysis for the gas-vapor cooled region. Guinn, in essence, utilized Eq. $(A-31)$ in that analysis. However, he did not recognize that the above boundary condition applied in the evaluation of the Stanton number $S t_{0}$. As a consequence, his analysis is not valid at the leadina edge of the gas-vapor couled region. 


\section{A.4 An Observation on the Data of Emmons}

Emmons (20) investigated experimentally the rate of heat transfer to an externally cooled test section that was located immediately downstream of the liquid-film cooled test section. Those heat transfer rates, which were determined calormetrically at several locations downstream of the film cooled region, were compared with the heat transfer rates that were realized for the aforementioned 1.48-inch convectively cooled test section that was located immediately upstream of the liquid film*. As a result of that comparison, Emmons drew the following two conclusions ( $p .67$, Ref. (20)):

"A large portion of the film coolant vapor forms a mass of cool gas which remains adjacent to the wall for a considerable distance downstream from the liquid film. As a result the heat flux to the wall protected by the vapor is significantly reduced;" and

"Heat transfer rates measured approximately 9.0 in. downstream from the 1 iquid $\mathrm{f} i \mathrm{~m}$ were reduced by 30 to 70 percent from the corresponding values measured upstream from the liquid film. Ammonia, with the lowest boiling temperature (160F) effected the largest heat transfer reductions for the coolants investigated."

These two conclusions imply that the rate of mixing of

* See Fig. 42. 
the relatively cuol boundary layer gases with the mainstream gas is slow. However, the data of Ref. (19) that were discussed in subsection A.2.5 for the adiabatic wall temperature suggest that the rate of mixing is quite rapid. That is implied by the small value for $x_{2}$ (or $\theta_{2}$ ) that was found to result in a satisfactory correlation of the experimental data. That small value for $x_{2}$ suggests that the rate of entrainment of the hot mainstream gas into the boundary layer (or the rate of growth of the boundary layer) is very rapid for the subject wall region. The data presented in Fig. A.2 show that at a point approximately 10 inches downstream of the liquid film, the cool boundary layer gas and the hot mainstream gas are essentially completely mixed. There appears to exist, therefore, some incorsistency in the aforementioned conclusions that were drawn $b_{j}$ Emmons and the experimental data that were reported in fief. (19). 4.:1aver, an explanation for that inconsistency can be suggested: It was argued in subsection 4.4 .5 that the measured rates of heat transfer to the 1.48-inch convectively cooled tast section were apparently about 40 percent higher than those that would be predicted for fully-developed tube flow at the sa.:e bulk flow conditions. Moreover, it was argued that pert (or perhaps all) if that difference could have been due to a discontinuous thermal boundary condition at the leading edge of the 1.48-inch test section. For the convectively cooled test section immediately downstream of the end of the 
liquid film, however, there would be a continuous transition in the wall temperature from the end of the film cooled test section to the gas-vapor ccnled region. Therefore, because of the fundamental difference between the thermal boundary conditions for those two test sections, it would appear that the aforementiolied conclusions that were drawn from the direct comparison of the heat transfer rates for the two test sections may not have been completely valid. If, in fact, an isothermal-wall boundary condition for the 1.48inch long test section would have resulted in heat transfer rates that were 40 percent lower than those that were actualiy realized, then the comparison of the experimental data for the two convectively cooled test sections would have showed that the heat transfer rates measured approximately 9.0 downstream of the liquid film would have been reduced by 0 to 20 percent from the corresponding values measured up. tream from the 1 iquid film, as compared to the 30 to 70 percent reduction that was quoted above from Emmons (20). Trat particular result would appear to be in agreement with the data that were discussed in Subsection A.2.5. However, it cannot be argued conclusively that the significant conclusions that were drawn by Emmons with respect to the subject wall region are in error. That question cannot be completely answered until considerable more experimental data are obtained under well controlled experimental conditions for the gas-vapor cooled wall region. Perhaps the 
foregoing discussion will serve as an impetus for that research. 


\section{APPENDIX B \\ DESCRIPTION OF APPARATUS \\ B.1 General Description}

The gas source employed in the subject investigation was an air blowdown wind tunnel in which a portion of the air flow was heated by combustion with a liquid fuel. The principal features of the apparatus were that it had a wide range of operating conditions, i.e., flow rate, gas temperature and pressure, reproducibility of operating conditions from run to run, low drift about the operating point over run times up to one hour, and was safe and reliable enough to work with in the test cell during operation of the apparatus.

Figure B.l presents a photograph of the basic configuration of the experimental apparatus for that phase of the investigation where the gas stroam velocity was essentially constant over the length of the wetted test plate. Figure B.2 presents a similar photograph of the basic configuration that was employed in that phase of the iniastigation where the gas stream was accelerated over the wetted test plate. The various components of the apparatus are described as follows. The numbers refer to ejther Fig. B.I 

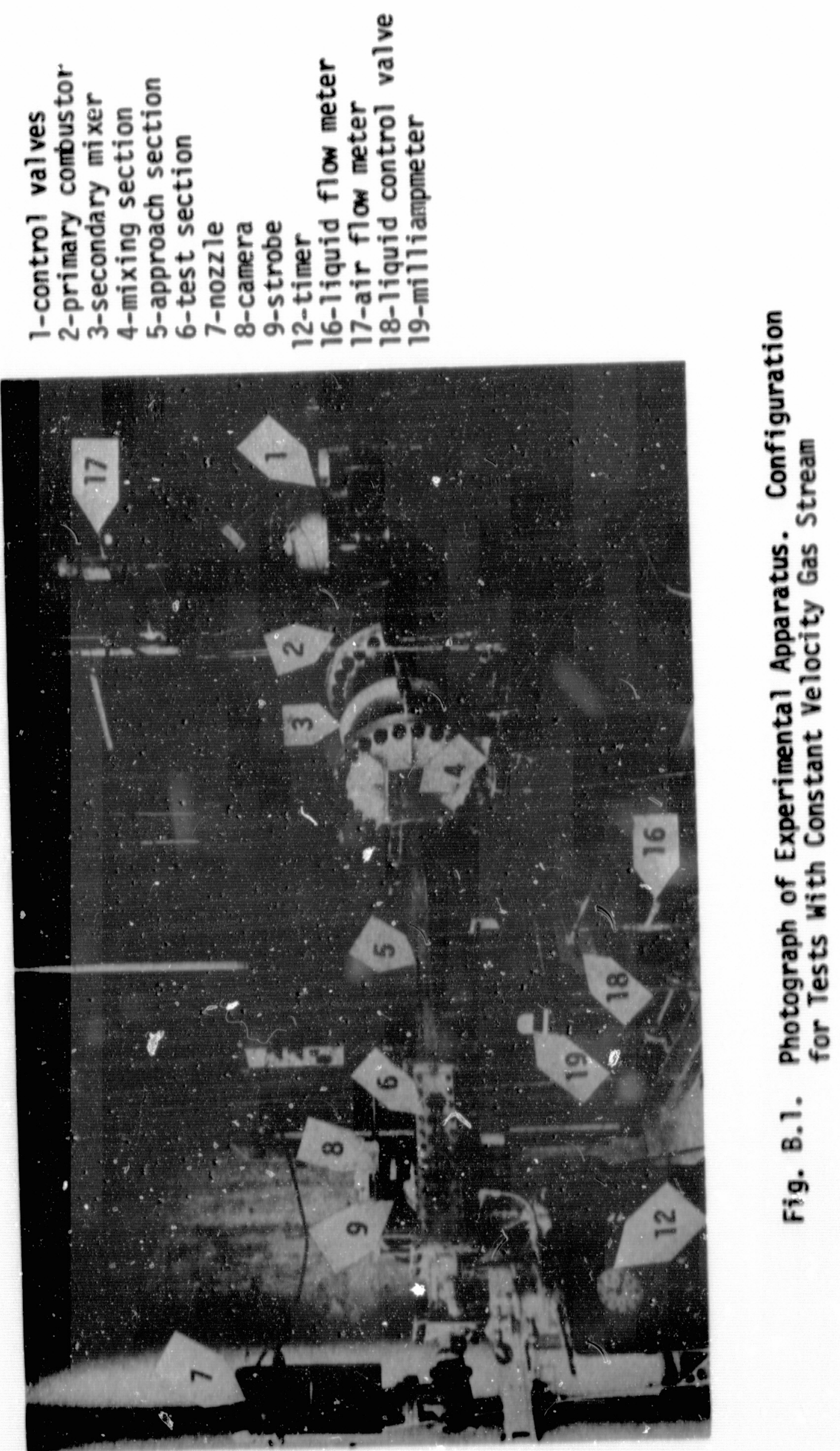

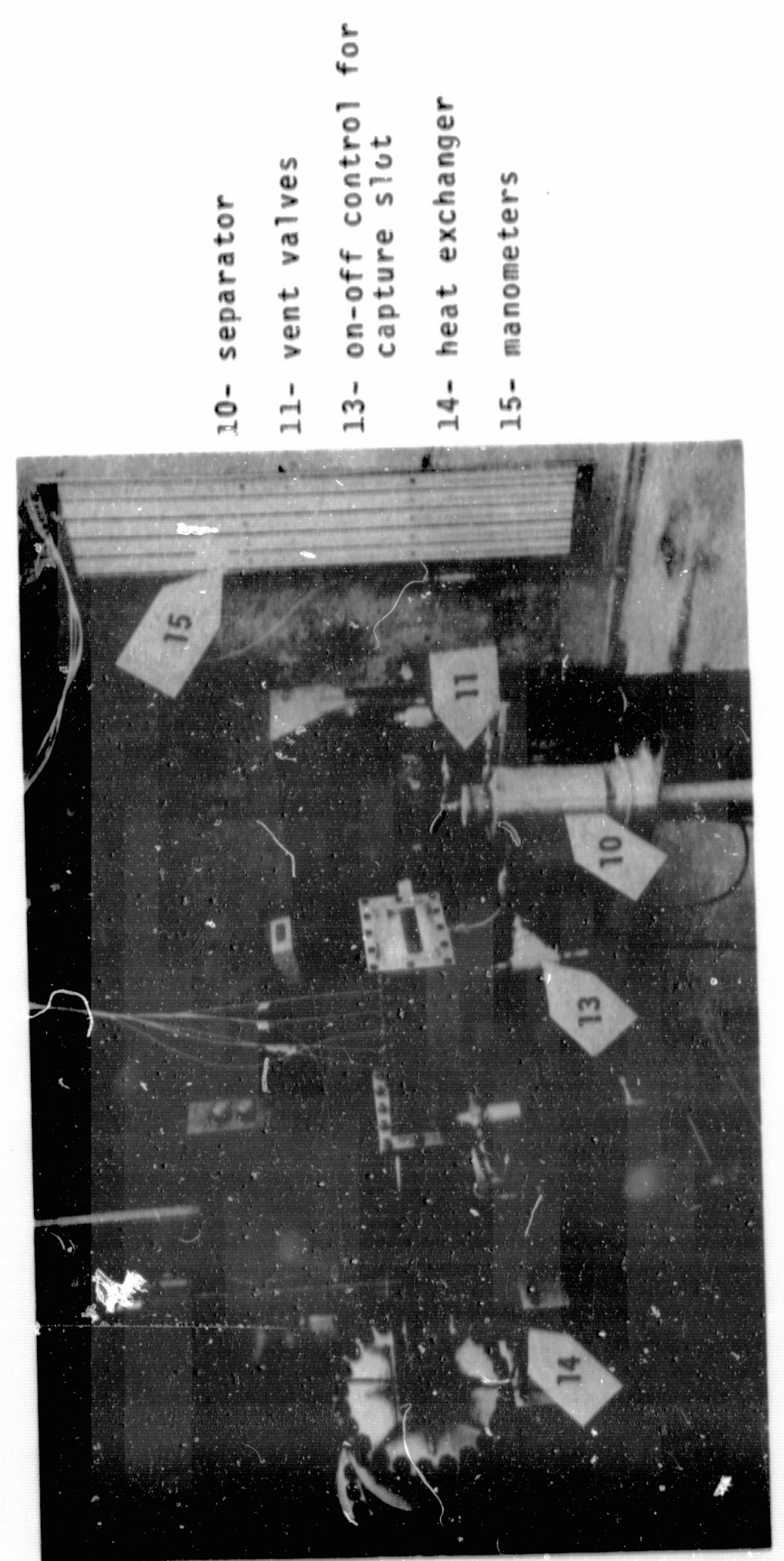

\begin{tabular}{l}
5 \\
0 \\
5 \\
5 \\
5 \\
0 \\
$\frac{5}{6}$ \\
5 \\
\hline
\end{tabular}

究

단

\%

ह。

है

5

ะั

⿶凵

แ

芺管

sn

\%

흔

한

을 응

등운

๓่

,

"ேं 
or Fig. B.2.

(1) The air and fuel flow rate control valves. A separate value was utilized to control (a) the air flow rate to the primary combustor, (b) the air flow rate to the secondary mixer, (c) the fuel flow rate to the primary combustor for ignition, and (d) the fuel flow rate to the primary combustor during full-scale operation.

(2) The primary combustor wherein a high-temperature combustion zone was established.

(3. The secondary mixer wherein diluent air was added to the hot gases from the primary combustor so as to obtain the desired gas stream temperature in the experimental tunnel.

(4) The 10-inch long mixing section which contained one 10-mesh screen and one 30-mesh screen that were spaced 10 inches apart, and which reduced the non-homogeneities of the gas stream flow substantially.

(5) The 36-inch long adiabatic approach section.

(6) The experimental test secticn which contained the film cooled test plate.

(7) The variable area nozzle which controlled the gas stream pressure for a given gas stream Reynolds number.

(8) The $35 \mathrm{~mm}$ Nikkorex camera that was employed to photograph the surface characteristics of the liquid film. The camera lens was a Micro-Nikkar f55mm lens with a close-copy adapter. 
(9) The stroboscopic light source that was employed in conjunction with the camera (8). The strobe had a flash duration of 3 microseconds.

(10) The cyclone separator that was empioyed to separate the liquid and the gas phases that were withdrawn at the capture slot. The details of the separator design is given in Appendix B. 4.

(11) The vent values that controlled the rate at which the gas phase was vented to the surroundings (and, therefore, the rate at which the gas phase was withdrawn at the capture slot).

(12) The electronic timer that was employed to measure the sample time for the mass transfer measurements.

(13) The 500 psia air solenoid valve that served as the on-off control for the capture slot. The activation of the electric solenoid valve resulted in the flow of a high pressure jet of air in the withdrawal passage in the reverse direction through the capture slot into the test section, thereby stopping the capture of the liquid film on the test plate. This solenoid valve and the electric timer (12) were controlled simultaneously so that the length of time that the gas-liquid mixture was withdrawn from the test section through the capture slot was accurately controlled. (14) The heat exchanger that was employed to preheat the liquid phase before it was introduced onto the test plate. The heat exchanger consisted of two 3/4-in. 0.D. stainless 
steel tubes of length 36 inches with a calrod heater located along the centerline of each. Each calrod had a maximum capacity of 1000 watts, and the electrical energy that was supplied to each was controlled by a variable reostat.

(15) The mercury manometers that were employed to measure the streamise static pressure profile for those experimental tests in which the gas flow was accelerated over the wetted test plate.

(16) The film coolant flowmeter $r$ was employed to measure the rate at which the coolant was introduced onto the test plate. The flowmeter was a $1 / 8$-inch Potermeter. The frequency output of the flowmeter was displayed to the operator by the milliampmeter (19) and recorded on a stripchart recorder.

(17) The air flow meter. The total rate of air flow through the experimental apparatus was measured with a Brooks turbine type flow meter (model HP-321-6). The frequency output of the flow meter was displayed on a milliampmeter in the control room and was simultaneousiy recorded on a stripchart recorder.

(18) The precision needle valve that was employed to control the rate of film coolant flow through the heat exchanger and then onto the test plata.

(19) Milliampmeter utilized to monicor the coolant flow rate. 
Figure B. 3 shows the control room. The primary components of the control room are as follows. The numbers refer to Fig. B.3.

(1) The automatic sequencer that was utilized to start the hot gas generator. The operation of the sequencer is discussed in greater detail in Appendix B.2.

(2) The 3 small volume Nullmatic pressure regulators that were employed to control the flow rates through the three Annin Domotor control valves: (a) the primary air control valve, (b) the secondary diluent air control valve, and (c) the primary fuel control valve for full-scale operation. (3) The stripchart recorder that was employed to continuously monitor the gas stream temperature.

(4) The Bourdon tube pressure gage that was employed to continuously monitor the gas stream pressure.

(5) The variable reostat that was utilized to control the temperature for the liquid coolant at the point of injection.

(6) The militiampmeters that continuously minitored the peritinent system flow rates and system pressures. These various parameters were also recorded on stripchart recorders located in an adjoining room.

\section{B.2 The Gas Generatur}

Figure B.4 nrosonts a simplified sectinn=l drawing of the gas generator portion of the experimen:al apparatus. 

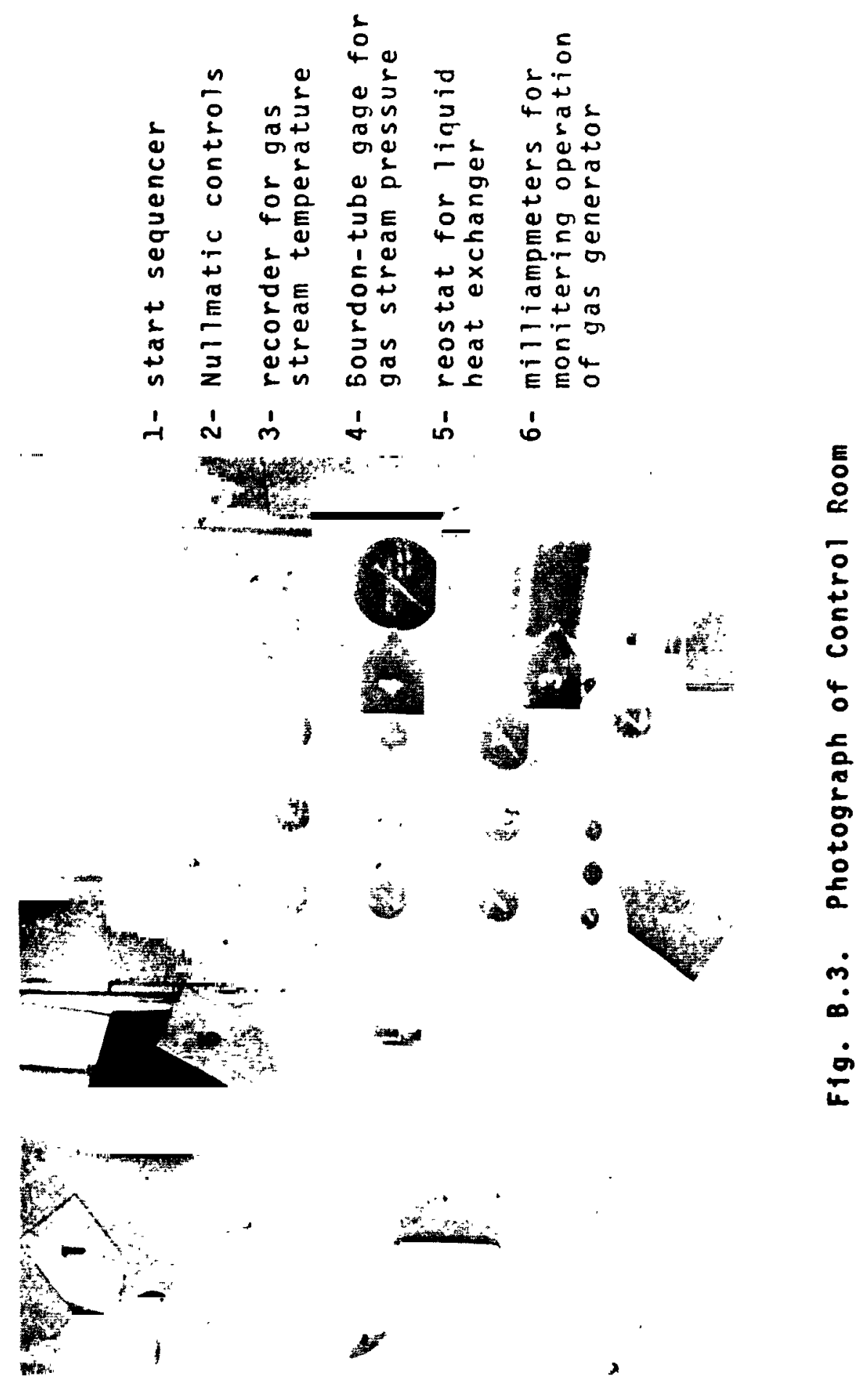

통

1

1

1

I

we.

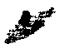




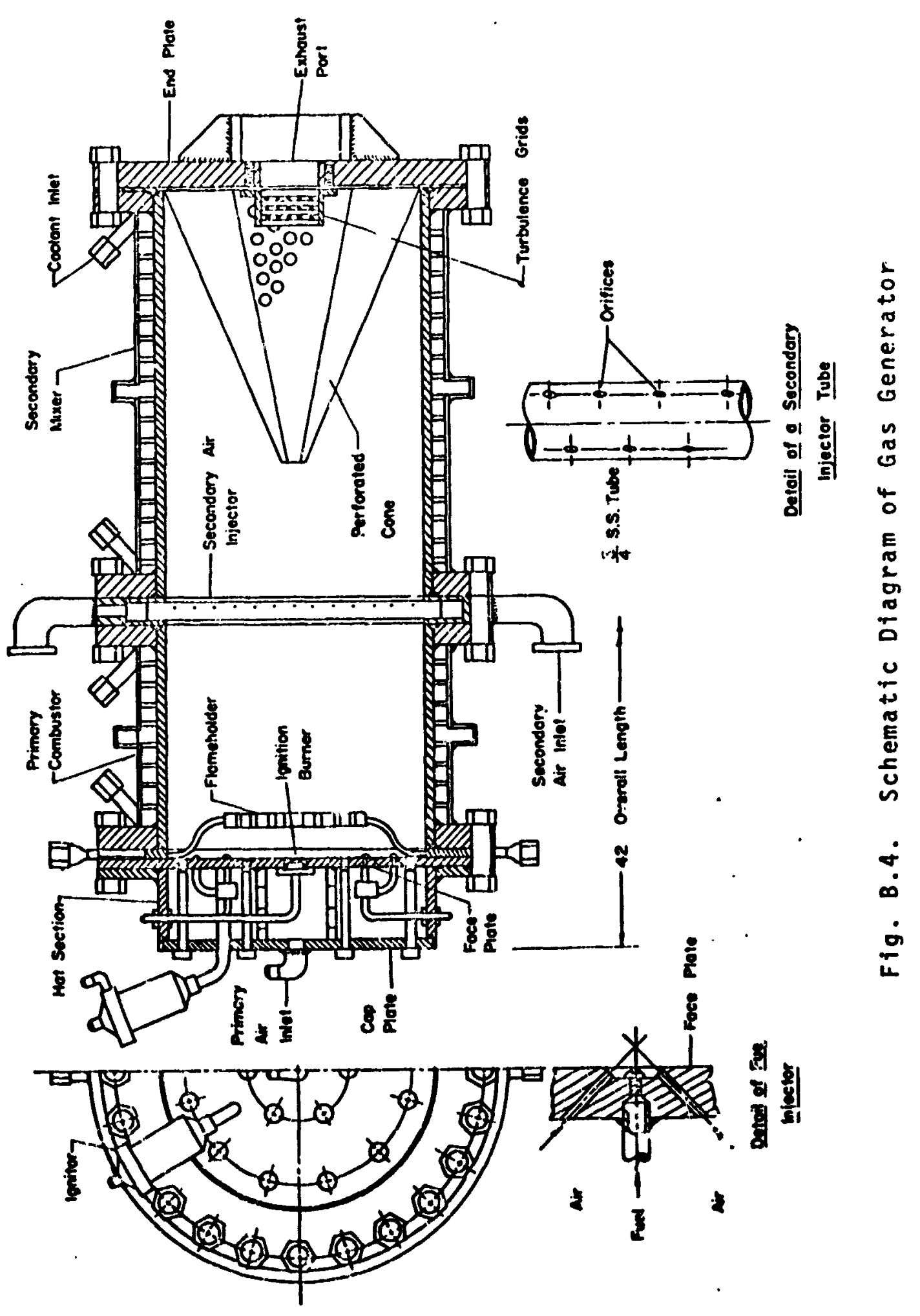


Table B.l iists the components of the gai generator and describes the function of each.

Tabic B.1

Components of the Gas Generator

\section{Component}

Primary Injector

Flameholder

Primary Combustor

Secondary Injector

Perforated cone and turbulance grids

\section{Function}

ic inject the fue: and air to be burned into the irimary combustor.

T: $m_{i} i x$ the fuel aird air after injection and provide turbulence to hold the flame.

To provide a contained volume for combustion.

To inject diluent. air to reduce temperature of worting fluid to the desired value.

To thoroughly mix th diluent air with the combistion products from the primary combustor in order to establish a uniform temperature throughout the gas flow. 
Table 9.2 presents the output characteristics of the gas generator for the overall allowable range of operation.

$$
\text { Table B.2 }
$$

Output Characteristics of the Gas Generator

Parameter

Overall Range

Gas Stream Pressure, psia

Cold flow

Hot flow

Gas Stream Temperature, $R$

Cold flow.

Hot flow

Gas Flow Rate, $1 \mathrm{~b} / \mathrm{sec}$

Cold flow

Hot flow

Fuel Flow Rate, Ib/sec up to 400

$50-350$

500

$750-1100$

up to 15

$0.75-10.0$

$0.02-0.25$ 
The air flow entering the gas generator was divided such that approximately 30 percent of the total flow entered the primary combustor to be burned with the fuel while the remaining 70 percent of the air flow was injected into the secondary mixing chamber. Approximately five percent of the fuel was injected from the ignition fuel injector located in the center of the primary injector while the reinaining fuel was injected fro: the main portion of the primary injector. The fuel and the primary air (representing approximately 200 percent excess air) were burned in the primary combustor at a temperature of $2500 \mathrm{~F}$ with a thermal efficiency of approximately 50 percent. Smooth stable combustion rather than high thermal efficiency was desired. The low thermal efficency was the result of insufficient volume in the primary combustor to permit complete combustion of the fuel.

The primary injector comprised three principal parts, as shown in Fig. B.4, namely, the face plate, the hat section air manifold and the cap plate. Ninety-five percent of the methyl alcohol fuel was injected through two concentric rings having a total of 30 separate fuel injector buds located on the face plate (see detail in Fig. B.2). The face plate was fabricated from a circular piece of stainless steel plate $\frac{1}{2}$ in. thirk on each side of the fuel injectors were two 0.100-in. diameter injector orifices for air directed at $45^{\circ}$ to the direction of the fuel flow, the combination forming a triplet. The primary air was introduced 
into the injector manifold by a singie 1.0-in. tube fitting. The fuel was manifolded by $\frac{1}{4}-i n$. O.D. stainless steel tube to six clusters of heavy wall tubing, each with tubes welded into the back of the face plate; these clusters fed fuel to the 30 fuel injectors. The ignition fuel which was required as part of the start sequence was manifolded directly to the center of the face plate and was injected through four small. orifices against a copper splash ring. Air for the combustion of the ignition fuel was supplied through a ring of small orifices concentric to the ignition fuel injector orifices.

The flameholder consisted of a central perforated disc of stainless steel supported by eighteen $\frac{1}{4}-i n$. tubes of the same material which ran radially to an outside ring. Cooling water was introduced through a 1.0-in. tube fitting located $180^{\circ}$ from the output fitting. The flameholder was of all welded construction. In the assembled gas generator the flameholder was sandwiched between the primary injector and the primary combustor.

The primary combustor and secondary mixer chambers were of similar design and construction, differing only in length. The former was $10 \mathrm{in.}$ long and the latter was $20 \mathrm{in.}$ long. The inner wall was a section of 13-in. I.D. mild steel pipe having a wall thickness of $\frac{1}{2}$ in. Welded at each end of the pipe section were one-inch thick mild steel flanges with twenty-four l-in. nominal bolt holes equispaced on a bolt 
circle of $20 \mathrm{in}$. The coolant passages were formed from extruded lead stripping with a $\frac{1}{4}-i n$. by $3 / 4-i n$. cross section which was wrapped in a spiral around and soldered to the pipe. The outer jacket of the coolant passages was formed from half-sections of 1/8-in. wall, 16-in. I.D. stainless steel tube with an expansion joint located in the middle. The jacket was sealed all around by weldments. Inlet and outlet fittings for the coolant, which was water, were welded over holes in the outer jacket and located at either end of the lead spiral. All cooled parts were incorporated in a single pass coolant path.

The secondary air injector was fabricated from seven parallel 3/4-in. 0.D. stainless steel tubes set in a 13-in. I.D. outer ring which served as a distribution manifold. Each tube was drilled with a $0.125-i n$. 0.D. hole every $3 / 8$ in. along its length in such a way that two rows of orifices were formed directed downstream $45^{\circ}$ off from the direction of gas flow from the primary combustor (see detail in Figlire B.2). This arrangement enhanced turbulent mixing immediately downstream from the injector tubes. The injector tubes were fed from the outer ring which in turn was fed secondary air through a $2-i n$. pipe fitting located on the outermost part of the ring.

Downstream of the secondary air injector was located a stainless steel cone perforated with $k_{2}-i n$. diameter holes. The cone pointed upstream and was clamped between the end 
plate and the aft flange of the secondary mixer chamber. Turbulence grids were mounted just ahead of the $2-i n . \times 4-i n$. exit port located in the center of the end plate. The perforated cone and turbulence grids induced mixing of the secondary and primary gases. The end plate was fabricated from l-in. thick mild steel plate reinforced by gussets welded on the outside.

The flanged sections of the gas generator were bolted together with twenty-four 1-8 studbolts. The gaskets between the separate sub-assemblies were either $1 / 32-i n$. thick aluminum or $1 / 16-i n$. thick composition asbestos. Concentric serrations to aid sealing at the gaskets were machined on the injector face plate, the end plate and the flanges; no serrations were present on the flameholder or secondary air injector.

Operation. Figure B.5 presents a simplified schematic diagram of the flow system for the experimental apparatus. The air used in the experiments was drawn from the high pressure supply tanks which comprise the blowdown air supply for the Jet Propulsion Center. The tanks were charged with a maximum pressure of 2400 psig. The compressed air was delivered to an isolating valve located on the roof of the test cell by a pipeline from the supply tanks. The isolating valve on the roof was a 2-in. 1500 ASA Annin pneumatically operated open/closed valve. It was present as a safety feature to isolate the high pressure air from the 


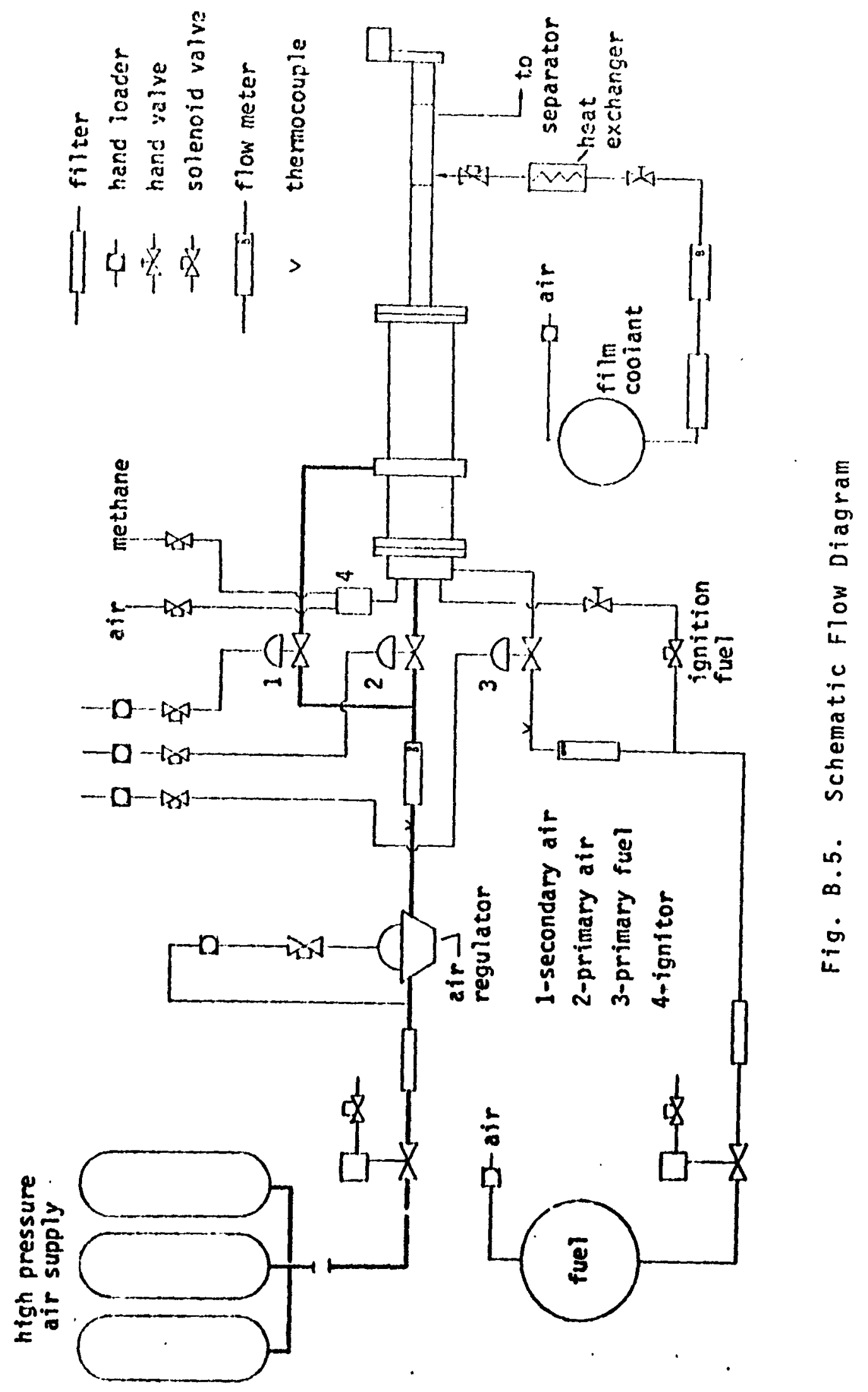


test cell housing the apparatus in the event of a severe mechanical malfunction of the gas generator.

The air supply was passed through a Fluid Dynamics high pressure air filter. The filter element removed particles from the gas stream that were larger than 10 microns. The air was then reduced from the supply line pressure by a 2 -in. Grove Powereactor dome reguilator located immediately downstream from the air filter. The Grove regulator was loaded by means of a small volume Grove pressure regulator. The volume flow rate of air was measured with a 2-inch Brook's turbine flowmeter located after a straight section of pipe dounstream of the regulator. The weight flow rate of air was determined from the frequency output of the flowmeter and the static pressure and temperature of the air measured immediately ahead of the flowmeter. The static pressure was measured with a Wiancko static pressure transducer and the temperature was measured with an iron-constantan thermocouple. The three aforementioned parameters were recorded during an experiment on Brown self-balaricing stripchart recorders.

The air flow to the gas generator was controlled by two Annin Domotor valves, a 2-in. valve for the secondary air and a 3/4-in. valve for the primary air. Control air for these pneumatic valves as well as for other pneumatic functions in the system was supplied at pressures of 400 , 100, 45, and $30 \mathrm{psig}$ from efther Grove Powereactor 
regulators or Nullmatic small volume regulators.

The methyl alcohol fuel was stored in 55-gallon shipping drums and transferred to two $6-\mathrm{cu}$. ft. run tanks located outside the building housing the test cell. The fuel was pressurized with air by means of a small volume Grove pressure regulator. The fuel tanks were isolated from the building by a pneumatically operated Annin valve located beneath the fuel tanks. The flow rate of fuel was measured with a 1/8-in. Potter turbine flowmeter, the frequency output of which was recorded on a Brown recurder. The fuel flow rate was controlled by means of a modified $3 / 4-i n$. Annin Domotor valve which was rebuilt to control with precision the relatively small flow rate of fuel.

Before the gas generator was started in hot flow, the air pressure and fuel tank pressure were set to the desired value. Coolant water was supplied to the gas generator by a Horthington turbine pump driven by an electric notor. The three Annin Domotor valves were pre-set using Nullmatic control regulators (see Fig. B.3) to give the proper start flow rates of primary air, secondary air and fuel.

The ignition source for the gas generator was a small combustor (see Fig. B.4) which burned gaseous methane and air ignited by d:-? ej:rk pluss.

The ignition sequence was controlled by an automatic sequencer (see Fig. B.3). The ignition sequence was as follows: 
1. The methane-air ignitor was started.

2. When the ignitor reached the desirad temperature, the automatic sequence. was initiated.

3. The ignition fuel and primary air valves opened simultaneously and a pilot flame was established in the center of the primary injector off of the ignition fuel injector.

4. Two seconds later, the primary fuel and secondary air valves opened simultaneously.

5. The ignitor combustor was shut down and a second or two later the mainstage ignition was arcomplished by the pilot ignition flame.

The whole automatic start sequence required approximately six or seven seconds. Once mainstage ignition was accomplished (usually at reduced flow rates), the operator brought the gas generator up to the desired run point using the controls for the Annin Domotor valves.

During operation of the gas generator, monitoring of the various pressures associated with the system was accomplished either by Bourdon tube pressure gages or Wiancko pressure transducers. The outputs of the latter were displayed to the operator by milliampmeters* and were also recorded on Brown stripchart recorders. Flow rates of fuel and air were also displayed to the operator by milliampmeters* and recorded on stripchart recorders. The temperature of the gas leaving the gas generator was measured by two chromel-alumel thermocouples located immedfately downstream of the end plate at the entrance to the approach section. The output of one of the thermocouples was

*Shown in Fig. B.3. 
displayed to the oparator on a direct reading milliampmeter while the other output was recorded on a siripchart recorder. The pressure of the gas in the lead section was the value employed to set the pressure of the run point. The gas stream pressure was measured, displayed and recorded in the manner as previously described.

After the desired operating point had been achieved, approximately ten minutes were required for the test section to achieve thermal equilibrium. Only then were the experiments begun. Luring run point operation the gas generator exhibited a random drift about the run point of $\pm 20 \mathrm{~F}$ of gas stream temperature and \pm 2 psi of gas stream pressure.

\section{B.3 The Test Section and Test Plate}

Figures $B .6$ and 8.7 present sectional drawings of the experimental test section. The basic internal dimensions of the test section were 2 in. by 5.5 . in. cross section by $18 \mathrm{in}$. long. The test section consisted of 4 side wal's and the wetted test plate. The side walls were made from 9/16in. thick stainless steel plate and the upper and lower walls were made from 3/4-in. thick stainless steel plate. The test plate was $z-1 n$. thick stainless steel piate. All of the internal wall surfaces were surface ground. A single Pyrex window of dimensions $11 / 16 \times 1-5 / 16 \times 9-7 / 8 \mathrm{in}$. long was mounted in the top wall of the test section, and 6 circular 1-in. O.D. Pyrex windows 9/16-in. thick were equispacejat 2 inches in each side wall. All of the Pyrex 


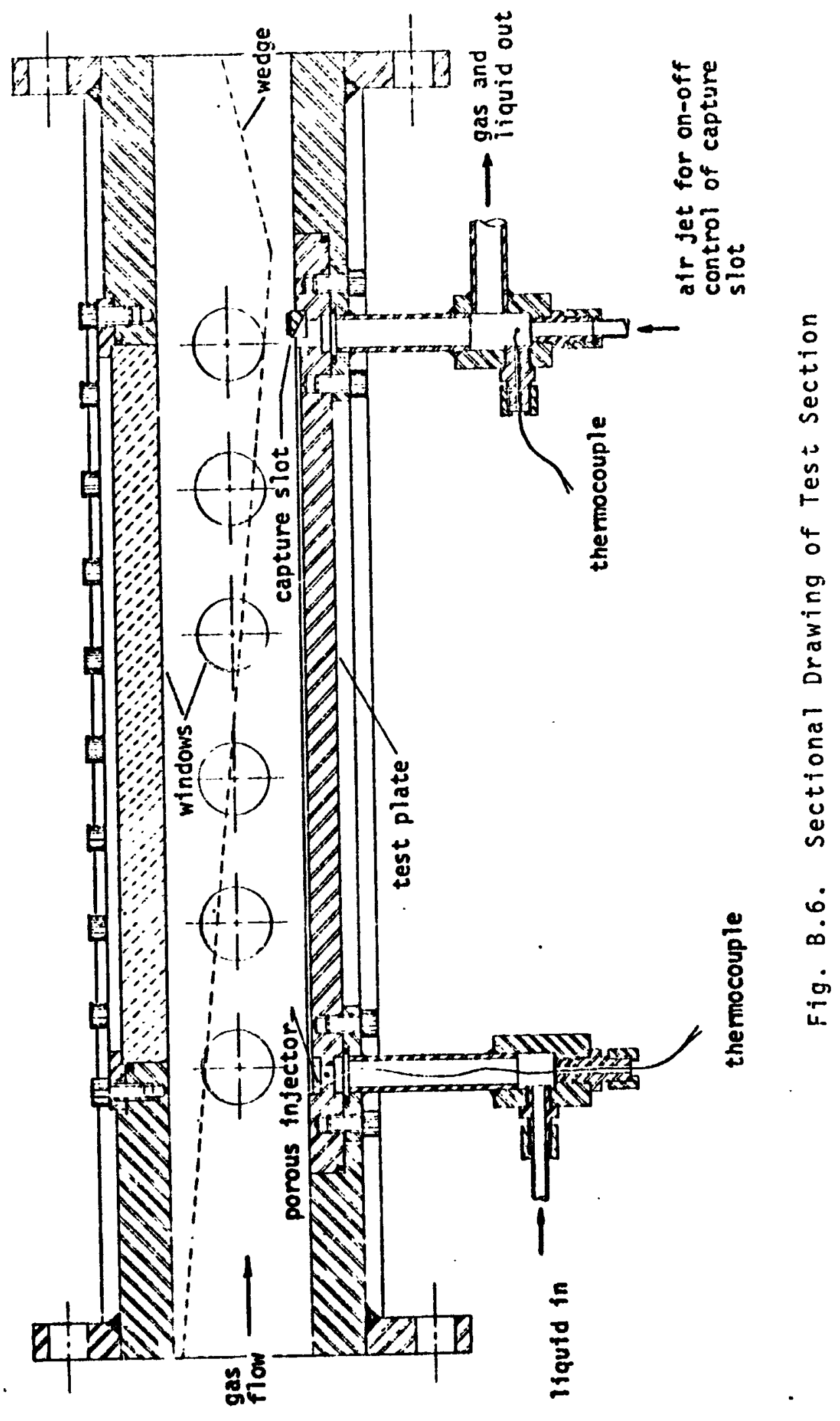




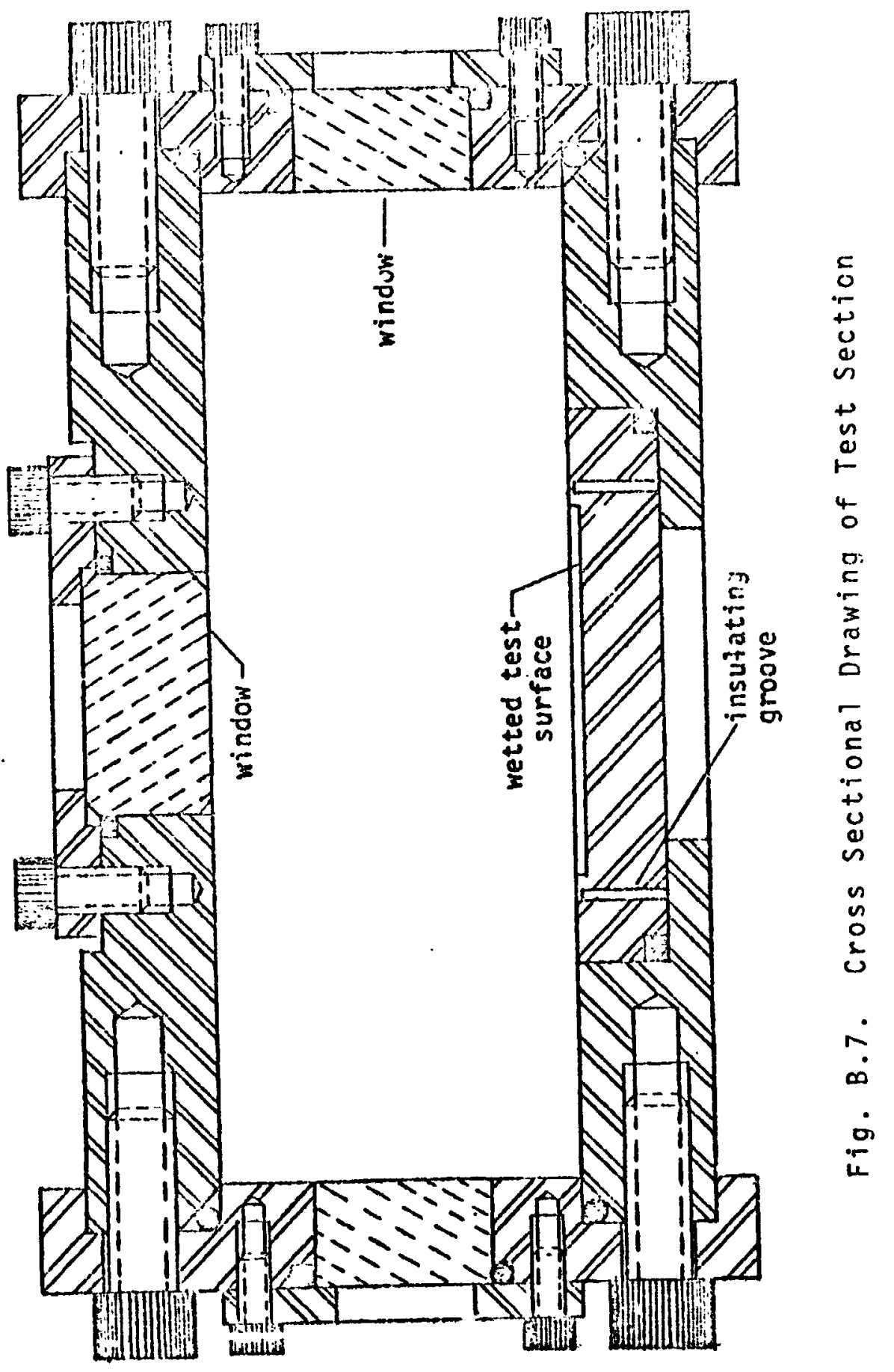

I
1
]
]

]

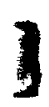

1

]

I

) 
windows were sealed by Viton-B o-rings, as indicated in the drawings.

The dashed line shown in Fig. B.6 represents the profile for the aluminum wedge for that phase of the investigation where the gas stream was accelerated over the wetted test plate.

Figures B. 6 and B.7 show that the test plate to which the liquid film was confined dropped into a recess that was machined into the bottom wall of the test section and "floated" on an o-ring seal. The liquid injection and liquid withdrawal manifolds are shown bolted directly to the test plate in Fig. B.6. The passage for the high-pressure air jet that was employed for on-off control of the knifeedge capture slot is shown in the withdrawal manifold. The locations of the two chromel-alumel thermocouples that were employed to measure the inlet and outlet liquid temperatures are indicated in the figure. Figure B.6 shows the porous stainless steel injector element that was constructed from a 1/8-in. thick by 2-in. long piece of "regimesh". The cross-sectional view of the knife-edge capture slot is also shown in Fig. B.6. Figures B.6 and B.7 show the 2-in. wide by $0.060-i n$. deep trough that was machined into the test plate to which the liquid film was confinar The 2-in. by 10-in. wetted test surface was hand polished. Figure B.7 also shows the two $0.060-i n$. wide insulating grooves that were cut into the underside of the wetted test plate so as 
to substantially reduce the lateral conduction of heat through the test plate to the liquid film.

The four side walls of the test section were bolted together with 3/8-24 stainless steel bolts. Viton-B cord was employed to seal the test section along each of the four edges. Two flange pieces were welded to each of the four test section walls so that a continuous flange surface existed at each end of the test section when the test section was assembled. The upstream approach section and the transition section between the test section and the exhaust nozzle were bolted to those flanged ends of the test section with $\frac{1}{2}-20$ bolts. Extreme care was taken to align the internal flow surfaces for the test section with those for the approach section.

Figure B. 8 presents two photographs of the assembled test section. Figure $B .8(a)$ shows the configuration for that part of the investigation where the gas stream velocity was constant. The figure shows the location of the $35 \mathrm{~mm}$ camera that was employed to photograph the surface of the liquid film. Figure B.8(b) shows the test section configuration for the studies with an accelerated gas stream. For those experimental tests, the Pyrex window in the upper wall of the test section $i:=: \because 40$ oved so is to have access to the manometer leads employed to measure the static pressure along the aluminum wedge. The tubing for one of the 5 static pressure taps is shown in the figure. 


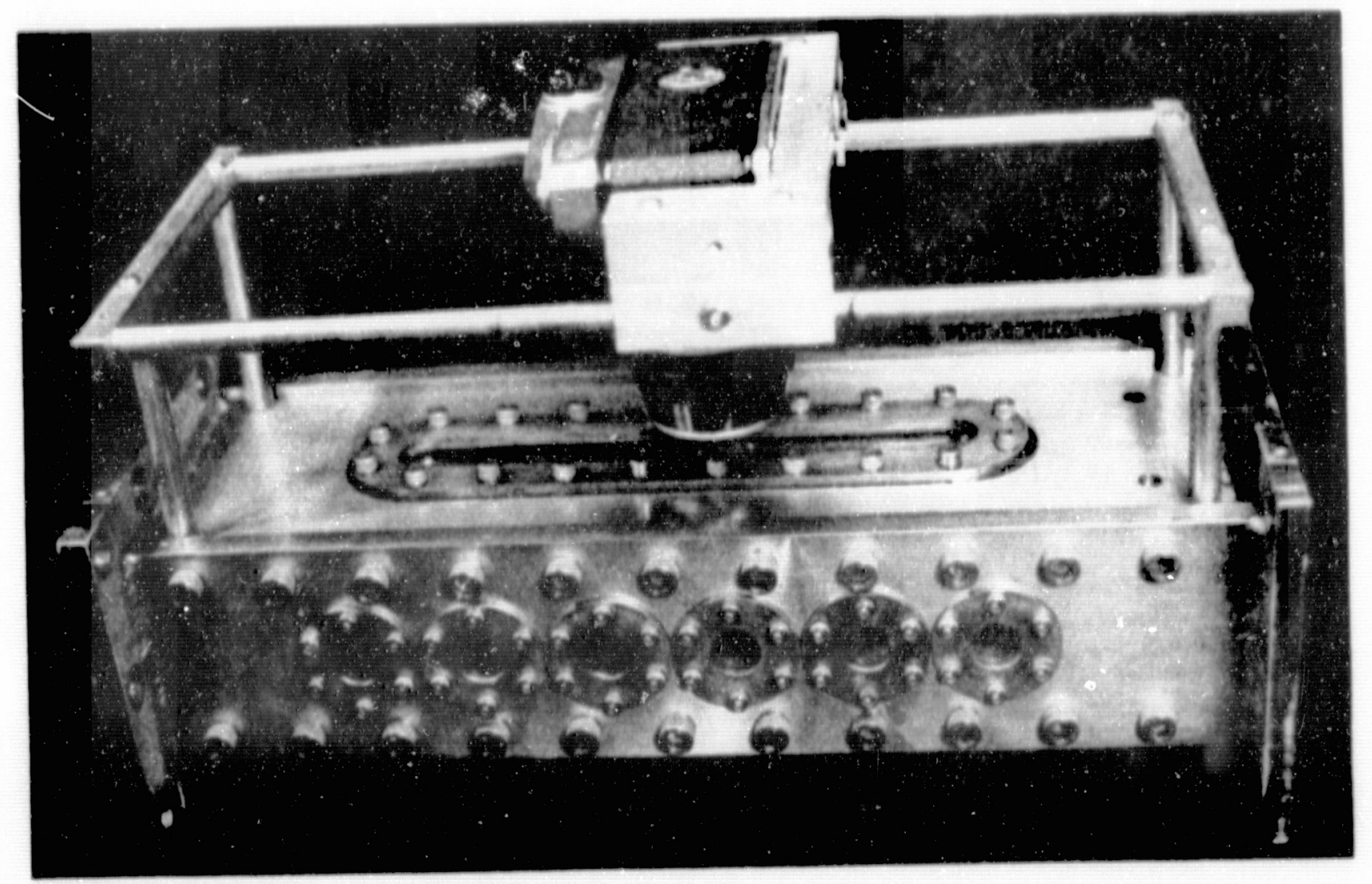

(a) Constant-Velocity Gas Flow

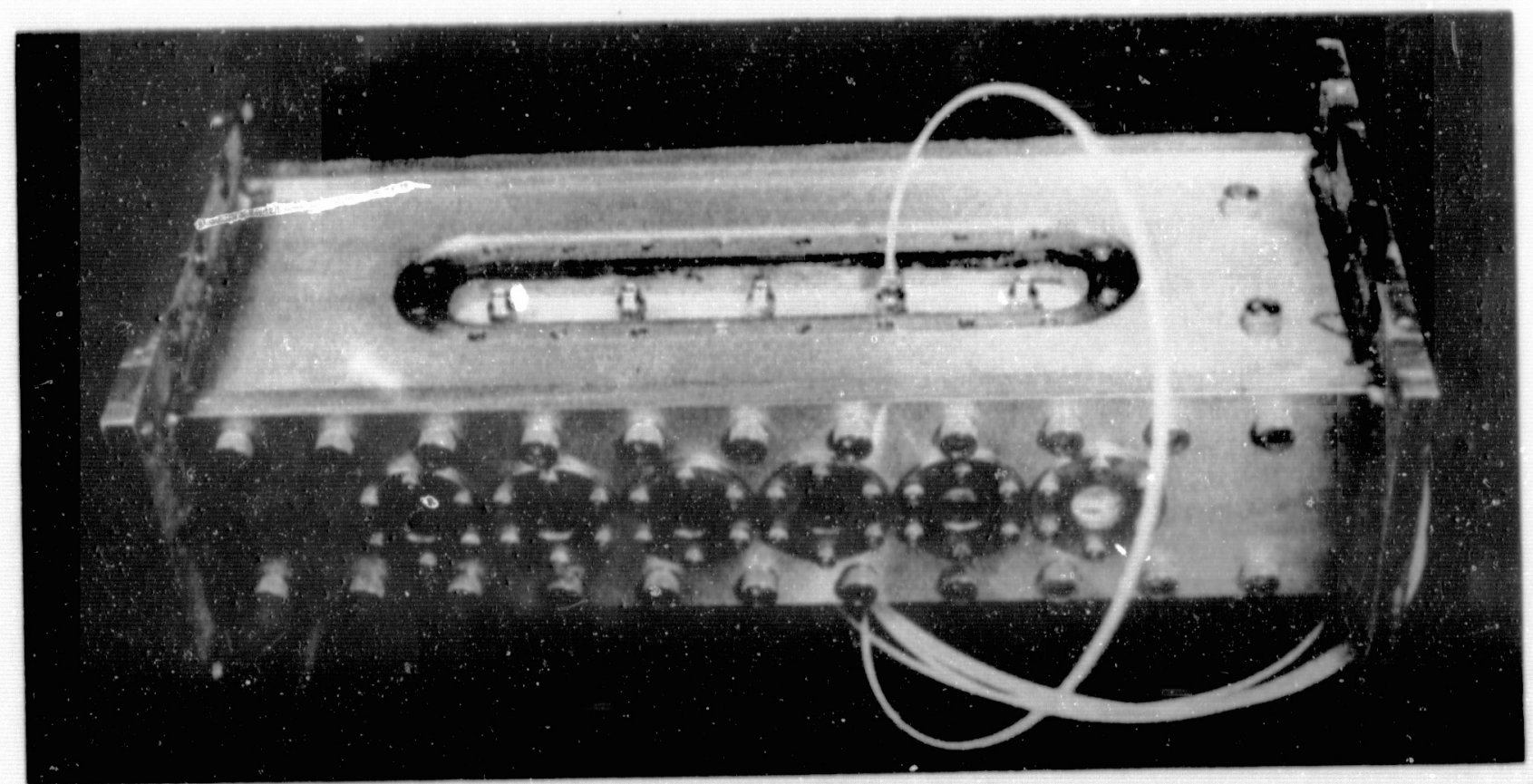

(b) Accelerated Gas Flow

Fig. B.8. Photographs of Test Section 


\section{B. 4 The Separator}

Figure B.9 shows a sectional drawing of the cyclone separator that was employed to separate the liquid and gas phases. The gas and liquid mixture that was withdrawn from the test section through the capture slot entered the separator at top of the main chamber in a tangential direction through a $\frac{1}{2}-i n$. O.D. stainless steel tube. The centrifugal forces on the liquid drops caused them to form a film on the outer wall of the separator and to eventually drain to the lower chamber. The gas exhausted to the surroundings through the center chamber. The rate at which gas was vented to the surroundings (and, therefore, the rate at which it was withdrawn at the capture slot) was controlled by two precision Hoke needle valves that were mounted on the center chamber.

Figure B.10 shows a photograph of the separator and the support stand. Also shown in the figure is the graduated beaker employed to collect the liquid sample from the separator and the electric timer that measured the sampie time. The on-off control for the capture slot (an electric solenoid with a 500 psia air supply) is shown located under the test section. The aforementioned timer and the on-off control were simultannausij regulated by one of the switches that is shown on the front panel of the support stand for the separator. The procedure for operating the cyclone separator was as follows. First the high-pressure air solenoid 


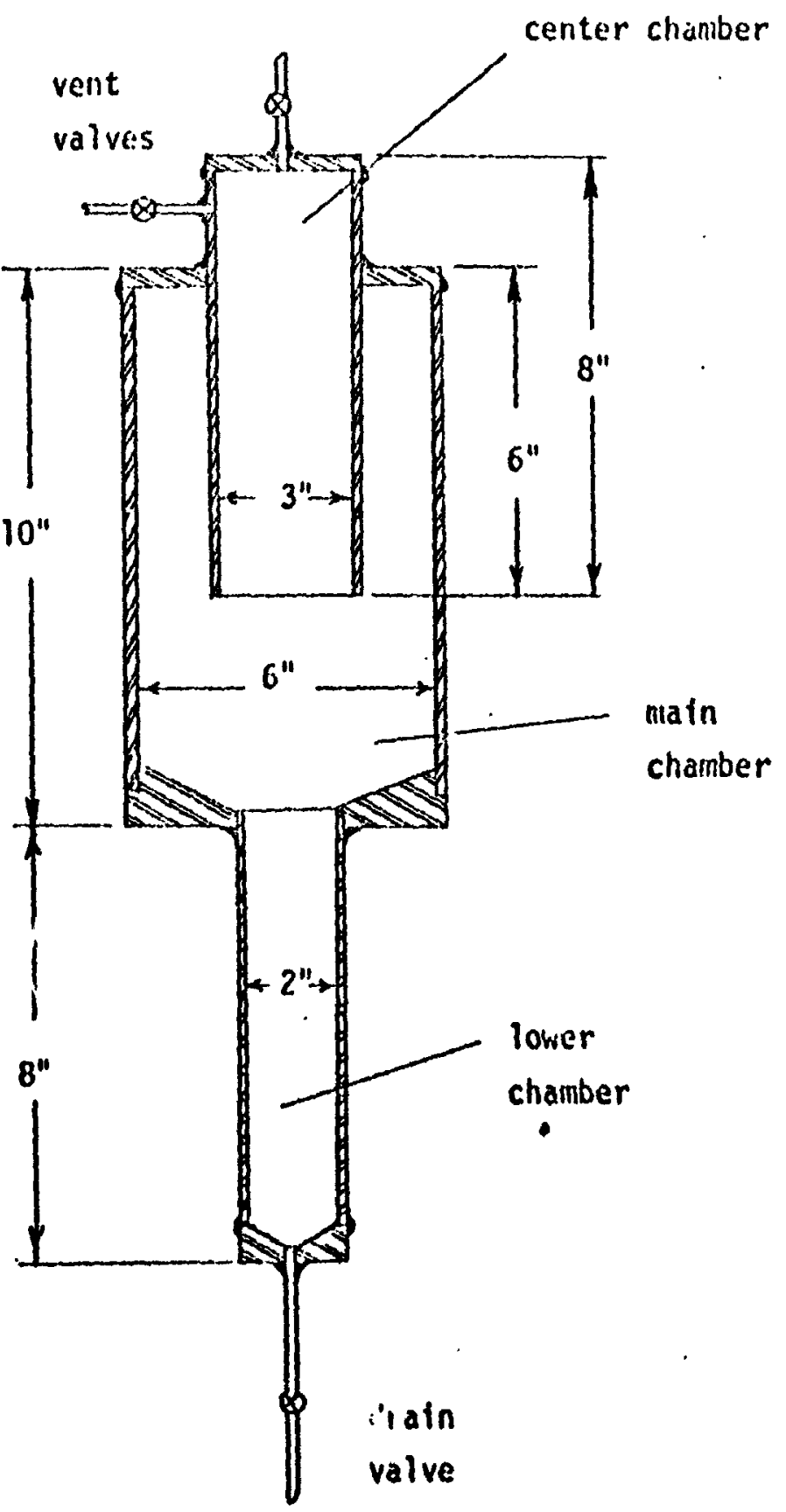

Fig. B.9. Sectional Drawing of Separator 
1

1

1

]

1

]

f

1

f

1

1

1

1

1

Fig. B.10. Photograph of Separator

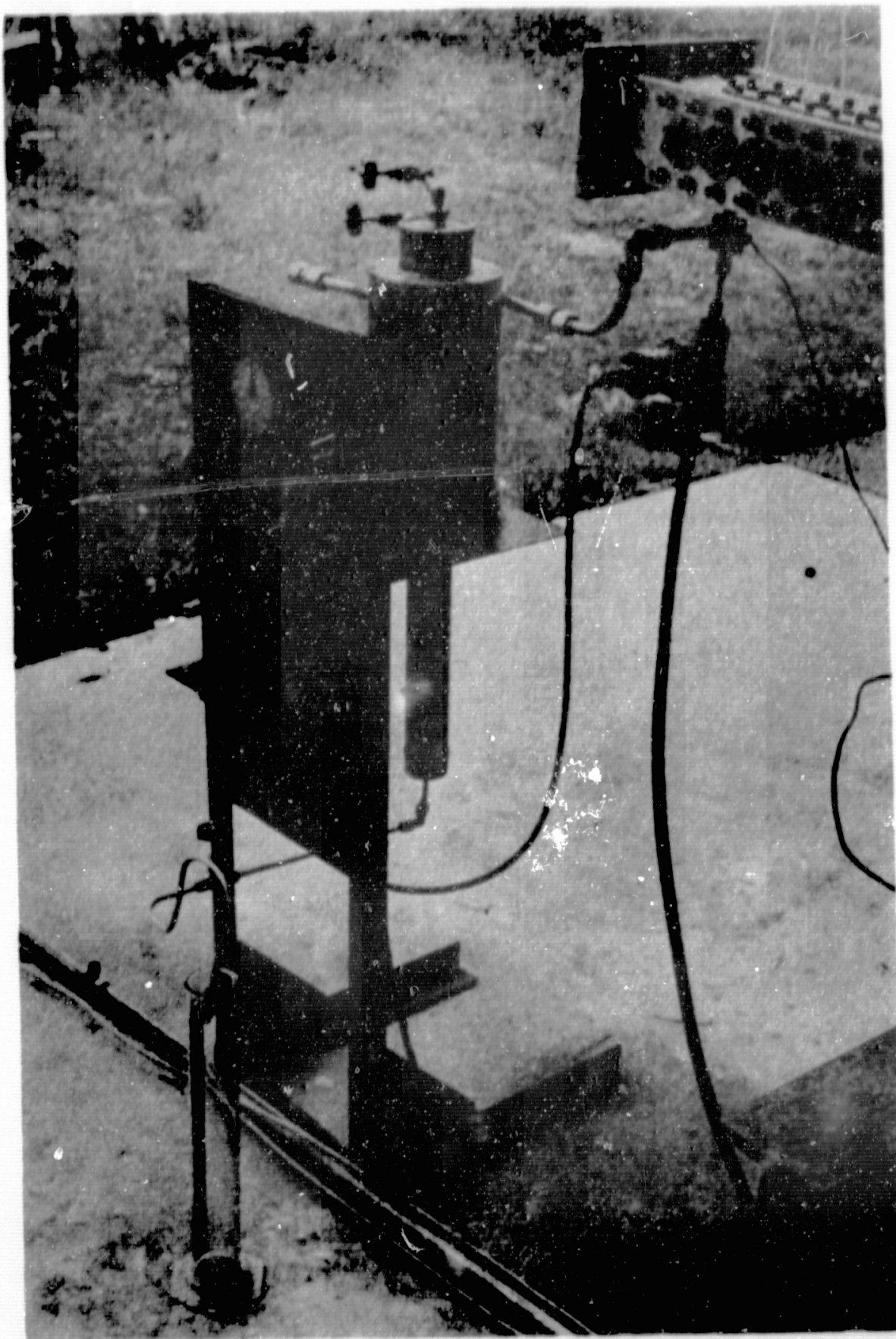

I 
was activated so that no gas or liquid entered the capture slot. Then the bottom drain valve on the separator was opened so that any liquid that remained in the separator was removed. The drain valve was closed. Then the timer was started and the high-pressure air jet was shut off simultaneously, thereby beginning a mass transfer measurement. After a certain length of time, normally one minute, the switch was thrown to both stop the electric timer and to reactivate the electric solenoid for the high-pressure air jet. The drain valve on the bottom of the separator was then opened and the liquid in the lower chamber of the separator was collected in the graduated beaker. The size of the liquid sample that was collected ranged from approximately 100 to $800 \mathrm{ml} / \mathrm{min}$, depending on the rate at which the liquid was injected onto the test plate. The temperature of the liquid in the beaker was frequently measured with a mercury thermometer so as to determine the specific gravity of the liquid at the time of collection. Knowing the specific gravity for the liquid and the volume of the liquid that was collected, the average rate of liquid flow out of the test section during the sample time could be determined. 


\section{EXPERIMENTAL PROCEDURE}

\section{C.1 Pre-Run Preparation}

The pre-run preparation of the experimental apparatus encompassed three major items, namely, (1.) the preparation of the gas generator and instrumentation systems, (2) the calibration of the instruments, and (3) the establishment of the start-up configuration for the entire apparatus prior to the start of an experimental run.

The air storage tanks having a total volume of $2500 \mathrm{cu}$. $f t$. were charged with air to a pressure between 1500 and 2400 psi which was sufficient for operation of the gas generator for a period of from 2000 to 4000 seconds. The fuel tanks were filled with methanol. Also the film coolant tank was filled. Several hours prior to an experiment the instrumentation was turned on to allow for warm-up and stabilization. The camera, if employed, was loaded with film and mounted on the camera support. The strobe unit was checked to ensure proper functioning and was patched into the synchronization system of the camera. The combined system was then checked again to ensure correct functioning. 
The pressure transducers monitoring the operation of the gas generator were calibrated in 5 psi increments around their normally recorded values employing a precision Bolirden tube pressure gage as a standard. The precision gage was feriodically calibrated employing an Ashcroft dead weight tester. The calibration of the turbine flowmeters monitoring the air, fuel, and liquid flow rates was accomplished by means of a 100 cps reference signal available at the Potter flowmeter electronic tachometers. The tachometers supplied a d.c. analog output proportional to the rotative speed of the flowmeter elements. All of the aforementioned pre-run calibrations were recorded on the same stripchart recorders which recordec the parameters during an experiment. The Pottermeter that measured the rate of liquid flow onto the test plate was normally calibrated before each run by the time-weight technique.

The establishment of the start-up configuration for the entire apparatus was performed just prior to ignition. The stripchart recorders, which were all set to run at 4 inches per minute, were synchronized. The propellant air and fuel and liquid tank pressures were set to the desired values. The propellant afr and fuel were then bled up to the main control values. The flow rates desired at ignition were established by opening one valve at a time and setting the desired flow rate of efther fuel, primary afr or secondary air. Five seconds prior to ignition all of the stripchart 
recorders were startad and ran for the duration of the experiment.

The start-up of the gas generator was aclomplished with the aforementioned automatic start sequencer.

\section{C.2 In-Run Procedure}

After mainstage ignition was secured, the operator brought the gas generator up to the nominal operating point. A period of time from five to ten minutes was required for the apparatus to reach thermal equilibrium after the operating point had been reached. During the warm-up period the film-coolant flow rate was started and the heat exchanger for preheating the liquid was turned on. The film of liquid on the test plate was visually inspected to ensure proper covarage. After thermal equilibrium was established and the desired operating point was set, the experimental measurements were begun. The mass transfer measurements followed the procedure outlined in Section 2.5 and Appendix B.4. The following tasks were performed during each mass transfer measurement: (a) three photographs of the liquid film were taken (if the camera was employed during the test), (b) the static pressure in the approach section to the test section (that was displayed on the precision Bourdon tube gage in the controi room) was recorded, (c) the readings on the 5 mercury manometers for the static pressure taps in the aluminum wedge were recorded (if applicable), and (d) the inlet and outlet temperatures for 
273

the liquid, that were displayed on their respective stripchart recorders, were noted and the reostat for controlling the inlet temperature was adjusted accordingly.

C.3 Post-Run Procedure

Upon the completion of an experimental test the gas generator was shut down. An afterbleed of propellant air was allowed to flow through the gas generator and test section to reduce the temperature of the equipment. The electric heat exchanger for preheating the film coolant was turned off. The liquid continued to flow onto the test plate during the cool-down period. 


\section{APPENDIX D}

\section{PHYSICAL PROPERTIES OF FILM COOLANTS}

\section{D.1 Subject Investigation}

Figures D.1-D.4 present the surface tension $\sigma$, the liquid viscosity $\mu_{1}$, the heat of vaporization $H_{v}$, and the vapor pressure $p_{v}$ as a function of the liquid temperature $T_{1}$. A separate plot is given for each of the film coolants of the subject investigation, namely, methanol, butanol, water, and RP-1. Because the film coolant RP-1 is a mixture of complex hydrocarbons, the properties for this coolant can be specified only approximately. The property values that are given herein for RP-1 assume, where necessary, that none of the volatiles have been removed. The circle that is presented for each curve in each of the figures represents the maximum withdrawal liquid temperature that was measured for that coolant during the course of the investigation. Each of the curves that is presented in Figs. D.1-D.4 was obtained from one or more of the references (56-60).

The molecular "wigitis of the subject fiim cuviants (employed in the calculation of the maximum liquid temperature $T_{1, m^{--}}$see Subsection 3.4 .1$)$ are as follows: methanol : 32 ; butanol : 74 ; water : $18 ;$ RP-1: 172 . 


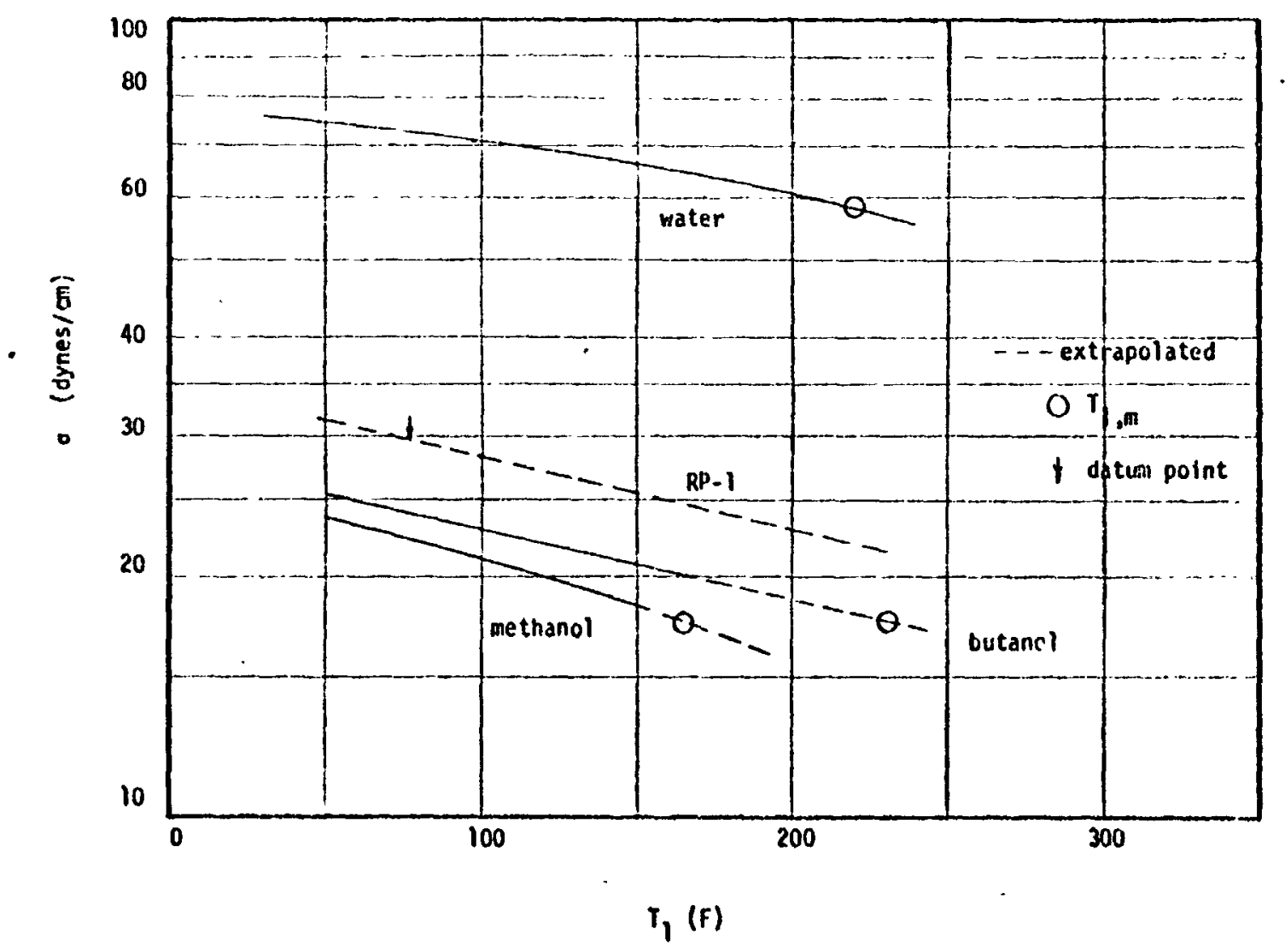

Fig. D.1. Surface Tension as a Function of Liquid Temperature for Liquids of Present Investigation 


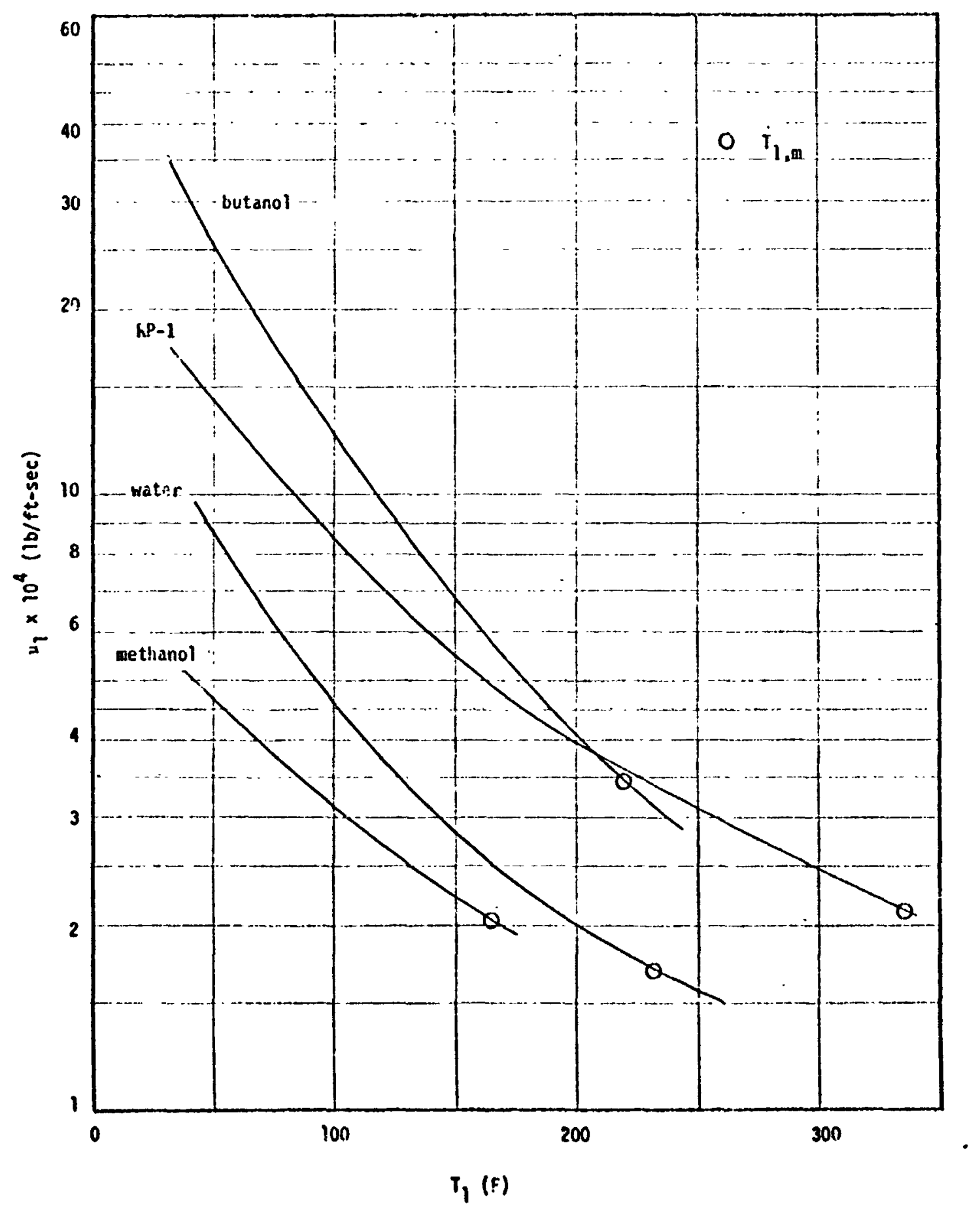

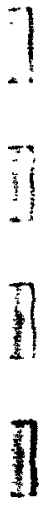

Fig. D.2. Liquid Viscosity as a Function of Liquid Temperature for Liquids of Present Investigation 


\section{7}

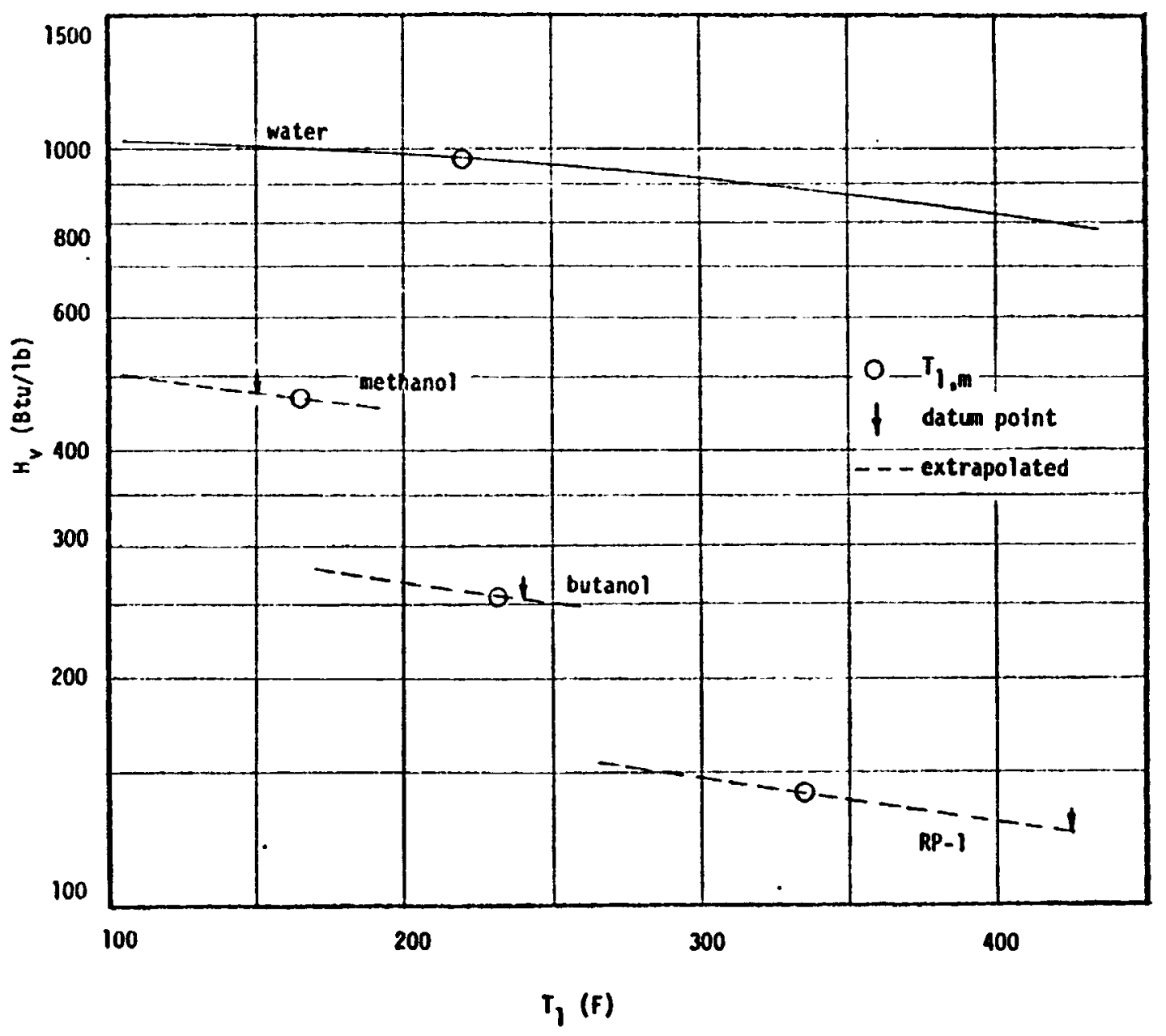

Fig. D.3. Heat of Vaporization as a Function of Liquid Temperature for Liquids of Present Investigation 


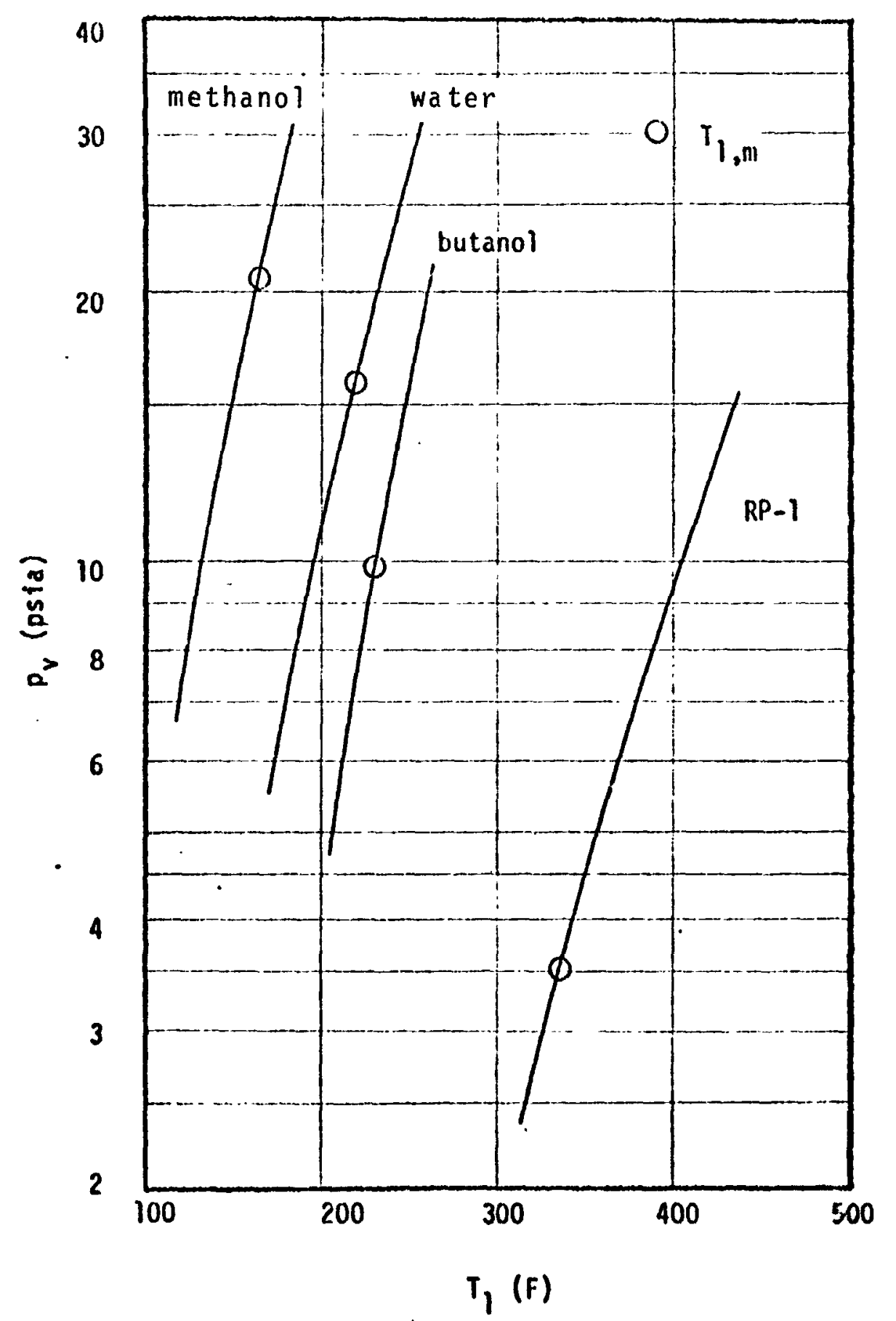

Fig. D.4. Vapor Pressure as a Function of Liquid Temperature for Liquids of Present Inves iligation 


\section{D.2 Investigation Due to Enmons}

Figures D.5-D.7 present the surface tension $\sigma$, the heat of vaporization $H_{v}$, and the vapor pressure $p_{v}$ as a function of the liquid temperature $T_{1}$ for each of the film coolants that was employed by Emmons (20), namely, ethanol, water, ammonia, and Freon-113. The values for the calculated maximum liquid temperature, $T_{1, m}$, and for the liquid reference temperature, $T_{1, r}$, are indicated for the coolants in each of the figures. Figure D.5 shows considerable extrapolation of the data for the surface tension $\sigma$. The extrapolation of the data for $\sigma$ was guided by the critical temperatures for the coolants; i.e., at the critical temperature the surface terision for the liquid is zero. The values for the critical temperature for the various coolants is given in Fig. D.5. Each of the solid curves that is presented in Figs. D.5-D.7 was obtained fromi one or more of the references $(56,58-61)$. 


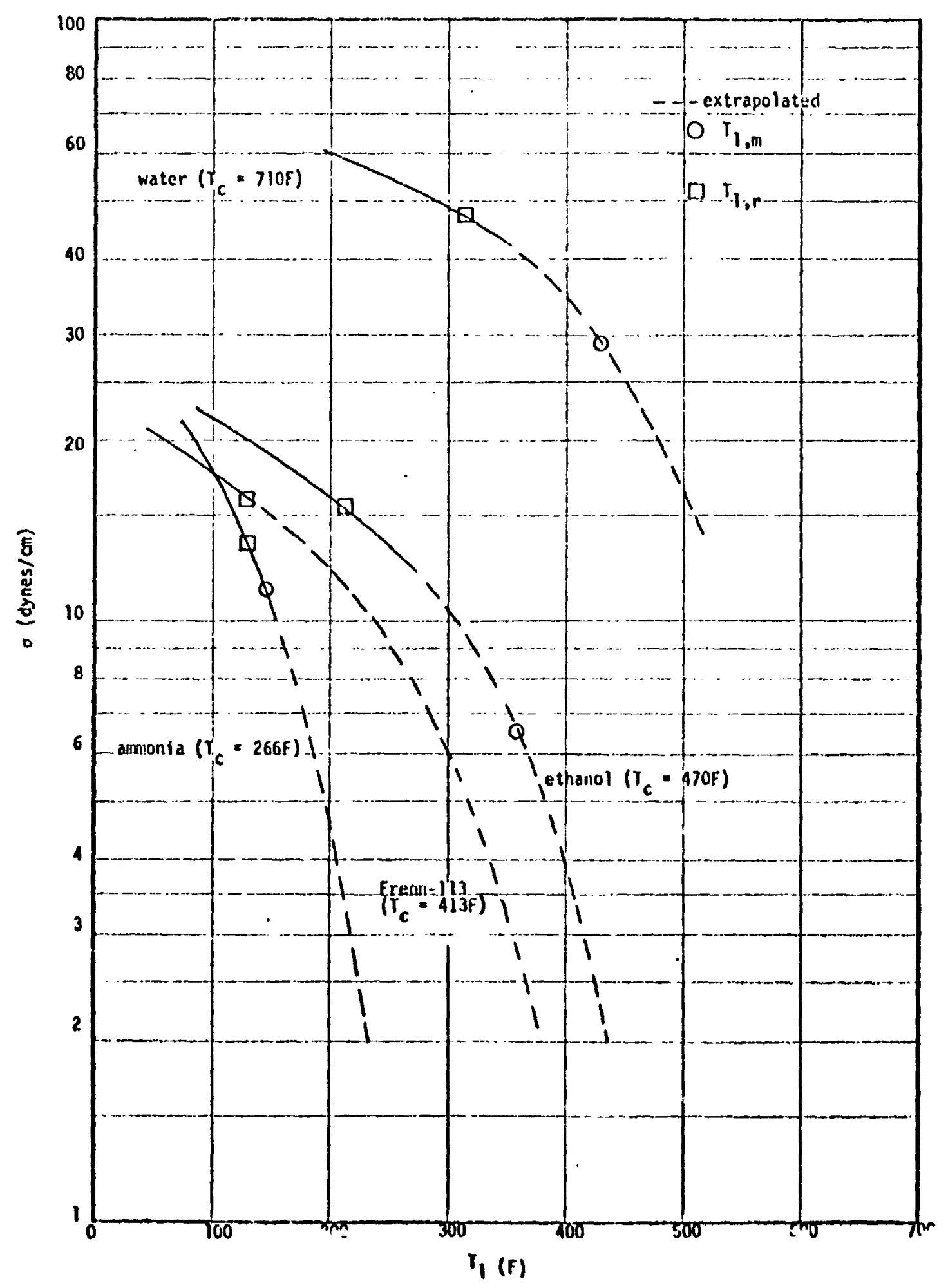

Fig. D.5. Surface Tension as a Function of Liquid Temperature for the Liquids Employed by Emmons 


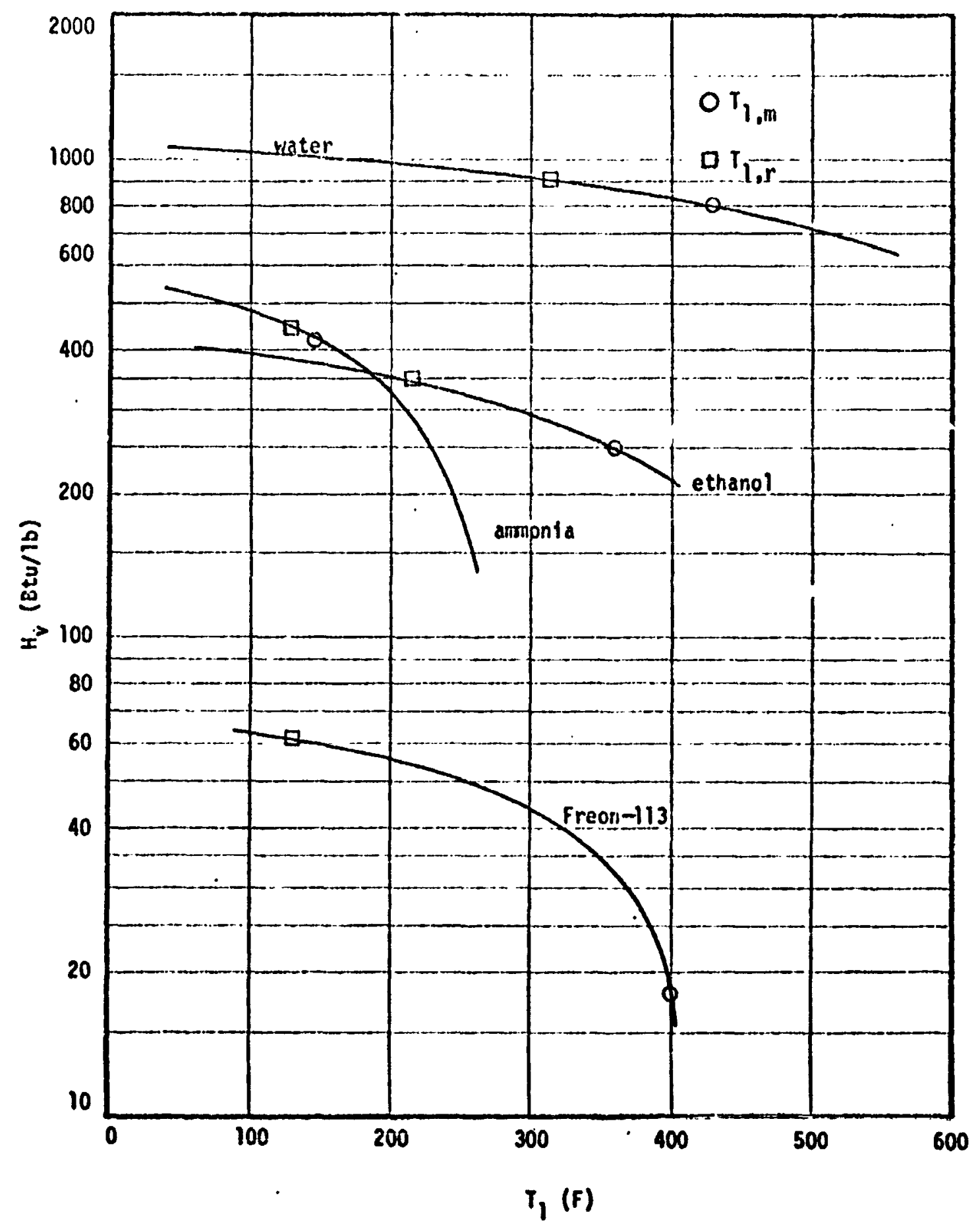

Fig. D.6. Heat of Vaporization as a Function of Liquid Temperature for the Liquids Employed by Emmons 


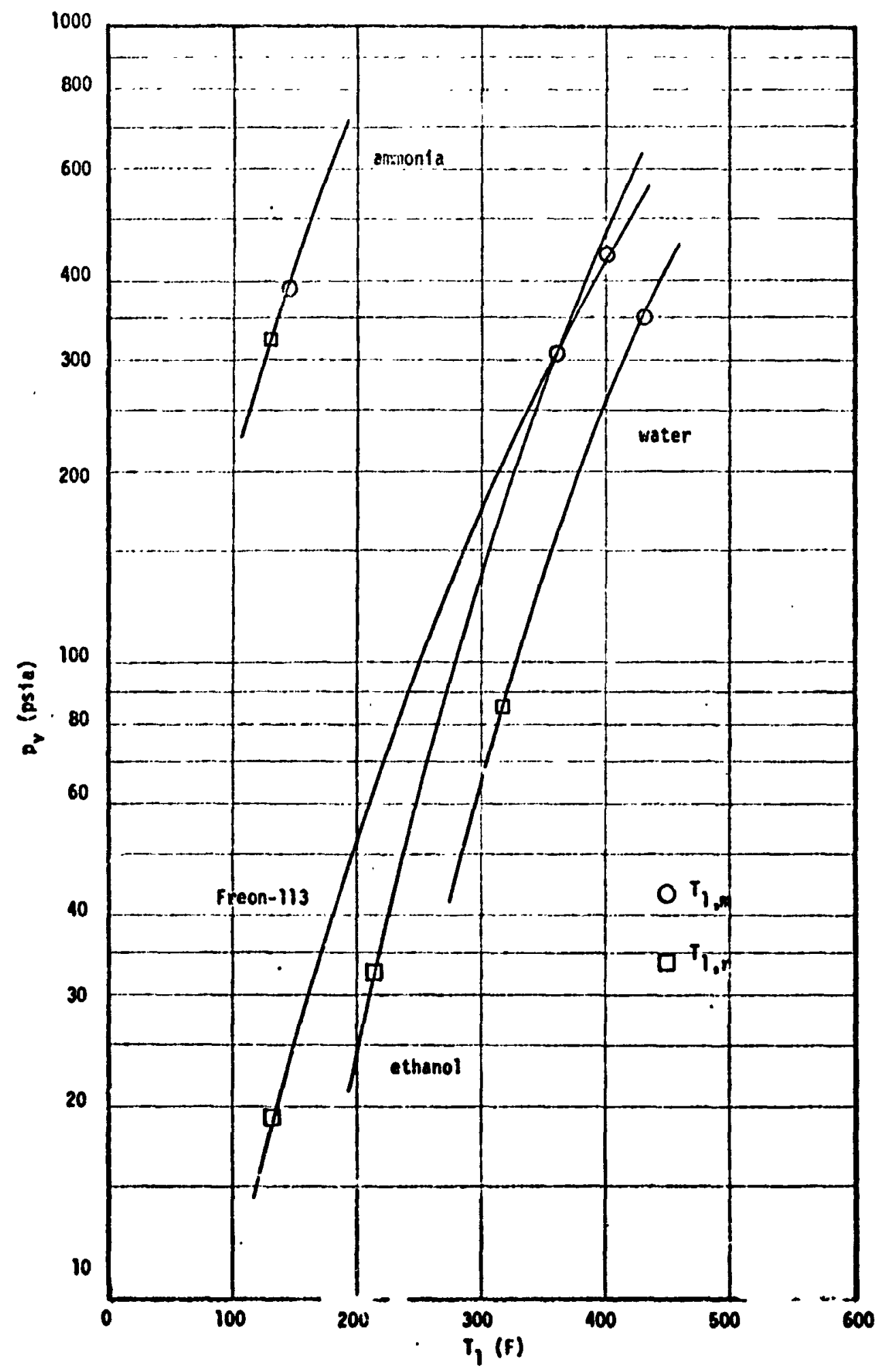




\section{APPENDIX E}

\section{A SAMPLE CALCULATION FOR THE MAXIMUM LIQUID TEMPERATURE}

To illustrate the manner in which the calculated results presented in Fig. 34 and in Fig. 35 were obtained, a sample calculation for the maximum liquid temperature $T_{1}, m$ for an air-water system (Fig. 34) is presented below.

For the flow conditions represented by Fig. 34 , Eq. (3-76b) reduces to the form

$$
\begin{aligned}
\frac{H_{v, m}}{c_{p_{a i r}}\left(T_{g}-T_{1, m}\right)} & =\frac{M_{g}}{M_{v}} \frac{p-p_{v, s}}{p_{v, s}} \\
& =1.61 \frac{p-p_{v, s}}{p_{v, s}}
\end{aligned}
$$

Solving Eq. (E-1) for the pa:tial pressure of the vapor at the interface, one obtains

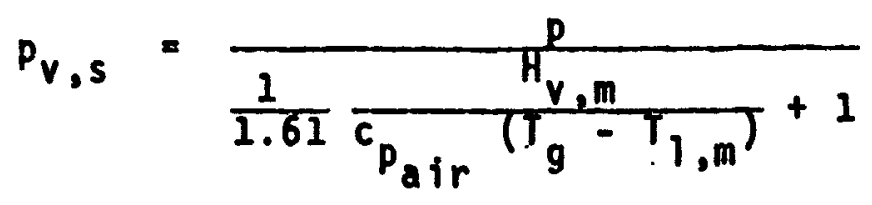


For purposes of illustration, assume that

$$
\begin{aligned}
T_{g} & =2000 R\left(c_{p_{a i r}}=0.2772 B t u / / b-R\right) \\
p & =500 \text { psia }
\end{aligned}
$$

For that case, Eq. $(E-2)$ reduces to

$$
p_{v, s}=\frac{500}{\frac{2.24 H_{v, m}}{\left(2000-T_{1, m}\right)}+1} \text { psia }
$$

To initiate the trial-and-error calculations, assume that $T_{1, m}=400 F=860 R$ (the accuracy of the first approximation for $T_{l, m}$ is not critical). For $T_{l, m}=960 R$, the data in Ref. 41 gives $H_{v, m}=826.0 \mathrm{Btu} / 1 \mathrm{~b}$ and $\mathrm{p}_{v, s}=247.3 \mathrm{psia}$. Substituting those data into Eq. (E-3) one obtains

$$
247.3 \text { psia } ? \frac{500}{1.624+1} \text { psia }
$$

$$
\text { f } 190.5 \text { psia }
$$

For the second calculation, assume that $p_{v, s}=190.5$ psia. Corresponding to $\mathrm{P}_{V, s}=190.5 \mathrm{ps} 1 \mathrm{a}$, the data in Ref. 41 gives $H_{v, m}=846.7 \mathrm{Btu} / \mathrm{lb}$ and $T_{1, m}=837.6 R_{\text {. }}$ Substituting those data into Eq. (E-3) one obtains

$$
\begin{aligned}
290.5 \text { psia } & =\frac{500}{3.633+1} \text { psia } \\
& \neq 189.9 \text { psia }
\end{aligned}
$$


Continuing this procedure, we assume for the next calculation that $p_{V, S}=189.9 \mathrm{psia}$. Corresponding to $\mathrm{p}_{\mathrm{V}, \mathrm{s}}=189.9$ psia, the data in Ref. 41 gives $H_{v, m}=846.9 \mathrm{Btu} / 1 \mathrm{~b}$ and $T_{1, m}=377.4 F=837.4 R$. Substituting those data into Eq. $(E-3)$, one obtains

$$
\begin{aligned}
189.9 & \stackrel{?}{=} \frac{500}{1.633+1} \text { psia } \\
& =189.9 \text { psia }
\end{aligned}
$$

Thus, for $T_{1, m}=2000 R$ and $p=500 \mathrm{psia}$, and for the flow conditions represented by Fig. 34 , the calculated value of the maximum liquid temperature is $\mathrm{T}_{1, \mathrm{~m}}=377.4 \mathrm{~F}=837.4 \mathrm{R}$. 


\section{F.1 General Data Reduction}

The pressure transducers that were employed to measure the static and total pressures in the gas generator system and the test secticn were calibrated against a precision pressure gage prior to each experiment. The calibration was in the form of discrete pressure steps both ascending and descending; the maximum value of the largest calibration step was in excess of the value recorded during an experiment. The calibration and the corresponding run data were both recorded on the same Brown stripchart recorder. The numerical value of the particular pressure parameter recorded during an experiment was determined by linear interpolation of the recorded transducer output against the calibration. The estimated error resulting from the transducer hysteresis and data transcription was estimated at \pm 2 percent at the nominal run value of pressure.

All of the temperatures monitored during an experimant were recorded either on direct reading Brown recorders or on pre-calibrated Brown recorders. The values of tempera- . ture recorded during an experiment were correspondingly 
read off directly from the recorder chart or determined with aid of a calibration curve. The estimated error in the temperatures read from the Brown recorders was from \pm 2 to \pm 4 percent at the nominal run value of the temperature.

The volume flow rates of air, fuel and film coolant were measured with turbine flowmeters. The flowmeters which ineasured the flow rates of the fuel and film coolant were calibrated with the respective liquid in terms of frequency output as a function of weight flow rate. In this manner a calibration constant was established for a particular flowmeter through which the weight flow rate and frequency output were related. The flowmeter employed to meter the flow rate of air to the gas generator was calibrated by the company supplying the instrument as volume rate of flow versus frequency at a static pressure of $600 \mathrm{psig}$. The flowmeter was subsequently calibrated again at the Jet Propulsion Center, Purdue University, against ASME standard orifices at flow pressures of 400,600 , and $800 \mathrm{psig}$. The latter calibration and the calibration supplied with the meter agreed within \pm 5 percent at the nominal run value. The weight flow rate of air measured during a run was evaluated as

$$
W=Q \rho_{a}
$$


where

$Q$ = indicated volume flow rate at the frequency output of the flowmeter

$\rho_{a}=$ density of the air determined from the measured static pressure and temperature of the air immediately upstream of the flowmeter. and employing the equation of state for a perfect gas

The output circuit of the flowmeters was calibrated prior to each experiment against known frequencies available in the flowmeter instrumentation. The estimated error in the measurement of the film coolant flow rates was \pm 2 percent or less of the nominal run value. The estimated error in the measurement of the air flow rate was taken as the deviation of the two aforementioned calibrations, that is, \pm 5 percent.

\section{F.2 Gas Stream Parameters}

The procedure that was employed to compute the values for the gas stream parameters $G, u_{g}$, and $M_{0}$ at the leading edge of the experimental test plate is outlined as follows: (a) A first estimate for the gas mass velocity, G, was obtained by assuming one-dimensional flow in the experimental tunnel, in which case it follows from the conservation of mass that (neglecting the fuel flow rate into the gas generator)

$$
W=G A_{c r}
$$


where

$$
\begin{aligned}
& W=\text { measured air flow rate } \\
& A_{c r}=\text { cross-sectional area of the experimental tunnel }
\end{aligned}
$$

(b) The displacement thickness for the turbulent boundary layer on each side wall of the test section at the leading edge of the wetted test plate was computed from the classical expression (36)

$$
\delta_{1}^{*}=0.048 x_{1} \operatorname{Re}_{x_{1}}^{-0.2}
$$

where

$$
\begin{aligned}
\operatorname{Re}_{x_{1}} & =\frac{G x_{1}}{\mu_{g}} \\
\mu_{g} & =\begin{array}{l}
\text { viscosity for the gas stream evaluated at the } \\
\text { measured gas stream temperature }
\end{array} \\
x_{1} & =40 \text { inches }
\end{aligned}
$$

(c) A corrected cross-sectional area for the flow at $x=x_{1}$, denoted by $A_{C r}^{\prime}$, was computed from

$$
A_{c r}^{\prime}=A_{c r}-\delta_{1}^{*} \cdot(\text { wetted perimeter of tunnel) }
$$

(d) The final value for the gas mass velocity was computed by multiplying the result of step (a) by the ratio of the uncorrected to the corrected cross-sectional area for the tunnel at $x=x_{1}$.

(e) The gas stream velocity, $u_{g}$, was computed from the . result of step (d) for the mass velocity $G$ together with 
the expression

$$
u_{g}=\frac{G}{\rho_{g}}
$$

where the gas stream density $\rho_{g}$ was computed from the equation of state for a perfect gas and the measured values for the gas stream pressure and temperature.

(f) The momentum parameter, $M_{0}$, was computed from

$$
M_{0}=\rho_{g} u_{g}^{2}
$$

The accuracy of the computed values for the foregoing parameters is estimated as \pm 5 percent for $u_{g}$ and $G$, and \pm 5 to 10 percent for $M_{0}$.

For those tests in which the gas stream was not accelerated over the wetted test plate, the values for the parameters $u_{g}, G$, and $M_{0}$ were assumed constant throughout the test section. For those tests where the gas stream was accelerated over the wetted test plate, the local values for the gas stream parameters $u_{g}, G$, and $M_{0}$ for $x>x_{l}$ were computed from the static pressure measurements that were made down the length of the test section. Those calculations and measurement: l:ere discusseu previole'y ir. Subsection 3.3 .1 and will not be repeated here. The accuracy of the values that were computed for those local flow parameters is estimated as \pm 5 percent for $u_{g}$ and $G$, and . 
\pm 5 to 10 percent for $M_{0}$.

\section{F.3 Mass Transfer Data}

The procedure that was employed to measure the net rate of mass transfer from the liquid film to the gas stream has been described in detail in Section 2.5. Moreover, the operation of the senarator that was utilized to measure the rate of liquid withdrawal from the wetted test plate has been. adequately described in Appendix B.4.

During the course of any one experimental test, a number of consecutive measurements of the rate of liquid withdrawal was normally made for one or more fixed rates of liquid injection in order to check the reproducibility of the data. The measured rates of liquid withdrawal could always be reproduced with an accuracy of 5 percent or better.

A concerted effort was not made to try to reproduce the experimental data of any one experimental test by conducting an independent experimental test at the same flow conditions. It was very difficult to operate the hot gas generator at the same run point for two different experimental tests. The primary problem was that of establishing the same rate of air flow (and, therefore, gas mass velocity or gas stream Reynolds number) on two different occasions. However, a matching of the run conditions was realized for two different sets of experimental tests, and in each case the measurements of mass transfer were found to reproduce to within an accuracy of 10 percent or better. 
Table G.l lists the measured or computed values for the pertinent flow parameters for the experimental tests with a constant velocity gas stream. The letter that follows the test number indicates the film coolant for that test; i.e., $M$ - methanol; B - butanol; W-water; and $R$ - RP-1. The parameter values 1 isted in the table are the average of the values that were measured for each of the datum points for mass transfer during an experimental test. From 6 to 15 datum points were obtained for each test. Not listed in Table G.I are those experimental tests that were concerned only with photographic data, those tests which were purely exploratory as regards experimental technique, etc., or those tests for which some failure of the experimental apparatus voided the test.

Table G.2 presents a similar listing of the average values of the pertinent flow parameters for the experimental tests with an accelerating gas stream.

Figures G.I through G.13 present graphically the mass transfer data for the experimental tests listed in Table G.1. Figure G.14 presents the mass transfer data for the 


\section{3}

experimental tests that are listed in Table G.2. 


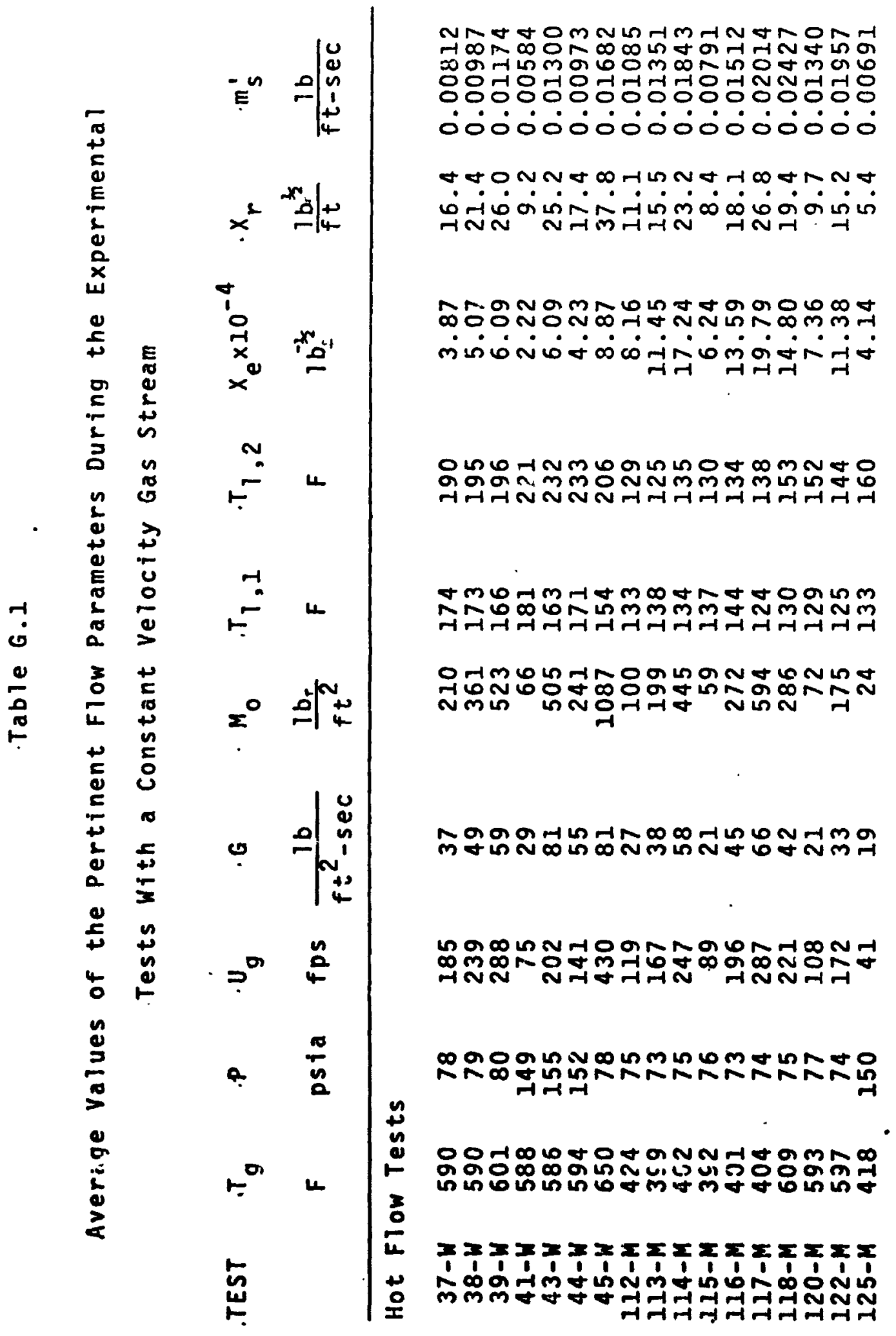

NNA

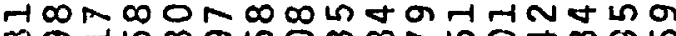

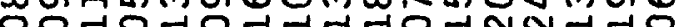

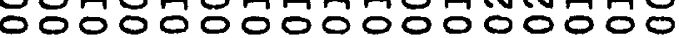
í $\dot{0} \dot{0} \dot{0} \dot{0} \dot{0} \dot{0} \dot{0} \dot{0} \dot{0} \dot{0} \dot{0}$

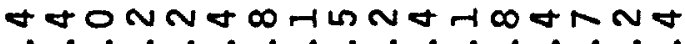
$\dot{0} \dot{0} \dot{0} \dot{0} \dot{0} \dot{0} \dot{0} \dot{0} \dot{0} \dot{0}$

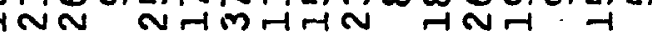

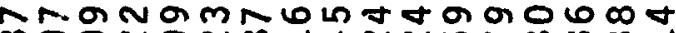

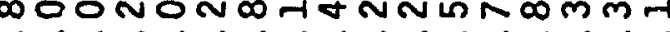

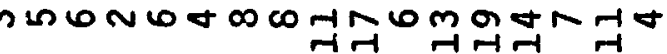

On日 HNm

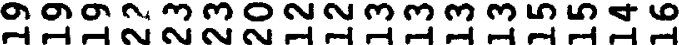

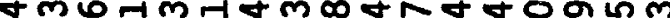
NDOWNUMmm

O HWN min un

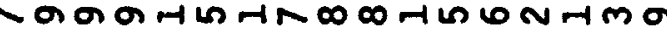

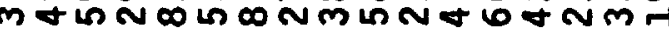

nक⿻

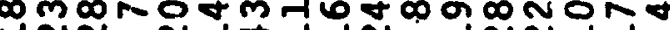

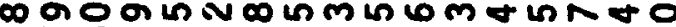
dinnRNRMRNRN

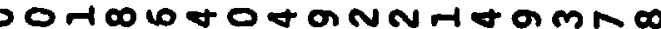
ผ

Tz: -

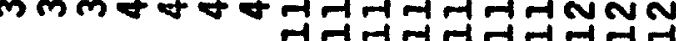




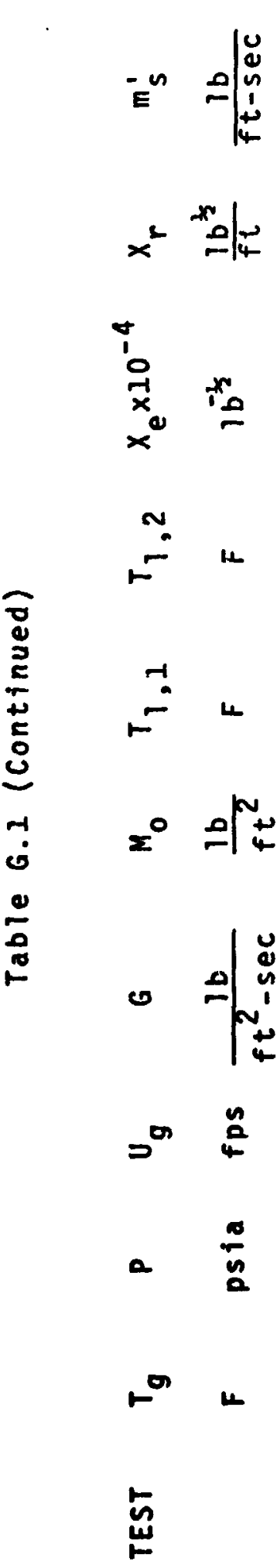

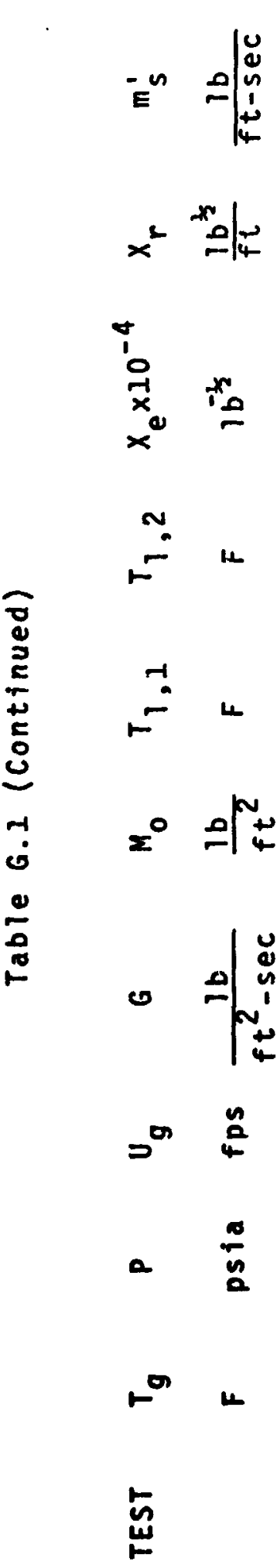

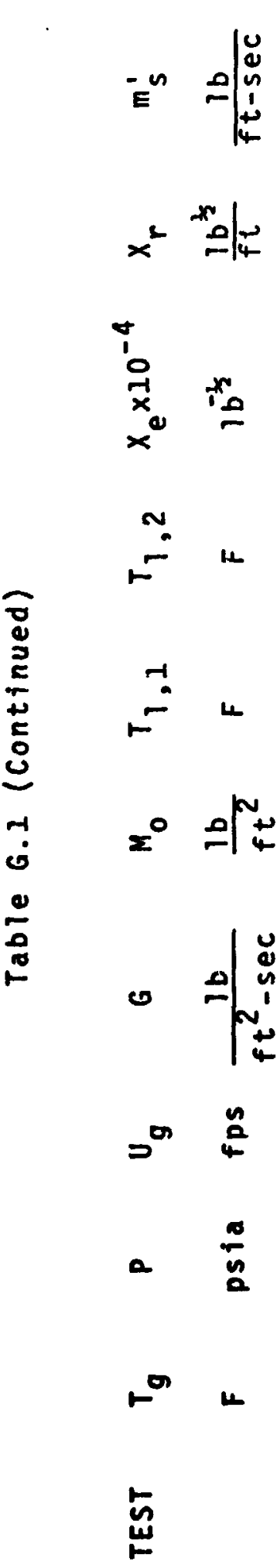

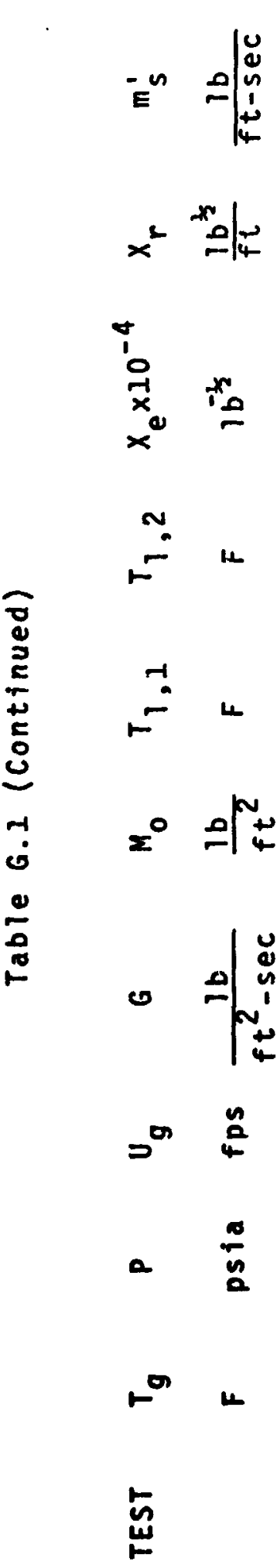

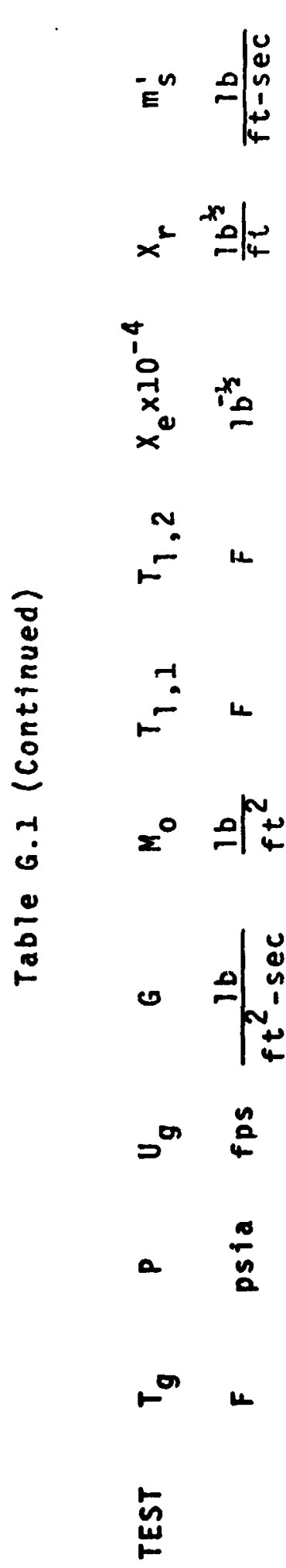

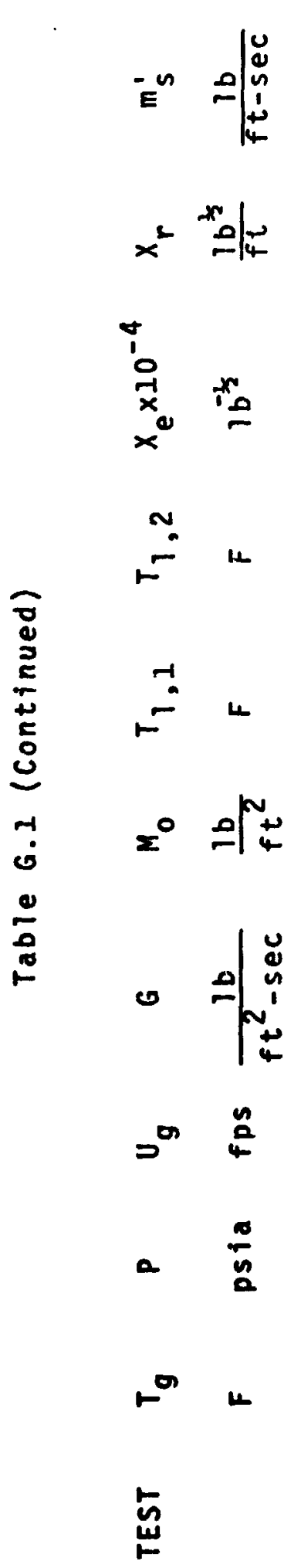

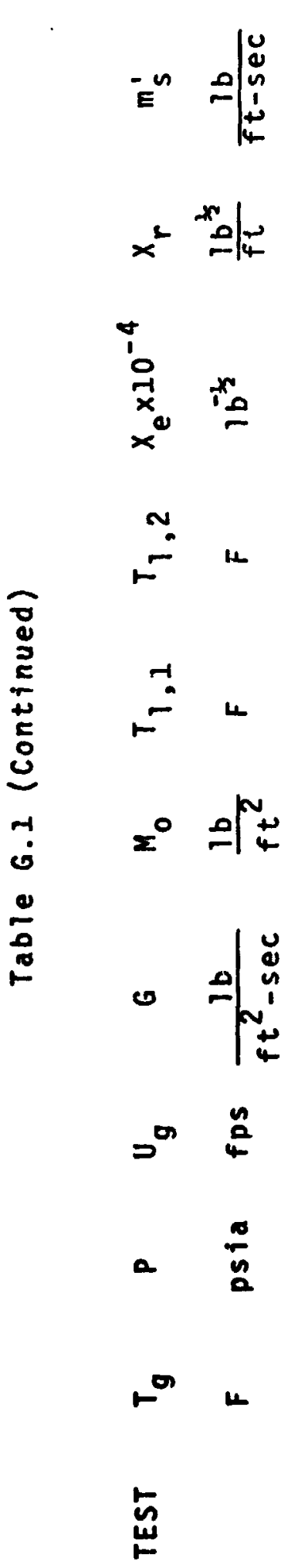

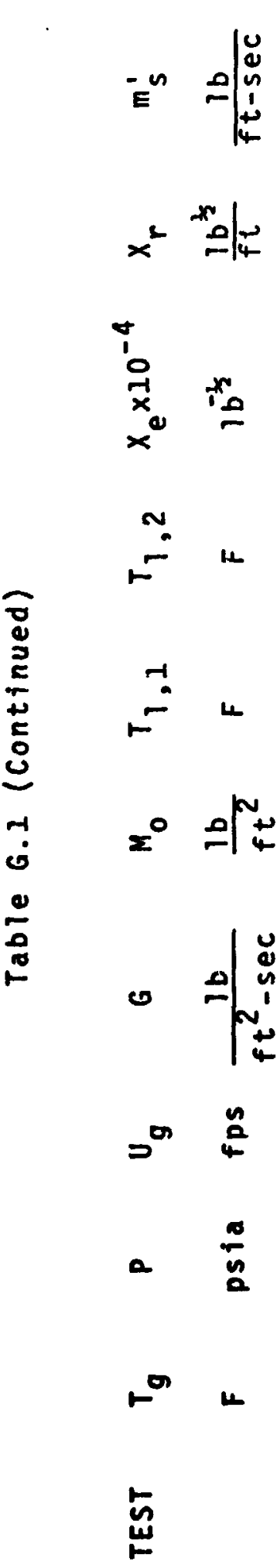

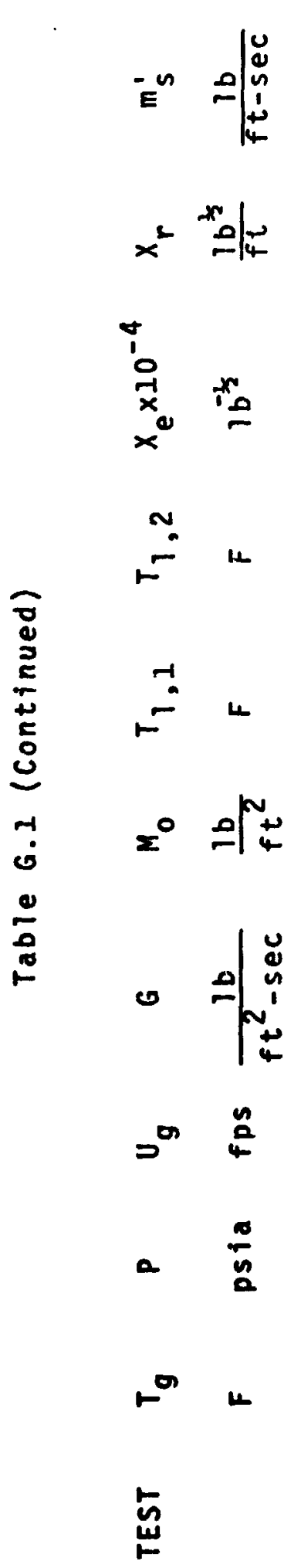

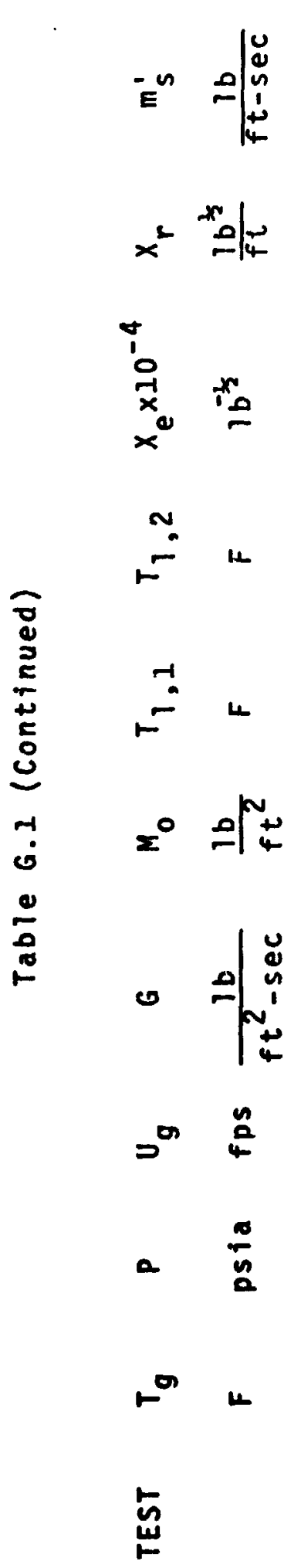

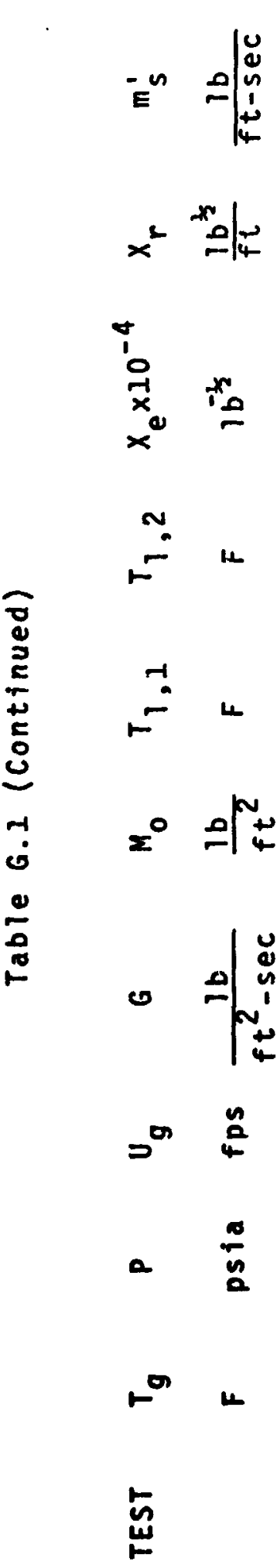

HLNUGNDON

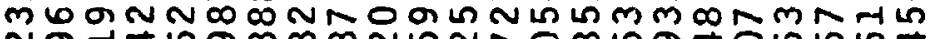
NaनUum

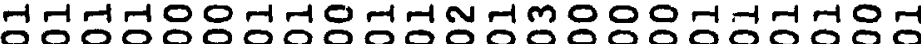
. i0000000000000000.j00000 V G

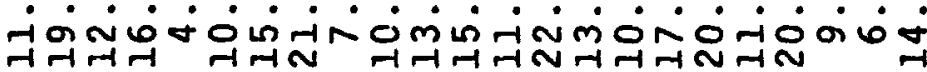

mNmNOLmG ᄉmm

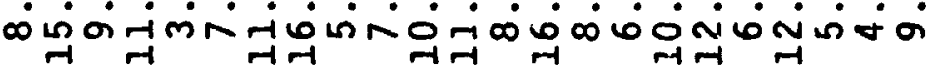

a d. OOA

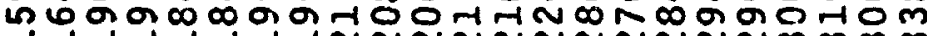
HAHAHAHMNNNNNNNNNNNMMMM m

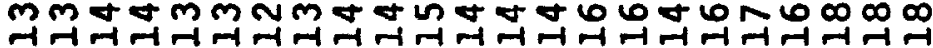

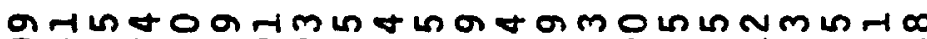

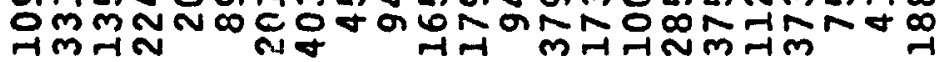

"1Nand

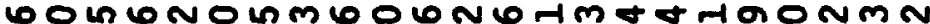

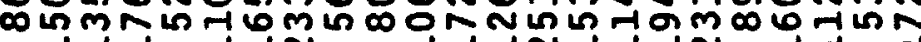

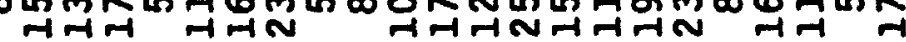

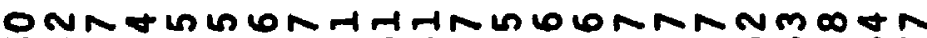

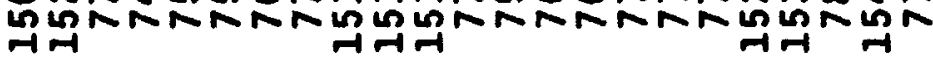

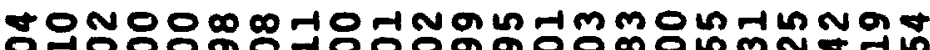

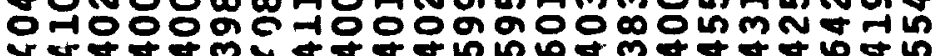
हर

I =

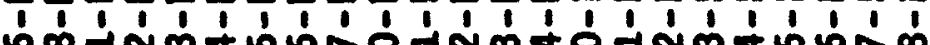

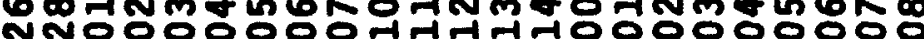

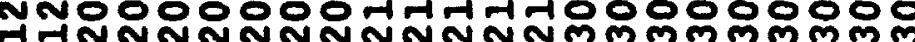




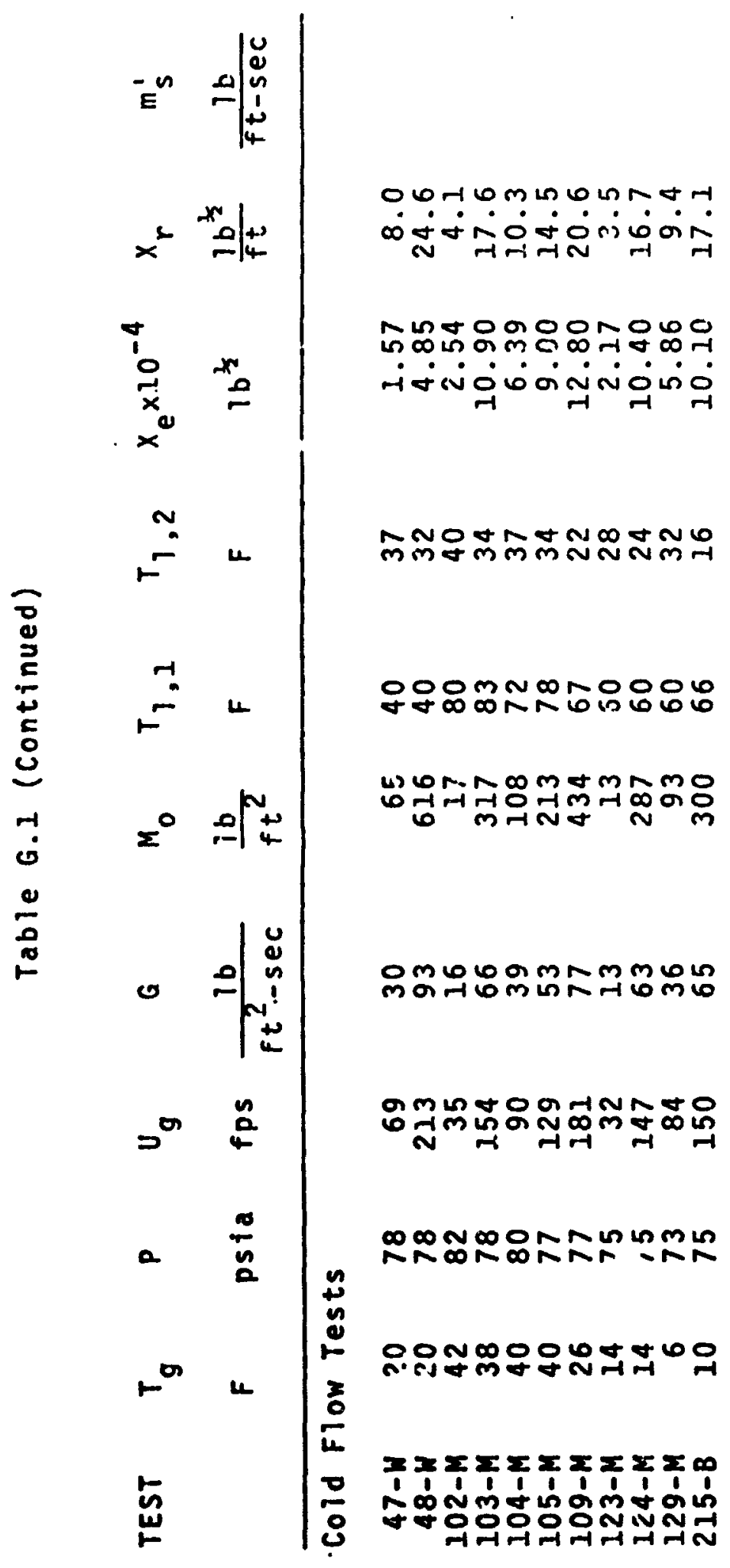


Table G.2

Average Values of the Pertinent Flow Parameters During the Experimental Tests With An Accelerating Gas Stream

\begin{tabular}{|c|c|c|c|c|c|}
\hline \multirow[t]{2}{*}{ Test } & $\mathrm{T}_{\mathrm{g}}^{\mathrm{o}}$ & $p^{0}$ & $T_{1,1}$ & $T_{1,2}$ & $m_{s}^{\prime}$ \\
\hline & $F$ & psia & $F$ & $F$ & $\frac{1 b}{f t-s e c}$ \\
\hline $\begin{array}{l}401-M \\
402-M \\
403-M \\
405-M \\
502-W \\
503-W \\
505-W \\
507-W\end{array}$ & $\begin{array}{l}442 \\
434 \\
440 \\
447 \\
444 \\
435 \\
405 \\
480\end{array}$ & $\begin{array}{r}74 \\
52 \\
103 \\
126 \\
103 \\
54 \\
99 \\
125\end{array}$ & $\begin{array}{r}139 \\
140 \\
144 \\
135 \\
118 \\
78 \\
78 \\
71\end{array}$ & $\begin{array}{l}142 \\
126 \\
154 \\
173 \\
195 \\
163 \\
188 \\
213\end{array}$ & $\begin{array}{l}0.0219 \\
0.0168 \\
0.0267 \\
0.0314 \\
0.0102 \\
0.0062 \\
0.0093 \\
0.0132\end{array}$ \\
\hline
\end{tabular}




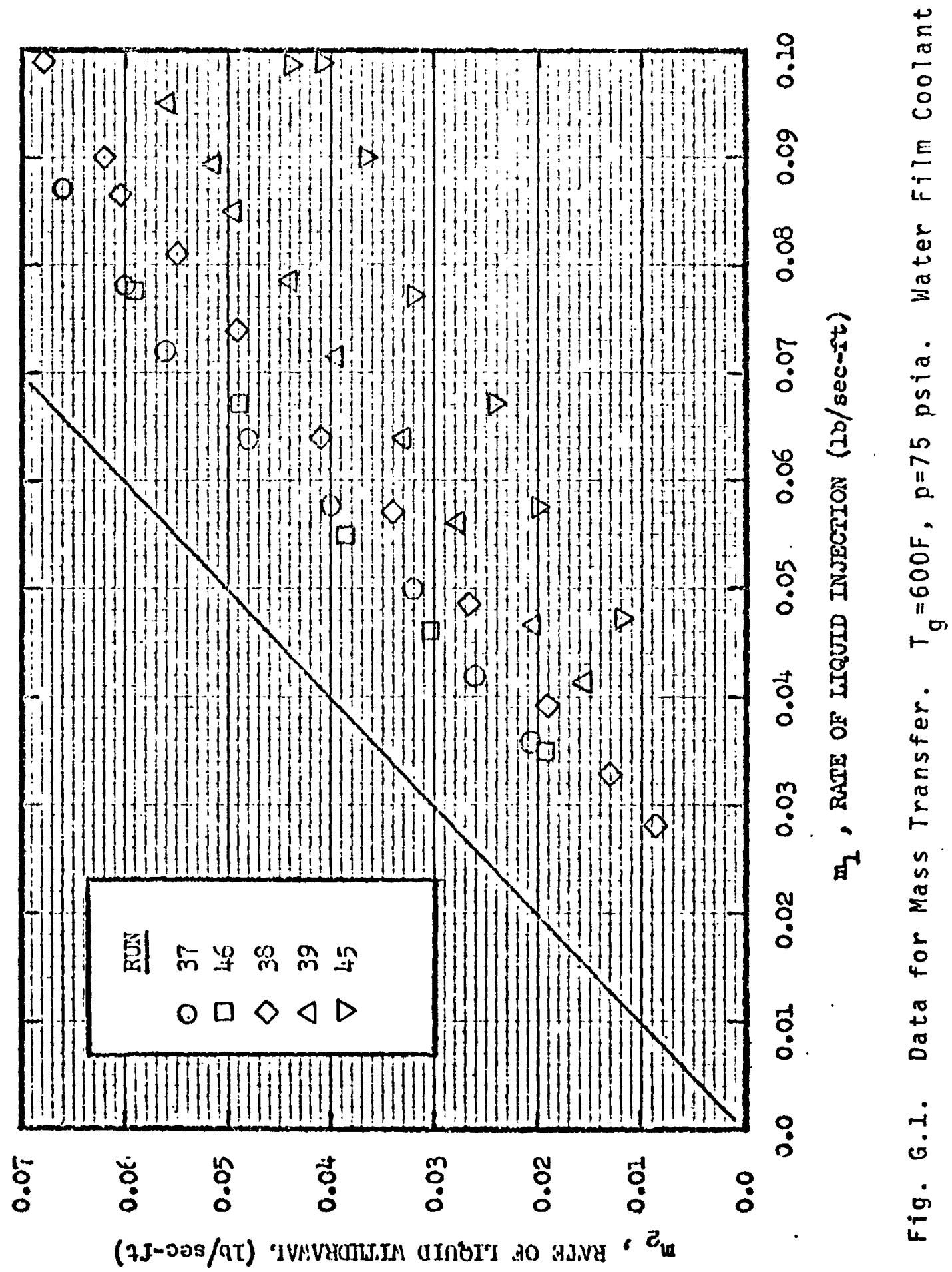



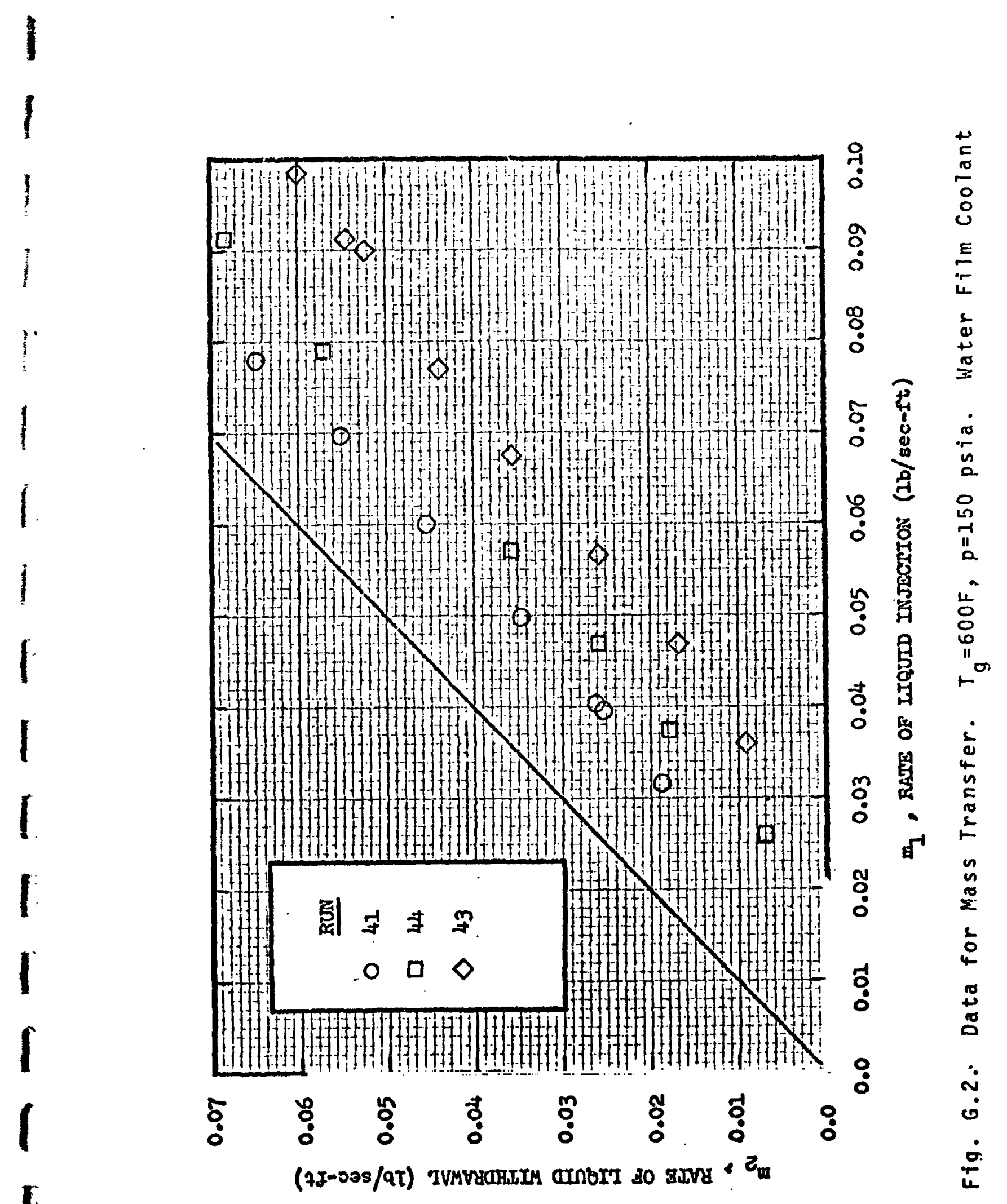

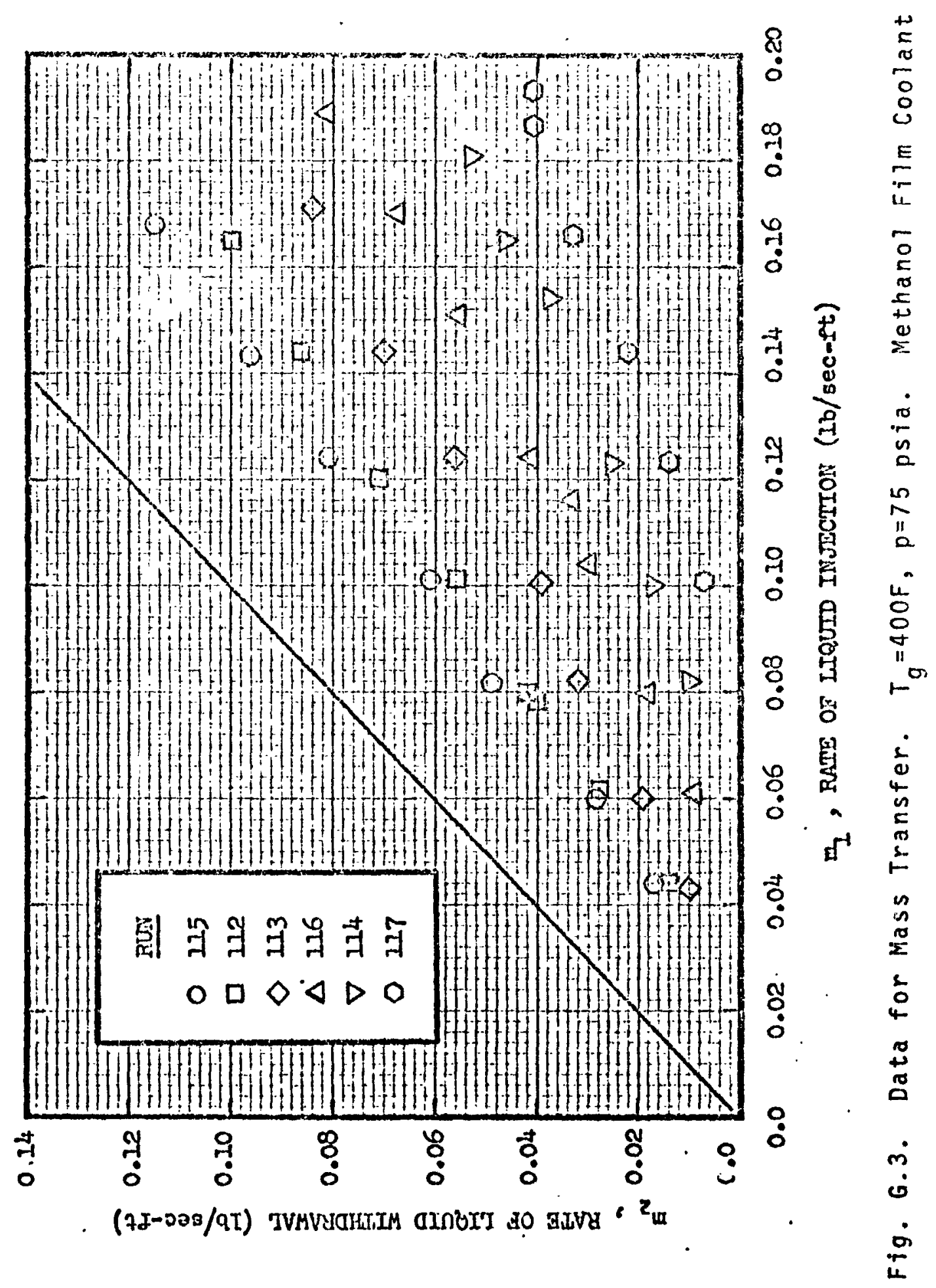


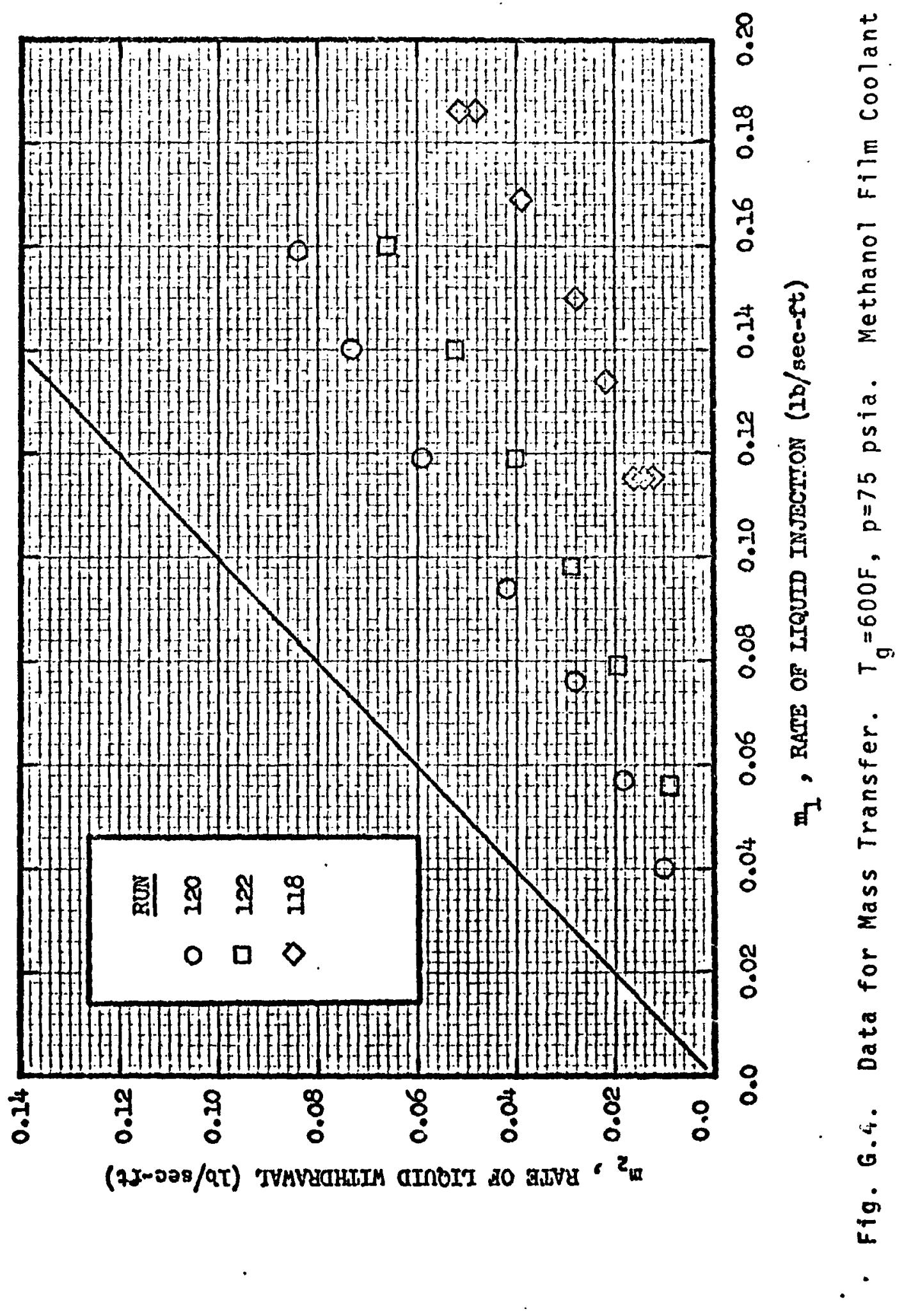




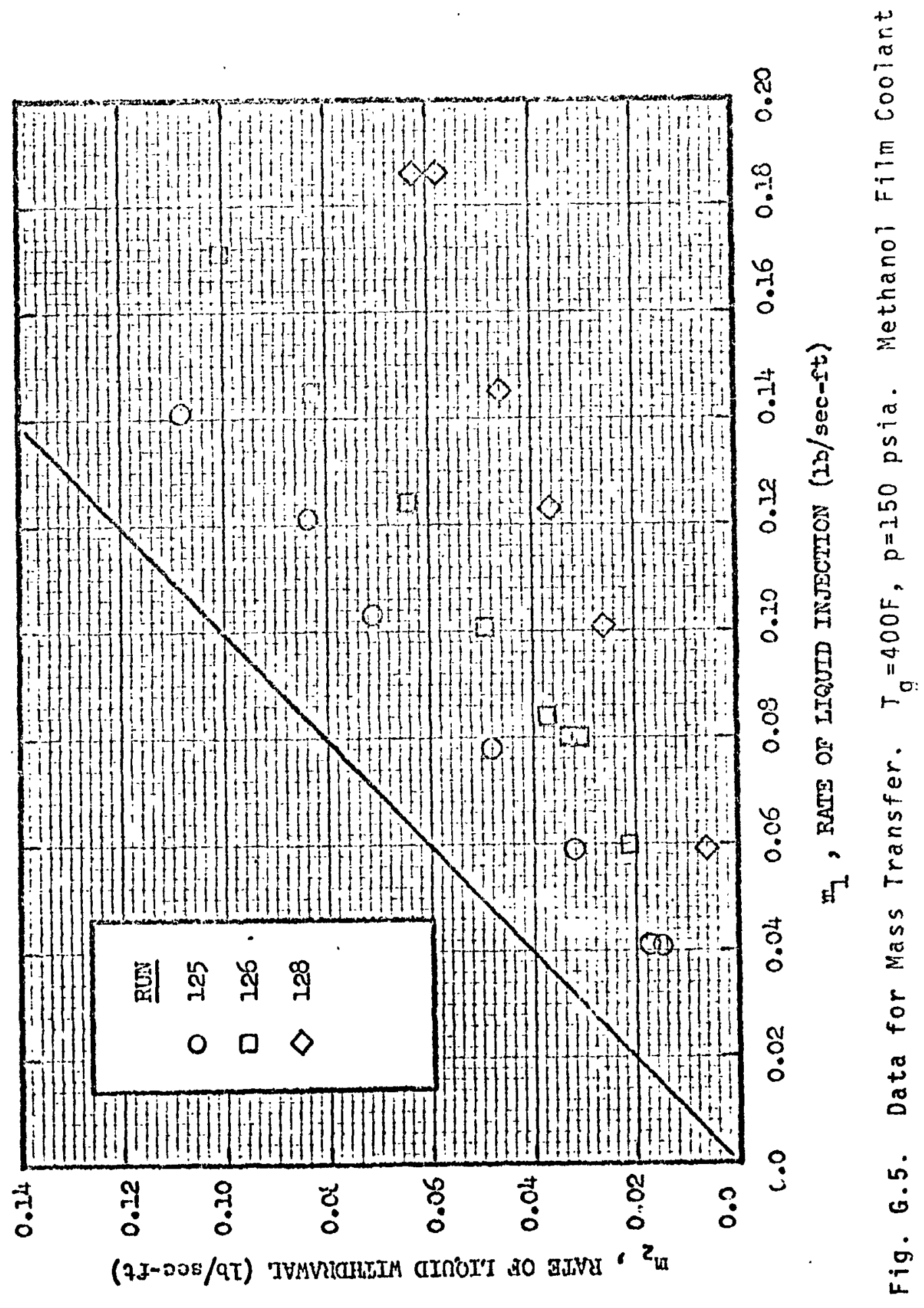



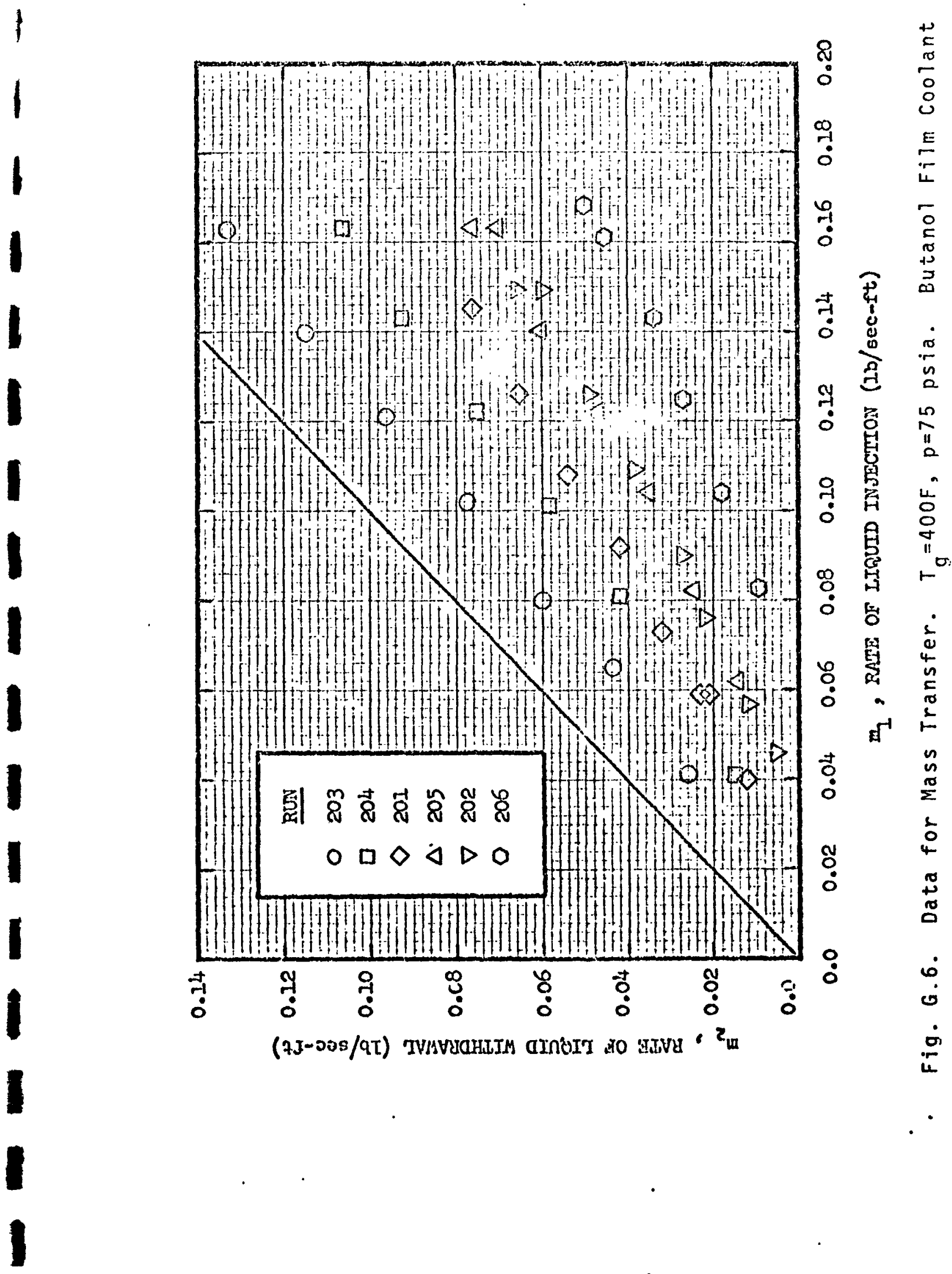


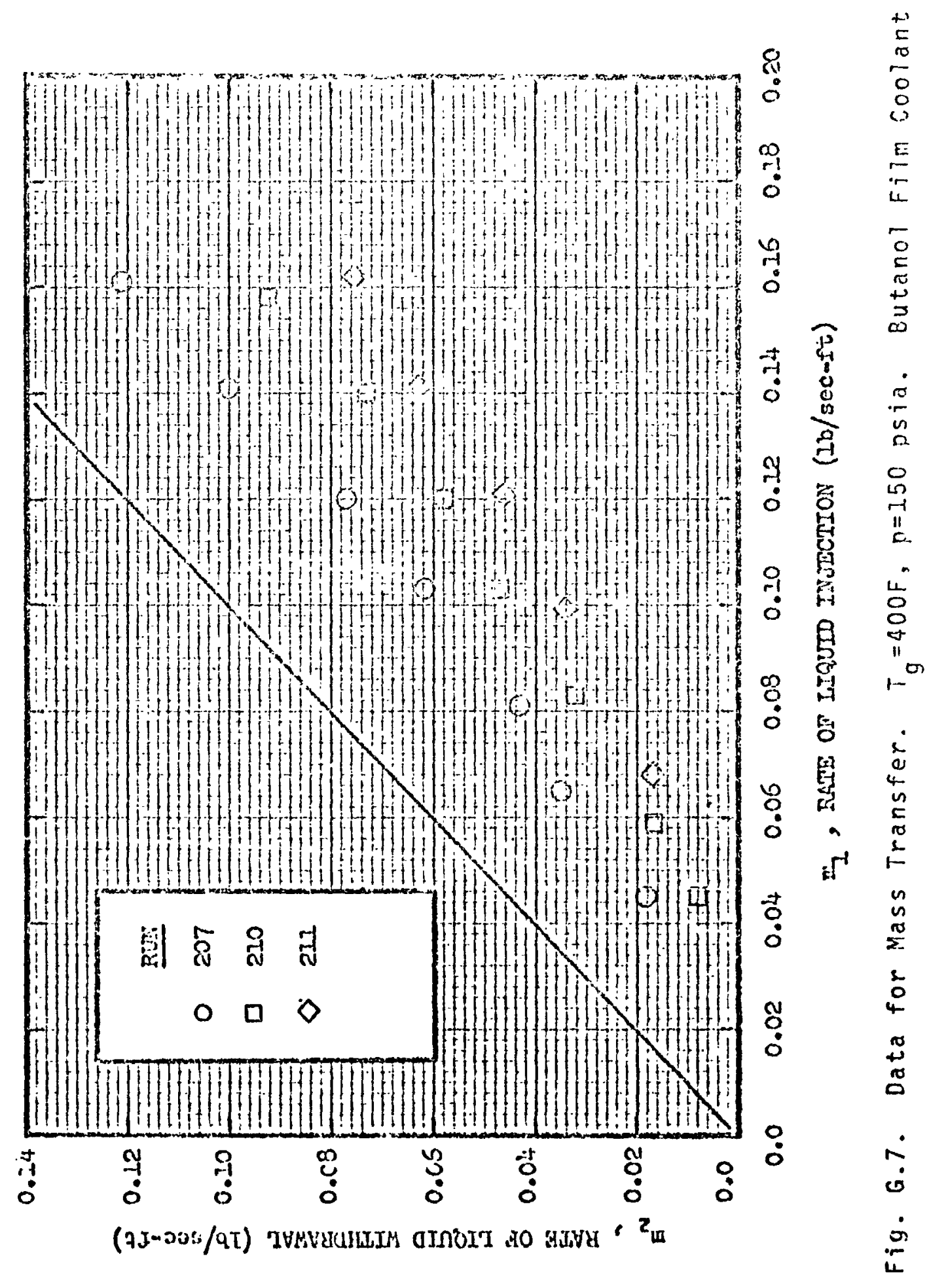




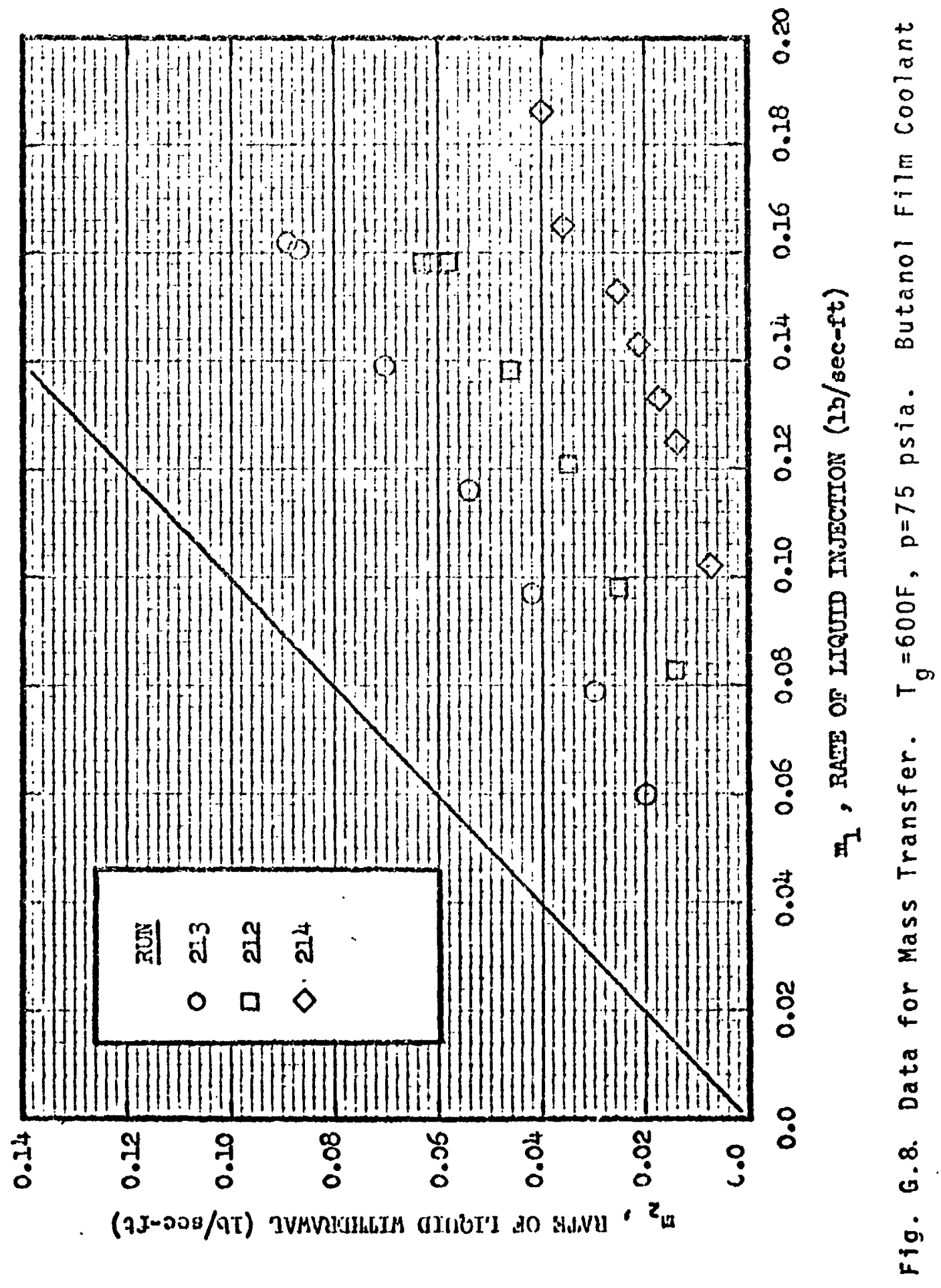




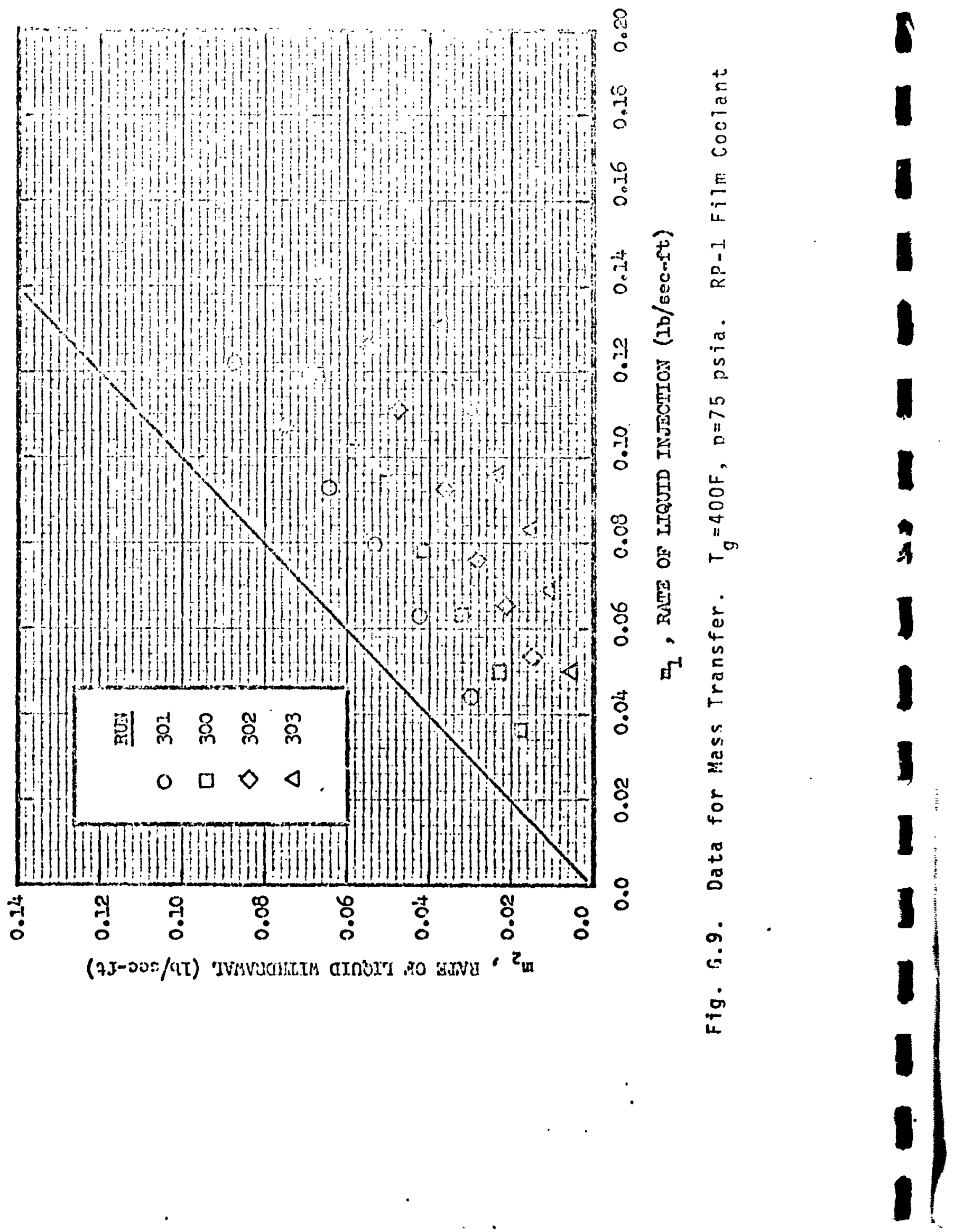




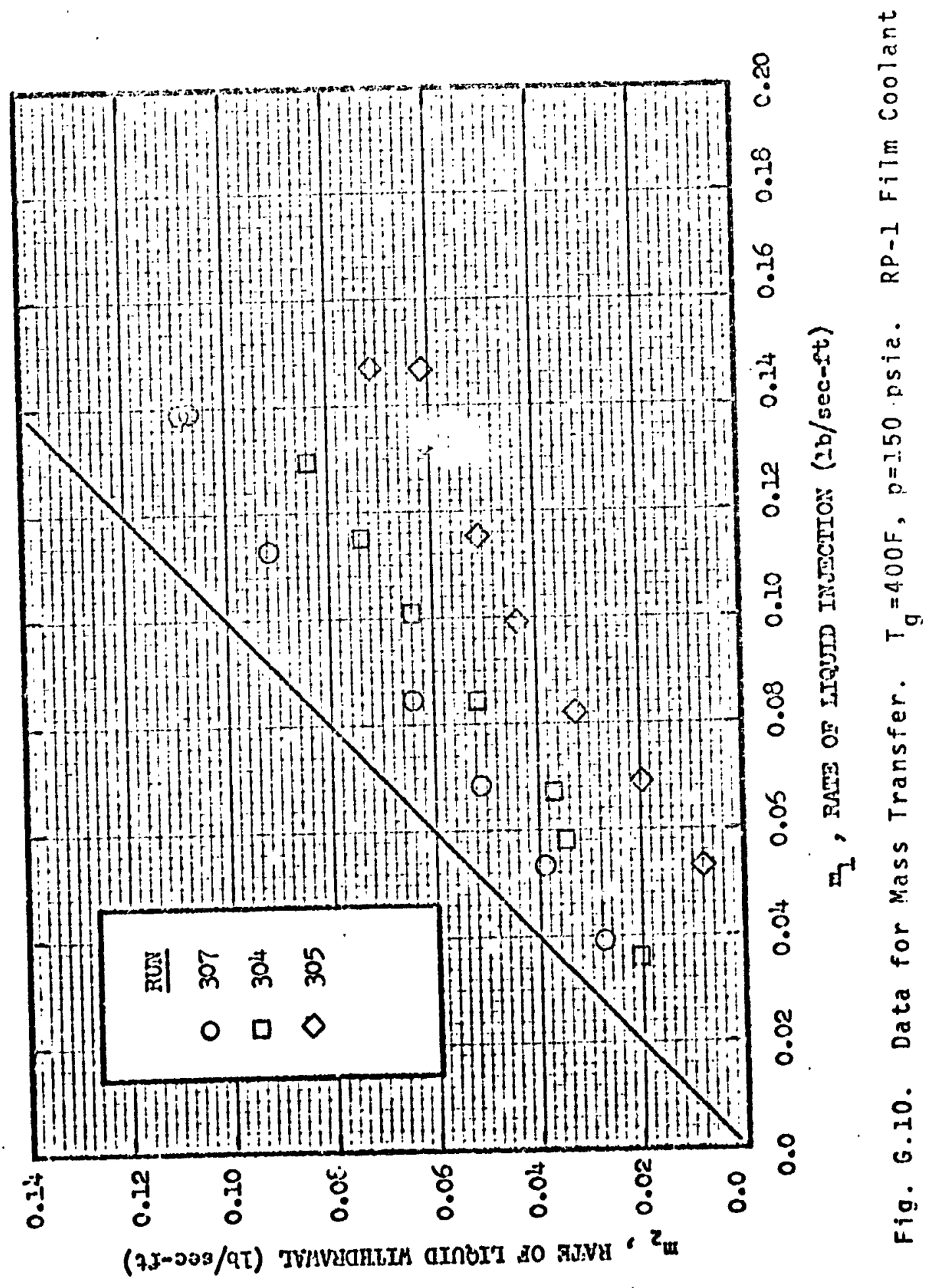




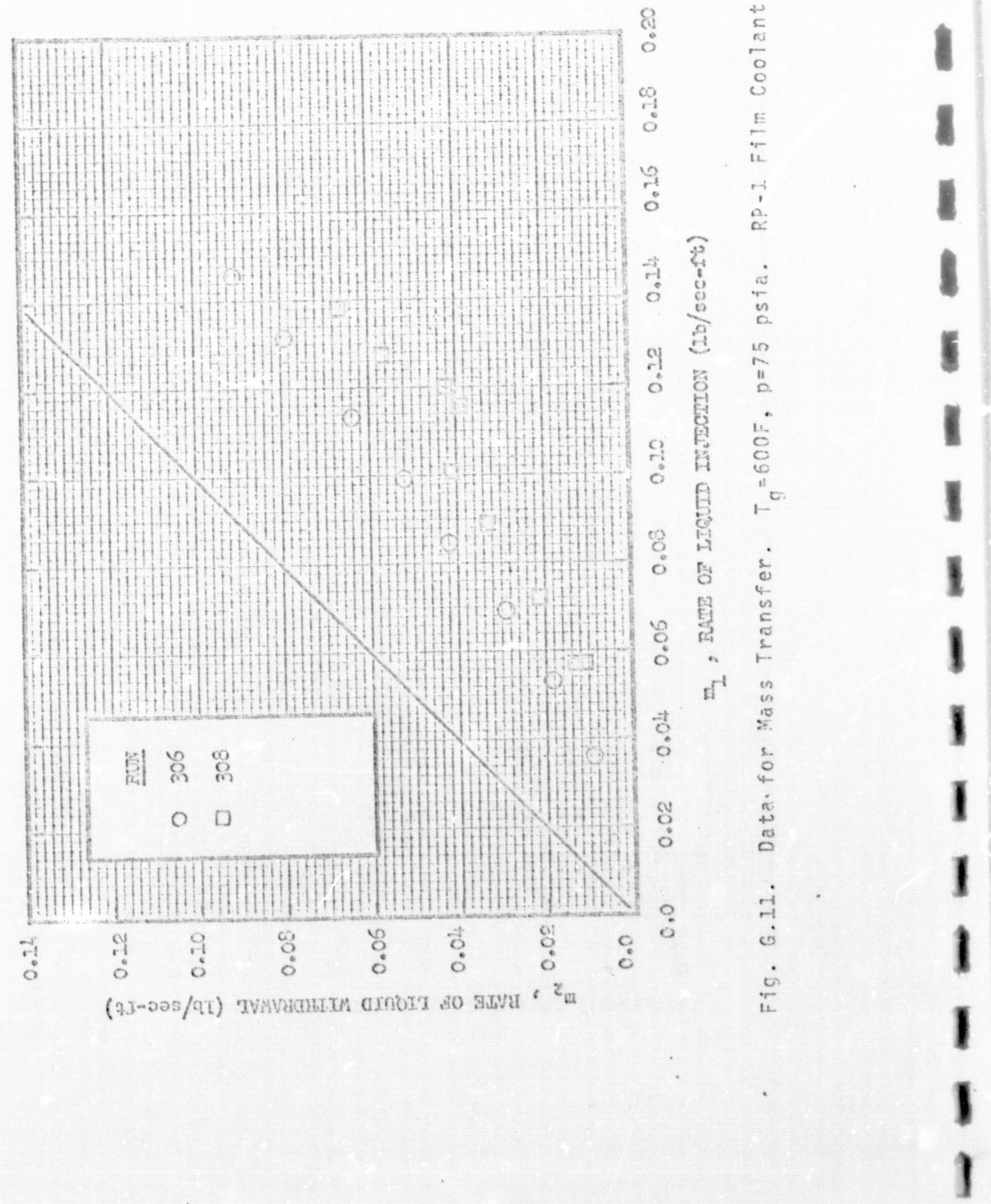




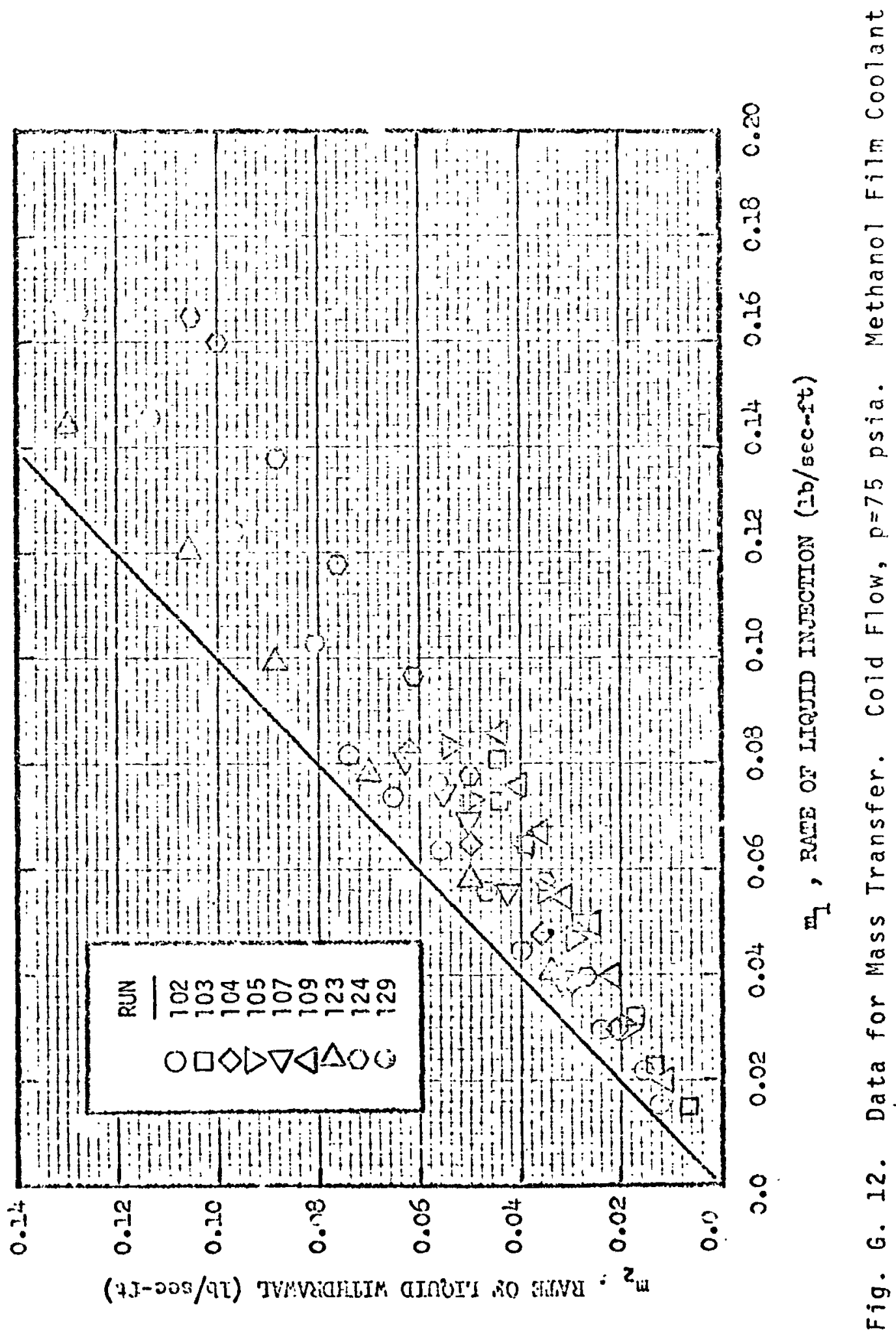



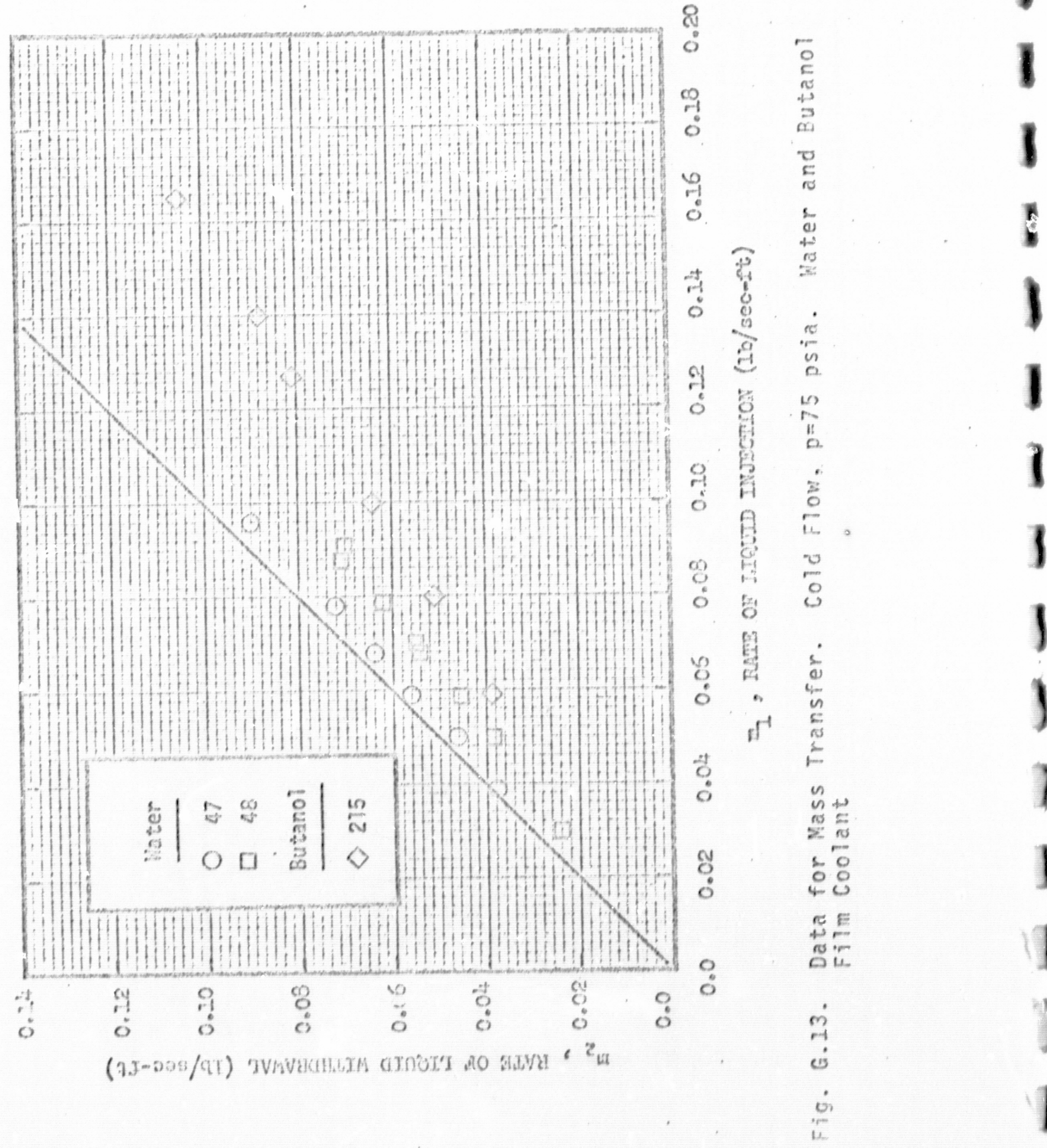

!
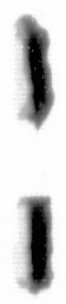

I

I

1

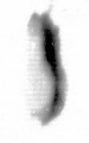

)

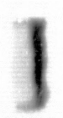

]

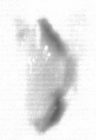

1

1 


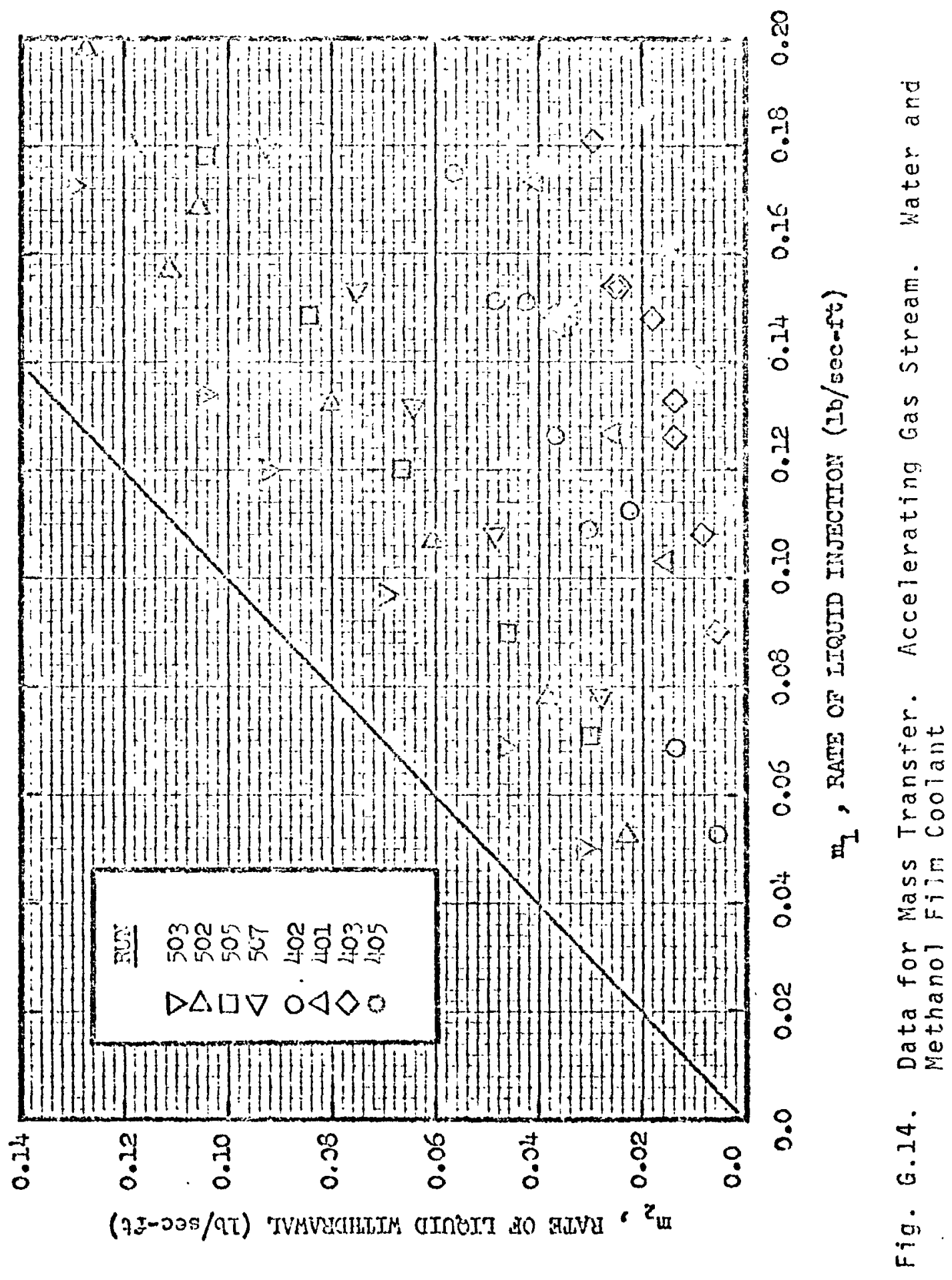

